\title{
Fluorescent reporters, calibration standards and endogenous protein labeling for quantitative single-molecule localization microscopy in cells
}

\author{
DISSERTATION \\ zur Erlangung des Doktorgrades \\ der Naturwissenschaften
}

vorgelegt beim Fachbereich Biochemie, Chemie und Pharmazie

der Johann Wolfgang Goethe-Universität

in Frankfurt am Main

Von

\section{Tim Niklas Baldering}

aus Bad Soden am Taunus

Frankfurt am Main (2020)

(D30) 

Vom Fachbereich Biochemie, Chemie und Pharmazie der Johann Wolfgang GoetheUniversität als Dissertation angenommen.

Dekan:

Gutachter:
Prof. Dr. Clemens Glaubitz

Prof. Dr. Mike Heilemann

Prof Dr. Gerhard Hummer

Datum der Disputation: 



\section{Zusammenfassung}

Die Kommunikation von Zellen mit ihrer Umgebung wird durch Rezeptorproteine arrangiert, die sich in der Plasmamembran befinden. Membranrezeptoren werden durch die Bindung von extrazellulären Liganden, Pathogenen oder Zell-Zell-Interaktionen aktiviert, wodurch die Bildung eines aktiven Zustands gefördert wird, der eine intrazelluläre Reaktion einleitet. Eine Beschreibung auf molekularer Ebene, wie sich Membranrezeptoren in Proteinanordnungen organisieren und wie diese Proteinanordnungen eine spezifische funktionelle Aufgabe ausführen, ist der Ausgangspunkt für das Verständnis der molekularen Mechanismen, die Gesundheit und Krankheit zugrunde liegen.

Die Fluoreszenzmikroskopie gibt Aufschluss über die Lage von Proteinen in Zellen, und mit der Einführung der höchstauflösenden Mikroskopie wurde der Nachweis einzelner Proteingruppierungen möglich. Eine Einschränkung der meisten Methoden der höchstauflösenden Mikroskopie ist, dass einzelne Komponenten einer Proteingruppierung optisch nicht aufgelöst werden können, was an der geringen Größe und dichten Packung der Bestandteile im Vergleich zur erreichbaren räumlichen Auflösung liegt. Eine Lösung, die für Einzelmolekül-Lokalisierungsmethoden gezeigt wurde, besteht darin, zusätzliche experimentelle Informationen in die Analyse zu implementieren, also „die Auflösungsgrenze der höchstauflösenden Mikroskopie zu umgehen". Bei der Einzelmolekül-Bildgebung kann diese zusätzliche Information zum Beispiel die Kinetik von mehrfachen und wiederkehrenden Emissionsereignissen sein, die bei einzelnen Fluorophoren beobachtet werden, was als "Blinken" bezeichnet wird. Diese Ereignisse können durch die Gesetze der Massenwirkung beschrieben werden, die von der Konzentration und damit der Anzahl der Moleküle abhängen, die zum Ensemble der Emissionsereignisse z. B. innerhalb einer Protein-Nanogruppierung beitragen. Weitere Parameter sind die charakteristischen Zeiten, die ein Fluorophor im „An“Zustand verweilt, der Kehrwert der Dissoziationsratenkonstante, und die Zeit zwischen zwei Emissionsereignissen, der Kehrwert der Assoziationsratenkonstante. Beide Konzepte können auf die beiden Klassen von Fluorophor-Markierungen angewendet werden, die in der Einzelmolekül-Lokalisationsmikroskopie (SMLM) verwendet werden, d.h. photoschaltbare Markierungen und transiente Markierungen. 
Das Ziel dieser Arbeit war die Entwicklung einer höchstauflösenden Fluoreszenzmikroskopiemethode zur Detektion von Proteinmonomeren und -dimeren in der Plasmamembran von Zellen. Als Reporter wurden photoschaltbare fluoreszierende Proteine verwendet, deren photoschaltbare Kinetik mit kinetischen Gleichungen analysiert wurde, die zuvor für die Angabe von Molekülzahlen gezeigt wurden. Synthetische, genetische und zelluläre Referenzproteine wurden konstruiert und dienten als Kalibrierungsreferenzen für monomere und dimere Proteine. Eine Methode zur stöchiometrischen endogenen Markierung von Proteinen wurde verwendet, um zwei Rezeptortyrosinkinasen, MET und EGFR, mit einem photoschaltbaren fluoreszierenden Protein zu markieren. Das Vorkommen von monomerem und dimerem MET-Rezeptor wurde auf der Plasmamembran von HEK293T-Zellen mittels quantitativer höchstauflösender Mikroskopie bestimmt. Der Diffusionskoeffizient und der Diffusionsmodus des MET-Rezeptors in lebenden HEK293T-Zellen wurden mit Einzelpartikelverfolgung gemessen.

Im ersten Teil dieser Arbeit wurde die Kinetik des photoschaltbaren Zyklus von einzelnen fluoreszierenden Proteinen analysiert. $\mathrm{Zu}$ diesem Zweck wurden die fluoreszierenden Proteine mEos3.2, mEos4b, mMaple3, Dendra2 und PAmCherry ausgewählt, die in der Einzelmolekül-Lokalisationsmikroskopie häufig eingesetzt werden. Diese Proteine wurden exprimiert, gereinigt, auf eine mit Poly-L-Lysin beschichtete Glasoberfläche aufgebracht und Einzelmolekül-Bildstapel aufgenommen. Die Kinetik des Photoschaltens und die Anzahl der Photonen pro Emissionsereignis wurden extrahiert. Die Proteine mEos3.2, mEos4b und mMaple3 zeigten optimale Helligkeits- und photoschaltbare Eigenschaften für die molekulare Quantifizierung mittels quantitativer photoaktivierter Lokalisationsmikroskopie (qPALM). Darüber hinaus wurde die Kinetik der Photoschaltung von mEos3.2, Dendra2 und mMaple3 durch ein Reduktionsmittel oder eine Punktmutation an der Aminosäure 69 manipuliert. Als nächstes wurden synthetische Dimere konstruiert, indem das fluoreszierende Protein mEos3.2 an doppelsträngige DNA angehängt wurde, die auf beiden Seiten mit einem Tris-NTAAnteil konjugiert war, wodurch ein Abstand von $\sim 10 \mathrm{~nm}$ entstand. Mit dem synthetischen Dimer konnte in vitro eine Detektionseffizienz von $78 \%$ für mEos3.2 ermittelt werden. Genetische Dimere wurden konstruiert, indem zwei fluoreszierende Proteine mit einem Peptidlinker von 21 Aminosäuren fusioniert wurden. Das genetische Dimer wurde 
exprimiert, gereinigt, an eine Poly-L-Lysin-Glasoberfläche angeheftet und Einzelmolekül-Bildstapel wurden aufgenommen. Die quantitative Analyse ergab eine Mischung aus $82 \%$ dimerem und $18 \%$ monomerem mEos3.2, was gut mit den $\sim 80 \%$ Dimeren übereinstimmt, die aus einem SDS-Gel bestimmt wurden. Als Kalibrierstandards für Experimente in Zellen wurden das monomere Plasmamembranprotein CD86 und das dimere Plasmamembranprotein CTLA-4 an mEos3.2 und mEos $4 \mathrm{~b}$ fusioniert und dienten als Kalibrierreferenzen in Zellen. Es wurden ähnliche Detektionseffizienzen von $76 \%$ für mEos3.2 in HeLa-Zellen und $79 \%$ für mEos4b in HEK293T-Zellen ermittelt.

Im zweiten Teil dieser Arbeit wurde das kinetische Modell, das zur Annäherung des Häufigkeitshistogramms von Blinkereignissen einzelner Fluorophore verwendet wird, auf Oligomere höherer Ordnung erweitert. Ein Vergleich mit einem zuvor entwickelten Modell zeigte, dass das erweiterte Modell genauere Ergebnisse für Oligomere höherer Ordnung und Mischungen verschiedener Oligomere liefert. Zusätzlich wird die Anwesenheit von unerkannten Oligomeren berücksichtigt. Das erweiterte Modell wurde an simulierten Daten von synthetischen Gruppierungen von photoschaltenden Fluorophoren mit unterschiedlicher Stöchiometrie getestet und gab die Grundwahrheit genau wieder. Studien, die das bisherige Modell verwendeten, wurden im Hinblick auf mögliche Abweichungen und deren Auswirkungen diskutiert. Da die Detektionseffizienz der fluoreszierenden Proteine hoch war und hauptsächlich Monomere und Dimere analysiert wurden, wurden nur geringe Abweichungen erwartet und gefunden. Die erweiterte Theorie bietet die Grundlage, um größere Oligomere und Mischungen unterschiedlicher Stöchiometrie mit besserer Genauigkeit zu untersuchen.

Im dritten Teil dieser Arbeit wurde eine Methode zur effizienten endogenen und stöchiometrischen Markierung von zellulären Proteinen mit photoschaltbaren fluoreszierenden Proteinen für die höchstauflösende Mikroskopie adaptiert. Aufbauend auf einem publizierten Protokoll wurde die CRISPR/Cas12a-unterstützte endogene Markierung von zwei Rezeptortyrosinkinasen, MET und EGFR, mit dem photoschaltbaren fluoreszierenden Protein mEos4b in HEK293T-Zellen erreicht. Der spezifische Einbau der DNA-Sequenz von mEos4b am C-Terminus des MET- und EGFR-Gens wurde durch Polymerase-Kettenreaktion und anschließende Sequenzierung der amplifizierten Produkte verifiziert. Die Markierungseffizienz wurde mittels Western- 
Blot-Analyse bestimmt und betrug $\sim 81 \%$ für MET und $\sim 25 \%$ für EGFR. Die Funktionalität der konstruierten Zelllinien wurde durch die Überwachung der nachgeschalteten Phosphorylierung der Mitogen-aktivierten Proteinkinase (MAPK) verifiziert.

Die effiziente Markierung des MET-Rezeptors mit mEos4b ermöglichte die molekulare Quantifizierung mit Einzelmolekül-Lokalisationsmikroskopie und zeigte den oligomeren Zustand von MET. In Abwesenheit von Liganden wurde fast ausschließlich monomeres MET an der Plasmamembran von HEK293T-Zellen gefunden ( 5\% Dimere), während der Anteil an dimerem MET auf ca. 71\% anstieg, wenn es mit dem physiologischen Liganden HGF und auf 63\% mit dem bakteriellen Liganden $\mathrm{InlB}_{321}$ behandelt wurde. Diese Ergebnisse deuten, in Übereinstimmung mit dem kanonischen Modell, auf eine ligandeninduzierte Bildung von MET-Dimeren hin.

Um die Dynamik des MET-Rezeptors in lebenden Zellen zu verfolgen, wurde eine Einzelpartikelverfolgung von MET-mEos4b durchgeführt. Die Einzelpartikelverfolgung erlaubt es, die Bewegung von individuellem MET-mEos4b an der Plasmamembran zu verfolgen und den Diffusionsmodus zu bestimmen, was auf Interaktionen des Rezeptors mit zellulären Komponenten hinweisen kann. Die individuellen Lokalisierungen von MET-mEos4b wurden zu Trajektorien verknüpft, aus denen die mittlere quadratische Verschiebung (mean square displacement, MSD) berechnet wurde. Die MSD liefert Informationen über die Diffusionsmodi, wie z. B. den Anteil der immobilen, eingeschlossenen und frei diffundierenden Rezeptoren. In Abwesenheit von Liganden wurden $15 \%$ der MET-mEos4b-Rezeptoren als immobil identifiziert, wohingegen der immobile Anteil in mit HGF behandelten Zellen auf $72 \%$ und in mit InlB $_{321}$ behandelten Zellen auf $27 \%$ anstieg. Dieser Teil der Arbeit zeigte, dass die Kombination von CRISPR/Cas12a-gestützter endogener Markierung und EinzelmolekülLokalisierungsmikroskopie ein leistungsfähiges Werkzeug zur Untersuchung der molekularen Organisation und Dynamik von Membranproteinen ist.

Im vierten Teil dieser Arbeit wurde die Einzelmoleküldatenanalyse durch ein Softwaretool beschleunigt, das eine automatisierte und unvoreingenommene Detektion von Einzelmolekül-Emissionsereignissen ermöglicht. Der Anteil von Monomeren und Dimeren von fluoreszierenden Reportern wurde durch die Implementierung eines neuronalen Netzwerks bestimmt (die Software wurde von Alon Saguy geschrieben; Gruppe von Prof. Yoav Shechtman, Technion, Israel). Der oligomere Zustand der IV 
monomeren und dimeren Referenzproteine CD86 und CTLA-4 wurde erfolgreich bestimmt. Die automatisierte Detektion einzelner Proteingruppierungen ermöglichte die Analyse von MET-mEos4b in einzelnen Zellen, wodurch die Heterogenität zwischen den Zellen bestimmt und das Expressionsniveau des Rezeptors mit der Dimerisierung korreliert werden konnte.

Zusammenfassend werden in dieser Arbeit Ergebnisse zu elementaren Aspekten hin zu einer molekularen Quantifizierung von Proteinzahlen mittels EinzelmolekülLokalisationsmikroskopie berichtet, die fluoreszierende Reporter, stöchiometrische Markierung von zellulären Proteinen und Bildanalyse umfassen. Das Potential dieser Entwicklungen wurde anhand der Beobachtung der Liganden-induzierten Verschiebung von monomeren zu dimeren MET-Rezeptoren in einzelnen HEK293T-Zellen gezeigt. 


\section{Summary}

Communication of cells with their environment is orchestrated through receptor proteins located in the plasma membrane. Membrane receptors are activated by binding extracellular ligands, pathogens or cell-cell interactions, promoting the formation of an active state that initiates an intracellular response. A molecular-level description of how membrane receptors organize into protein assemblies, and how these protein assemblies execute a specific functional task, is the entry point to understand molecular mechanisms that underlie health and disease.

Fluorescence microscopy reports on the location of proteins in cells, and with the advent of super-resolution microscopy, the detection of single protein clusters became possible. A limitation of most super-resolution microscopy methods is that individual components of a protein assembly cannot be resolved optically, which is because of the small size and dense packing of the constituents compared to the spatial resolution that can be achieved. One solution that was shown for single-molecule localization methods is to implement additional experimental information into the analysis, any by that, "bypass the resolution limit of super-resolution microscopy". In single-molecule imaging, this additional information can for example be the kinetics of multiple and recurring emission events observed for single fluorophores, which is termed "blinking". These events can be described by the laws of mass action, which depend on the concentration and thus the number of molecules that contribute to the ensemble of emission events e.g. within a protein nanocluster. Other parameters are the characteristic times that a fluorophore resides in the on-state, the inverse of the dissociation rate constant, and the time between two emission events, the inverse of the association rate constant. Both concepts can be applied to the two classes of fluorophore labels used in single-molecule localization microscopy (SMLM), i.e. photoswitchable labels and transient labels.

The aim of this thesis was to develop a super-resolution fluorescence microscopy method for the detection of protein monomers and dimers in the plasma membrane of cells. Photoswitchable fluorescent proteins were used as reporters, and their photoswitching kinetics were analyzed with kinetic equations that were previously shown to report molecular numbers. Synthetic, genetic and cellular reference proteins were constructed 
and served as calibration references for monomeric and dimeric proteins. A method for stoichiometric endogenous labeling of proteins was used to label two receptor tyrosine kinases, MET and EGFR, with a photoswitchable fluorescent protein. The occurrence of monomeric and dimeric MET receptor was determined on the plasma membrane of HEK293T cells using quantitative super-resolution microscopy. The diffusion coefficient and diffusion mode of MET receptor in live HEK293T cells were measured with singleparticle tracking.

In the first part of this thesis, the kinetics of the photoswitching cycle of single fluorescent proteins was analyzed. For this purpose, fluorescent proteins, mEos3.2, mEos4b, mMaple3, Dendra2, and PAmCherry, that are being prominently used in single-molecule localization microscopy, were selected. These proteins were expressed, purified, deposited on a glass surface coated with poly-L-lysine, and single-molecule image stacks were recorded. The kinetics of photoswitching and the number of photons per emission event were extracted. The proteins mEos3.2, mEos4b and mMaple3 showed optimal brightness and photoswitching properties for molecular quantification using quantitative photoactivated localization microscopy (qPALM). In addition, the kinetics of photoswitching of mEos3.2, Dendra2, and mMaple3 were manipulated by a reducing agent or a point mutation at amino acid 69. Next, synthetic dimers were constructed by appending the fluorescent protein mEos3.2 to double-stranded DNA conjugated with a tris-NTA moiety on both sides, generating a distance of $\sim 10 \mathrm{~nm}$. Using the synthetic dimer, a detection efficiency of $78 \%$ for $\mathrm{mEos} 3.2$ could be determined in vitro. Genetic dimers were constructed by fusing two fluorescent proteins with a peptide linker of 21 amino acids. The genetic dimer was expressed, purified, attached to a poly-L-lysine glass surface and single-molecule image stacks were recorded. Quantitative analysis revealed a mixture of $82 \%$ dimeric and $18 \%$ monomeric mEos3.2, which was well in line with $\sim 80 \%$ dimers determined from an SDS-gel. As calibration standards for experiments in cells, the monomeric plasma membrane protein CD86 and the dimeric plasma membrane protein CTLA-4 were fused to mEos3.2 and mEos $4 \mathrm{~b}$ and served as calibration references in cells. Similar detection efficiencies of $76 \%$ for mEos3.2 in HeLa cells and $79 \%$ for mEos4b in HEK293T cells were determined.

In the second part of this thesis, the kinetic model that is used to approximate the frequency histogram of blinking events of single fluorophores was extended to higher- 
ordered oligomers. A comparison to a previously developed model showed that the extended model provides more accurate results for higher-order oligomers and mixtures of different oligomers. In addition, the presence of undetected oligomers is considered. The extended model was tested on simulated data of synthetic clusters of photoswitching fluorophores with different stoichiometry and accurately reported the ground truth. Studies that used the previous model were discussed in terms of possible deviations and their impacts. Since the detection efficiency of the fluorescent proteins was high and primarily monomers and dimers were analyzed, only minor deviations were expected and found. The extended theory provides the foundation to study larger oligomers and mixtures of different stoichiometry with better accuracy.

In the third part of this thesis, a method for efficient endogenous and stoichiometric labeling of cellular proteins with photoswitchable fluorescent proteins was adapted for super-resolution microscopy. Building on a published protocol, CRISPR/Cas 12a-assisted endogenous labeling of two receptor tyrosine kinases, MET and EGFR, with the photoswitchable fluorescent protein mEos4b was achieved in HEK293T cells. The specific incorporation of the DNA sequence of mEos $4 b$ at the C-terminus of the MET and EGFR gene was verified by polymerase chain reaction and subsequent sequencing of the amplified products. The labeling efficiency was determined by western blot analysis and found to be $\sim 81 \%$ for MET and $\sim 25 \%$ for EGFR. The functionality of the constructed cell lines was verified by monitoring the downstream phosphorylation of the mitogen activated protein kinase (MAPK).

The efficient labeling of the MET receptor with mEos $4 b$ enabled molecular quantification with single-molecule localization microscopy and reported the oligomeric state of MET. In the absence of ligands, almost exclusively monomeric MET was found at the plasma membrane of HEK293T cells ( $\sim 5 \%$ dimers), while the fraction of dimeric MET increased to approximately $71 \%$ when treated with the physiological ligand HGF and to $63 \%$ with the bacterial ligand $\mathrm{InlB}_{321}$. These results hint at a ligand-induced formation of MET dimers, in line with the canonical model.

Single-particle tracking of MET-mEos4b was performed to monitor the dynamics of the MET receptor in living cells. Single-particle-tracking allows following the motion of individual MET-mEos $4 \mathrm{~b}$ at the plasma membrane and determining the diffusion mode, which may hint at interactions of the receptor with cellular components. The individual localizations of MET-mEos $4 b$ were linked to trajectories from which the mean-square VIII 
displacement (MSD) was calculated. The MSD provides information on diffusion modes, such as the fraction of immobile, confined and free diffusing receptors. In the absence of ligand, $15 \%$ of the MET-mEos $4 \mathrm{~b}$ receptors were identified as immobile, whereas the immobile fraction increased to $72 \%$ in cells treated with HGF and to $27 \%$ in cells treated with InlB ${ }_{321}$. This part of the thesis demonstrated that the combination of CRISPR/Cas12a-assisted endogenous labeling and single-molecule localization microscopy is a powerful tool to study the molecular organization and dynamics of membrane proteins.

In the fourth part of this thesis, single-molecule data analysis was accelerated by a software tool that enables automated and unbiased detection of single-molecule emission events. The proportion of monomers and dimers of fluorescent reporters was determined by implementing a neural network (the software was written by Alon Saguy, group of Prof. Yoav Shechtman, Technion, Israel). The oligomeric state of the monomeric and dimeric reference proteins CD86 and CTLA-4 was successfully determined. The automated detection of single protein clusters allowed the analysis of MET-mEos $4 \mathrm{~b}$ in single cells, providing access to determine the heterogeneity between cells, and to correlate receptor expression level to dimerization.

In summary, this thesis reports results on elementary aspects towards molecular quantification of protein numbers using single-molecule localization microscopy, covering fluorescent reporters, stoichiometric labeling of cellular proteins and image analysis. The potential of these developments was shown by monitoring the ligandinduced shift from monomeric to dimeric MET receptors in single HEK293T cells. 


\section{Contents}

Zusammenfassung

Summary

1 Introduction 1

$\begin{array}{ll}\text { 1.1 Fluorescence microscopy in cell biology } & 1\end{array}$

1.1.1 Quantitative super-resolution microscopy 4

1.1.2 Calibration standards in super-resolution microscopy 6

$\begin{array}{ll}1.2 \text { Receptor tyrosine kinases } & 7\end{array}$

$\begin{array}{ll}\text { 1.2.1 MET receptor } & 10\end{array}$

$\begin{array}{ll}1.3 \text { Aims of this study } & 11\end{array}$

2 Theory $\quad 12$

2.1 Absorption and Fluorescence $\quad 12$

$\begin{array}{ll}2.2 \text { Fluorescent probes } & 14\end{array}$

2.2.1 Organic fluorophores 15

2.2.2 Fluorescent proteins 15

2.3 Fluorophore labeling strategies $\quad 18$

2.3.1 CRISPR/Cas System 21

2.3.2 CRISPR/Cas12a-assisted genome labeling 22

2.4 Fluorescence microscopy 23

2.4.1 Illumination schemes in fluorescence microscopy 24

2.4.2 Diffraction limit 25

2.5 Super-resolution microscopy 26

2.5.1 Single-molecule localization microscopy 26

2.5.2 Photoactivated localization microscopy 29

$\begin{array}{ll}2.6 \text { Quantitative SMLM } & 30\end{array}$

2.7 Single-particle tracking $\quad 32$

3 Methods $\quad \mathbf{3 5}$

3.1 Generation of plasmids $\quad 35$

$\begin{array}{ll}3.2 \text { Electroporation } & 37\end{array}$

3.3 Protein expression and purification in E.coli 37

3.4 Generation of a 30-base pair DNA-linker for dimerization of
His-tagged mEos3.2

$\begin{array}{ll}3.5 \text { Cell lines } & 38\end{array}$

3.6 Generation of PCR cassettes for CRISPR/Cas12a assisted
PCR tagging

3.7 Transfection $\quad 39$

3.8 Generation of stable cell lines $\quad 39$

3.9 Isolation and analysis of genomic DNA $\quad 40$ 
3.10 Western blot 40

3.11 Preparation of functionalized glass slides for SMLM 41

3.12 Fluorescence microscopes 41

3.13 Sample preparation for qPALM measurements in vitro 43

3.14 Sample preparation for qPALM measurements in cellulo 43

3.15 qPALM imaging $\quad 43$

3.16 qPALM analysis $\quad 44$

3.17 Sample preparation for sptPALM and uPAINT measurements $\quad 44$

3.18 sptPALM and uPAINT imaging $\quad 44$

3.19 sptPALM and UPAINT analysis $\quad 45$

3.20 Stochastic simulation of photophysics tool (ssp) 45

4 Results \& Discussion $\quad \mathbf{4 7}$

4.1 Calibration references for quantitative PALM 48

4.1.1 Blinking characteristics of photoactivatable and -convertible fluorescent proteins $\quad 48$

4.1.2 Synthetic and genetic dimers as quantification rulers for qPALM experiments 53

4.1.3 In cellulo calibration rulers for the determination of qPALM parameters $\quad 54$

4.1.4 Simulations of higher-ordered oligomers and mixtures of monomers and dimers $\quad 56$

$\begin{array}{ll}\text { 4.1.5 Discussion } & 57\end{array}$

4.2 Extending the theory of qPALM 63

4.2.1 A comparison of two models that describe simulated qPALM data 64

4.2.2 Relation between the q-value (model 1) and the detection efficiency (model 2) 65

4.2.3 Consideration of undetected oligomers in qPALM $\begin{array}{ll}\text { experiments } & 67\end{array}$

4.2.4 Accurate determination of mixtures of higher oligomers $\quad 69$

4.2.5 Simulated qPALM data of oligomers using mEos3.2 $\begin{array}{ll}\text { blinking statistics } & 70\end{array}$

$\begin{array}{ll}\text { 4.2.6 Discussion } & 72\end{array}$

4.3 CRISPR/Cas12a-assisted endogenous tagging of membrane receptors for super-resolution microscopy 74

4.3.1 Generation of stable cell lines via CRISPR/Cas12aassisted genome editing (PCR tagging) 75

4.3.2 Analysis of the oligomerization state of the MET receptor 81

4.3.3 Analysis of the dynamics of the MET receptor 84

4.3.4 Discussion 86 
4.4 Unbiased, automated analysis of single-molecule localization $\begin{array}{ll}\text { microscopy data } & 91\end{array}$

4.4.1 Automated detection of single-molecule emission events 91

4.4.2 Automated, unbiased quantification of protein monomers and dimers in cells $\quad 92$

4.4.3 Discussion 93

5 Conclusion \& Outlook $\quad 95$

5.1 Calibration references for quantitative PALM 95

5.2 Extending the theory of qPALM 96

5.3 CRISPR/Cas12a-assisted endogenous protein labeling for super-resolution microscopy $\quad 97$

5.4 Quantification with QAFKA surpasses manual analysis $\quad 98$

$\begin{array}{lr}6 \text { References } & 100\end{array}$

$\begin{array}{lr}\text { Appendix } & 119\end{array}$

$\begin{array}{ll}\text { List of abbreviations } & 121\end{array}$

$\begin{array}{lr}\text { List of figures } & 123\end{array}$

$\begin{array}{ll}\text { List of tables } & 125\end{array}$

$\begin{array}{lr}\text { Acknowledgements } & 126\end{array}$

$\begin{array}{lr}\text { Curriculum Vitae } & 128\end{array}$

$\begin{array}{lr}\text { Publications } & 130\end{array}$ 


\section{Introduction}

The molecular assembly of proteins into functional units determines their cellular function. Different types of homomers (oligomers) are associated with different biological functions according to their symmetry and probably closely related to allosteric regulation (Bergendahl und Marsh 2017). The knowledge about the formation of different oligomers can serve the development of pharmaceutical therapies, since diseases are often associated with dysfunctions and therefore disease-related protein associations can be specifically modulated (Liu et al. 2015).

Membrane proteins, such as receptor tyrosine kinases, regulate the communication of cells with their environment and play a crucial role in various diseases (Lemmon und Schlessinger 2010). The interaction between these receptors usually occurs by transient rather than covalent binding, which is why analysis in a native environment is most appropriate. Fluorescence microscopy is particularly well suited to analyze the localization, organization and dynamics of proteins directly in cells, as the method benefits from the high sensitivity and specificity of fluorescence (Shashkova und Leake 2017). In recent decades, fluorescence microscopy has greatly improved its spatial resolution through the development of super-resolution techniques and approaches a true "molecular resolution" of about $1 \mathrm{~nm}$ in cells (Balzarotti et al. 2017).

In this thesis, three main topics were addressed: (i) a method to read-out the stoichiometry of protein assemblies by analyzing single-molecule kinetic data in super-resolution microscopy was developed; (ii) quantitative genomic fluorescence labeling and its application in super-resolution microscopy was demonstrated; (iii) the molecular assembly and dynamics of the receptor tyrosine kinase MET in single cells was investigated.

\subsection{Fluorescence microscopy in cell biology}

Various methods exist that can report on protein numbers and interactions in complexes, such as immunoprecipitation (Kaboord und Perr 2008), mass spectrometry (Olshina und Sharon 2016), NMR spectroscopy (Marion 2013) or fluorescence microscopy (Shashkova und Leake 2017). However, most of these methods require cell disruption 
and purification of the proteins of interest, which makes the analysis of protein interactions in the cellular environment challenging. Due to their high sensitivity and specificity, fluorescence techniques are particularly suitable for the analysis of protein numbers and interactions directly in cells (Shashkova und Leake 2017).

The first fluorescence microscope was constructed at the beginning of the 20th century by the companies Carl Zeiss and Carl Reichert (Renz 2013). The development of fluorescently labeled antibodies (Coons et al. 1941) and the discovery of the green fluorescent protein (GFP) (Prasher et al. 1992) enabled the targeted labeling and visualization of cellular structures. In the early 1990s, different techniques were suggested to observe single molecules, which turned optical detection and fluorescence spectroscopy on a new level (Moerner et al. 2015). Additionally, tools that aim to determine molecular dynamics and interactions were developed. Fluorescence recovery after photobleaching (FRAP) analyzes molecules in living cells and extracts ensemble dynamics on a timescale of seconds to minutes (Rayan et al. 2010) (Figure 1.1 A). In contrast, fluorescence correlation spectroscopy observes only a few molecules in a tiny volume on a millisecond timescale (Hess et al. 2002) (Figure 1.1 B). Single-particle tracking allows the observation of single molecule trajectories by connecting single localizations in time (Manzo und Garcia-Parajo 2015) (Figure 1.1 C). The dynamic information obtained from these methods allows the assignment of different biological states. In addition, interactions of biomolecules can be suggested. Förster-resonance energy transfer is used to study molecular interactions that rely in the range of 1-10 nm (Padilla-Parra und Tramier 2012) (Figure 1.1 D). In FRET, the energy of a donor molecule is transferred to an acceptor molecule. By exciting donor molecules and using the acceptor fluorescence as read-out, information on molecular interactions between donor and acceptor molecules can be indicated. Another important finding that can provide insight into biological function of target proteins is their stoichiometry in protein complexes. A frequently used quantitative tool are photobleaching experiments, which provide information about protein numbers in oligomers (Gordon et al. 2004) (Figure 1.1 E). This technique is based on counting the number of bleaching steps in diffractionlimited intensity-time traces and is limited to low density samples. At higher densities, super-resolution techniques are required. 
A Fluorescence recovery after photobleaching (FRAP)
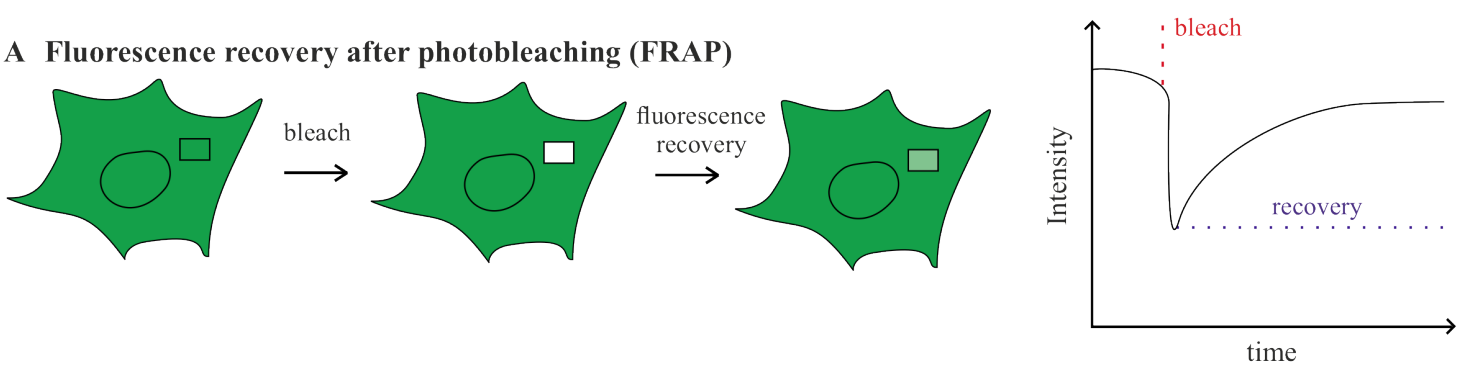

B Fluorescence correlation spectroscopy (FCS)
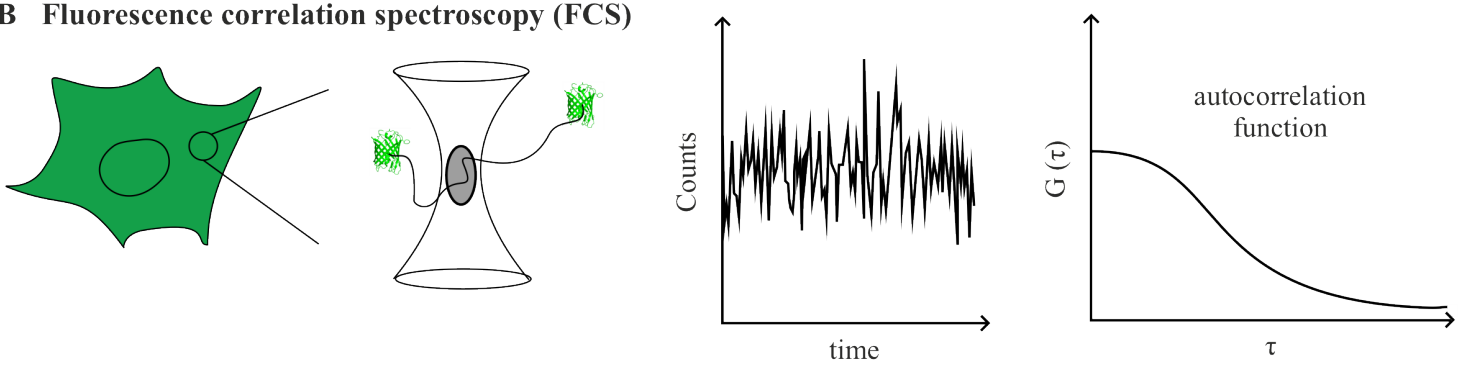

C Single-particle tracking (SPT)
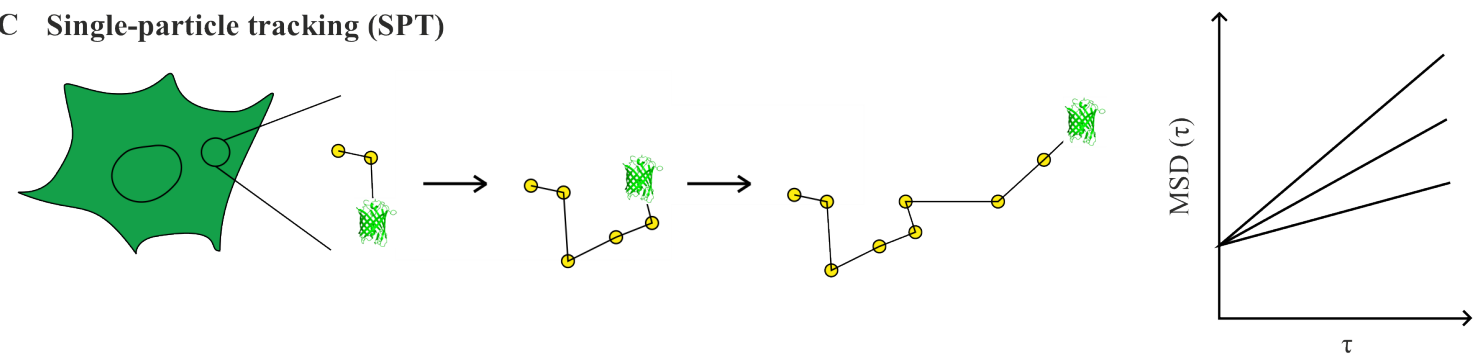

D Fluorescence resonance energy transfer (FRET)

E Photobleaching experiments
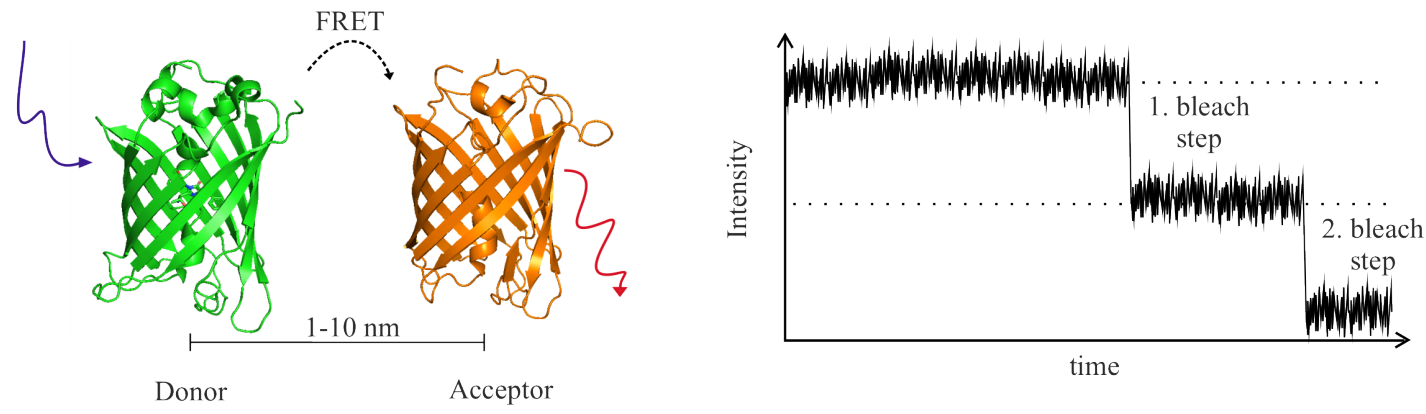

Figure 1.1: Fluorescence microscopy tools to assess the dynamics and interactions of proteins. (A) Fluorescence recovery after photobleaching (FRAP): A distinct area of the cell is photobleached which decreases the fluorescence intensity. Due to diffusion, the fluorescence is recovered. The time of recovery correlates with the diffusion coefficient of the ensemble. (B) Fluorescence correlation spectroscopy (FCS): A tiny volume is observed in which fluctuations of fluorescence signals are measured. From these fluctuations, an autocorrelation function is generated describing the number of particles and the diffusion coefficient. (C) Single-particle tracking (SPT): Single molecules are localized in time and the localizations are connected to trajectories. The mean square displacements (MSD) for different time lags (tau) are calculated and the slope is proportional to the diffusion coefficient. (D) Förster resonance energy transfer (FRET): The donor molecule is excited and transfers the energy to the acceptor molecule. The acceptor is excited and emits photons of a red-shifted wavelength. (E) Photobleaching 


\section{Introduction}

experiments: The intensity of a diffraction-limited spot is observed over the time. The number of photobleaching steps is proportional to protein numbers.

\subsubsection{Quantitative super-resolution microscopy}

At the beginning of the 21 st century, microscopy techniques that bypass the diffraction limit of light and enable nearly molecular resolution were developed. Single-molecule localization microscopy (SMLM) is one super-resolution microscopy technique that employs spatiotemporal separation of individual fluorescence emitters by stochastic activation, light-induced switching of fluorescence or transient binding of fluorophore labels. Hence, spatially separated single molecules are detected and the localization can be determined by approximating the signals with Gaussian functions. However, a single molecule can show recurrence of fluorescence, termed blinking, which complicates the quantification of target proteins. To overcome this overestimation, different approaches such as (i) averaging the number of localizations and comparing them with calibrations, (ii) correlation analysis of single-molecule emission events, (iii) photophysical models describing the kinetics of fluorescence blinking, and (iv) methods for analyzing the binding kinetics of DNA hybridization have been developed (Figure 1.2) (Dietz und Heilemann 2019). 

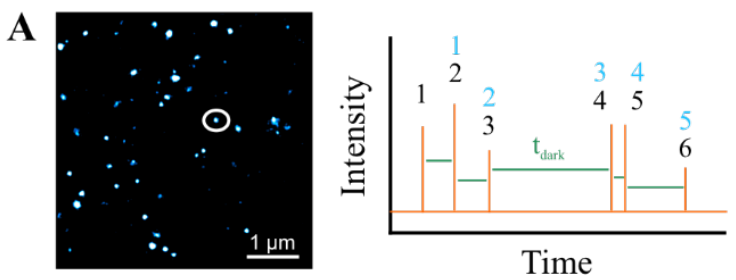

C

Pair-correlation
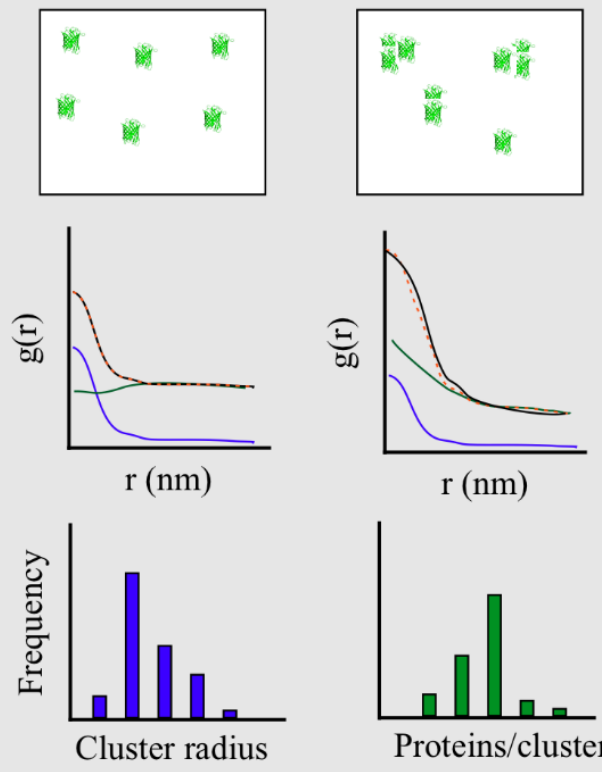

$\mathrm{r}(\mathrm{nm})$

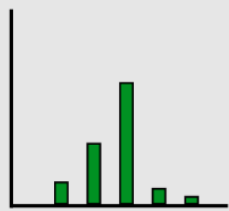

Proteins/cluster

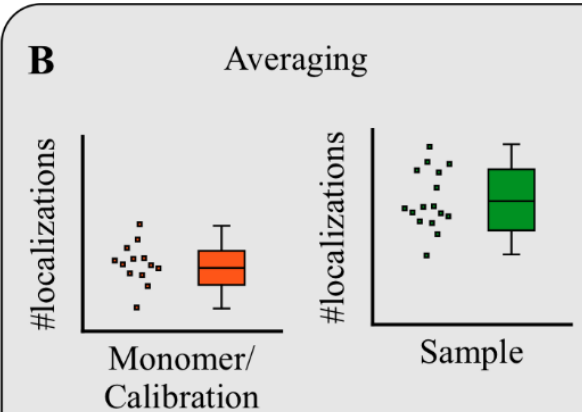

D

qPALM
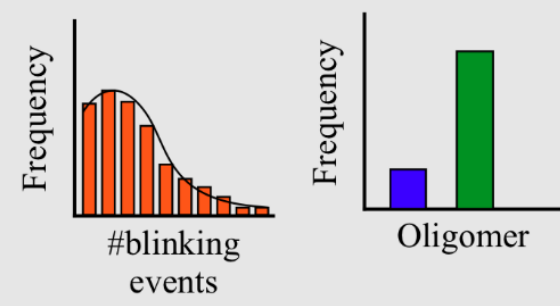

$\mathbf{E}$

qPAINT

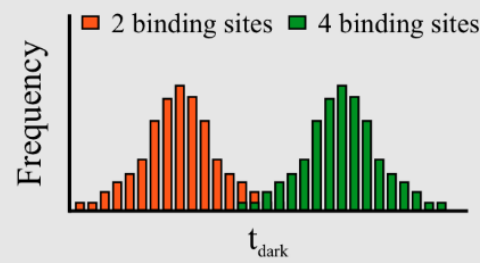

Figure 1.2: Quantification in single-molecule localization microscopy. (A) PALM image showing localizations emerging from one or more proteins (left) and an exemplary intensity time trace of a localization spot (white circle) (right). The number of fluorescence events (black, B), the number of blinking events (light blue, D); and the dark time (green, $\left.t_{\text {dark }}\right)(E)$ are determined. (B) The number of localizations is determined for a single target protein and is compared to the respective sample. (C) Pair-correlation analysis determines the spatial correlation of fluorescence particles by a pair-correlation function and the cluster radii as well as the number of proteins per cluster are determined. (D) qPALM counts the number of blinking events per localization cluster and a blinking distribution is obtained. This distribution is approximated by functions describing the photophysics of oligomers. (E) qPAINT analyzes the time between successive emission events $\left(t_{\text {dark }}\right) \cdot t_{\text {dark }}$ is anti-proportional to the number of proteins in the localization cluster, and its distribution is compared to dark times of calibration molecules.

Quantitative microscopy requires stoichiometric and homogenous labeling of proteins with fluorophore labels. A defined stoichiometry is achieved by genetically encoding fluorescent proteins to the target protein. By transfection of plasmids, the fusion proteins are expressed in the cell. However, the number of plasmids transfected in cells can 
typically vary widely, resulting in different and unnatural expression levels of the target protein. Thanks to advances in genome editing, CRISPR/Cas technology allows fast and specific labeling of endogenous proteins with fluorescent proteins (Ran et al. 2013). This approach is very well suited for quantification as it provides a homogenous and native expression with a defined one-to-one stoichiometry. Another important parameter for quantification is the detection efficiency. Incomplete chromophore maturation or misfolded fluorescent proteins can lead to an underestimation of protein numbers. This underestimation can be compensated by including a detection efficiency derived from reference proteins that form defined protein numbers in complexes.

\subsubsection{Calibration standards for super-resolution microscopy}

Model proteins with a well-defined stoichiometry may also serve as reference in quantitative microscopy. Such reference proteins require evidence that confirms their existence as the respective oligomer. For example, bacterial homo-oligomers such as FsaA, GlnA, Dps, and FtnA have been structurally characterized by X-ray crystallography and used as calibration standards for fluorescence microscopy (Finan et al. 2015). In eukaryotic cells, the nuclear pore complex was demonstrated to be useful to serve as calibration standard for quantification (Thevathasan et al. 2019). The generation of stable cell lines of Nup96 tagged with different fluorescent proteins or affinity tags allowed to quantify labeling efficiencies and serve as precise reference for molecular counting. Photoactivation efficiencies of various fluorescent proteins were also determined by expressing the human glycine receptor in Xenopus oocytes and analyzing the number of photobleaching steps (Durisic et al. 2014).

Important for this thesis are the monomeric cluster of differentiation 86 (CD86) and homodimeric cytotoxic T-lymphocyte-associated protein 4 (CTLA-4). These two reference proteins are membrane proteins that are involved in the regulation of T-cell activation and are members of the immunoglobulin (Ig) superfamily. Different methods such as fluorescence recovery after photobleaching, resonance energy transfer, crystallography, and analytical ultracentrifugation verified the monomeric existence of CD86 (Collins et al. 2002; Zhang et al. 2003; Bhatia et al. 2005; James et al. 2006; Dorsch et al. 2009; Girard et al. 2014). Two CTLA-4 proteins form a homodimer via disulfide bonds and bind to CD86 (Slavik et al. 1999). The existence as a homodimer has been indicated in resonance energy transfer and various biochemical studies (Linsley et al. 1995; Greene et al. 1996; Bhatia et al. 2005; Darlington et al. 2005; James et al. 2006). 
Once the properties of these reference proteins have been investigated, investigations can be performed on unknown samples to confirm their existence as monomer or dimer. However, these investigations are not limited to pure monomers or dimers, but also to mixtures of both oligomer types. For instance, membrane proteins such as receptor tyrosine kinases exist both as monomer and as dimer.

\subsection{Receptor tyrosine kinases}

Receptor tyrosine kinases (RTKs) are cell-surface receptors that serve as key regulators of critical cellular processes, such as cell survival, cell metabolism, cell migration, cell cycle, proliferation and differentiation (Lemmon und Schlessinger 2010). These cellular processes are triggered by different growth factors that bind their respective receptors.

The structure and key regulatory role of RTKs is highly conserved between different organisms. In humans, 58 different RTKs exist, that are categorized into 20 subfamilies. Among these a common structure is preserved that is directly related to their signaling function (Hubbard und Till 2000). The molecular architecture comprises a ligand binding domain in the extracellular region, a single hydrophobic transmembrane helix, and a cytoplasmic region that contains the tyrosine kinase domain and juxtamembrane regulatory regions (Figure 1.3) (Lemmon und Schlessinger 2010).

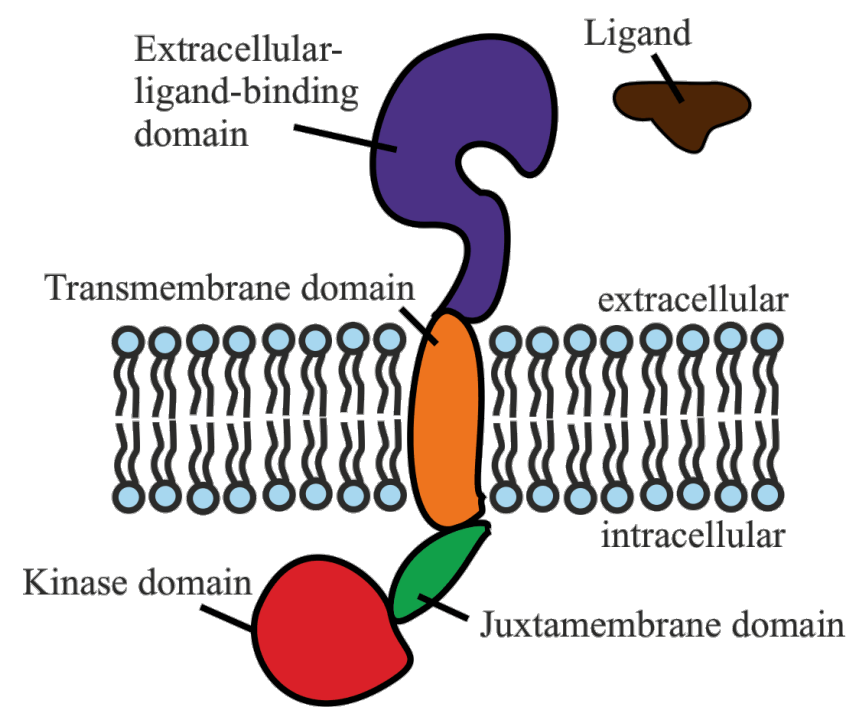

Figure 1.3 Structure of receptor tyrosine kinases. Receptor tyrosine kinases consist of an extracellular ligand-binding domain (blue), a transmembrane domain (orange), a juxtamembrane domain (green) and a kinase domain (red). For activation of RTKs, a ligand (brown) binds to the extracellular domain. 


\section{Introduction}

Receptor activation is initiated by binding of specific ligands, which commonly induces conformational changes in the receptor that enable dimerization and interaction of the tyrosine kinase domain with juxtamembrane regulatory regions (Ullrich und Schlessinger 1990). Although ligand-induced dimerization may be the general case, previous studies also showed that a subset of RTKs form dimers even in the absence of ligands (Clayton et al. 2005; Ward et al. 2007; Dietz et al. 2013). However, in addition to dimerization, ligand-bound receptors leave the auto-inhibitory conformation so that key tyrosine residues are phosphorylated. The phosphorylated tyrosines serve as docking sites for intracellular adaptor and signaling proteins (Ullrich und Schlessinger 1990; Lemmon und Schlessinger 2010). Signaling proteins are mostly multivalent (Li et al. 2012), resulting in a protein assembly that amplifies the signal of individual receptor clusters. This initiates a cascade of signaling processes that result in transcriptional regulation of distinct genes and enables the cell to adapt to the microenvironment (Schlessinger 2000). Downregulation of signaling occurs via endocytosis of receptors (Dikic 2006) and finally their ubiquitinoylation and degradation in lysosomes (Critchley et al. 2018).

Abnormal RTK activity in human cancers is mediated for example by autocrine activation, chromosomal translocations, RTK overexpression or gain-of-function mutations (Lemmon und Schlessinger 2010). This connection of RTKs to different types of cancer such as lung cancer (Hynes und Schlange 2006; Sharma et al. 2007) underlines the importance of RTK research for pharmaceutical therapy (Blume-Jensen und Hunter 2001; Krause und van Etten 2005; Hubbard und Miller 2007). Mutations that cause genetic changes or abnormalities are not only associated with cancer, but also with diabetes, inflammation, severe bone disorders, arteriosclerosis, and angiogenesis (Lemmon und Schlessinger 2010). Research on RTKs and their mutations is therefore of great importance, and massive progress in understanding their structure and mechanisms involved in RTK activity was achieved (Lemmon und Schlessinger 2010).

The epidermal growth factor receptor (EGFR), also known as ErbB1/HER1, is the best studied RTK and belongs to the ErbB/HER family. The ErbB/HER family includes not only EGFR but also ErbB2, ErbB3, and ErbB4. These family members can form different combinations of homo- or heterodimers (Muthuswamy et al. 1999), leading to the activation of several signaling pathways such as the mechanistic Target of Rapamycin (mToR), mitogen activated protein kinase (MAPK), p21-activated kinase (PAK), and signal transducer and activator of transcription (STAT) pathway (Figure 1.4). These pathways initiate a variety of physiological and pathological responses, including growth, 
migration, proliferation, differentiation, and inhibition of apoptosis (Lewis et al. 1998; Morrison 2012).

The EGFR gene is a protooncogene located on the short arm q22 of chromosome 7 that is transcribed and translated into a 1210 residue precursor protein that, after cleavage at the N-terminus, leads to a mature EGFR containing 1186 amino acids. The extracellular ligand-binding domain of EGFR, consisting of leucine-rich and cysteine-rich domains, is essential for the interaction with the $6 \mathrm{kDa}$ natural ligand epidermal growth factor (EGF). The interaction of EGF with EGFR initiates receptor dimerization and transphosphorylation of tyrosine residues located at the C-terminus of EGFR. Signaling of EGFR is regulated by endocytosis via clathrin-coated pits (Roepstorff et al. 2008; Madshus und Stang 2009), ubiquitination and degradation in lysosomes (Citri und Yarden 2006).

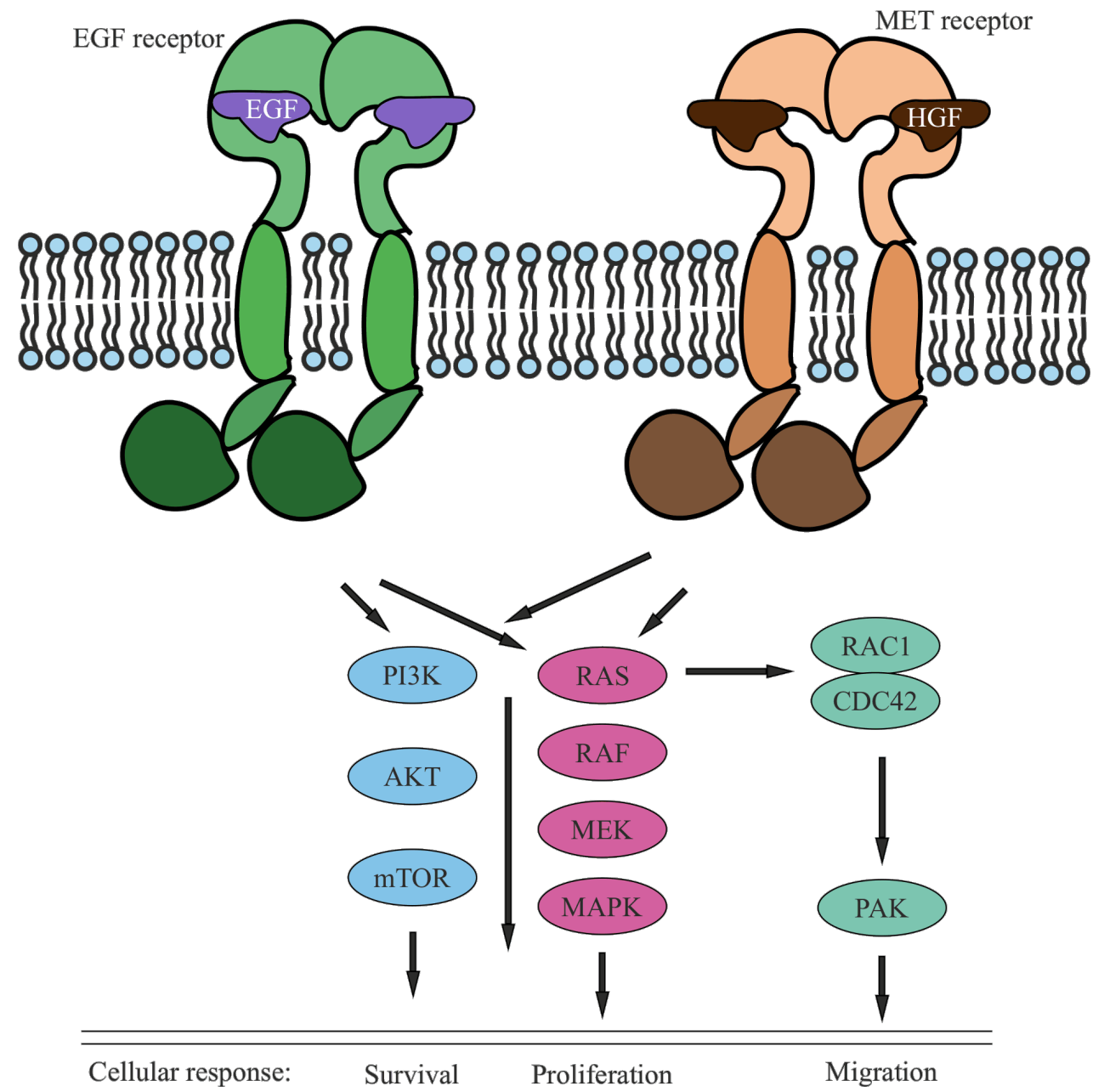

Figure 1.4: Overview of MET and EGFR signaling pathways. Schematic illustration of EGFR (green) and the MET receptor (beige-brown) at the cell membrane bound to their native ligands, EGF (purple) and HGF (brown), respectively. Ligand binding activates several pathways such as 
mTOR (light blue), MAPK (pink), and PAK (turquoise) leading to cell survival, proliferation, and migration.

\subsubsection{MET receptor}

The MET receptor, also known as hepatocyte growth factor receptor (HGFR), is an RTK that is expressed on the cell membrane of various epithelial cells in many organs including liver, pancreas, prostate, muscle, and kidney (Furge et al. 2000; Comoglio et al. 2018; Zhang et al. 2018). The MET receptor is involved in embryogenesis (Bladt et al. 1995; Schmidt et al. 1995; Uehara et al. 1995) and wound healing in vertebrates (Nakamura et al. 2000; Matsumoto und Nakamura 2001). The MET gene is an protooncogene located on chromosome 7q21-31 (Cooper et al. 1984; Organ und Tsao 2011) and encodes a protein with 1390 amino acids (Baldanzi und Graziani 2014). The MET receptor is formed by proteolytic processing of a precursor to a heterodimer consisting of an alpha $(50 \mathrm{kDa})$ and a beta chain $(145 \mathrm{kDa})$ linked by disulfide bonds (Trusolino und Comoglio 2002). The mature protein comprises an N-terminal semaphorin (sema) domain, a plexin, semaphorin, and integrin (PSI) domain, and an immunoglobulin-like Ig-like domain in the extracellular region, followed by the transmembrane domain, a juxtamembrane domain (containing Y1003), a kinase domain (containing Y1234/5) and a multifunctional docking site (containing Y1349/56) (Trusolino und Comoglio 2002; Birchmeier et al. 2003; Organ und Tsao 2011).

Analog to other RTKs, MET serves as an interface between external ligands and intracellular signal transduction. In the resting state, MET is assumed to exist predominantly as monomer with a small fraction of pre-assembled dimers (Dietz et al. 2013; Li et al. 2020). Upon binding of the native ligand of MET, the hepatocyte growth factor (HGF), the receptor dimerizes and is activated. The signal cascade is initiated by transphosphorylation of the tyrosine residues Y1349 and Y1356 of MET receptors by their kinase domains. These phosphorylated tyrosine residues serve as docking sites for intracellular adaptor and signaling proteins such as growth factor receptor-bound protein 2 (Grb2), Grb2-associated-binding protein 1 (Gab1) or phosphoinositide 3-kinase (PI3K) (Ponzetto et al. 1994; Weidner et al. 1996; Furge et al. 2000; Birchmeier et al. 2003), which leads to the activation of common signaling pathways such as mToR, MAPK, and PAK pathways (Figure 1.4) (Birchmeier et al. 2003). These signaling pathways promote cellular motility, cell differentiation, proliferation, cell adhesion, cytoskeletal rearrangement, and cell survival (Furge et al. 2000; Birchmeier et al. 2003). 
Ubiquitination of MET at tyrosine Y1003 leads to endocytosis and degradation of the receptor in lysosomes (Hammond et al. 2001).

Despite that, signaling of MET can be initiated by binding of internalin B (InlB) of the pathogenic bacterium Listeria monocytogenes (Braun et al. 1998). Listeria monocytogenes causes listeriosis in humans by using internalin A and B to invade different types of host cells (Braun et al. 1998). The soluble form of InlB binds MET in a 2:2 stoichiometry (Ferraris et al. 2010) and has been shown to increase dimerization of MET (Dietz et al. 2013).

\subsection{Aims of this study}

The main objectives of this study were (i) the generation of monomeric and dimeric reference systems for quantification, (ii) the advancement of quantitative single-molecule localization microscopy, and (iii) the establishment of endogenous stoichiometric labeling.

The first aim was to investigate the photophysical kinetics of five different photoactivatable and -convertible fluorescent proteins, and to explore how kinetic information can serve for molecular quantification. These fluorescent proteins were further used to construct calibration standards for quantitative super-resolution microscopy. Second, the theory of qPALM was refined to accurately determine oligomeric states in proteins and an automated tool for qPALM analysis was developed. Third, qPALM was applied to investigate the oligomerization of the MET receptor tyrosine kinase. A stable cell line of MET-mEos4b was generated with the CRISPR/Cas12a technique. The receptor stoichiometry was determined in resting and ligand-treated cells. The dynamics of MET was measured in live cells, and modes of diffusion were extracted. Finally, this thesis aimed at generating an automated tool for qPALM analysis to benefit from computational advantages such as analysis speed. 


\section{Theory}

This chapter covers the theory that underlies the experiments of this thesis. First, the general principle of fluorescence is explained. Fluorescent molecules, such as fluorescent proteins, are used to label and visualize target proteins. Various labeling strategies are presented, with a particular focus on the CRISPR/Cas approach. Subsequently, the basics of fluorescence microscopy such as different illumination schemes and the diffraction limit of light are introduced. Several strategies have been developed to bypass the diffraction limit of light, which are referred to as super-resolution microscopy. One of these strategies is single-molecule localization microscopy (SMLM), which is based on the temporal separation of fluorescent emitters. Temporal separation of fluorophores can for example be achieved using photoactivatable or photoconvertible fluorescent proteins and thus enable a precise determination of fluorophore localization. Photoactivated localization microscopy (PALM) is particularly highlighted here with a focus on quantification. Finally, the theoretical background on single-particle tracking is introduced.

\subsection{Absorption and fluorescence}

In classical electrodynamics, light is described as an electromagnetic wave. However, quantum physics found that light can be described as a wave and a particle, termed the wave-particle dualism (Einstein 1905). Light is quantized and consists of small energy packages, which are described by Planck's constant $(h)$ (Planck 1901) (equation 1, where $c$ is the speed of light).

$E=h \cdot v=\frac{h \cdot c}{\lambda}$

The interaction of light with matter can be described by various phenomena such as reflection, scattering, refraction, and absorption. Absorption takes place when the energy of a photon corresponds to the energy difference between an excited and the ground state of a molecule or atom (equation 2).

$\Delta E=E_{1}-E_{0}=h \cdot v$ 
The Beer-Lambert law (equation 3) relates the concentration $(c)$, the path length $(d)$ and the molecular extinction coefficient $(\varepsilon)$ of a substance to its absorption (Beer 1852).

$A=\log \left(\frac{I_{0}}{I}\right)=\varepsilon_{\lambda} \cdot c \cdot d$

The molar extinction coefficient is specific for each substance and describes the attenuation of light at a given wavelength. The entire process of absorption and subsequent relaxation processes are described in the Jablonski diagram (Figure 2.1) (Jablonski 1933). Upon irradiation with light, a photon is absorbed, and an electron is excited from e.g. the highest occupied molecular orbital (HOMO) into the lowest unoccupied molecular orbital (LUMO). This excitation process happens without a displacement of the nuclei (Franck-Codon principle) (Franck und Dymond 1926; Condon 1928).

After excitation, internal conversion and vibrational relaxation take place, in which the molecule relaxes into the lowest vibrational state of the first excited state by non-radiative processes e.g. interaction with the environment. Subsequently, the molecule emits a photon, a process called fluorescence. This happens out of the first excited state (Kasha's rule) (Kasha 1950), and the electron returns into the electronic ground state. The energy of the emitted photon is lower than the absorbed energy, leading to a red shift of the emission spectrum compared to the absorption spectrum (Stokes shift) (Stokes 1852). Electrons in the first excited state can also undergo a spin-forbidden transition into the triplet state (intersystem crossing). From this state the electron can either relax without radiation by intersystem crossing or emit a photon, a process called phosphorescence. While excitation happens very fast $\left(10^{-15} \mathrm{~s}\right)$, internal conversion $\left(10^{-12} \mathrm{~s}\right)$ and fluorescence $\left(10^{-8} \mathrm{~s}\right)$ have longer timescales. Phosphorescence is even slower, ranging from $10^{-6} \mathrm{~s}$ to minutes (Lakowicz 2006). 


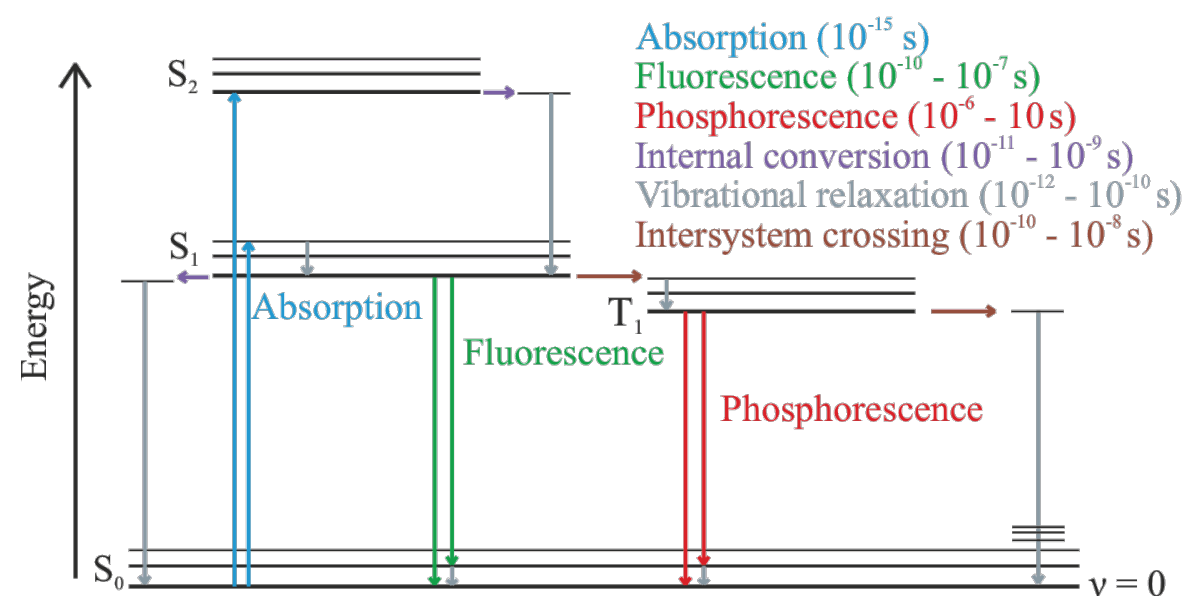

Figure 2.1: Jablonski diagram. Upon absorption of a photon, an electron can be excited from the electronic ground state $\left(S_{0}\right)$ to higher electronic singlet state (e.g. $S_{1}, S_{2}$ ) (blue). By nonradiative processes such as internal conversion (purple) and vibrational relaxation (grey), the molecules in upper electronic and vibrational states quickly reach the lowest vibrational state of the first excited state. To reach the electronic ground state, electrons can either relax nonradiatively via internal conversion, emit fluorescence (green) or transit into the triplet state $\left(\mathrm{T}_{1}\right)$ via intersystem crossing (brown) to finally reach the ground state by phosphorescence (red) or an additional intersystem crossing.

\subsection{Fluorescent probes}

Fluorescence can be used to study various characteristics of proteins by labeling them with fluorescent probes. Such fluorescent markers should have high brightness, a suitable fluorescence lifetime, high photostability, be relatively small, non-toxic to cells and easy to attach to biomolecules. The brightness of the fluorophore is a product of the extinction coefficient and the quantum yield of the fluorophore. The quantum yield of a fluorescent molecule is defined as the emitted photons divided by the absorbed photons. The fluorescence lifetime is defined by the average time the electron spends in the excited state before relaxing to the electronic ground state (Lakowicz 2006).

Almost all native proteins lack a bright chromophore that allows specific detection by fluorescence microscopy. Hence, fluorescent probes are required that are specifically attached to these proteins and enable their visualization in cells (Toseland 2013). Different classes of fluorescent probes exist including organic fluorophores and fluorescent proteins. 


\subsubsection{Organic fluorophores}

Organic fluorophores are small molecules (size of $\sim 1-2 \mathrm{~nm}$ ) that contain a conjugated $\pi$ electron system and exhibit a high brightness. Common classes of organic fluorophores include rhodamines, carbocyanines, oxazines, and carbopyronines with examples such as Alexa Fluor 647, ATTO 647N, Cy3, and Cy5. These organic fluorophores are attached to biomolecules by chemical reactions. (Heilemann et al. 2009; van de Linde et al. 2013). Organic fluorophores are conjugated to antibodies and serve to visualize proteins in cells by immunofluorescence. Furthermore, click-chemistry can be used for labeling (Horisawa 2014).

\subsubsection{Fluorescent proteins}

The discovery of fluorescent proteins enabled specific observation of proteins in cells (Shaner et al. 2005). Genetic engineering allowed target protein labeling with fluorescent proteins. Nowadays, fluorescent proteins are intensively used as fluorescent markers in in vivo studies to analyze the localization, composition, or dynamics of cellular structures (Chudakov et al. 2010).

The first discovered and most common utilized fluorescent protein was GFP, which was discovered by Osamu Shimomura in 1962 (Shimomura et al. 1962). Its complementary DNA was first isolated by Prasher in 1992 (Prasher et al. 1992) and first used as a fluorescent label in 1994 (Heim et al. 1994). GFP originates from the jellyfish Aequorea victoria, consists of 238 amino acids and has a molecular weight of about $27 \mathrm{kDa}$ (Slade et al. 2009). It has a self-organized chromophore consisting of the three amino acids Ser65, Tyr66, and Gly67, which is protected within a $\beta$-barrel structure (Yang et al. 1996) (Figure 2.2). Fused to a proteins of interest, GFP enabled the localization of many proteins in cells (Ellenberg et al. 1998; Ghaemmaghami et al. 2003; Kalir und Alon 2004; Xie et al. 2008). Besides GFP, many other fluorescent proteins were discovered and developed such as YFP (Ormö et al. 1996) and mCherry (Shaner et al. 2004) among others. 


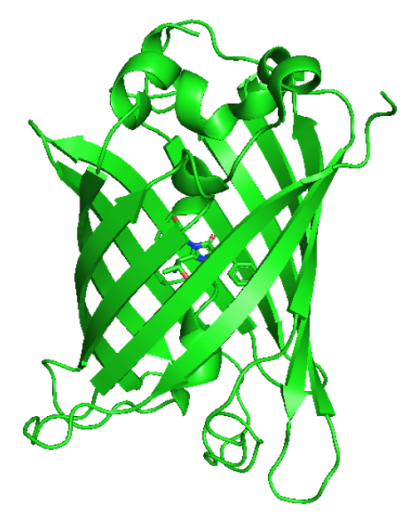

Figure 2.2: Crystal structure of GFP. The structure was taken from the protein data bank (PDB: 4KW4) (Barnard et al. 2014).

Single-molecule localization microscopy requires photoactivatable (Lukyanov et al. 2005), photoswitchable (Zhou und Lin 2013), or photoconvertible (Baker et al. 2010) fluorescent proteins (Figure 2.3) (Fürstenberg und Heilemann 2013). Photoactivatable fluorescent proteins are initially in a non-fluorescent state. When irradiated with violet light, decarboxylation of e.g. Glu222 leads to the photoactivated species by forming the conjugated $\pi$-electron system (Shcherbakova und Verkhusha 2014) (Figure 2.3 A). Examples for photoactivatable fluorescent proteins are PAGFP (Patterson und Lippincott-Schwartz 2002) and PAmCherry (Subach et al. 2009). Dronpa is one of the commonly used photoswitchable fluorescent proteins (Ando et al. 2004). Here, a reversible switch between a dark and a bright state is provoked by cis-trans isomerization and/or protonation by exposure to $405 \mathrm{~nm}$ light (Zhou und Lin 2013) (Figure $2.3 \mathrm{~B}$ ). Photoconvertible fluorescent proteins possess two (or more) fluorescent states (Figure 2.3 C). Irradiation with violet light induces an irreversible cleavage of the protein backbone, which leads to a prolonged $\pi$-electron system (Wachter 2017). As a result, the fluorescent protein changes its spectral characteristics into red-shifted absorption and emission spectra. Members of the Eos family are frequently used photoconvertible fluorescent proteins in PALM (Wiedenmann et al. 2004; McKinney et al. 2009; Zhang et al. 2012; Paez-Segala et al. 2015). 
A

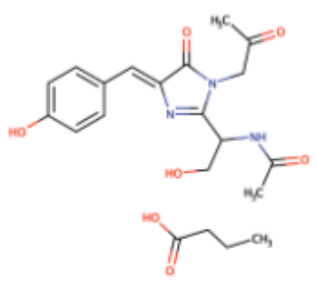

(dark)

Photoactivation

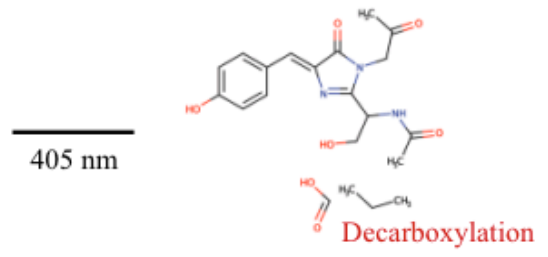

(fluorescent)

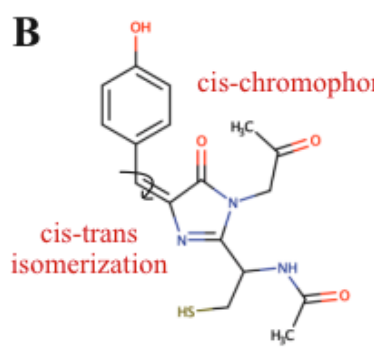

(dark)

C

Photoswitching

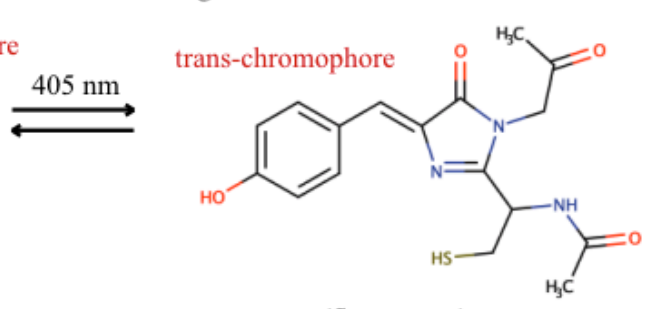

(fluorescent)

Photoconversion

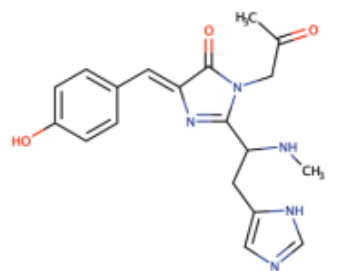

(fluorescent 1)

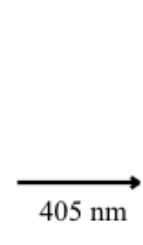

$405 \mathrm{~nm}$

(fluorescent 2)

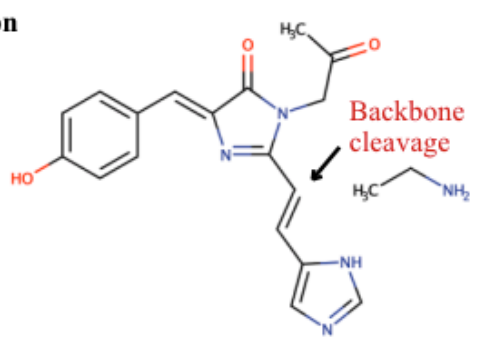

Figure 2.3 Mechanism of photoactivation, -switching and -conversion of fluorescent proteins. (A) Photoactivation of fluorescent proteins involves decarboxylation induced by irradiation with violet light. (B) Violet light triggers photoswitching of fluorescent proteins by cis-trans isomerization. (C) Irreversible photoconversion occurs by backbone cleavage induced by violet irradiation.

The Eos family evolved from the tetrameric EosFP from the stony coral Lobophyllia hemprichii (Wiedenmann et al. 2004). Mutations of the amino acids V123T and T158H led to the discovery of mEos, a mostly monomeric form of EosFP (Wiedenmann et al. 2004). The brightness was improved by further mutations (N11K; E70K; H74N; H121Y) and led to the development of mEos2 (McKinney et al. 2009), which still showed a slight tendency to form dimers and tetramers in dense samples (Hoi et al. 2010) (Figure 2.4). The brightness was increased even further in mEos3.2, where further mutations also led to increased monomeric performance (Zhang et al. 2012). The currently newest and most optimized version is mEos4b (M1V; K9R; F34Y; S39T; A69V; C195A), which was demonstrated to be suitable for correlative fluorescence and electron microscopy (PaezSegala et al. 2015). 


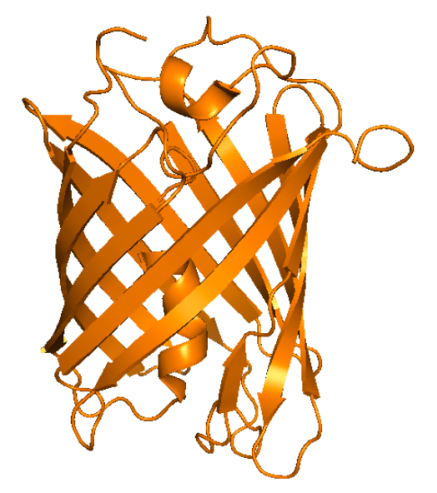

Figure 2.4: Crystal structure of mEos2. The structure was taken from the protein data bank (PDB: 3S05) (Zhang et al. 2011).

\subsection{Fluorophore labeling strategies}

Fluorescence microscopy requires the attachment of fluorescent markers to target biomolecules. The labeling method for attaching a fluorescent probe to a target protein must be carefully considered with respect to important parameters such as specificity, degree of labeling, efficiency and background signal (off-target effects). However, the choice of the labeling technique mostly depends on the respective application. The following methods will be explained in the following sections: (i) immunofluorescence, (ii) labeling strategies with reactive groups, (iii) transfection of plasmids, and (iv) CRISPR/Cas.

\section{Immunofluorescence}

One strategy to label target proteins with organic fluorophores is immunofluorescence. In immunofluorescence, organic fluorophores are covalently bound to antibodies, which in turn bind specifically to their target epitopes (Figure 2.5 A) (Suzuki et al. 2007). A distinction between direct and indirect immunofluorescence is made (Aoki et al. 2010). Direct immunofluorescence uses primary antibodies that carry the organic fluorophore and target a protein of interest (Coons et al. 1941; Coons und KAPLAN 1950). In indirect immunofluorescence, fluorophore-labeled secondary antibodies bind to unlabeled primary antibodies (Lewis Carl et al. 1993). A major advantage of immunofluorescence is the high specificity of antibody-antigen interaction. Indirect immunofluorescence profits from additional signal strength, as multiple antibodies can bind to the primary antibody. In addition, indirect immunofluorescence allows a higher flexibility in experimental design and thus is cheaper. However, indirect immunofluorescence is 
limited by the number of available species restricting multiplexing experiments to a minimum of $\sim 4$ different targets. In addition, cross-activities between different antibody species complicate the analysis of multiple proteins. A disadvantage of immunofluorescence in general is the large size of the antibody (10-15 nm), which introduces a spatial displacement of the fluorophore from the target (Huang et al. 2010).

\section{Labeling strategies with reactive groups}

A direct labeling of the target protein with organic fluorophores is achieved by chemical modification of lysines or N-terminal amino groups with an organic fluorophore coupled to an N-hydroxysuccinimide (NHS) ester (Figure 2.5 B). The ester modification is highly reactive and forms a peptide bond to the respective amino group (Koniev und Wagner 2015). A major advantage is the direct coupling to the target protein, which creates almost no label displacement and thus is suited for very precise spatial determinations. In addition, the target protein is often labeled with multiple fluorophores, since lysines are abundant amino acids, creating a strong signal. However, this is a major disadvantage in quantification as no defined labeling stoichiometry is obtained. Maleimide-coupled fluorophores bind to cysteines and represent an alternative, with cysteines occurring less frequently compared to lysines (Toseland 2013). This labeling strategy is favored for proteins possessing cysteines, but cysteines can also be inserted by e.g. amber mutations (Heil et al. 2018). Amber mutations are point mutations that lead to a stop codon. By feeding cells with special tRNAs that code for the stop codon, synthetically modified amino acids can be incorporated into the protein sequence and enable specific labeling.

A

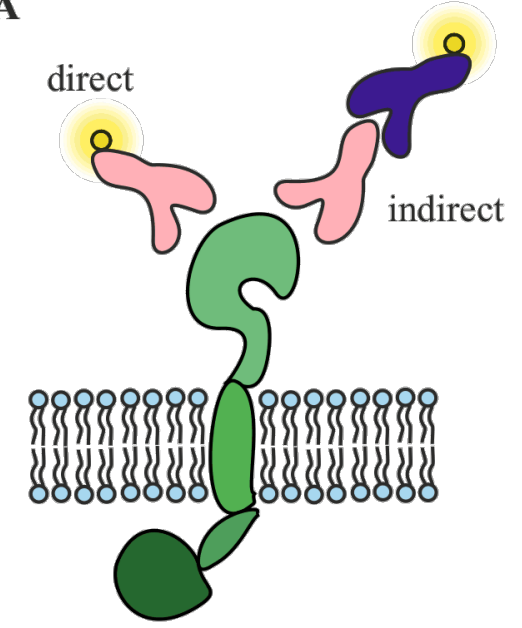

B

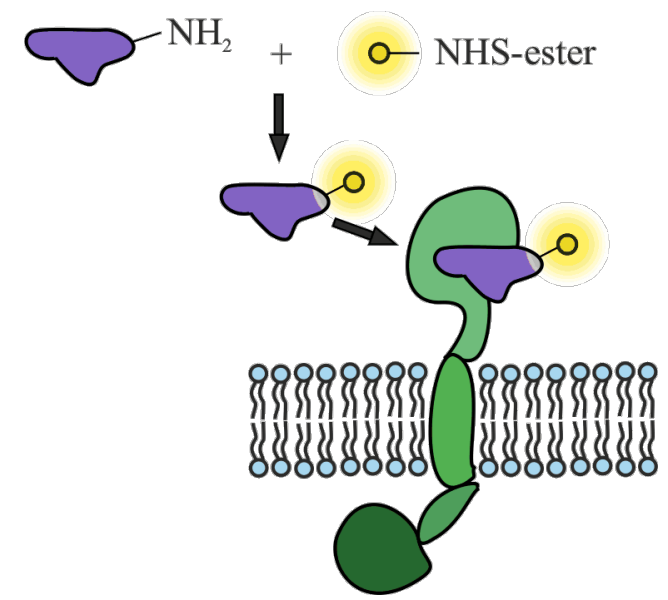

Figure 2.5: Labeling schemes of target proteins with organic fluorophores. (A) Direct or indirect immunofluorescence stains the target protein with fluorophore-labeled antibodies. (B) 
The target protein or binding partner (purple) contains an amino group $\left(\mathrm{NH}_{2}\right)$ with which it can be coupled to fluorophores (yellow) containing an NHS-ester modification.

\section{Genetic engineering}

Fluorescent proteins are attached directly to a target protein, usually in the N- or Cterminal region. The proteins are genetically encoded as fusion proteins, whereby the protein of interest is co-expressed with the desired fluorescent protein. This facilitates a one-to-one stoichiometry of the label and the target molecule, which is a major advantage over other labeling techniques, especially for quantitative microscopy.

One way to insert the DNA sequence of the fusion protein into mammalian cells is by transfection of plasmids containing the engineered DNA sequence (Figure $2.6 \mathrm{~A}$ ). Numerous cloning strategies have been developed to produce artificial plasmids, such as cloning with standard restriction enzymes or cloning using Gibson assembly (Gibson et al. 2009; Bertero et al. 2017; Ortega et al. 2019). However, transfection efficiency can vary greatly between cells, resulting in different expression levels of the target protein. This is a major disadvantage, since overexpression of proteins has been shown to influence protein organization and function in a cell (Lisenbee et al. 2003; Doyon et al. 2011; Gibson et al. 2013).

In contrast, genomic engineering techniques such as CRISPR/Cas (Figure 2.6 B) ensure an endogenous expression level of the target protein (Ran et al. 2013; Fueller et al. 2020). The DNA sequence of the fluorescent protein can be inserted directly in the genomic DNA at the N- or C-terminus. Protein expression is regulated by the native promotor. Each of the cells contain the same genomic information, which leads to a homogenous and endogenous protein density.

A

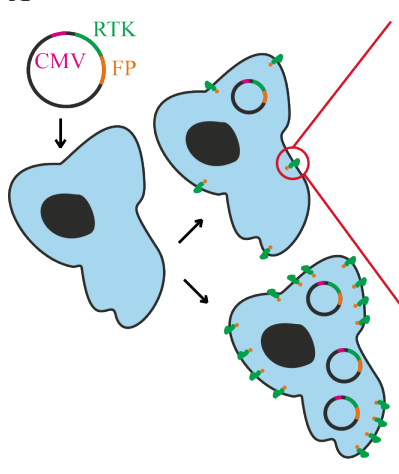

B

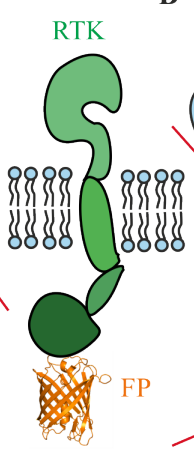

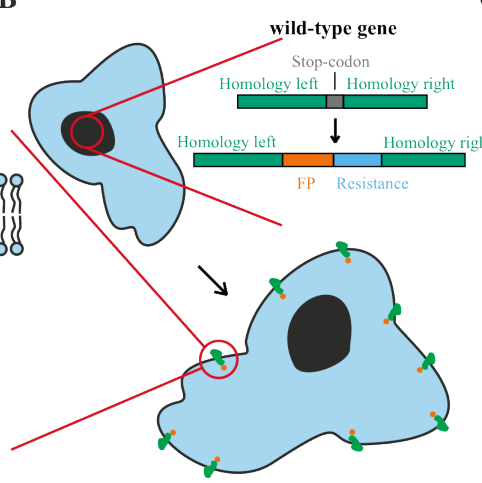

C

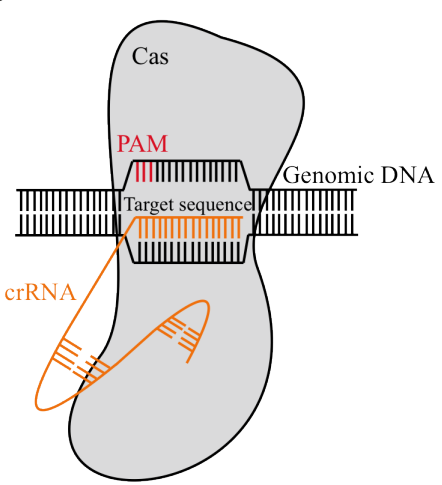

Figure 2.6: Labeling schemes of target proteins with fluorescent proteins. (A) Fusion proteins consisting of the target protein (e.g. RTK, green) and a fluorescent protein (FP, orange, PDB: 
3S05) can be introduced into cells by transfection of plasmids to study the target protein. (B) CRISPR/Cas enables the genomic labeling of target proteins with a FP (orange) and introduction of a resistance marker for selection (blue). In C-terminal tagging, the DNA of the tag is typically inserted directly before the stop-codon (grey). (C) The Cas enzyme forms a complex with the crRNA and anneals to the corresponding target sequence on the genomic DNA. The PAM sequence consists of about 3-4 base pairs and serves as recognition sequence for the Cas enzyme to cut the genomic DNA.

\subsubsection{CRISPR/Cas system}

CRISPR/Cas is a molecular biology tool which enables the knockdown of certain proteins as well as their specific labeling with fluorescent proteins (Pickar-Oliver und Gersbach 2019; Banan 2020). The method originated from the discovery of clustered regularly interspaced short palindromic repeat (CRISPR) sequences that are part of the adaptive immune system in bacteria. CRISPR/Cas was established between 1987 and 2012 due to contributions of many scientists (Ishino et al. 1987; Mojica et al. 2000; Mojica et al. 2005; Pourcel et al. 2005; Bolotin et al. 2005; Makarova et al. 2006; Barrangou et al. 2007; Brouns et al. 2008; Marraffini und Sontheimer 2008; Hale et al. 2009; Garneau et al. 2010; Deltcheva et al. 2011; Sapranauskas et al. 2011; Gasiunas et al. 2012; Jinek et al. 2012). Jennifer Doudna and Emmanuelle Charpentier were awarded the Nobel Prize in Chemistry 2020 for their discovery that CRISPR/Cas can be programmed to manipulate genomic DNA.

For genome editing via CRISPR/Cas, the Cas enzyme and a targeting sequence, the CRISPR RNA (crRNA) are needed (Figure 2.6 C). The Cas enzyme is a nuclease that works like a genetic scissor. In order to enable a specific cut of the DNA, the Cas enzyme binds to a crRNA, which offers a specific sequence complementary to the site at which the double-strand break is to be produced. Additionally, a protospacer adjacent motif (PAM) sequence is required on the target DNA, which acts as a recognition sequence for the Cas enzyme. The PAM sequence is a DNA sequence consisting of 2-6 base pairs, which has to be located directly next to the homologous crRNA for the Cas enzyme to specifically cut the genomic DNA. After the double-strand break is introduced, the DNA is repaired by non-homologous end-joining (NHEJ) or homology-directed repair (HDR). NHEJ can lead to a knockdown of the protein, for example due to raster shifts or earlier stop codons. In order to insert a gene sequence through HDR, a homologous template DNA is additionally required. The homologous template DNA has a complementary 
DNA sequence to the genetic sequence of interest and can therefore be incorporated into the genome after the double-strand break occurred (Pickar-Oliver und Gersbach 2019). In summary, CRISPR/Cas requires only a few steps to obtain genetically modified cells. The most widespread and frequently used Cas enzyme is Cas9. Besides Cas9, there are several other Cas enzymes (Pickar-Oliver und Gersbach 2019), such as the Cas 12 enzyme (Zetsche et al. 2015). Cas12, also known as Cpf1, is a single RNA-guided endonuclease that lacks the trans-activating crRNA and is part of the class 2 CRISPR/Cas system (Zetsche et al. 2015). In contrast to Cas9, the application of the Cas 12 enzyme leads to single strand breaks that results in sticky ends instead of blunt ends. Advantages of Cas 12 are a higher specificity in vivo (Kleinstiver et al. 2016; Kim et al. 2017) and cutting the genomic DNA at a distinct distance from the recognition sequence (Zetsche et al. 2015; Moreno-Mateos et al. 2017), thus yielding higher tagging efficiencies.

\subsubsection{CRISPR/Cas12a-assisted genomic labeling}

A drawback of the CRISPR/Cas genomic engineering described above is the timeconsuming synthesis of a homologous template. A time efficient approach of the CRISPR technique, namely polymerase chain reaction (PCR) tagging, uses engineered plasmids to generate the homologous templates (PCR cassettes) (Fueller et al. 2020) (Figure 2.7). PCR tagging uses Cas12a to label the C-terminus of proteins through e.g. fluorescent proteins. Specially designed primers anneal on engineered plasmids to generate the homologous template DNA in a single PCR reaction. After the PCR reaction, the PCR product is purified and transfected into mammalian cells together with a plasmid containing the Cas12a enzyme. Antibiotic resistances on the PCR cassette specifically select those cells which were successfully transfected with the PCR cassette. Thus, in only a few steps, cell lines can be generated that carry the desired tag fused to the target protein. For example, fluorescent proteins can be attached to target proteins to investigate their specific localization in the cell by fluorescence microscopy. 


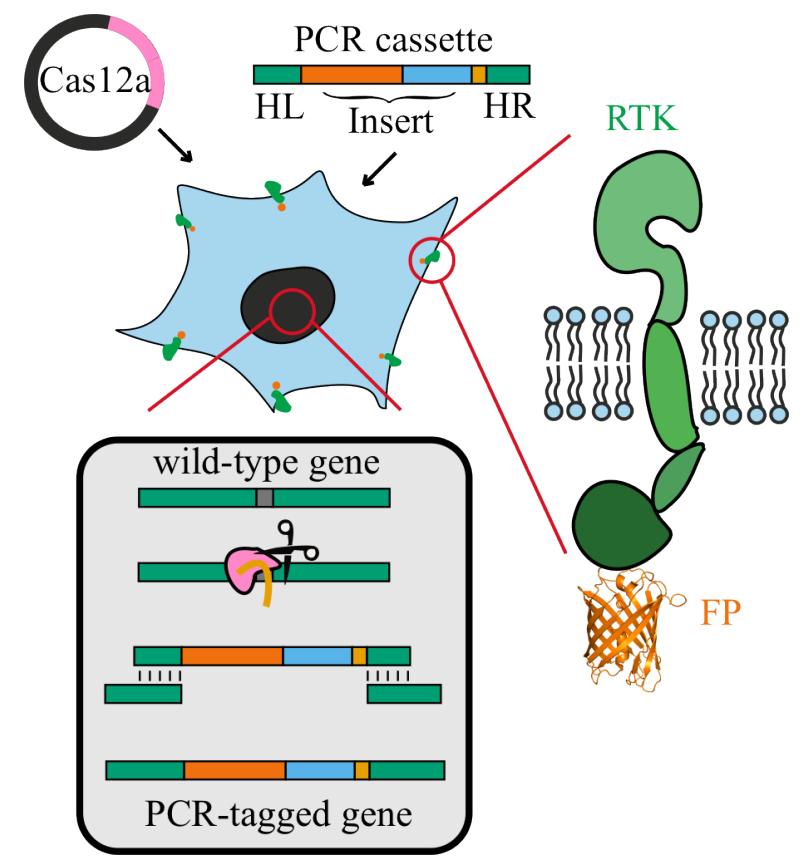

Figure 2.7: CRIPSR/Cas12a-assisted PCR tagging for genome labeling. CRISPR/Cas12aassisted PCR tagging requires the Cas12a expression vector and a PCR cassette that are transfected into the cells of interest. The wild-type gene is cut by Cas12a and the PCR cassette is inserted into the genome resulting in the expression of a fusion protein consisting of the target protein (green) and e.g. a fluorescent protein (orange, PDB: 3S05).

\subsection{Fluorescence microscopy}

Fluorescence microscopy has revolutionized cell biology by providing insights into the function of biomolecules in cells at sub-cellular levels (Combs 2010). The method offers a high sensitivity by detecting specific biomolecules as single particles in exceptionally small quantities. To enable specific detection of biomolecules, fluorescent probes (chapter 2.2) are attached to the biomolecule of interest. By specific excitation and emission of the fluorescent probe, diverse processes including location, association, and dynamics of proteins in living cells can be analyzed. In addition, several structures and proteins can be analyzed simultaneously by using fluorophores that differ in their emission spectrum.

Fluorescence microscopes are equipped with a light source to excite the fluorophores. This light source can be, for example, excitation lasers for fluorophores. The laser beam enables the illumination of the sample and is guided into the objective, which additionally collects the emission light. Different illumination schemes are used to excite and detect fluorescent molecules in biological samples. 


\subsubsection{Illumination schemes in fluorescence microscopy}

Fluorescence microscopy can be realized in widefield or confocal illumination. In widefield microscopy, the whole sample is illuminated, while in confocal microscopy, a small area is illuminated and the sample is scanned (St Croix et al. 2005). In the following, only widefield microscopy is considered, since only this technique was used in this work. In widefield microscopy, the laser beam is focused on the back focal plane of the objective (Figure $2.8 \mathrm{~A}$ ), leading to a homogeneous illumination of the sample (Lakowicz 2006). In thick samples, this leads to background fluorescence from out-of-focus image planes. To minimize background signals from areas outside the focal plane, highly inclined and laminated optical sheet microscopy (HILO) illumination can be used (Figure 2.8 B) (Tokunaga et al. 2008). In this case, the light beam is focused aside the center of the back focal plane. As a result, the sample is excited with a slightly tilted light sheet of $\mu \mathrm{m}$ thickness. This illumination scheme is especially useful to observe e.g. processes inside the cytoplasm or the nucleus, since a higher contrast than in widefield microscopy is achieved.

When monitoring biological structures near the glass surface, total internal reflection fluorescence (TIRF) illumination can be beneficial (Ambrose 1956; Axelrod et al. 1984; Axelrod 2001) (Figure $2.8 \mathrm{C}$ ). This is achieved by a shift of the excitation light on the back focal plane to one side, which leads to inclination. The shift is increased until the critical angle $\left(\theta_{\text {crit }}\right)$ is exceeded (Figure $2.8 \mathrm{C}$ ). The critical angle $\theta_{\text {crit }}$ can be determined from Snell's law by including the refractive indices $(n)$ of both media (e.g. glass, $n=$ 1.51 and water, $n=1.33$ ) and in biological samples typically is about $62^{\circ}$ (equations 4 and 5).

$\frac{\sin \left(\theta_{2}\right)}{\sin \left(\theta_{1}\right)}=\frac{n_{1}}{n_{2}}$

$\theta_{\text {crit }}=\sin ^{-1}\left(\frac{n_{2}}{n_{1}}\right)$

Total internal reflection generates an evanescent light field close to the surface that decays exponentially and has a penetration depth of about $200 \mathrm{~nm}$ (Martin-Fernandez et al. 2013). This leads to a better signal-to-background ratio because of reduced background fluorescence but limits observations to regions close to the glass surface. 
A

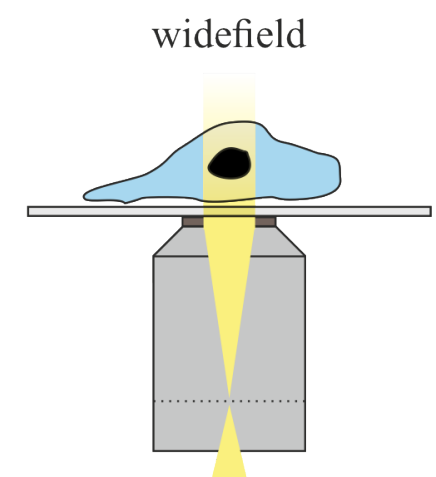

B

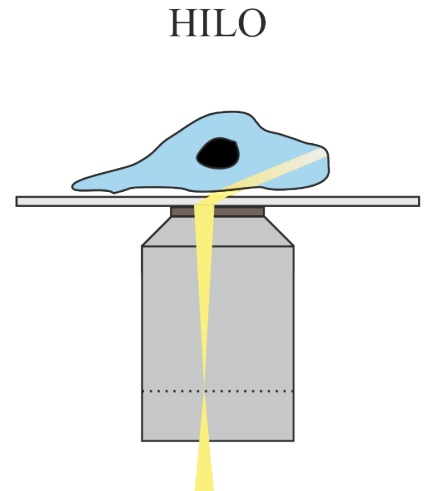

C

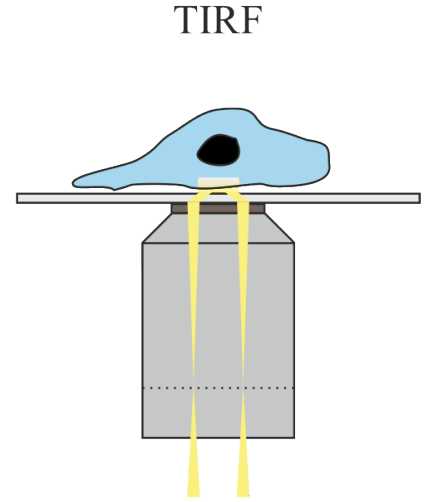

Figure 2.8: Illumination schemes of widefield, HILO, and TIRF. (A) Widefield illumination: The excitation beam is focused on the back focal plane (dotted line) and leads to a wide and homogenous illumination of the sample. (B) HILO illumination: The excitation beam is slightly shifted to one side of the back focal plane of the objective generating a light sheet that passes through the sample and thus reduces background fluorescence. (C) TIRF illumination: The excitation light hits the glass surface at an angle larger than the critical angle $\left(\theta_{\text {crit }}\right)$ leading to total internal reflection and generating an evanescent field at the surface. This leads to an excitation of molecules that are located near the glass surface.

\subsubsection{Diffraction limit}

The highest spatial resolution that can be achieved is an important experimental parameter in light microscopy. In light microscopy, spatial resolution is defined by the minimum distance that two objects must have in order to be perceived as separate structures. According to the physics of diffraction, the spatial resolution is limited due to diffraction. By observing optical gratings, Ernst Abbe was the first to describe the underlying theory (Abbe 1873). The theory demonstrates that the resolution $\left(d_{\min }\right)$ depends on the wavelength $(\lambda)$ and the numerical aperture $(N A=n \cdot \sin (\alpha), n$ : the refractive index of the medium) of the objective (equation 6).

$$
d_{\min }=\frac{\lambda}{2 \cdot N A}
$$

The fluorescence emission of a single fluorophore appears as an intensity distribution which is termed point spread function (PSF) and is mathematically described by the Bessel $(1,1)$ function (Airy 1835). In 1896, Rayleigh postulated another definition of the 
resolution limit building on the discrimination of the point spread function (PSF) of two neighboring objects (Rayleigh 1896). The Rayleigh criterion states that if the maximum of one PSF coincides with the first minimum of another PSF, the two underlying objects can just about be distinguished. The minimal lateral distance $\left(d_{\min }\right)$ in Rayleigh criterion is described by the wavelength of the light $(\lambda)$ divided by the numerical aperture (equation 7).

$d_{\min }=0.61 \frac{\lambda}{N A}$

Since fluorophores typically emit light at a wavelength between $400-600 \mathrm{~nm}$, the spatial resolution of fluorescence microscopy is limited to $\sim 200-300 \mathrm{~nm}$ (Sauer und Heilemann 2017). The size of biomolecules, such as proteins is typically around a few nanometers. Therefore, the discovery of super-resolution techniques was a breakthrough in fluorescence microscopy enabling the localization of molecules with near-molecular resolution (Huang et al. 2010; Sauer und Heilemann 2017).

\subsection{Super-resolution microscopy}

Super-resolution microscopy bypasses the diffraction limit of light microscopy and different strategies were developed in the end of the $20^{\text {th }}$ century / early $21^{\text {th }}$ century amongst others by Eric Betzig, Stefan W. Hell and William E. Moerner (Möckl et al. 2014). Two general concepts, a deterministic and a stochastic approach, are distinguished. The deterministic approach includes stimulated-emission depletion (STED), which uses a donut-shaped laser beam deactivating fluorophores overlaid with a Gaussian beam for excitation in a confocal geometry (Blom und Widengren 2017). Based on STED and stochastic emission, maximally informative luminescence excitation (MINFLUX) was invented which improves the resolution to $\sim 1-2 \mathrm{~nm}$ (Balzarotti et al. 2017). Singlemolecule localization microscopy (SMLM) is a stochastic approach that is based on the detection of a subset of single emitters (Huang et al. 2010; Sauer und Heilemann 2017).

\subsubsection{Single-molecule localization microscopy}

SMLM is an approach in which PSFs of spatially nearby fluorophores are separated in time by stochastic activation, switching or transient binding. The localization of single molecules by approximating their center of their PSFs achieves nanometer precision. 
Determining the center of the PSF for all fluorescent signals of an imaging experiment allows the reconstruction of a super-resolved image (Figure 2.9) (Sauer und Heilemann 2017).

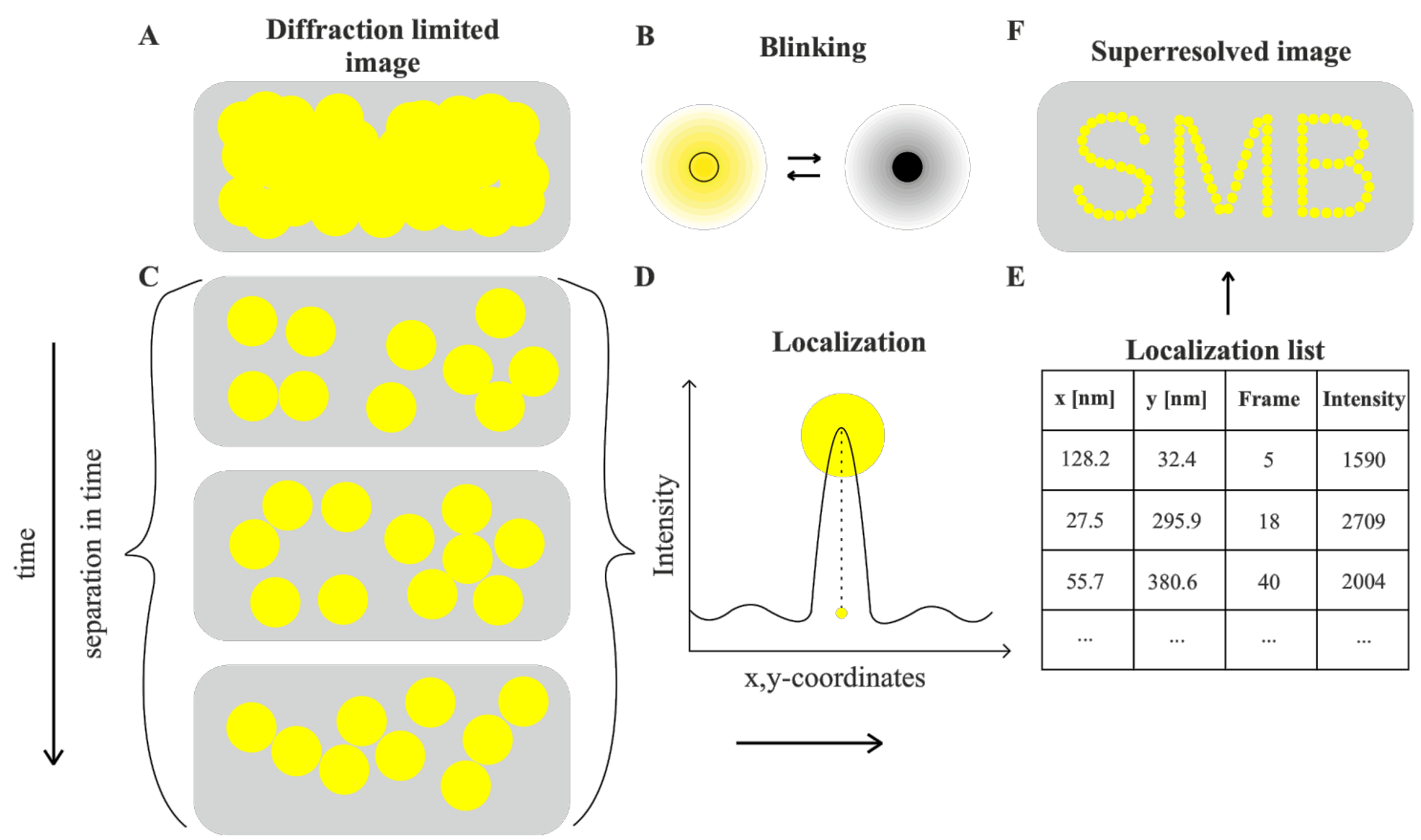

Figure 2.9: Principle of single-molecule localization microscopy. (A) Schematic illustration of a diffraction-limited image. (B) SMLM is achieved by e.g. photoswitching the fluorophores. (C) The detection of only a subset of fluorescent emitters in each frame allows the elimination of overlapping PSFs. (D) The position of each emitter is determined by approximating the intensity profile with a Gaussian function. (E) The localization list contains information of the fluorescence signals such as the position, the time and intensity. (F) Reconstruction of a super-resolved image from all localizations.

The precision with which the position of a molecule can be determined depends on the number of detected photons ( $\left.\mathrm{N}_{\text {photons }}\right)$, the standard deviation of the widefield PSF $\left(\sigma_{P S F}\right)$, the background noise $(b)$ and the pixel size $(a)$ according to equation 8 (Mortensen et al. 2010):

$\sigma_{\text {loc }}=\sqrt{\frac{\sigma_{P S F}^{2}}{N_{\text {Photons }}} \cdot\left(\frac{16}{9}+\frac{8 \pi \sigma_{P S F}^{2} b^{2}}{N_{\text {Photons }} a^{2}}\right)}$

The localization precision can also be determined experimentally with the help of a nearest neighbor based analysis (NeNA) (Endesfelder et al. 2014). This strategy 
calculates the distance between fluorescence signals of the same emitter in consecutive frames. The localization precision is obtained by approximating the distance distribution $(p(d))$ with the respective amplitude $\left(A_{n}\right)$, the standard deviation $(\omega)$, and a maximum distance $\left(d_{c}\right)$ of the Gaussian distribution (equation 9$)$ :

$p(d)=A_{1} \cdot\left(\frac{d}{2 \sigma^{2}} \cdot e^{\frac{-d^{2}}{4 \sigma^{2}}}\right)+A_{2} \cdot\left(\frac{1}{\sqrt{2 \pi \omega^{2}}} \cdot e^{\frac{-\left(d-d_{c}\right)^{2}}{2 \omega^{2}}}\right)+A_{3} \cdot d$

With SMLM, localization precisions in the range of 5-20 $\mathrm{nm}$ are achieved (Sauer und Heilemann 2017; Schnitzbauer et al. 2017).

SMLM includes microscopy techniques such as stochastic optical reconstruction microscopy (STORM) (Rust et al. 2006), direct STORM (dSTORM) (Heilemann et al. 2008), photoactivated localization microscopy (PALM) (Betzig et al. 2006), points accumulation for imaging in nanoscale topography (PAINT) (Sharonov und Hochstrasser 2006), and DNA-PAINT (Jungmann et al. 2010) (Figure 2.10).

The temporal separation of fluorescence signals in $d$ STORM is based on reversible photoswitching of organic fluorophores (Figure 2.10 A). The photoswitching is enabled by buffers containing reducing thiols, such as $\beta$-mercaptoethylamine (MEA), generating long-lived dark states in fluorohpores. Only a small subset of molecules fluoresces at each time point. Since organic fluorophores are bright and many photons per fluorophore are obtained, a high resolution of $\sim 10 \mathrm{~nm}$ can be achieved (Sauer und Heilemann 2017).

DNA-PAINT uses reversible binding of fluorophore-labeled oligonucleotides (imager strands) to complementary target strands (docking strands), which are coupled to the structure of interest (Figure 2.10 B). Since only a subset of the imager strands is bound to the docking strands and thus excited and detected, the individual localizations are temporally separated. Due to the large pool of imager strands, the individual binding sites can be localized multiple times, resulting in a high spatial resolution $(\sim 5 \mathrm{~nm})$ (Schnitzbauer et al. 2017).

The labeling of target proteins for $d$ STORM and DNA-PAINT experiments typically involves antibodies. This complicates the observation of proteins in live cell experiments, since antibodies are not able to cross the plasma membrane. Live cell SMLM is facilitated with PALM, which uses photoactivated, photoswitchable or photoconvertible fluorescent proteins that are genetically fused to the target protein. These proteins are stochastically 
photoactivated by violet light to enable small emitter densities (Figure 2.10 C) (Betzig et al. 2006).

A

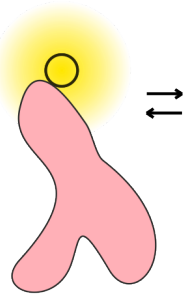

ON-state

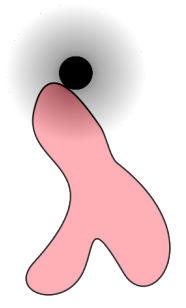

OFF-state
B

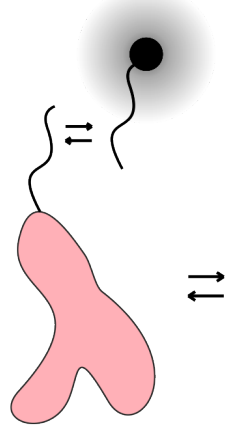

OFF-state

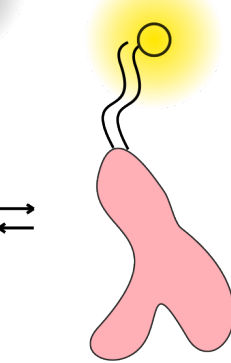

ON-state
C

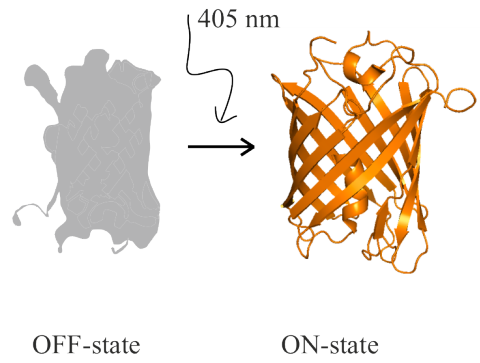

PALM

dSTORM

DNA-PAINT

Figure 2.10: Overview of common SMLM techniques. (A) In $d$ STORM experiments, fluorophore-labeled antibodies that switch between a fluorescent ("ON") and a dark ("OFF") state are used. (B) Illustration of DNA-labeled antibodies for DNA-PAINT experiments. Fluorescencelabeled DNA strands are solely detected when bound to the complementary, stationary DNA strands attached to the antibodies. (C) In PALM, a photoactivated fluorescent protein is photoactivated by violet light from an inactive ("OFF") state into a fluorescent (“ON") state.

\subsubsection{Photoactivated localization microscopy}

Photoactivated localization microscopy is a SMLM technique that takes advantage of the photoactivation or photoconversion process of fluorophores (Betzig et al. 2006). At the beginning of a PALM experiment, all fluorophores are either non-fluorescent or emit at a different wavelength. Irradiation with violet or ultraviolet light $(\sim 380-405 \mathrm{~nm})$ stochastically converts the fluorescent protein into an active, fluorescent state. A low emitter density is ensured by using low illumination intensity (405 nm) activating only a small subset of the fluorophores and continuous photobleaching. A localization precision of $\sim 10 \mathrm{~nm}$ can be achieved with PALM microscopy (Shroff et al. 2013).

Fluorescent proteins are genetically fused with the protein of interest by e.g. genomic editing, which allows stoichiometric labeling of intracellular proteins without the need for cell membrane permeabilization. Therefore, cellular structures remain mostly unaffected, so that a native environment is guaranteed. Additionally, stoichiometric labeling facilitates quantitative SMLM and reports on the nano-organization of proteins and complexes in cells, which is one of the most challenging tasks in super-resolution microscopy (Dietz und Heilemann 2019). 


\subsection{Quantitative SMLM}

The quantitative analysis of the organization of proteins into functional units is a key point in understanding the underlying principles and processes of cell biology. SMLM can report on protein numbers in complex oligomers by resolving nano-scale protein clusters in cells. However, quantitative approaches pose a major challenge, since protein numbers are overestimated by repeated periods of fluorescence emission and underestimated by a fraction of inactive fluorophores (Lee et al. 2012). Recently, SMLM methods were extended to quantification of target structures by analyzing kinetic and statistical processes (Dietz und Heilemann 2019).

One way to achieve molecular quantification is to analyze the average number of fluorescence emission events per single emitter, which can serve as calibration for quantification of protein assemblies (Puchner et al. 2013; Nan et al. 2013; Finan et al. 2015) (Figure 1.2 A,B). An alternative strategy is pursued in pair-correlation analysis, which in addition to protein numbers reports on cluster size and cluster densities (Sengupta et al. 2011) (Figure 1.2 C). The method explores the probability of finding a second fluorescent signal in a defined distance from an initial fluorescence event. Paircorrelation functions describe the correlation between the distances of these emission events. This method was applied to different proteins such as VSVG, Lyn, and LAT (Sengupta et al. 2011; Sherman et al. 2011).

An approach based on the intrinsic kinetics of repetitive binding events of DNA strands is quantitative DNA-PAINT (qPAINT) (Figure 1.2 A,E). The binding frequency of DNA strands is proportional to the number of binding sites and hence may report on target stoichiometry. A constant imager strand concentration allows predictions on different $\mathrm{k}_{\text {off }}$ rates of reference and target structures for quantification. For example, qPAINT was applied to quantify Nup98 protein clusters in the nuclear pore complex (Jungmann et al. 2016), ryanodine receptors in calcium release sites of cardiomyocytes (Jayasinghe et al. 2018), and the number of AMPA receptors in synapses of dendrites (Böger et al. 2019). A correction for undetected molecules or rather docking sites is missing in both, paircorrelation and qPAINT analysis, leading to imprecise protein numbers, which most likely are underestimated.

A quantitative analysis based on PALM (qPALM) investigates the distribution of number of blinking (recurrence of fluorescence) events and includes a term for inactive molecules (Figure 1.2 A,D). This facilitated the reliable determination of protein numbers in 
reference proteins (Fricke et al. 2015; Hummer et al. 2016). qPALM is based on a 4-state stochastic model that describes the photophysics of photoactivatable and photoconvertible fluorescent proteins (Figure 2.11 A) (Annibale et al. 2010). After activation, the fluorescent protein switches into a reversible dark state or photobleaches irreversibly. The probability of the fluorescent protein being photobleached is defined as the bleaching probability $p$. The number of transitions into the dark state and back to its fluorescent state is referred to as the number of blinking events $n$ (Figure $2.11 \mathrm{~B}$ ).

The knowledge of the photophysical model led to the discovery that the number of blinking events follows a geometric distribution (Lee et al. 2012). In addition, a further study implemented a $q$-value into the stochastic model, which corrects for inactive fluorophores (Hummer et al. 2016). This allowed the description of the distribution of the number of blinking events for oligomers of different orders $(m)$ (Figure $2.11 \mathrm{C}$ ). The blinking properties of monomers $(m=0)$ and dimers $(m=1)$ can be thus described by the following geometric distributions (equations 10-12):

$$
\begin{aligned}
& p_{m}(n)=\sum_{k=0}^{\min (m, n)} p^{k+1}(1-p)^{n-k} q^{m-k}(1-q)^{k}\left(\begin{array}{l}
m \\
k
\end{array}\right)\left(\begin{array}{l}
n \\
k
\end{array}\right) \\
& p_{0}(n)=p \cdot(1-p)^{n} \\
& p_{1}(n)=p(1-p)^{n-1}[n p(1-q)+q(1-p)]
\end{aligned}
$$

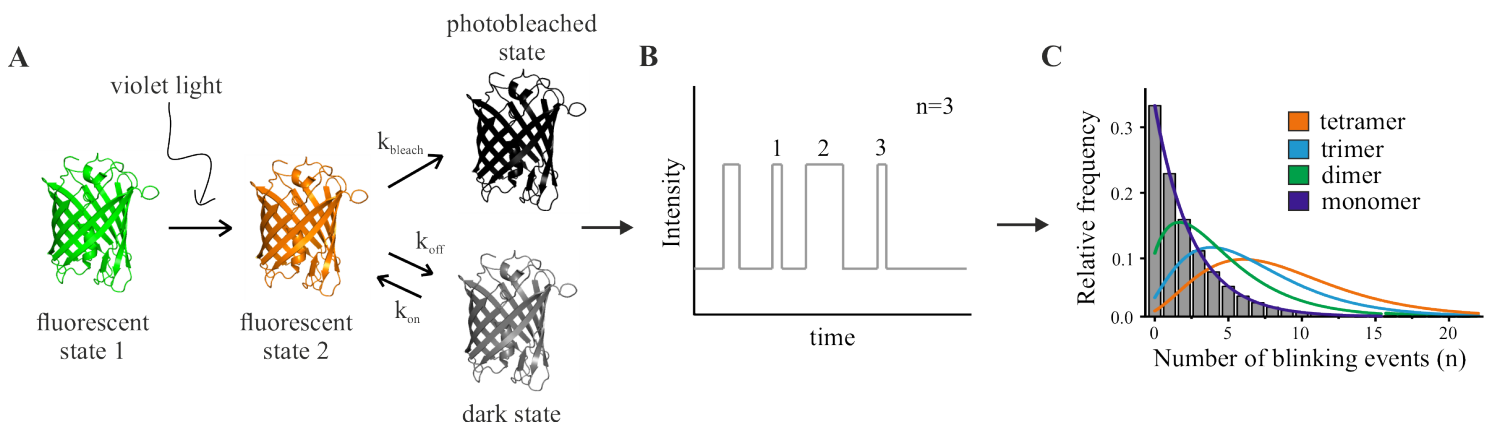

Figure 2.11: Counting of photoconvertible fluorescent proteins with qPALM. (A) 4-state stochastic model of photoconvertible fluorescent proteins, which describes the kinetics of photoconvertible fluorescent proteins (PDB: 3S05). The fluorescent protein is switched by violet light into a different fluorescent state from which it can reversibly switch into a dark state or photobleach irreversibly. (B) Intensity-time trace of a fluorescent protein showing three blinking events. (C) Histogram of the number of blinking events for a monomeric fluorescent protein. Monomer (dark blue), dimer (green), trimer (light blue) and tetramer (orange) functions are shown. 


\subsection{Single-particle tracking}

Understanding the dynamics of biomolecules in cellular processes is of fundamental interest to investigate and uncover functional mechanisms in cell biology. Single-particle tracking reports on such dynamics of biomolecules by following the movement of single, fluorescently labeled molecules. The dynamics of individual particles are described by the diffusion coefficient $D$. The diffusion coefficient increases with rising temperature $T$ and decreases with a high viscosity of the medium $\eta$ and the hydrodynamic radius of the particle $R_{H}$. This relationship is described by the Stokes-Einstein equation (equation 13) (Miller 1924).

$D=\frac{k_{B} T}{6 \pi \eta R_{H}}$

In single-particle tracking, the motion of single particles is followed over time. Temporally and spatially nearby localizations are then linked to trajectories (Figure 2.12 A). The trajectories are analyzed by calculating mean square displacements (MSD) for different time intervals $\Delta \mathrm{t}$ (equation 14). The MSD plot reports in addition to the velocity of the particles on the type of motion (Figure 2.12 B) (Michalet 2010; Renner et al. 2017).

$\operatorname{MSD}(\Delta t)=\left\langle\left(x_{i+\Delta t}-x_{i}\right)^{2}+\left(y_{i+\Delta t}-y_{i}\right)^{2}\right\rangle$

$x_{i}$ and $y_{i}$ depict the coordinates of the particle and $x_{i+\Delta t}, y_{i+\Delta t}$ represent the coordinates of the same particle after a time interval $\Delta t$. The slope of the MSD curve of a freely diffusing particle is proportional to the diffusion coefficient $D$ (equation 15, two dimensions).

$\operatorname{MSD}(\Delta t)=4 D \Delta t$

The curvature of the MSD plot reports on directed diffusion, free diffusion, confined diffusion or immobile particles (Figure $2.12 \mathrm{~B}$ ). The slope of immobile particles is typically close to zero and directed motion, e.g. transport mechanisms, show an increasing slope with increasing time intervals $\Delta t$. A linear plot is observed for freely diffusing particles exhibiting Brownian motion. Confined particles, e.g. membrane 
proteins interacting with cellular structures such as the cytoskeleton, typically show a saturation curve (Figure 2.12 B) (Michalet 2010; Rossier et al. 2012; Manzo und GarciaParajo 2015).

A

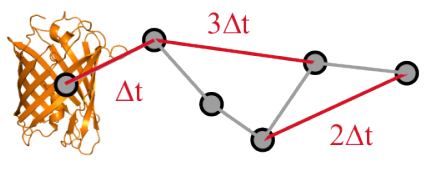

B

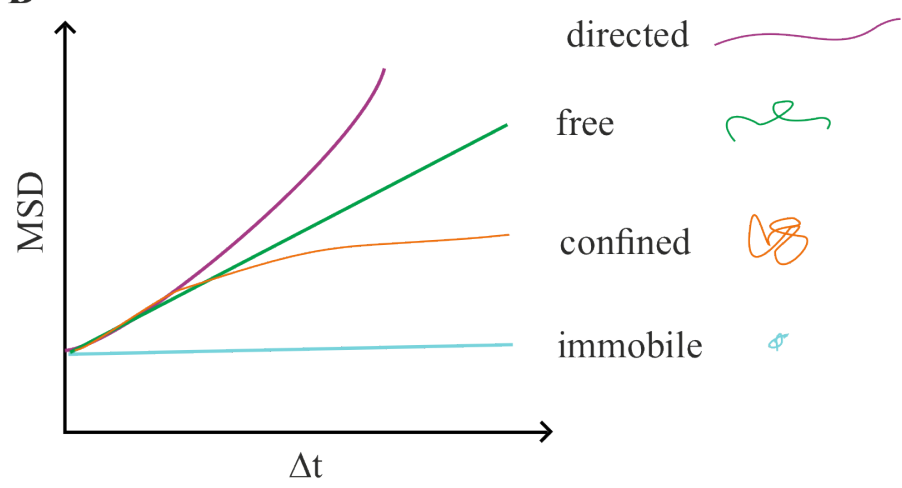

Figure 2.12: Different diffusion types show distinct behaviors in the MSD plot. (A) Localizations (grey) of a single fluorophore (orange) are determined over time and connected to trajectories. By analyzing the distances between those localizations at varying time intervals $(\Delta t)$ allows the calculation of the mean square displacements. (B) The MSD is plotted against the time interval $\Delta t$ for different types of motion. An increasing slope is characteristic for directed motion (purple). Freely diffusing particles exhibiting Brownian motion show a constant slope in the MSD plot (green). A saturation curve is observed for confined diffusing particles (orange) and immobile particles show a slope near zero (blue).

In order to distinguish different diffusion types, the mean square displacements are calculated for all trajectories. A diffusion coefficient is assigned to each trajectory according to the slope of the first four MSD values. A trajectory possessing a diffusion coefficient lower than a threshold is assigned as immobile particle. This threshold, the minimal measurable diffusion coefficient $\left(D_{\min }\right)$, is calculated with the exposure time $\left(t_{\text {frame }}\right)$ and the y-intercept of the MSD plot $(M S D(0))$, which represents the dynamic localization precision (equation 16):

$D_{\text {min }}=\frac{\left(\sqrt{\left.\frac{M S D(0)+\frac{4}{3} D \cdot t_{\text {frame }}}{4}\right)^{2}}\right.}{4 \cdot 4 \cdot t_{\text {frame }}}$ 
The MSD plot of confined diffusing particles is described by equation 17, displaying a confinement radius $\left(r_{\text {conf }}\right)$ and its diffusion coefficient $\left(D_{\text {conf }}\right)$.

$M S D(\Delta t)=\frac{4}{3} r_{\text {conf }}^{2} \cdot\left(1-e^{\frac{-\Delta t \cdot 3 D_{\text {conf }}}{r_{\text {conf }}^{2}}}\right)$

Low emitter densities are required for single-particle tracking to precisely determine the localization of the fluorophore and at the same time to allow an exact assignment of the localizations to the individual fluorophores. Single-particle tracking PALM (sptPALM) regulates the emitter density by adjusting the laser power of violet light (Manley et al. 2008). A big advantage of this technique is that the target protein is directly expressed with the fluorescent protein enabling the analysis of intracellular proteins without disturbing the cellular membrane. An alternative approach, limited to the observation of membrane proteins, is universal PAINT (UPAINT) (Giannone et al. 2010). In uPAINT, ligands are labeled with organic fluorophores and bind to their target proteins, for instance receptors (see chapter 2.3, figure $2.5 \mathrm{~B}$ ). By adjusting the ligand concentration, trajectories of individual ligand-receptor complexes are obtained. A big advantage is the high brightness and photostability of organic fluorophores allowing the observation of longer trajectories compared to sptPALM. The combination of UPAINT and sptPALM is very powerful because fluorescent proteins and red organic fluorophores are spectrally separated, allowing two-color SPT experiments of two target proteins. 


\section{Materials \& Methods}

The following chapter describes the materials and methods that were used to generate the results presented in chapter 4 . In addition, analysis parameters are described.

\subsection{Generation of plasmids}

Cloning with standard restriction enzymes was used to generate pRsetA plasmids containing mEos3.2, mEos4b or mMaple3. The DNA sequence of different fluorescent proteins were amplified with the respective primers (Table 3.1) by PCR (Bio-Rad C1000 Touch $^{\mathrm{TM}}$ Thermal Cycler, Bio-Rad laboratories, Hercules, CA, USA) and incubated with the appropriate restriction enzymes for about 1 hour. Vectors were incubated with the restriction enzymes and dephosphorylated by a thermosensitive alkaline phosphatase (FastAP, Thermo Fisher Scientific GmbH, MA, USA). The vector and the amplified insert were applied on an agarose gel, bands were extracted and purified with a PCR purification kit (Thermo Fisher Scientific $\mathrm{GmbH}$ ). Vector and insert were ligated in ratios of 1:1 to 1:5 (vector:insert) with T4 DNA ligase (Thermo Fisher Scientific GmbH) and electroporated (see section 3.2) in E.coli Top 10 cells. Single clones were grown overnight in $\mathrm{LB}$ medium containing a suitable antibiotic at $37^{\circ} \mathrm{C}$ and $200 \mathrm{rpm}$ and their plasmid DNA was purified by the GeneJET Plasmid Miniprep Kit (Thermo Fisher Scientific $\mathrm{GmbH}$ ). Finally, the DNA was sequenced (Eurofins genomics, Ebersberg, Germany). Plasmids generated are summarized in table 3.2. Plasmids of Dendra2 and PAmCherry were produced by former lab members.

Table 3.1: List of primers for cloning

\begin{tabular}{cr}
\hline \multicolumn{1}{c}{ Primer name } & Primer sequence \\
\hline mEos3.2inpRSET_fwres & GAA GGATCC ATGAGTGCGATTAAGCCAGAC \\
mEos3.2inpRSET_revres & GAA GAATTC TTA TCTTCGTCTGGCATTGTCAG \\
fw_Insert_LinkmEos3.2 & TCTGGATTGCCTGACAATGCCAGACGAAGA \\
rev_Insert_LinkmEos3.2 & ggaggtggaccggtgcccagtgggagggat \\
fw_pRset_Dimer & TTAGCAGCCGGATCAAGCTTCGAATTCTTA \\
GGATTGCCTGACAATGCCAGACGAAGATAAGAATTC \\
rev_pRset_Dimer & GAAGCTTGATCCGGCTGCTAACAA \\
BamHI_mEos4b_fw & atccctccactggggcaccggtccacctcc \\
EcoRI_mEos4b_rev & TCTTCGTCTGGCATTGTCAGGCAATCCAGA \\
\hline
\end{tabular}




\begin{tabular}{|c|c|}
\hline fw_mMaple3_BamHI_2 & ATA GGATCC atgtctggtggeggc \\
\hline rev_mMaple3_EcoRI_2 & GAC GAATTC ttatttgtacagctcatccatg \\
\hline$\overline{\mathrm{mE}} \mathrm{E} 3.2 \mathrm{fw} \mathrm{HOT}$ & $\begin{array}{l}\text { getgegctgctggcaaccecggtcgetact } \\
\text { ATGAGTGCGATTAAGCCAGACATGAAGATC }\end{array}$ \\
\hline mEos3.2_revHOT & $\begin{array}{c}\text { Cacactggatcagttatctatgcggcegcttta } \\
\text { TCTTCGTCTGGCATTGTCAGGCAATCCAGA }\end{array}$ \\
\hline CD86_fwHOT & $\begin{array}{l}\text { TCTGGATTGCCTGACAATGCCAGACGAAGATAA } \\
\text { agcggecgcatagataactgatccagtgtg }\end{array}$ \\
\hline CD86_revHOT & $\begin{array}{c}\text { GATCTTCATGTCTGGCTTAATCGCACTCAT } \\
\text { agtagcgaccggggttgccagcagcgcagc }\end{array}$ \\
\hline mEos4b_fwHOT & $\begin{array}{l}\text { getgegctgctggcaaccecggtcgetact } \\
\text { gtgagtgcgattaagccagacatgaggatc }\end{array}$ \\
\hline mEos 4 b_revHOT & cacactggatcagttatctatgcggecget tcatcgtctggcattgtcaggcaatccaga \\
\hline CD86_Eos4b_fwHOT & $\begin{array}{l}\text { tctggattgcctgacaatgccagacgatga } \\
\text { agcggecgcatagataactgatccagtgtg }\end{array}$ \\
\hline CD86_Eos4b_revHOT & $\begin{array}{l}\text { gatcctcatgtctggcttaatcgcactcac } \\
\text { agtagcgaccggggttgccagcagcgcagc }\end{array}$ \\
\hline
\end{tabular}

The plasmids encoding dimeric mEos3.2, CD86-mEos3.2, CD86-mEos4b, CTLA4mEos3.2, and CTLA-4-mEos4b were generated in hot fusion reactions as described previously (Fu et al. 2014). Primers (table 3.1) were designed that contained about 30 bases overlap and were used in a PCR reaction to amplify both the vector and the insert DNA sequence. The amplified DNA sequences were applied on an agarose gel, extracted and purified with the PCR purification kit. The DNA fragments were used in a hot fusion reaction $\left(50{ }^{\circ} \mathrm{C}\right.$ for $1 \mathrm{~h}$, cooling in $0.1^{\circ} \mathrm{C}$ steps per second to $20^{\circ} \mathrm{C}$, hold at $10^{\circ} \mathrm{C}$ ) containing the preassembly buffer $(0.5 \mathrm{M}$ TRIS $\mathrm{HCl} \mathrm{pH} 7.5,50 \mathrm{mM} \mathrm{MgCl} 2,1 \mathrm{mM}$ of each dNTP, $50 \mathrm{mM}$ DTT, 25\% PEG 8000), $0.0075 \mathrm{u} / \mu \mathrm{L}$ T5 exonuclease (New England Biolabs GmbH, MA, USA) and $0.05 \mathrm{u} / \mu \mathrm{L}$ Phusion polymerase (Thermo Fisher Scientific $\mathrm{GmbH}$ ). Finally, the product of the hot fusion reaction was electroporated (see section 3.2) into E.coli Top10 cells and the plasmid DNA of single clones was purified and verified by sequencing.

Table 3.2: List of generated plasmids

\begin{tabular}{ccl}
\hline Insert & Vector & Cloning method \\
\hline mEos3.2 & pRsetA & Restriction enzyme \\
\hline mEos3.2 dimer & pRsetA & Hot fusion reaction \\
mEos4b & pRsetA & Restriction enzyme \\
mMaple3 & pRsetA & Restriction enzyme \\
mEos3.2 & pIRESpuro2- & Hot fusion reaction \\
& CD86 & \\
\hline
\end{tabular}




\begin{tabular}{ccc}
\hline mEos3.2 & pIRESpuro2- & Hot fusion reaction \\
& CTLA4 & \\
mEos4b & pIRESpuro2- & Hot fusion reaction \\
& CD86 & \\
mEos4b & pIRESpuro2- & Hot fusion reaction \\
& CTLA4 & \\
\hline
\end{tabular}

\subsection{Electroporation}

Electroporation was performed by using the BTX Harvard Apparatus Gemini System (Thermo Fisher Scientific GmbH). Approximately 2-3 $\mu \mathrm{L}$ of plasmid was added to $80 \mu \mathrm{L}$ of electrocompetent E.coli Top10 cells. The electroporation cuvettes were precooled on ice and the pulse program $(25 \mu \mathrm{F}$ and $200 \Omega)$ of standard E.coli cells was used. After electroporation, cells were incubated in prewarmed SOC medium at $37^{\circ} \mathrm{C}$ and $200 \mathrm{rpm}$ for about 1 hour. Finally, cells were transferred on agar plates containing the appropriate anitiobtic.

\subsection{Protein expression and purification in E.coli}

Plasmids encoding mEos3.2, dimeric mEos3.2, mEos4b, and mMaple3 were electroporated in E.coli BL21-DE3 cells. The next day, single clones of the electroporated cells grown on a fresh agar plate were picked and used to inoculate $10 \mathrm{~mL}$ of LB medium. After about $16 \mathrm{~h}$, the preculture was transferred into $800 \mathrm{~mL}$ LB medium with the appropriate antibiotic and grown to an $\mathrm{OD}_{600}$ of about 0.4 . Protein expression was induced by adding $1 \mathrm{mM}$ isopropyl-ß-D-thiogalactopyranosid (IPTG, Cayman chemical company, MI, USA) to the cells for $2-16 \mathrm{~h}$ at $20^{\circ} \mathrm{C}$ and $200 \mathrm{rpm}$. Cells were harvested by centrifugation at 4,000 x g for $10 \mathrm{~min}$ (Megafuge 1.0, Heraeus, Hanau, Germany). The cell pellet was directly used or stored at $-20^{\circ} \mathrm{C}$. Next, the cells were lysed in about $4 \mathrm{~mL}$ lysis buffer (50 mM NaH${ }_{2} \mathrm{PO}_{4}$ (Sigma-Aldrich, MO, USA), $300 \mathrm{mM} \mathrm{NaCl}$ (SigmaAldrich), $10 \mathrm{mM}$ imidazole (Sigma-Aldrich)) containing $1 \mathrm{mM}$ lysozyme (SigmaAldrich) and half of a cOmplete Mini EDTA-free protease inhibitor tablet (Roche, Basel, Switzerland). Cell lysis was supported by 4 x 1 min cycles of sonification (Sonifier 250, Branson Ultrasonics, CT, USA). The solubilized cells were then centrifuged two times at $16,900 \times \mathrm{g}$ for $15 \mathrm{~min}$ (Centrifuge $5418 \mathrm{R}$, Eppendorf, Hamburg, Germany) and the supernatant containing the respective His-tagged protein was applied onto an equilibrated Ni-NTA (Qiagen, Venlo, Netherlands) column. The column was washed with lysis buffer 
containing $20 \mathrm{mM}$ imidazole and the protein was eluted with lysis buffer containing 250 $\mathrm{mM}$ imidazole. PD10 desalting size-exclusion columns (Bio-Rad, CA, USA) were used to exchange the buffer to phosphate buffered saline (1xDPBS, Gibco by Thermo Fisher Scientific). The purified protein was concentrated and applied on a SDS-PAGE gel (4$20 \%$ gradient SDS gels, BioRad Laboratories, Hercules, USA) to verify the correct size of the protein.

\subsection{Generation of a 30-base pair DNA-linker for dimerization of His-tagged mEos3.2}

The generation of a 30-base pair DNA-linker was performed by Karl Gatterdam in the lab of Prof. Robert Tampé (Experimental details are published in (Baldering et al. 2019b).

\subsection{Cell lines}

Cell lines were cultivated in Dulbecco's modified Eagle medium (DMEM) (Gibco, LifeTechnologies) supplemented with 1\% GlutaMAX (Gibco, Life Technologies) and $10 \%$ fetal bovine serum (Gibco, Life Technologies) in an $\mathrm{CO}_{2}$ incubator (Model C 150; Binder $\mathrm{GmbH}$, Tuttlingen, Germany) at $37{ }^{\circ} \mathrm{C}$ and $5 \% \mathrm{CO}_{2}$. Cells were passaged every third or fourth day via trypsinization. Cell lines that were used in this thesis are listed in table 3.3.

Table 3.3: List of cell lines

\begin{tabular}{ll}
\hline Cell line & Description/Company \\
\hline HeLa cells & Institut für angewandte Zellkultur, Munich, Germany \\
HEK293T & A kind gift of Prof. Hartmut Niemann (University of Bielefeld) \\
MET C6 & A stable MET-mEos4b cell line generated by CRISPR/Cas12a- \\
& assisted PCR tagging \\
EGFR F8 & A stable EGFR-mEos4b cell line generated by CRISPR/Cas12a- \\
& assisted PCR tagging \\
\hline
\end{tabular}

\subsection{Generation of PCR cassettes for CRISPR/Cas12a-assisted PCR tagging}

The CRISPR/Cas12a system was used for endogenous tagging of MET and EGFR. The generation of the respective PCR cassettes was performed as previously described (Füller et al 2020). Briefly, primer M1 and M2 (table 3.4) were designed with the help of a web interface (www.pcr-tagging.com) and used to produce the PCR cassettes. The PCR fragments were applied on an agarose gel and the bands corresponding to the PCR cassettes were extracted, purified and further used to transfect HEK293T cells. 
Table 3.4: List of primers for CRISPR/Cas12a-assisted PCR tagging. DNA bases with stars $\left(^{*}\right)$ indicate phosphorothioate bond modifications.

\begin{tabular}{|c|c|}
\hline $\begin{array}{l}\text { Primer } \\
\text { name }\end{array}$ & Sequence \\
\hline M1_MET & $\begin{array}{c}\mathrm{T}^{*} \mathrm{G} * \mathrm{~T}^{*} \mathrm{G} * \mathrm{~T}^{*} \text { CGCTCCGTATCCTTCTCTGTTGTCATCAGAAGATAAC } \\
\text { GCTGATGATGAGGTGGACACACGACCAGCCTCCTTCTGGGAGACA } \\
\text { TCATCAGGTGGAGGAGGTAGTG }\end{array}$ \\
\hline M2_MET & $\begin{array}{c}C * A * G * T * G * A A A A A A C C A T T G G A C A A A G T G T G G A C T G T T G C T T T G \\
\text { ACATAGTACTAGCAAAAAAAACTAGCACTATGATGTCTCCATCTA } \\
\text { CACTTAGTAGAAATTAGCT AGCTGCATCGGTACC }\end{array}$ \\
\hline M1_EGFR & $\begin{array}{c}\mathrm{G} * \mathrm{C}^{*} \mathrm{C} * \mathrm{~A} * \mathrm{~A} * \mathrm{GCCAAATGGCATCTTTAAGGGCTCCACAGCTGAAAA} \\
\text { TGCAGAATACCTAAGGGTCGCGCCACAAAGCAGTGAATTTATTGG } \\
\text { AGCATCAGGTGGAGGAGGTAGTG }\end{array}$ \\
\hline M2_EGFR & $\begin{array}{l}\mathrm{T}^{*} \mathrm{G} * \mathrm{G}^{*} \mathrm{~T} * \mathrm{C}^{*} \text { CTGGGTATCGAAAGAGTCTGGATTTTTAGGGCTCATA } \\
\text { CTATCCTCCGTGGAAAAAACCTCCGTGGTCATGCTCCAAATCTACA } \\
\text { CTTAGTAGAAATTAGCTA GCTGCATCGGTACC }\end{array}$ \\
\hline
\end{tabular}

\subsection{Transfection}

Cells were seeded on 6-well or 24-well plates at densities of $20-30 \cdot 10^{4}$ cells/well or $4 \cdot 10^{4}$ cells/well, respectively. After about $24 \mathrm{~h}$, cells were transfected with the desired plasmids or/and DNA fragments by the use of Lipofectamine 3000 (Thermo Fisher Scientific) according to the manufacturer instructions.

\subsection{Generation of stable cell lines}

Transfected cells were incubated in a $\mathrm{CO}_{2}$ incubator (Model C 150; Binder $\mathrm{GmbH}$, Tuttlingen, Germany) at $37{ }^{\circ} \mathrm{C}$ and $5 \% \mathrm{CO}_{2}$ for approximately $72 \mathrm{~h}$. Cells were transferred into 6-well plates and puromycin was added at a concentration of about $1.5 \mu \mathrm{g} / \mathrm{mL}$ to select the positive CRISPR/Cas12a-edited cells via antibiotic resistance. Cells were further cultivated for approximately 1-2 weeks to reach a density that was suitable for transferring the cells into a 775 flask (Greiner Bio-One international GmbH). 96-well plates (Thermo Fisher Scientific) containing 1 cell/well were produced by limited dilution. After about one to two weeks, single wells were analyzed with regard to the number of single clones and wells containing cells from solely one clone were transferred into one well in a 24 well-plate. Cells were further cultivated for genome isolation, western blotting and analysis through fluorescence microscopy. 


\subsection{Isolation and analysis of genomic DNA}

The Genomic DNA Purification Kit (Thermo Fisher Scientific GmbH) was used for genome isolation. After determining the concentration of the genomic DNA, a PCR was performed with primers (table 3.5) annealing in- and outside of the PCR cassette. PCR fragments were applied on an agarose gel and purified by the PCR purification kit. Positive clones were verified by sequence analysis.

Table 3.5: List of primers for genome analysis

\begin{tabular}{cc}
\hline Primer name & Sequence \\
\hline gMET_ORF_fw & GAGCTGATGACAAGAGGAGC \\
mEos4b_rev & CCCTTCTCCTTAGCTTTGTAAG \\
mEos4b_fw & GAAAATGTATGTGCGTGATGG \\
gMET_UTR_rev & CTGCTTCAATTTCCCATATGAAA \\
gEGFR_ORF_fw & CATTCACAGGGTTCAGAACCCAG \\
gEGFR_UTR_rev & GATCCCCAATCAATAAAAATCCTCAC \\
\hline
\end{tabular}

\subsection{Western blot}

About $1 \cdot 10^{6}$ cells were seeded on $10 \mathrm{~cm}$ dishes and cultivated for 24-72 h. Cell medium was changed to serum-free medium approximately $12-16 \mathrm{~h}$ prior to stimulating the cells with $1 \mathrm{nM}$ human HGF (PeproTech GmbH, Hamburg, Germany), $1 \mathrm{nM}$ InlB 321 -ATTO $647 \mathrm{~N}, 16 \mathrm{nM}$ EGF (Sigma-Aldrich) or $16 \mathrm{nM} \mathrm{EGF-CF640} \mathrm{for} 10 \mathrm{~min}$ at $37^{\circ} \mathrm{C}$. Cells were harvested with $200 \mu \mathrm{L}$ of lysis buffer (50 mM TRIS (Sigma-Aldrich), $150 \mathrm{mM} \mathrm{NaCl}$ (Sigma-Aldrich), 1\% Triton X-100 (Sigma-Aldrich), 1 mM Na $\mathrm{VO}_{4}$ (Sigma-Aldrich), $1 \mathrm{mM}$ EDTA (Sigma-Aldrich), $1 \mathrm{mM}$ NaF (Sigma-Aldrich), and one-fourth of a cOmplete Mini EDTA-free protease inhibitor tablet (Roche)) and by scraping the cells from the dish. After incubating the cells in a thermo shaker (Thermo-Shaker, Universal Labortechnik GmbH \& Co. KG, Leipzig, Germany) at $750 \mathrm{rpm}, 4{ }^{\circ} \mathrm{C}$ for $5 \mathrm{~min}$, cells were centrifuged at $12,000 \mathrm{x} \mathrm{g}, 4^{\circ} \mathrm{C}$ for $20 \mathrm{~min}$ (Centrifuge $5418 \mathrm{R}$, Eppendorf). The supernatant was supplemented with 5x SDS-loading dye (250 mM TRIS HCl (pH 6.8), 8\% (w/v) SDS (Sigma-Aldrich), 0.1\% bromphenol blue (Sigma-Aldrich), 40\% (v/v) glycerol (Roth)) and $100 \mathrm{mM}$ dithiothreitol (DTT, Sigma-Aldrich) and applied on a 4$20 \%$ gradient SDS gel (BioRad Laboratories). SDS-PAGE was performed at $200 \mathrm{~V}$ for 30-90 min. Protein bands were blotted on a membrane with the iBlot Gel Transfer System (Thermo Fisher Scientific) as described by the manufacturer. Then, the membrane was 
blocked with 5\% (w/v) nonfat dry milk (Cell Signaling Technology, MA, USA) in TRISbuffered saline containing $0.1 \%$ of Triton X-100 (TBST) at room temperature for 1 hour. After washing the membrane three times with $10 \mathrm{~mL}$ TBST, the membrane was incubated with a primary antibody (MET antibody, \#4560; phospho-MET antibody (tyr1234/1235), \#3077; phospho-MAPK antibody, \#9101S; Cell SignalingTechnology, MA, USA, $1: 1000)$ at $4{ }^{\circ} \mathrm{C}$ overnight. Then, three washing steps with $10 \mathrm{~mL}$ TBST were performed and the secondary HRP-tagged antibody (goat anti-rabbit IgG, Jackson ImmunoResearch, PA, USA, 1:20,000) was added at room temperature for 3 hours. The membrane was again washed three times with $10 \mathrm{~mL}$ TBST and once with $10 \mathrm{~mL}$ TBS at room temperature for 5-15 min. Finally, the desired bands were detected with a Fusion FX Edge imager (Vilber Lourmat, Collégien, France) by using $1 \mathrm{~mL}$ of SuperSignal West Femto Maximum Sensitivity Substrate solution (Thermo Fisher Scientific). The intensity of the protein bands was analyzed with Fiji (Schindelin et al. 2012).

\subsection{Preparation of functionalized glass slides for SMLM}

Round cover glasses (diameter $25 \mathrm{~mm}$, VWR International, Radnor, USA) or square cover glasses $(35 \times 64 \mathrm{~mm}$, \# 1.5, Thermo Fisher Scientific) were incubated in isopropanol (VWR Chemicals) for $20 \mathrm{~min}$ and plasmacleaned with oxygen or nitrogen for $15 \mathrm{~min}$ (Diener Electronic GmbH, Ebhausen, Germany). After that, cover glasses were coated with $100 \mu \mathrm{g} / \mathrm{mL}$ poly-L-lysine (Sigma-Aldrich) or $0.8 \mathrm{mg} / \mathrm{mL}$ PLL-PEG-RGD (for details see (Harwardt et al. 2020)) in PBS for 1.5-2 hours and washed with distilled water. Cover glasses were dried with nitrogen, and used immediately or stored under argon atmosphere at $-20^{\circ} \mathrm{C}$.

\subsection{Fluorescence microscopes}

Two fluorescence microscopes were used in this thesis. Quantitative PALM experiments were primarily performed on a home-built setup (Figure 3.1). The setup is equipped with an inverted microscope (IX-71, Olympus), a $405 \mathrm{~nm}$ laser (LBX-405-50-CSB-PP, Oxxius, Germany), a $568 \mathrm{~nm}$ laser (Sapphire 568 LP, Coherent, Germany), and a $643 \mathrm{~nm}$ laser (iBEAM smart, Toptica, Germany). The 568 and $643 \mathrm{~nm}$ lasers are combined with dichroic mirrors and pass an acousto-optic tunable filter (AOTF, AAOptics) for filtering and intensity adjustment. The laser power of the $405 \mathrm{~nm}$ laser is adjusted by a neutral density filter wheel (Thorlabs) and aligned with the other laser lines. All laser lines are expanded and focused on the back focal plane of the objective by a telescope. Different 
illumination schemes can be selected by adjusting the position of a movable mirror. A dichroic mirror (HC Quad 410/504/582/669, AHF, Germany) directs the excitation light through a 100x oil immersion objective (PLAPO 100x TIRFM, NA $\geq 1.45$, Olympus, Germany) onto the sample. To minimize axial drift, the microscope is equipped with a nosepiece. The fluorescence light of the sample is collected by the same objective and passes the dichroic mirror. An Optosplit II (Cairn Research, United Kingdom) enables two channel imaging. The emission light is filtered by appropriate bandpass filters (BrightLine HC 590/20 or ET 700/75, AHF Analysentechnik, Germany) and detected by an EMCCD camera (iXon Ultra X-10971, Andor Technology, UK) with an image pixel size of $157 \mathrm{~nm}$.

A commercial N-STORM microscope (Nikon, Germany) was used for some qPALM experiments as well as the sptPALM and uPAINT experiments. This microscope is equipped with 405, 561 and $647 \mathrm{~nm}$ lasers, an 100x objective (Apo TIRF oil, 1.49 NA) and was operated in TIRF mode. The emission light is collected by an EMCCD camera (DU-897U-CS0-BV, Andor Technology, UK) with an image pixel size of $158 \mathrm{~nm}$.

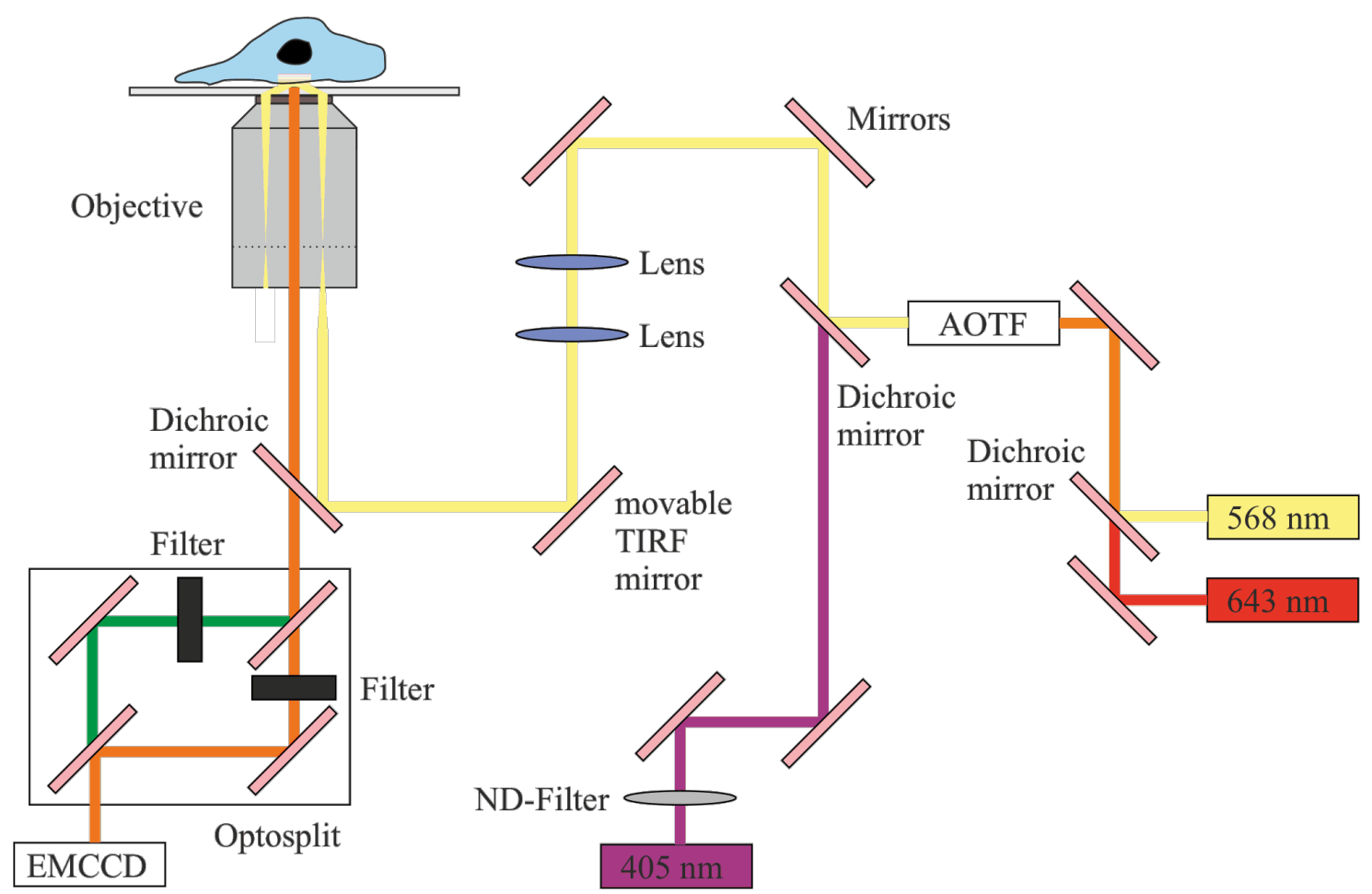

Figure 3.1: Home-built microscope setup. Lasers $(568 \mathrm{~nm}, 643 \mathrm{~nm})$ are aligned and coupled into an acousto optical tunable filter (AOTF) and aligned with a $405 \mathrm{~nm}$ laser. A telescope consisting of two lenses expands the laser beams and focuses the beam on the back focal plane of the objective. A movable TIRF mirror enables to switch between widefield and TIRF illumination schemes. Fluorescence is collected by the same objective. The emission fluorescence (orange) 42 
passes an optosplit, which allows for two channel imaging and filtering of distinct wavelengths. Finally, the emitted photons are detected by an EMCCD camera.

\subsection{Sample preparation for qPALM measurements in vitro}

flexiPERM chambers (Sarstedt) were placed on square cover glasses functionalized with poly-L-lysine (Sigma-Aldrich). The purified fluorescent proteins were immobilized at varying concentrations from $500 \mathrm{pM}$ to $2 \mathrm{nM}$ and incubated for $30 \mathrm{~min}$ at room temperature. The chambers were washed three times with sterile-filtered PBS and fluorescent proteins were imaged in sterile-filtered PBS or sterile-filtered PBS supplemented with $100 \mathrm{mM}$ MEA at $\mathrm{pH} \sim 7.8$.

\subsection{Sample preparation for qPALM measurements in cellulo}

flexiPERM chambers (Sarstedt, Germany) were placed on square cover glasses functionalized with PLL-PEG-RGD. Cells of stable cell lines (MET C6; EGFR F8) or transfected cells in 6-well plates were detached with PBS by careful resuspension and transferred into flexiPERM chambers at densities of $\sim 2 \cdot 10^{4}$ cells/well. After about 16 $\mathrm{h}$ incubation at $37{ }^{\circ} \mathrm{C}$ with $5 \% \mathrm{CO}_{2}$, cells were treated with ligands or directly fixed. For ligand treatment, cells were incubated in serum-free DMEM for about 4 hours, incubated 5 min at $4^{\circ} \mathrm{C}$ and then ligands (HGF, $1 \mathrm{nM}$; InlB 321 -ATTO 647N, 1 or $5 \mathrm{nM}$; EGF, 16 $\mathrm{nM}$ ) were added in serum-free DMEM for $10 \mathrm{~min}$ at $4^{\circ} \mathrm{C}$. After that, cells were washed with $400 \mathrm{mM}$ saccharose in PBS and then directly fixed with $4 \%$ formaldehyde (Thermofisher Scientific, Germany), 0.2\% glutaraldehyde (Merck, Germany) and 400 $\mathrm{mM}$ saccharose in PBS for a minimum of $15 \mathrm{~min}$ at $4^{\circ} \mathrm{C}$. Cells were then washed three times with sterile-filtered PBS and imaged in sterile-filtered PBS.

\subsection{5 qPALM imaging}

The home-built microscope was used with laser powers of $0-30 \mathrm{~mW} / \mathrm{cm}^{2}(405 \mathrm{~nm}), 0.21$ $\mathrm{kW} / \mathrm{cm}^{2}(568 \mathrm{~nm})$, and $4.6 \mathrm{~W} / \mathrm{cm}^{2}(643 \mathrm{~nm})$ and videos were recorded with TIR illumination. SMLM videos had a length of 10,000 - 80,000 frames with an exposure time of $100 \mathrm{~ms}$. For amplification of the signal, an EM gain of 200 and a preamplifier gain of 1 was used.

The commercial microscope (N-STORM, Nikon) was used with laser powers of 0-38 $\mathrm{mW} / \mathrm{cm}^{2}(405 \mathrm{~nm})$ and $0.2 \mathrm{~kW} / \mathrm{cm}^{2}(568 \mathrm{~nm})$ and videos were recorded with TIR illumination. SMLM videos had a length of 18,000 - 54,000 frames with an exposure 
time of $100 \mathrm{~ms}$. For amplification of the signal, an EM gain of 200 and a preamplifier gain of 1 was used. Image acquisition was controlled by $\mu$-Manager (version 1.4.20) (Edelstein et al. 2014) and NIS-Elements.

\subsection{6 qPALM analysis}

Fluorescence signals in the SMLM videos were localized using an open source software, rapidSTORM (v.3.3) (Wolter et al. 2010), with a pixel size of $157 \mathrm{~nm}$, a PSF FWHM of $360 \mathrm{~nm}$ and an intensity threshold of 63 photons (intensity threshold in rapidSTORM: 1021). A super-resolved image was generated by adjusting the intensity cutoff to a maximum of $3.28 \mathrm{k}$ ADC. The Kalman filtering tool was used to combine fluorescence events that occurred in consecutive frames into a single localization. A post-processing software, LocAlization Microscopy Analyzer (LAMA) (Malkusch and Heilemann 2016), was then used to analyze the number of tracked localizations within each localization cluster. The individual localization clusters are selected in Fiji and the respective numbers of blinking events are extracted. The relative frequency of the number of blinking events of at least 500 cluster of three independent experiments was then plotted and fitted by hypergeometric functions with the respective parameters (equations 10-12) with OriginPro 2017G (v9.40, OriginLab). For the results in chapters 4.2 and 4.3, a qSMLM software (Baldering et al. 2019a) was used for analysis of the data. At least 10 cells were analyzed for each imaging condition.

\subsection{Sample preparation for sptPALM and UPAINT measurements}

Cells were seeded on round cover glasses coated with PLL-PEG-RGD at a density of $\sim 25$ $\cdot 10^{4}$ cells/well. After approximately $16-24 \mathrm{~h}$, the cover glasses were transferred into home-built holders. The cells were washed once with serum-free imaging medium containing DMEM with 1\% GlutaMAX and 50 mM HEPES buffer (pH 7.2-7.5; Gibco, Life Technologies). HGF or InlB $321-A T T O 647 \mathrm{~N}$ was added to the cells at concentrations of $1 \mathrm{nM}$, respectively.

\subsection{8 sptPALM and UPAINT imaging}

Single-particle tracking was performed using the commercial N-STORM microscope with laser powers of 0-38 mW/ $\mathrm{cm}^{2}(405 \mathrm{~nm})$ and $0.2 \mathrm{~kW} / \mathrm{cm}^{2}(561 \mathrm{~nm})$, and $0.2 \mathrm{~kW} / \mathrm{cm}^{2}$ $(647 \mathrm{~nm})$ and in TIR mode. Image acquisition was controlled by $\mu$-Manager (version 
1.4.20) (Edelstein et al. 2014) and NIS-Elements using an exposure time of $20 \mathrm{~ms}$ and an EM gain of 300. Videos had a length of 1,000 frames.

\subsection{9 sptPALM and UPAINT analysis}

SMLM videos were analyzed with PALM-Tracer (Bordeaux Imaging Center), a plugin for MetaMorph (Molecular Devices, USA). Analysis parameters and a detailed procedure is described in Harwardt et al. 2017. Briefly, PALM-Tracer localizes single molecules by centroid fitting, connects localizations that are less than $790 \mathrm{~nm}$ apart (5 pixels), and yields a diffusion coefficient from the MSD plot of each trajectory. Diffusion coefficients were determined from fitting the first four points of the MSD plot. Mean diffusion coefficients were calculated in OriginPro $2017 \mathrm{G}$ by averaging over at least 20 cells per imaging condition. In addition, each trajectory was assigned to immobile, confined or free motion as described previously (Rossier et al. 2012). The minimal measurable diffusion coefficient $D_{\min }$ was calculated by equation 16 with $M S D(0)$ as dynamic localization uncertainty (Michalet 2010) and used for all imaging conditions.

Every trajectory that exhibits a diffusion coefficient smaller than the $D_{\min }$ value is assigned as immobile. Confined and free particles were further differentiated according to Rossier et al. 2012. The relative frequency of each motion type was plotted in a bar diagram with the standard error of the mean.

\subsection{Stochastic simulation of photophysics tool (ssp)}

The stochastic simulation of photophysics tool ( $\mathrm{ssp}$ ) is an open source software, freely available for download on GitHub (https://github.com/SMLMS), and was programmed by Sebastian Malkusch. The software uses Monte-Carlo simulations to simulate the number of blinking events for different types of oligomer with a defined bleaching and detection probability. The blinking kinetics is based on the four state model of fluorescent proteins described previously (Annibale et al. 2010). First, the ssp tool defines whether a molecule is detected or not by referring to the detection probability $d$. If the molecule is detected, it counts all transitions into the dark state until it reaches the bleached state based on the bleaching probability $p$. In the case of monomers only, it directly outputs the number of blinking events per particle. For higher ordered oligomers, the blinking events are summed up for all particles representing this oligomer. Finally, a probability mass function $(\mathrm{PMF})$ is generated from the blinking events of simulated oligomers, which 
3 Materials \& Methods

can then be fitted by the open source qSMLM software also available on GitHub (https://github.com/SMLMS). 


\section{Results \& Discussion}

One benefit of fluorescence microscopy is the opportunity to quantify protein molecules directly in cells. In single-molecule fluorescence microscopy, several approaches were developed in the past. Various approaches have been suggested for the quantitative analysis of single-molecule data (Gordon et al. 2004; Sugiyama et al. 2005; Mutch et al. 2007). At low protein densities, photobleaching experiments yield the number of bleaching steps and report on the number of molecules in protein assemblies (Leake et al. 2006; Ulbrich und Isacoff 2007; Penna et al. 2008; Ji et al. 2008; Liesche et al. 2015). If the single-molecule emitters are closer than the diffraction limit, photobleaching analysis may lead to inaccurate results (Teramura et al. 2006). Here, the quantitative analysis of single-molecule localization data offers a solution. One branch of techniques analyses the kinetics of single-molecule "blinking" (Sengupta et al. 2011; Lee et al. 2012; Puchner et al. 2013; Hummer et al. 2016; Nasu et al. 2016; Jungmann et al. 2016; Zanacchi et al. 2017). The distribution of the number of blinking events of single fluorophores is related to molecule numbers through kinetic equations and can inform on oligomeric states of fluorophore-labeled proteins. If fluorescent proteins are used, we term this method quantitative PALM (qPALM) (Fricke et al. 2015; Hummer et al. 2016; Karathanasis et al. 2017).

This chapter summarizes the development in theory and practical application of qPALM. In the first section, various fluorescent proteins are characterized with respect to their blinking properties and bleaching probability. The general applicability of qPALM for the determination of dimer fractions is demonstrated in vitro using synthetic and genetic dimers. Furthermore, the applicability of qPALM is demonstrated by the analysis of reference proteins in cellulo. The second section shows advancements in the theory of qPALM by implementing (i) a detection efficiency that allows the accurate analysis of higher-ordered oligomers and (ii) a correction term for undetected oligomers. In the third section, a novel method for chromosomal protein tagging is reported. qPALM is applied to a stable cell line expressing MET-mEos $4 b$. The cell line was generated by CRISPR/Cas12a-assisted PCR tagging, and the benefits of PCR tagging in combination with SMLM are further demonstrated by tracking the MET receptor in living cells. Finally, an automated analysis tool is demonstrated that outperforms manual analysis of qPALM in terms of analysis speed and accuracy. 


\subsection{Calibration references for quantitative PALM}

The determination of oligomers by qPALM requires the knowledge of two parameters, the bleaching probability $p$ and a correction for undetected molecules $q$. While the bleaching probability describes the blinking kinetics of fluorophores and thus corrects for overcounting, the $q$-value takes undetected molecules into account and hence corrects for undercounting. In this section, various fluorescent proteins are evaluated with respect to their bleaching probabilities. Furthermore, synthetic and genetic dimers were generated as reference structures for qPALM in vitro. For cells, reference protein structures, CD86mEos3.2, CD86-mEos4b, CTLA-4-mEos3.2, and CTLA-4-mEos4b, were generated and measured in HeLa or HEK293T cells. The $p$ - and $q$-value were determined from the respective measurements. Furthermore, simulations were performed to estimate the accuracy of qPALM to determine higher-ordered oligomers and mixtures of monomers and dimers. Finally, the measured $p$ - and $q$-values are discussed and compared with previous studies.

\subsubsection{Blinking characteristics of photoactivatable and -convertible fluorescent proteins}

Fluorescent proteins often show reappearance of fluorescence emission, which is termed "blinking". The photophysical cycle is described by a four-state kinetic model (Annibale et al. 2010) (Figure 4.1.1 A). Once a fluorescent protein is activated by violet light (Figure 4.1.1 B), it enters a detectable fluorescent state and emits photons. From this state, the fluorescent protein can reversibly transit into a dark state $\left(\mathrm{k}_{\text {off }} / \mathrm{k}_{\text {on }}\right)$ or photobleach irreversibly ( $\mathrm{k}_{\text {bleach }}$ ) (Figure 4.1.1 A). These photokinetics are taken into account by the $p$-value, which describes the probability of a fluorescent protein being directly photobleached after initial activation (Hummer et al. 2016). In practice, the number of blinking events $n$ is extracted from intensity-time traces of single-molecule detection events, and equals the number of emission events minus one, and hence the number of "reappearances of fluorescence" (Figure 4.1.1 C). The relative frequency distribution of the number of blinking events can finally be approximated by probability mass functions and reports on molecule numbers (Hummer et al. 2016). 
A

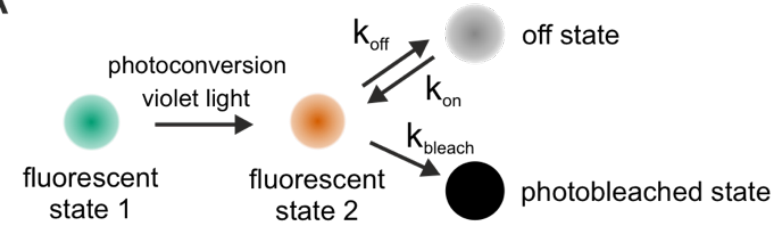

B

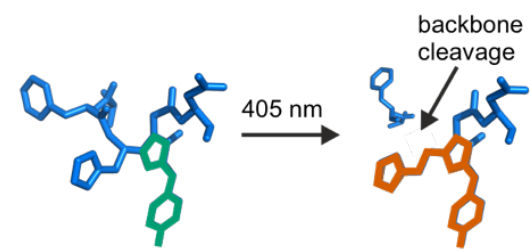

C
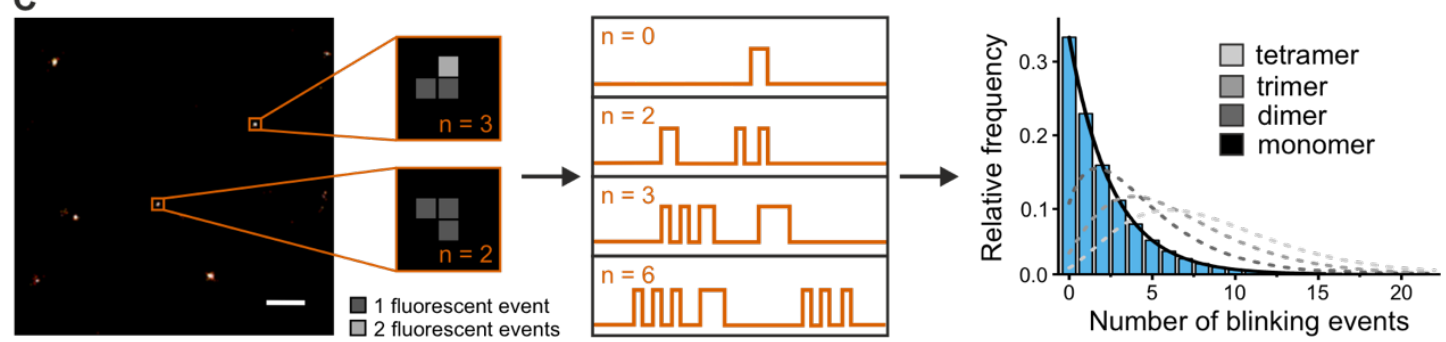

Figure 4.1.1: The workflow of qPALM. (A) 4-state stochastic model for describing the photokinetics of photoactivatable or -convertible fluorescent proteins. The fluorophore is activated or switched by violet light into the fluorescent state 2 from which it can reversibly switch into an off state or photobleach irreversibly. (B) Illustration of the backbone cleavage of mEos2 by violet irradiation extending the chromophore (orange) and leading to absorption at higher wavelengths. The 3-dimensional representation of the amino acid sequence was generated using PyMOL and the crystal structure of mEos2 (PDB: 3S05). (C) Super-resolved PALM image of single mEos3.2 molecules on a glass surface (left). Zoom-ins show the number of blinking events. Intensity-time traces are schematized (middle). The blinking distribution reports molecule numbers of single protein spots (right). Adapted from Baldering et al (2019b). Copyright CC BYNC-SA 3.0.

A selection of monomeric fluorescent proteins was adsorbed on a glass surface and imaged with qPALM. The number of blinking events was determined and plotted in histograms (Figure 4.1.2). The bleaching probabilities ( $p$-values) of four fluorescent proteins were determined. For mEos3.2, a high number of blinking events were found resulting in a small $p$-value of 0.32 . A similar $p$-value was obtained for mMaple3 ( $p=$ 0.28). Dendra 2 showed fewer blinking events and therefore a higher $p$-value $(p=0.54)$. The highest $p$-value was determined for PAmCherry2 $(p=0.77)$, with the majority of fluorophores being directly photobleached after initial activation.
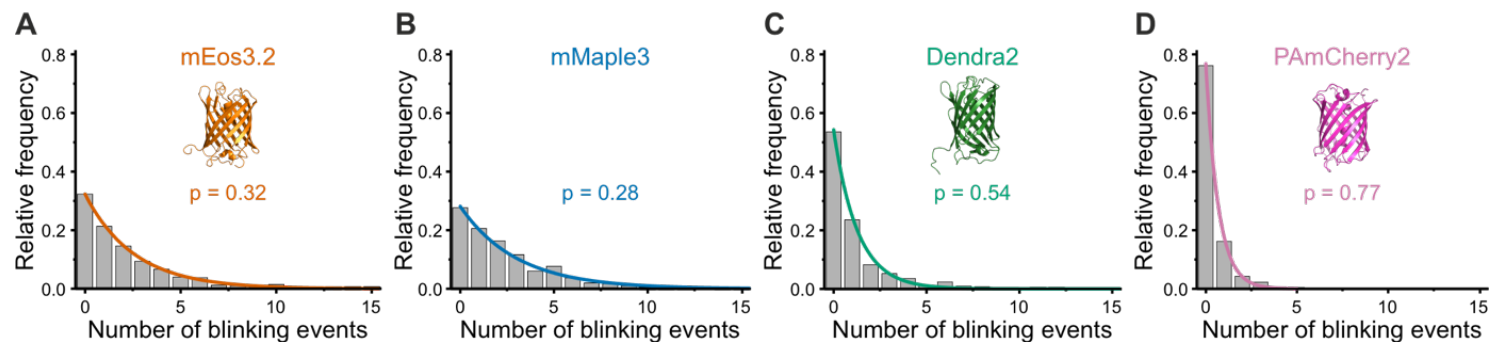
Figure 4.1.2: Blinking properties of four fluorescent proteins determined by qPALM. Histograms of the relative frequencies of the number of blinking events were fitted with equation 11 to obtain the $p$-values for mEos3.2 (A), mMaple3 (B), Dendra2 (C), and PAmCherry2 (D). Crystal structures of mEos2 (PDB: 3S05, A), Dendra2 (PDB:2VZX, C), and PAmCherry1 (PDB: 3KCT, D) are shown. Adapted from (Baldering et al 2019b). Copyright CC BY-NC-SA 3.0.

Next, $p$-values of fluorescent protein variants from the Eos family were measured and analyzed. The fluorescent protein mEos $4 b$ is the most recently developed variant of the Eos family and was developed to be suitable in combination with electron microscopy (Paez-Segala et al. 2015). The qPALM analysis of mEos $4 b$ revealed a $p$-value of 0.34 , which is very similar to the $p$-value of $\operatorname{mEos} 3.2(p=0.32$ ) (Figure 4.1.2 A, 4.1.3 A). In addition, the dependency of the $p$-value on the microscope setup was assessed. qPALM imaging on a commercial microscope (N-STORM) with similar laser intensities revealed a $p$-value of 0.30 , which differs by $7 \%$ from the $p$-value on the home-built microscope (Figure 4.1.3 B) (see section 3.14 for a detailed description of the microscope setups).

A

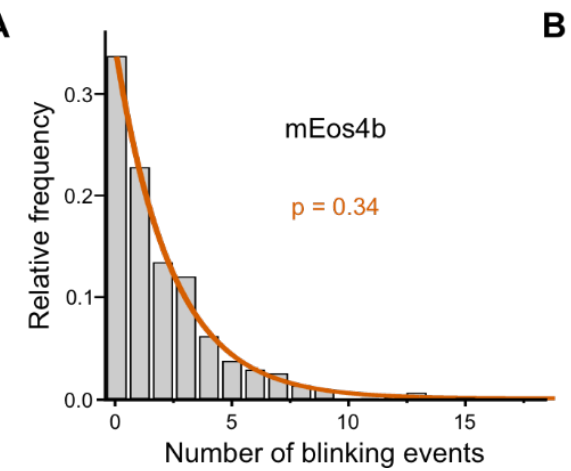

B

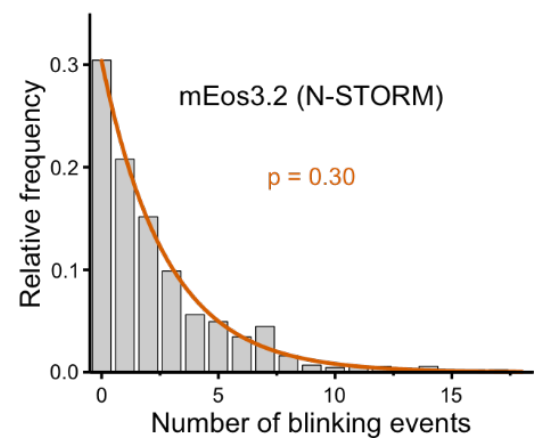

Figure 4.1.3: Blinking properties of mEos4b and mEos3.2. Histograms of the relative frequencies of the number of blinking events for different variants of the Eos family fitted by equation 11 to obtain the $p$-value for mEos $4 b(\mathrm{~A})$ and mEos3.2 measured on a N-STORM microscope (B). Adapted from Baldering et al (2019b). Copyright CC BY-NC-SA 3.0.

It was shown that blinking is responsive to the redox properties of the imaging buffer (Endesfelder et al. 2011). Hence, supplementing the imaging buffer with thiols was evaluated for the influence on the number of blinking events of fluorescent proteins mEos3.2 and mMaple3. While the number of blinking events has increased for mEos3.2 $(p=0.17)$, it decreased for mMaple3 ( $p=0.55)$ (Figure 4.1.4) 

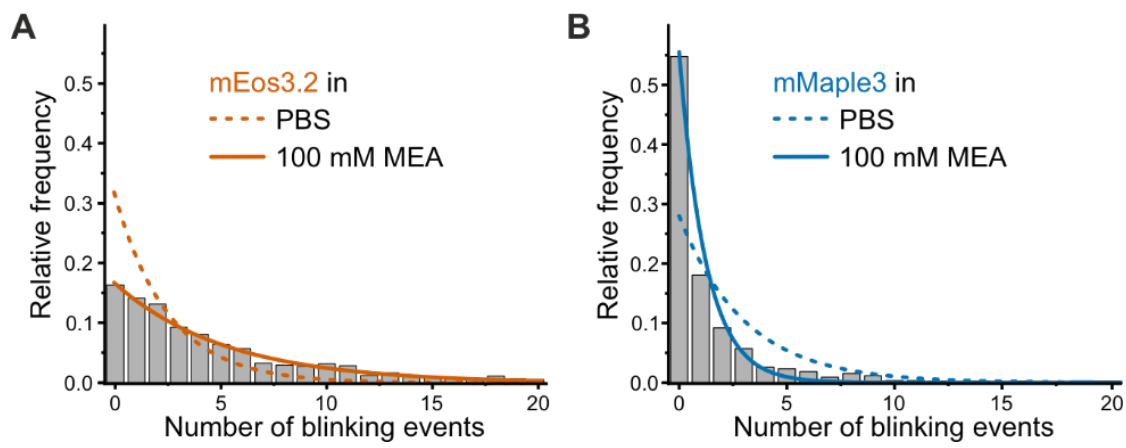

Figure 4.1.4: Effect of reducing thiols on the blinking properties of mEos3.2 and mMaple3. Histograms of the relative frequencies of the number of blinking events for mEos3.2 (A) and mMaple3 (B) fitted by equation 11 to obtain the $p$-values in PBS supplemented with $100 \mathrm{mM}$ MEA. Measurements in pure PBS buffer are shown in dashed lines as reference. Adapted from Baldering et al (2019b). Copyright CC BY-NC-SA 3.0.

It was shown that several amino acids in the vicinity of the chromophore of fluorescent proteins can influence the photophysical properties (Turkowyd et al. 2017). It is thus likely that blinking properties will also be affected. The amino acid 69 (in Dendra2, threonine) promotes primed conversion, an alternative process of photoconversion of fluorescent proteins without the need of violet light (Turkowyd et al. 2017). Thus, the blinking properties in response to modifications in this amino acid were of interest. The common amino acid for mEos3.2 is alanine and for Dendra2 is threonine. Mutants of mEos3.2 and Dendra2 were generated in the lab of Ulrike Endesfelder (MPI for Terrestrial Microbiology, Marburg) and the $p$-values were measured. The amino acid 69 turned out to affect the blinking properties of mEos3.2 and Dendra2. Dendra2T69A showed higher number of blinking events $(p=0.33)$ similar to wildtype mEos3.2, whereas the mutant mEos3.2A69T exhibited fewer blinking cycles $(p=0.55)$ (Figure 4.1.5). The $p$-values of Dendra2T69A and mEos3.2 as well as Dendra2 and mEos3.2A69T differed only slightly $(\Delta p<0.02)$.
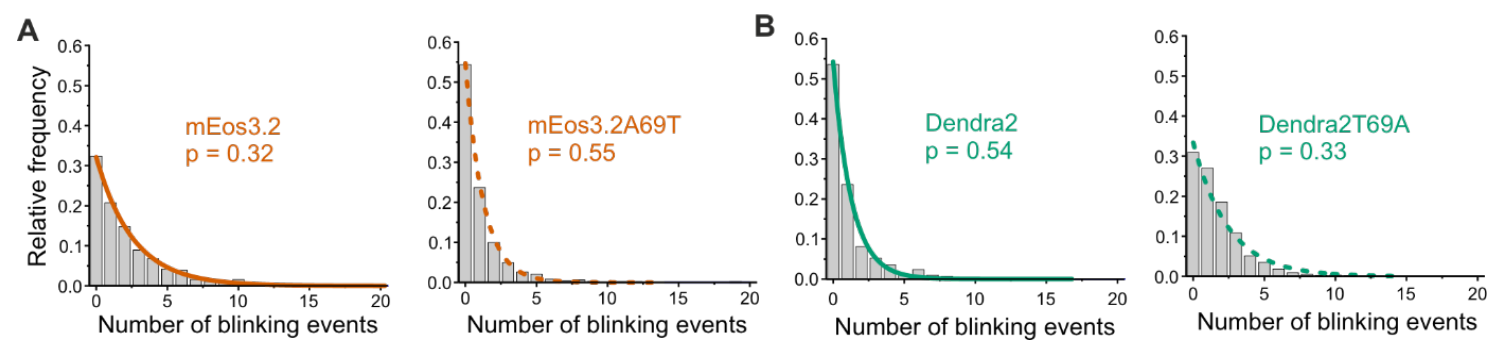

Figure 4.1.5: Blinking properties of mEos3.2A69T and Dendra2T69A. Histograms of the relative frequencies of the number of blinking events are shown for (A) mEos3.2 (left), 


\section{Results \& Discussion}

mEos3.2A69T (right) and (B) Dendra2 (left) and Dendra2T69A (right). The determined $p$-values are displayed in the respective histograms.

An additional parameter for qPALM is the mean photon yield of a fluorescent protein. Comparing the photon yield of mEos3.2, mMaple3, Dendra2, and PAmCherry2 (Figure 4.1.6), highest photon yield was observed for mEos3.2 (mean photon counts $\sim 1100$ ), while the photon yields of the other fluorescent proteins were halved (mean photon counts $\sim 600$ ).

A
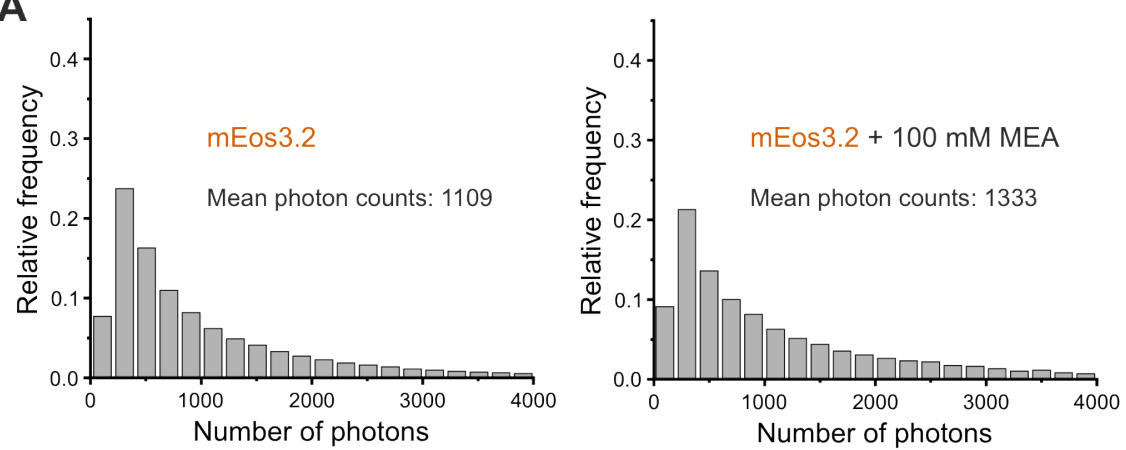

B
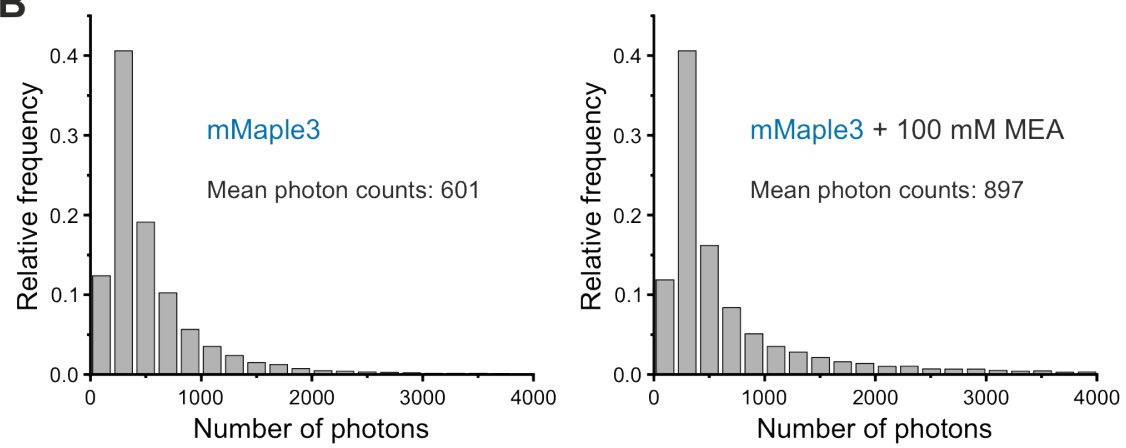

C
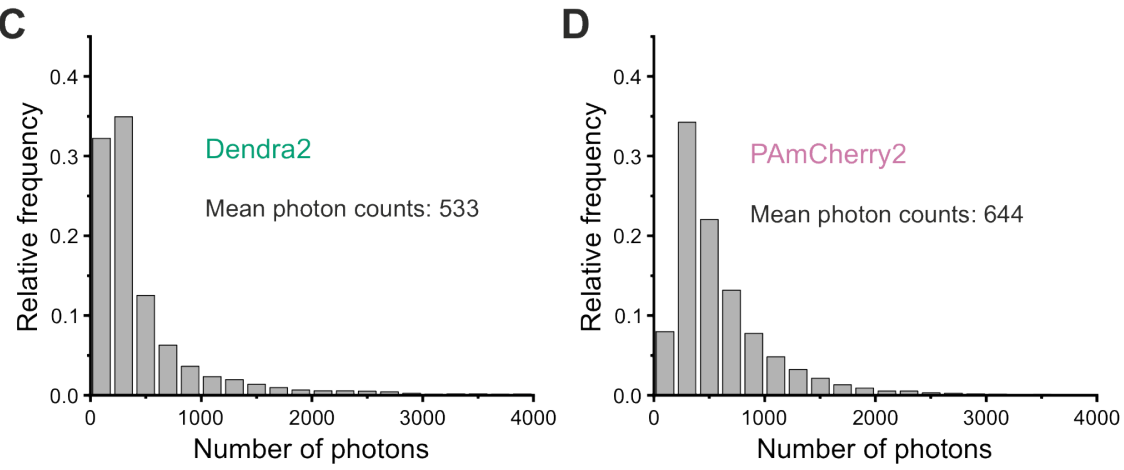

Figure 4.1.6: Photon counts of different fluorescent proteins and with different buffer conditions. Histogram of the photon count distribution of mEos3.2 (A), mMaple3 (B), Dendra2 (C), and PAmCherry2 (D). Adapted from Baldering et al (2019b). Copyright CC BY-NC-SA 3.0. 
In summary, these results show that different fluorescent proteins cover a range of $p$ values. In addition, $p$-values were consistent for different variants of the Eos family and if measured with different microscopes. At the same time, the blinking properties are influenced in the presence of reducing agents or specific mutations in the region of the chromophore.

\subsubsection{Synthetic and genetic dimers as quantification rulers for qPALM experiments}

The determination of protein numbers in assemblies requires calibrations with proteins at defined stoichiometries. In addition, proteins do not always fold correctly resulting in aggregates or inactive molecules, which makes quantification challenging. Fluorescent proteins might be misfolded or contain an unmatured chromophore and thus be nonfluorescent. This fraction of inactive fluorescent proteins leads to undercounting in e.g. dimers, the assembly of two proteins. Hence, a correction term is required that is determined from calibration proteins that form e.g. exclusively dimers. In addition to the bleaching probability, the $q$-value is of high importance for the proper analysis of qPALM data as it corrects for inactive fluorescent proteins and was first described in Hummer et al. (2016). To determine this value for $\operatorname{mEos} 3.2$, synthetic and genetic dimers were constructed (Figure 4.1.7 A). The synthetic dimer was generated by the linkage of two His-tagged mEos3.2 molecules to a DNA linker that contained tris-NTA moieties on both sides. The generation of this DNA linker was verified in an agarose gel (Figure App.4.1.1). The synthetic dimer contained two mEos3.2 molecules and was purified by size-exclusion chromatography (Figure App.4.1.1). The generation, gel analysis and purification of the synthetic dimer was performed by Karl Gatterdam (AK Tampé). qPALM imaging on a single-molecule surface revealed a higher number of blinking events compared to monomeric mEos3.2 (Figure 4.1.7 B and Figure 4.1.2 A). The histogram was fitted by equation 12 with a fixed $p$-value of $p=0.32$, yielding a $q$-value of $0.36 \pm 0.03$. In order to confirm the determined $q$-value with a second calibration reference, a genetic dimer was cloned consisting of two mEos3.2 molecules connected by a short peptide linker (Figure 4.1.7 A, bottom). The genetic dimer was expressed in E.coli, purified by a Ni-NTA column and applied onto an SDS-PAGE gel. The purified protein contained about $20 \%$ of monomers and $80 \%$ of dimer, according to the SDSPAGE gel (Figure 4.1.7 C). This ratio was then compared to the results of the qPALM measurement. The histogram of the number of blinking events of the genetic dimer was fitted with $p=0.32$ and $q=0.36$ using a fit model accounting for a mixture of 
monomers and dimers. $82 \pm 5 \%$ dimers and $18 \pm 5 \%$ monomers were determined from the qPALM data (Figure 4.1.7 C). The monomer and dimer fractions estimated from the SDS gel and the qPALM experiments are very similar and confirm the plausibility of the $q$-value determined from the synthetic dimer. In addition, it shows that estimations of monomer/dimer ratios with qPALM are possible.
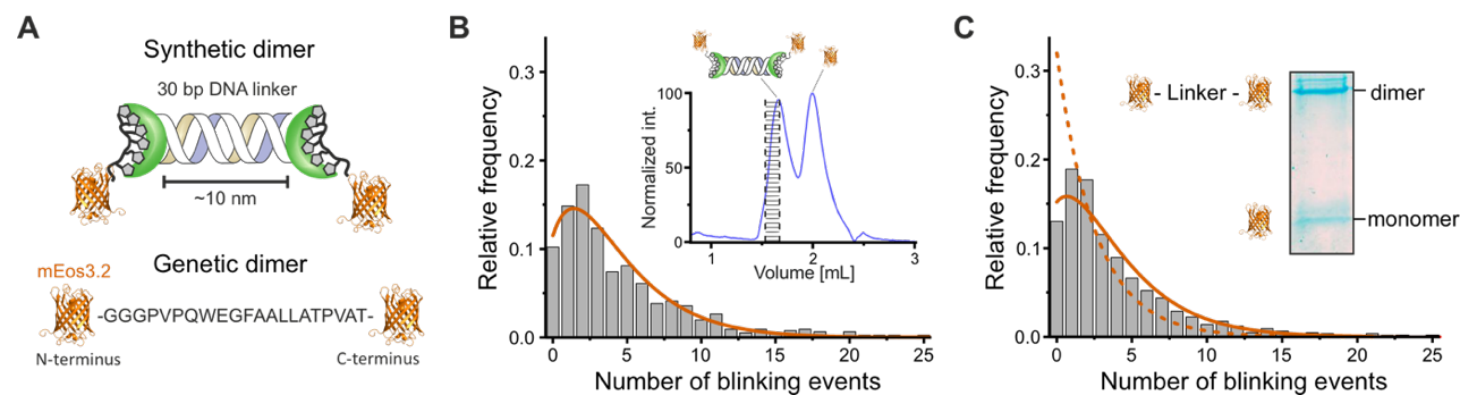

Figure 4.1.7: Synthetic and genetic dimers as quantification rulers for the determination of the $q$-value in qPALM experiments. (A) Schematic illustration of the synthetic and genetic dimer. The synthetic dimer connects two His-tagged mEos3.2 molecules (orange, crystal structure of mEos2, PDB: 3S05) via a 30-base pair DNA linker consisting of two tris-NTA moieties (green) on both sites. The genetic dimer was designed as two mEos3.2 proteins with a short peptide linker in between. (B) Histogram of the relative frequency of the number of blinking events of the synthetic dimer was approximated by equation 12 . Inset: The size-exclusion chromatogram shows the purified fraction (marked in black). (C) Histogram of the relative frequency of the number of blinking events of the genetic dimer approximated by a linear combination of monomer and dimer functions with $p=0.32$ and $q=0.36$ (orange solid line). The approximation for monomeric mEos3.2 is depicted as orange dotted line. An SDS gel of the purified genetic dimer is shown containing dimeric and monomeric mEos3.2. Adapted from Baldering et al (2019b). Copyright CC BY-NC-SA 3.0.

\subsubsection{In cellulo calibration rulers for the determination of qPALM parameters}

Next, evaluation of the variability of the qPALM parameters $p$ and $q$ were addressed in cellulo. For that purpose, fusion proteins of cellular reference proteins with mEos3.2 and mEos4b were cloned and transfected into HeLa and HEK293T cells (Figure 4.1.8 and 4.1.8). The protein CD86 was shown to be mainly monomeric (Bhatia et al. 2005; James et al. 2006; Dorsch et al. 2009; Girard et al. 2014) and was thus used for the determination of the $p$-value. CTLA-4 was reported to organize into dimers (Linsley et al. 1995; Greene et al. 1996; James et al. 2006), and was used to determine the $q$-value. qPALM videos were recorded $(\mathrm{N}=10)$ and clusters were analyzed for the number of blinking events. For 
CD86-mEos3.2, the histogram was approximated by equation 11 and a $p$-value of $0.27 \pm$ 0.01 was obtained (Figure 4.1.8 A), which deviates from the $p$-value determined in vitro $(p=0.32)$ by $16 \%$. This $p$-value was used to approximate the blinking number distribution of CTLA-4-mEos3.2 by equation 12. A $q$-value of $0.39 \pm 0.01$ was determined (Figure 4.1.8 B), which is similar to the $q$-value determined from the synthetic dimer. The blinking histograms of CTLA-4-mEos3.2 were measured and analyzed by Christos Karathanasis. The same procedure was performed with CD86-mEos4b and CTLA-4-mEos4b (Figure 4.1.9). With CD86-mEos4b the same $p$-value was determined as for CD86-mEos3.2 ( $p=0.27)$. The approximation of the CTLA-4-mEos4b histogram yielded a $q$-value of 0.35 , which differs slightly from the $q$-value of CTLA-4-mEos3.2. The qPALM measurements of CD86-mEos4b and CTLA-4-mEos4b were partially performed and fully analyzed by Christos Karathanasis.

In summary, for mEos3.2, the bleaching probability $p$ differs by $16 \%$, and the $q$-value differs by $8 \%$ between in vitro and in cellulo experiments. Moreover, the two parameters for $m E \operatorname{los} 4 \mathrm{~b}$ are similar to the parameters obtained for mEos3.2.
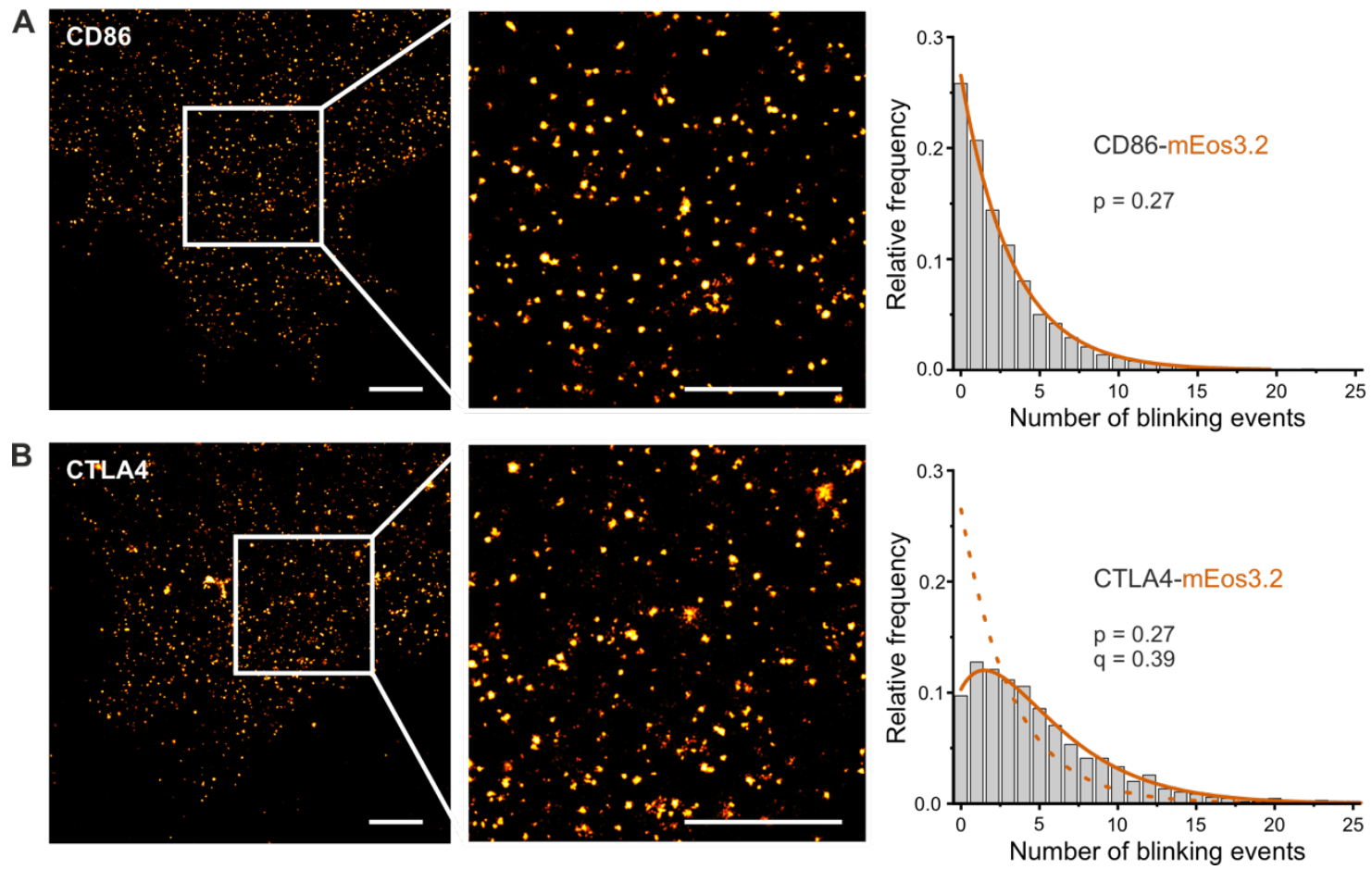

Figure 4.1.8: CD86-mEos3.2 and CTLA-4-mEos3.2 serve as in cellulo calibration rulers for qPALM. PALM images of CD86-mEos3.2 (A) and CTLA-4-mEos3.2 (B) transfected into HeLa cells (left site). Zoom-ins of representative areas are shown in the middle panel. Histograms of the relative frequency of the number of blinking events are shown on the right side. CD86mEos3.2 data was approximated by the monomeric function (equation 11) and CTLA-4-mEos3.2 


\section{Results \& Discussion}

was approximated by the dimer function with $p=0.27$ (equation 12). The $q$-value was determined with the approximation of equation 12 to the blinking histogram. Adapted from Baldering et al (2019b). Copyright CC BY-NC-SA 3.0.
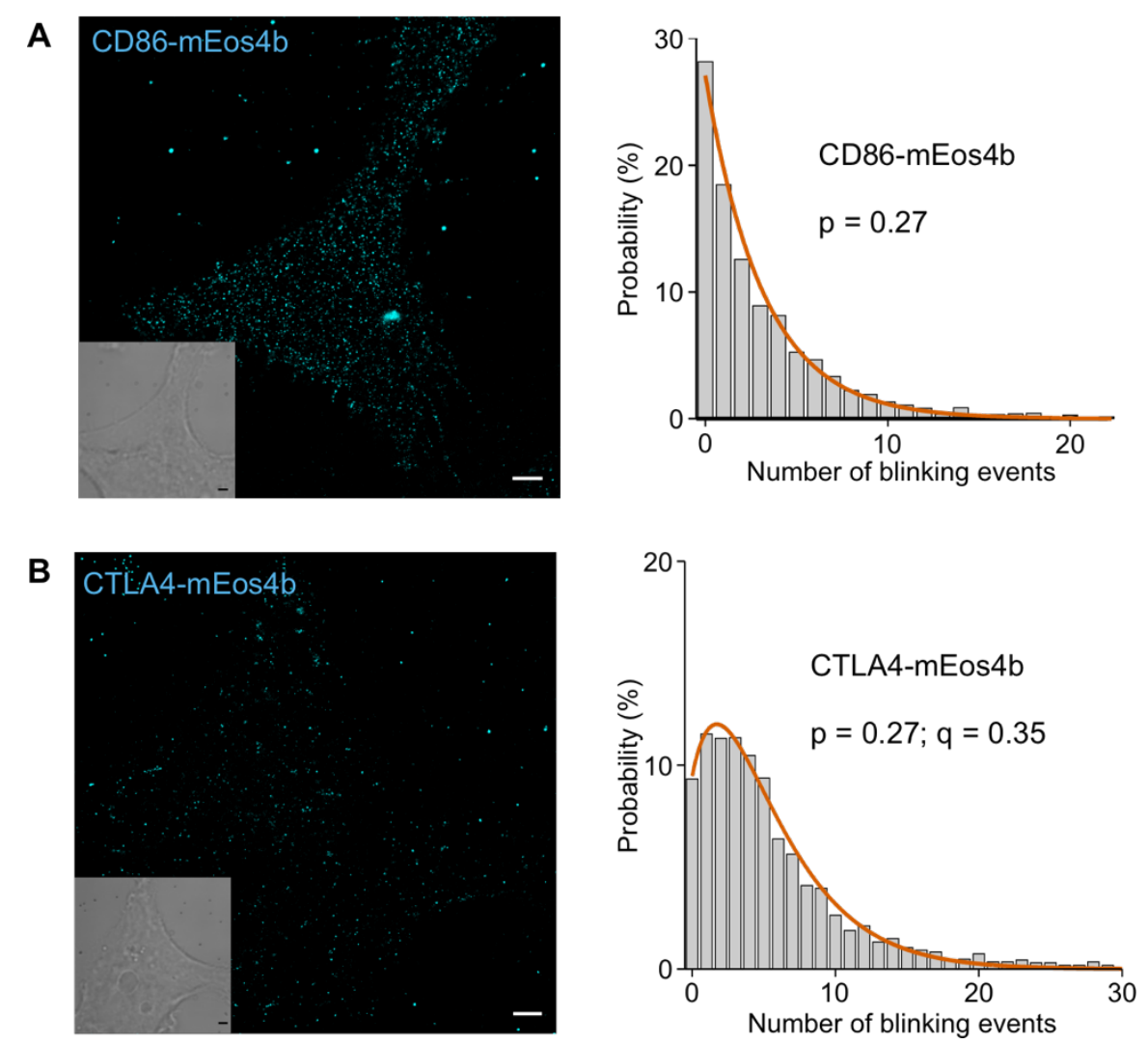

Figure 4.1.9: CD86-mEos4b and CTLA-4-mEos4b serve as in cellulo calibration rulers for qPALM. PALM images of CD86-mEos4b (A) and CTLA-4-mEos4b (B) transfected into HEK293T cells (left site). Histograms of the relative frequency of the number of blinking events are shown on the right side. CD86-mEos $4 \mathrm{~b}$ data was approximated by the monomeric function (equation 11) and CTLA-4-mEos4b was approximated by the dimer function with $p=0.27$ (equation 12). The $q$-value was determined with the approximation of equation 12 to the blinking histogram. Adapted from Baldering et al (2020). Copyright CC BY 4.0.

\subsubsection{Simulations of higher-ordered oligomers and mixtures of monomers and dimers}

In order to investigate the performance of qPALM on higher-order oligomers, clusters of the monomeric mEos3.2 blinking number were randomly grouped to generate simulated dimers, trimers, and tetramers. The sum of the number of blinking events of the combined clusters was used as synthetic spots. This produced histograms of synthetic oligomers that were approximated with linear combinations of equation 10 with $p=0.32, q=0$, and the oligomer complexity $m(m=0 ; 1$ for dimers, $m=0 ; 1 ; 2$ for trimers and $m=$ 
$0 ; 1 ; 2 ; 3$ for tetramers. The weights of the individual terms for each oligomer reflect the fraction of monomers, dimers, trimers, or tetramers. In the case of synthetic generated dimers, about 98\% dimers were found (Figure 4.1.10 A, left). In addition, mixtures of monomers and dimers were analyzed in which the analysis accords well with the ground truth (Figure 4.1.10 B). However, in synthetic trimers and tetramers, the trimer and tetramer fraction deviated by about $10-15 \%$ from the ground truth $(100 \%$ trimers/tetramers) (Figure 4.1.10 A, middle and right). This divergence has been examined in more detail in section 4.2.
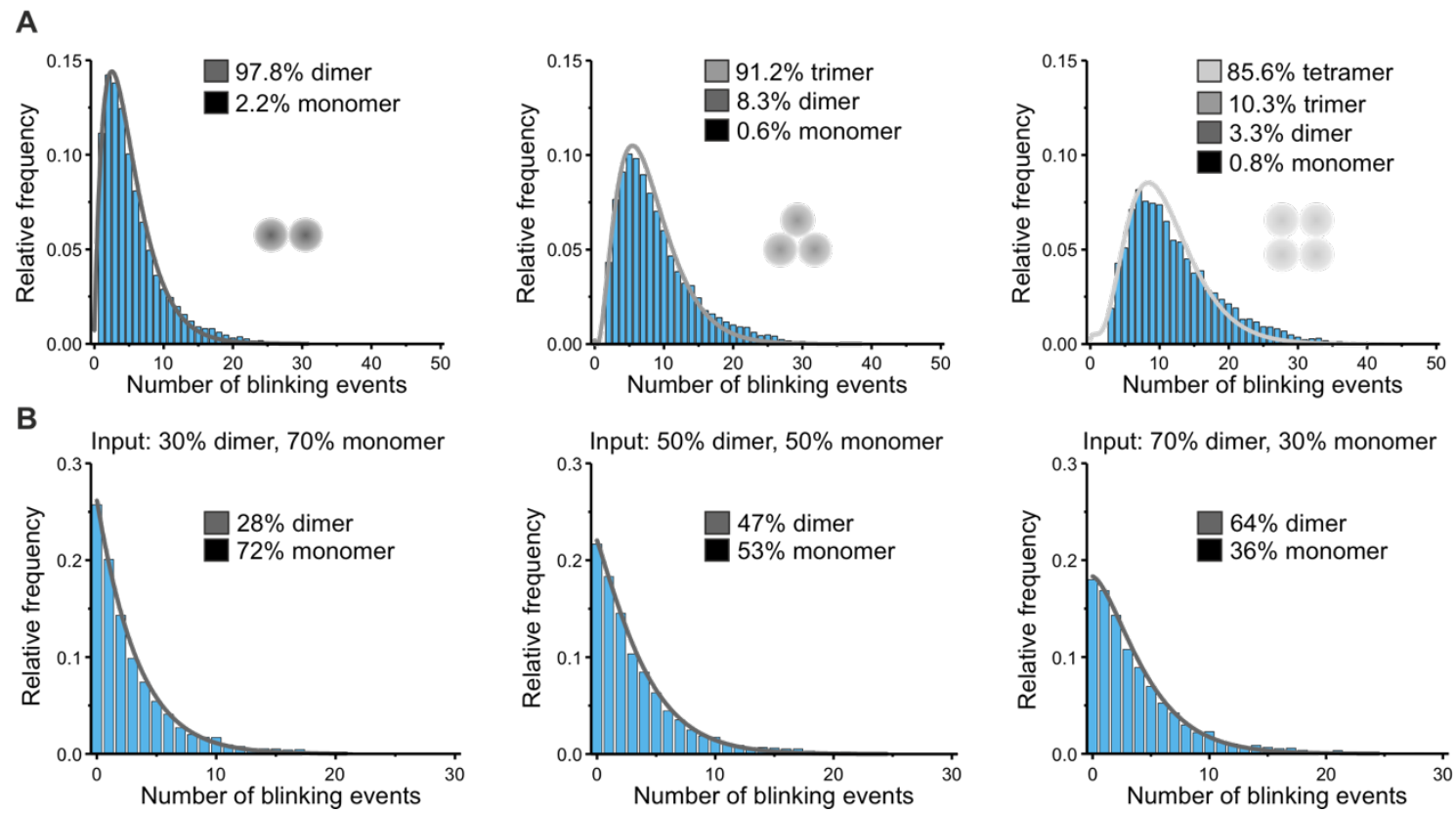

Figure 4.1.10: Simulations of higher-ordered oligomers and mixtures of monomers and

dimers. (A) Generation of dimers (left), trimers (middle) and tetramers (right) from monomeric mEos3.2 blinking numbers. The histograms were approximated with a linear combination of monomer and dimer function, monomer, dimer, and trimer function, or monomer, dimer, trimer, and tetramer function, respectively. (B) Synthetic combinations of monomers and dimers in different percentages as ground truth (input values). The histograms were approximated with a linear combination of monomer and dimer fit functions revealing the monomer/dimer ratios as output. Adapted from Baldering et al (2019b). Copyright CC BY-NC-SA 3.0.

\subsubsection{Discussion}

Quantitative analysis of protein stoichiometries by single-molecule localization microscopy provide important information about biological systems but are challenging due to aspects such as over- and undercounting. qPALM corrects for both over- and undercounting by implementing two main parameters (Fricke et al. 2015; Hummer et al. 
2016): the bleaching probability ( $p$-value) describes the photokinetics of a fluorescent protein, and the $q$-value considers undetected molecules. Here, the two critical parameters, $p$ and $q$, were characterized in vitro and in cellulo.

\section{Blinking properties of different fluorescent proteins}

$p$-values of different fluorescent proteins were measured. mEos3.2 $(p=0.32)$ and mMaple3 ( $p=0.28$ ) showed a higher number of blinking events compared to Dendra2 $(p=0.54)$ and PAmCherry2 $(p=0.77)$ (Figure 4.1.2). The $p$-values for mMaple3 and PAmCherry2 are comparable with previous studies that showed mean number of blinking events of 3.4 for mMaple and 1.0 for PAmCherry (Durisic et al. 2014). The mean number of blinking events for Dendra2 (2.7) compared to mEos3.2 (2.4) reported in Durisic et al. (2014) differ from the results presented here. Possible explanations could be experimental parameters such as different excitation wavelength and emission filter, which lead to different photon yields of the individual fluorophores. Hence, lower photon yields may not exceed the photon thresholds and are not considered in the analysis.

It is tempting to assume that PAmCherry is optimal for qPALM experiments, since almost no overcounting is observed, because the majority of PAmCherry showed a single emission event. In addition, efforts were made to minimize overcounting of mEos 2 by introducing a dark time $\left(t_{d}\right)$ (Annibale et al. 2011). However, a dark time may lead to dimeric fluorescent proteins analyzed as a single protein. Furthermore, the differentiation between signal of fluorophores and background signal is more difficult at lower emission events. Therefore, small $p$-values are beneficial for qPALM because a better distinction between fluorophore signal and background signal is possible. Possible candidates for qPALM therefore comprise mEos3.2 and mMaple3.

Next, different variants of the Eos family were analyzed. The $p$-value of mEos $4 \mathrm{~b}$ ( $p=$ 0.34 ) is similar to the $p$-value of mEos3.2 (Figure 4.1.2 A, 4.1.3 A) and a previously reported $p$-value for $\operatorname{mEos} 2(p=0.29)$ (Fricke et al. 2015; Hummer et al. 2016). This is to be expected, since these variants only differ in a few amino acids. For example, mEos3.2 was derived from mEos2 with mutations in the amino acids I102N, H158E, and Y189A (Zhang et al. 2012). These amino acids do not belong to the chromophore or point outwards from the $\beta$-barrel structure and should therefore not influence the blinking kinetics. The variant mEos4b comprises more mutations (M1MV, K9R, F34Y, S39T, $\mathrm{A} 69 \mathrm{~V}, \mathrm{C} 195 \mathrm{~A})$, in which the mutation of amino acid 69 could influence the photophysics of the chromophore (Paez-Segala et al. 2015). However, alanine and valine are similar 
amino acids which both represent hydrophobic side chains. Therefore, no difference in blinking properties is expected for mEos $4 \mathrm{~b}$ in addition to mEos 3.2 and mEos 2 . These considerations are supported by single-molecule data that demonstrates similar $p$-values for $\mathrm{mEos} 2, \mathrm{mEos} 3.2$, and $\mathrm{mEos} 4 \mathrm{~b}$.

Reducing thiols and mutations in residue 69 influence the blinking properties of mEos3.2, mMaple3 and Dendra2

The combination of PALM with dSTORM can enable multicolor experiments. However, dSTORM requires reducing agents such as MEA at concentrations of 10-100 mM to modulate photoswitching of organic fluorophores. Therefore, it is interesting to analyze the influence of such buffers on the blinking properties of mEos3.2 and mMaple3. In the presence of $100 \mathrm{mM}$ MEA, a lower $p$-value was determined for mEos3.2 ( $p=0.17)$, which is consistent with more blinking events in the presence of MEA observed in a previous study for mEos2 (Endesfelder et al. 2011). In contrast, a higher $p$-value was determined for mMaple3 ( $p=0.55$ ), which corresponds to fewer blinking cycles. This difference can be explained by different amino acid sequences that influence the constitution of the chromophore (Subach und Verkhusha 2012), thus leading to e.g. stabilized forms of the dark and fluorescent states. In particular, the residue 69 could be of importance, which differs in mEos3.2 (alanine) and mMaple3 (threonine) and which is assumed to have a catalytic function in the formation of the red chromophore (Stepanenko et al. 2013). Furthermore, this residue is in close proximity to the amino acids His62-Tyr63-Gly64, which constitute the chromophore, and residue 69 could possibly influence the stability of the chromophore (Pletneva et al. 2016; Bourgeois 2017).

Residue 69 is also responsible for primed conversion with excitation wavelengths of 488/720 nm (Turkowyd et al. 2017). Mutants of mEos3.2 and Dendra2, mEos3.2A69T and Dendra2T69A, were analyzed to investigate the influence of residue 69 especially in combination with their ability to perform primed conversion. The $p$-values interchanged between mutants and wildtype proteins (Figure 4.1.5), which implies that amino acid 69 may have a major influence on the blinking properties of fluorescent proteins. The amino acid alanine lead to higher blinking events $(p=\sim 0.32)$ but is unable to perform primed conversion. In comparison, threonine resulted in fewer blinking cycles $(p=\sim 0.54)$ and allowed primed conversion (Turkowyd et al. 2017). However, further experiments, e.g. 
on kinetic crystallography, could be useful to decipher the complete mechanism of blinking, the associated dark states, and rate constants (Bourgeois 2017).

\section{Applications of qPALM for quantitative imaging in cells}

In addition to high numbers of blinking events, a high photon budget is an advantage for SMLM, because the localization precision is improved. The photon yield of the different fluorescent proteins was investigated, showing that mEos3.2 emits substantially more photons than mMaple3, Dendra2 and PAmCherry2. The large difference can be in parts explained by the emission filter set $(590 \pm 10 \mathrm{~nm})$, which is optimal for the emission spectra of the Eos family (peak at $\sim 580 \mathrm{~nm}$ ). Consistently, other studies also showed that mEos3.2 has a higher photon yield compared to the other fluorophores, such as mMaple (Wang et al. 2014).

The fluorescent protein mEos3.2 showed the highest photon number and thus was used to generate different types of dimers to determine the $q$-value of this fluorescent protein in vitro. The $q$-value, which estimates the fraction of undetected molecules, was determined to 0.36 with the synthetic mEos3.2 dimer (Figure 4.1.7). This $q$-value reflects a detection efficiency that is slightly higher compared to detection efficiencies published in other studies for the Eos family (40-60\%, (Puchner et al. 2013; Durisic et al. 2014)). However, a higher detection efficiency of $\sim 80 \%$ was published for eGFP (Ulbrich und Isacoff 2007). Hence, the detection efficiency is in the range of published values. Nevertheless, the detection efficiency could be influenced by various parameters such as the linker length between fusion proteins or the nano-environment and thus vary between different fusion proteins.

Fusion proteins of reference proteins with known stoichiometry were generated to determine the $p$ - and $q$-values of mEos3.2 in cellulo (Figure 4.1.8). The analysis of CD86mEos3.2 revealed a $p$-value of 0.27 , which is similar to the $p$-value $(0.28)$ observed for CD86-mEos2 in HeLa cells (Fricke et al. 2015; Hummer et al. 2016). The $p$-values were measured for mEos3.2 and mEos $4 \mathrm{~b}$ in vitro and were slightly higher compared to the $p$ values in cellulo. This difference can be explained by a different environment of the fluorescent protein inside a cell compared to a homogenous buffer in single-molecule experiments on glass. The $p$-value obtained for CD86-mEos3.2 enabled the determination of the $q$-value of 0.39 from dimeric CTLA-4-mEos3.2. A lower $q$-value of 0.30 was observed for CTLA-4-mEos2 (Fricke et al. 2015; Hummer et al. 2016). This difference can be explained by different maturation efficiencies of mEos 2 and mEos3.2. A smaller 
difference was found between the $q$-value of $\mathrm{mEos} 3.2$ in vitro $(q=0.36)$ and in cellulo $(q=0.39)$. The higher $q$-value in cells can be explained by a higher amount of inaccurate folding of the fusion protein, since the fluorescent protein alone should fold faster and more efficiently. A lower detection efficiency was also observed for mEos3.2 in photobleaching experiments (photoactivation efficiency of 41\%) (Durisic et al. 2014), which provides additional evidence for inefficient folding of fusion proteins. Similar $p$ and $q$-values of CD86-mEos2 and CTLA-4-mEos2 were also determined in HEK293 and MEF cells (Krüger et al. 2017; Karathanasis et al. 2020) and thus demonstrate the robustness of this approach. Additionally, the $p$ - and $q$-values of CD86-mEos $4 \mathrm{~b}$ and CTLA-4-mEos4b $(p=0.27, q=0.35)$ are very similar to that of CD86-mEos3.2 and CTLA-4-mEos3.2. However, for precise determinations of oligomeric fractions, the $p$ and $q$-values should be analyzed in the respective cell line.

In summary, different fluorescent proteins and variants of the Eos family were analyzed regarding their blinking kinetics. Blinking kinetics were investigated with two different microscopes and in two buffer conditions. Synthetic and genetic dimers were generated in vitro and enabled the determination of the $q$-value for mEos3.2. The two parameters, $p$ and $q$, were further investigated in cellulo and small deviations were found in comparison to the in vitro measurements. As an overview, all $p$ - and $q$-values are listed in table 4.1.1. Simulations of higher-ordered oligomers were generated from monomeric mEos3.2 data, exploring the applicability to determine monomer/dimer mixtures and higher-ordered oligomers.

Table 4.1.1: $p$ - and $q$-values of various fluorescent proteins in vitro and in cellulo.

\begin{tabular}{|l|c|c|c|}
\hline \multicolumn{1}{|c|}{ Condition } & $\boldsymbol{p}$-value & $\boldsymbol{q}$-value & $\begin{array}{c}\text { Fraction of } \\
\text { monomer/dimer }\end{array}$ \\
\hline mEos3.2 & 0.32 & - & - \\
\hline mEos4b & 0.34 & - & - \\
\hline mMaple3 & 0.28 & - & - \\
\hline Dendra2 dimer & 0.54 & - & - \\
\hline PAmCherry & 0.77 & 0.36 & \\
\hline $\begin{array}{l}\text { Synthetic } \\
\text { mEos3.2 }\end{array}$ & 0.32 & & - \\
\hline
\end{tabular}


4 Results \& Discussion

\begin{tabular}{|l|c|c|c|}
\hline $\begin{array}{l}\text { Genetic dimer } \\
\text { mEos3.2 }\end{array}$ & 0.32 & 0.36 & $\begin{array}{c}18 \% \text { monomer, } 82 \% \\
\text { dimer }\end{array}$ \\
\hline CD86-mEos3.2 & 0.27 & - & - \\
\hline CD86-mEos4b & 0.27 & - & - \\
\hline CTLA-4-mEos3.2 & 0.27 & 0.39 & - \\
\hline CTLA-4-mEos4b & 0.27 & 0.35 & - \\
\hline
\end{tabular}




\subsection{Extending the theory of qPALM}

The detection efficiency is an important parameter when analyzing the oligomeric state of proteins. A fraction of fluorophores can be inactive due to a defective fluorophore and lead to counting errors. Especially when analyzing higher-ordered oligomers, accurate knowledge of the detection efficiency is required. The initial model of qPALM (Hummer et al. 2016) introduced $q$ as the fraction of inactive fluorophores. The analysis of simulations for higher-ordered oligomers (Figure 4.1.10 A) indicated that this model leads to inaccuracies with higher-ordered oligomers. The theory of qPALM was studied and inaccuracies with the application of the q-value at higher-ordered oligomers were found. In this section, a revised model is presented that directly reports a detection probability (Model 2, Figure 4.2.1). Furthermore, the relation of the $q$-value and the detection efficiency is investigated. Undetected oligomers lead to small variations in the analysis of mixtures of different oligomers. These variations are quantified here, and it is shown that the detection efficiency is useful to correct for undetected oligomers. The revised model enables the exact determination of mixtures of complex oligomers in simulated qPALM data generated from monomeric mEos3.2 data. Finally, the influence of the assignment of the $q$-value and the influence of undetected oligomers on previous studies is discussed.

A Model 1

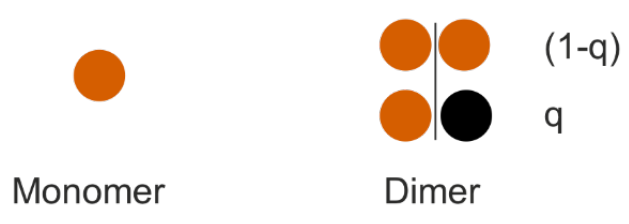

B

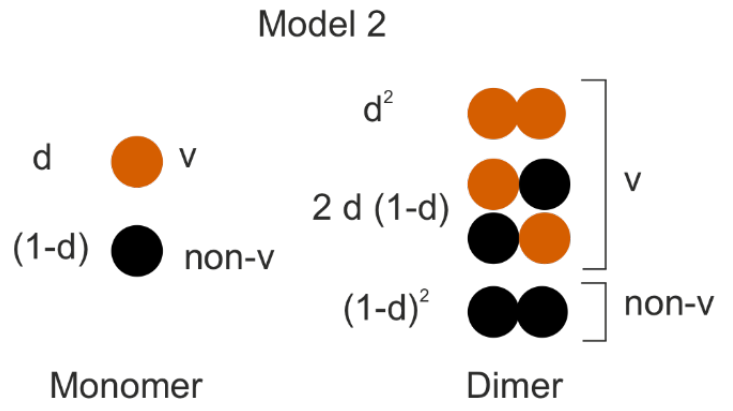

Figure 4.2.1: Model comparison for qSMLM analysis. The two models are compared on the example of monomers and dimers. Fluorescent molecules are shown in orange and undetected molecules as black circles. (A) Schematic illustration of the $q$-value in model 1, which describes the fraction of dimers with one inactive fluorophore (Hummer et al. 2016). (B) Schematic illustration of the detection efficiency $d$ implemented in model 2. The probabilities for the detection of dimers with different numbers of active and inactive chromophores are given. Here, $v$ is the fraction of visible oligomers while non- $v$ represents the fraction of invisible oligomers. Adapted from Baldering et al (2019a). Copyright CC BY 4.0. 


\subsubsection{A comparison of two models that describe simulated qPALM data}

To evaluate possible variations of the two models, Monte-Carlo simulations were performed with a home-written software (stochastic simulation of photophysics tool, ssp, created by Sebastian Malkusch) (for details see section 3.22). Simulations were performed by assuming the 4-state stochastic model for fluorescent proteins (Figure 4.1.1 A) with a defined bleaching probability $(p=0.3)$ and a probability for detecting a molecule of $d=0.57$. These values were chosen based on previously experimentally determined parameters (see section 4.1 for details; Figure 4.1.3). For each simulation, a defined number of oligomers $(\mathrm{N})$, the order of each oligomer type $(m+1)$ and its fraction $\left(y_{m}\right)$ was specified. First, an ideal trimer was simulated and the relative frequency of number of blinking events were depicted in a histogram. The overlay of model 1 and model 2 (with fixed parameters of $p=0.3 ; q=0.43$ for model 1 and $d=$ 0.57 for model 2) showed that the histogram of the simulated trimer was well described by model 2, whereas model 1 showed deviations (Figure 4.2.2 A). The different ability to describe the simulated qPALM data are highlighted by the residuals shown in Figure 4.2.2 B. In contrast, model 2 showed almost no deviations (Figure 4.2.2 B) and the residuals were normally distributed around zero (Figure 4.2.2 C).
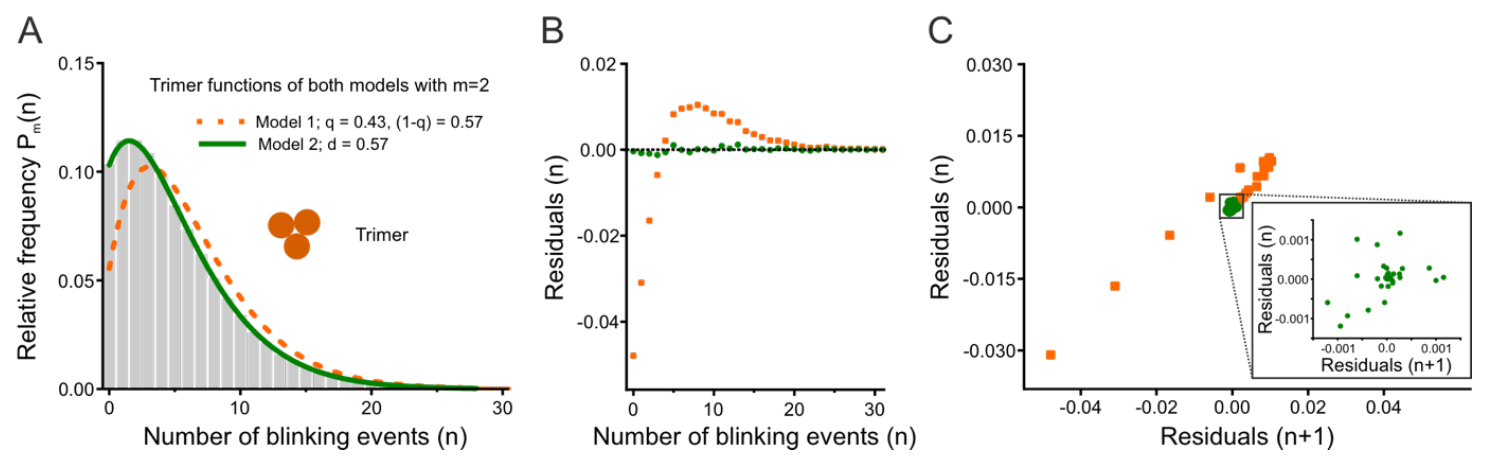

Figure 4.2.2: A comparison of model 1 in describing the blinking data of a simulated trimer.

(A) Histogram of the relative frequency of the number of blinking events for a simulated trimer. Model 1 is shown as orange dashed line and model 2 is shown as green solid line. Input parameters for both models are depicted in the graph. (B) Residuals of model 1 and 2 showing the deviations to the simulated data. (C) Lag plot of the residuals of model 1 and 2. Residuals of the number of blinking events $n$ are plotted against the residuals of $n+1$ for both models to evaluate systematic deviations of the model from the data. Adapted from Baldering et al (2019a). Copyright CC BY 4.0. 
Systematic deviations of model 1 were also observed for higher-ordered oligomers, e.g. tetramers, hexamers and nonamers (Figure 4.2.3). Consequently, the $q$-value does not sufficiently reflect the fraction of undetected molecules.

A
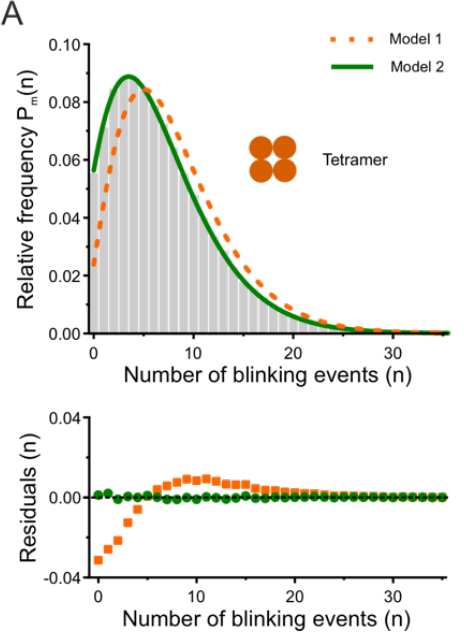

B
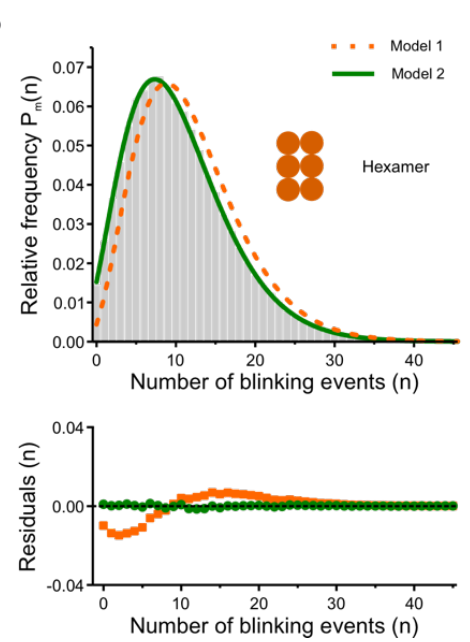

C
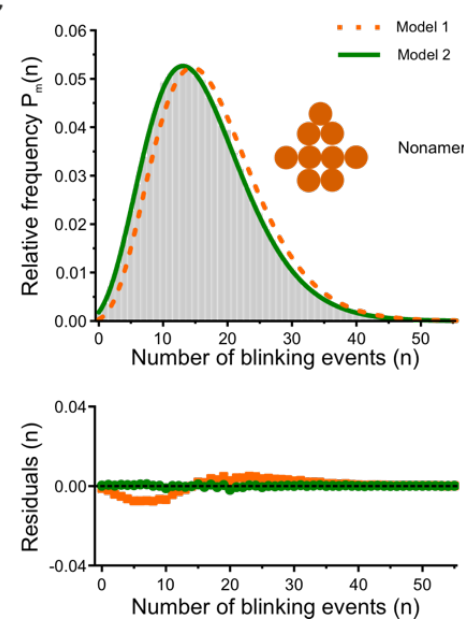

Figure 4.2.3: A comparison of model 1 in describing the blinking data of higher-ordered

oligomers. Histogram of the relative frequency of the number of blinking events for simulated qPALM data of a tetramer (A), hexamer (B), and nonamer (C). Model 1 is shown as orange dashed line and model 2 is shown as green solid line. Parameters $p=0.3, q=0.43$, and $d=$ 0.57 were used for model 1 and 2, respectively. Adapted from Baldering et al (2019a). Copyright CC BY 4.0.

\subsubsection{Relation between the $q$-value (model 1) and the detection efficiency (model 2)}

In a dimer, the $q$-value and the detection efficiency $d$ can be substituted by each other. In model 1 (Figure 4.2.1 A), the $q$-value directly reflects the fraction of dimers consisting of only one active fluorophore. Hence, the $q$-value and the detection probability are related as (equation 18, 19; figure 4.2.1):

$$
\begin{aligned}
& q=\frac{2 \cdot d \cdot(1-d)}{2 \cdot d \cdot(1-d)+d^{2}} \\
& d_{\mid m=1}=\frac{2-2 q}{2-q}
\end{aligned}
$$

qPALM data of trimers and higher-ordered oligomers were simulated with $p=0.3$ and $d=0.57$, and approximated by model 1 and 2 to determine the $q$ - and $d$-values (Table 4.2.1). The obtained $q$-values were transformed into detection efficiencies by equation 19 
to determine the variations of the calculated detection efficiency to the ground truth of $d=0.57$.

Table 4.2.1: Detection efficiencies obtained by model 1 and 2 for oligomers of different order. qPALM data were simulated and the histograms of the relative frequency of the number of blinking events were approximated by model 1 and 2 to obtain the $q$ - and $d$-values. The $q$ values were transformed into detection efficiencies by equation 19. The ground truth detection efficiency is $d=0.57$.

\begin{tabular}{lccc}
\hline Oligomer & Model $1(\mathbf{q})$ & Model 1 (d) & Model 2 (d) \\
\hline Dimer & $0.599 \pm 0.030$ & $0.572 \pm 0.030$ & $0.572 \pm 0.030$ \\
Trimer & $0.582 \pm 0.025$ & $0.590 \pm 0.025$ & $0.568 \pm 0.024$ \\
Tetramer & $0.553 \pm 0.019$ & $0.617 \pm 0.018$ & $0.572 \pm 0.016$ \\
Hexamer & $0.508 \pm 0.014$ & $0.659 \pm 0.013$ & $0.576 \pm 0.012$ \\
Nonamer & $0.482 \pm 0.012$ & $0.682 \pm 0.010$ & $0.572 \pm 0.010$ \\
\hline
\end{tabular}

Model 1 and 2 described the qPALM data with small residuals (Figure 4.2.4 A; figure App.4.2.2). However, when calculating the detection efficiencies from the $q$-values, deviations were observed that increase with the order of the oligomeric state (Figure 4.2.4 $\mathrm{B}$, table 4.2.1). This shows that the relation of $q$ and $d$ (equation 19) only holds for dimers. Thus, the $q$-value cannot be assigned as a characteristic value of a fluorescent protein and must be determined separately for different oligomers; $q$-values determined from dimers cannot be used for higher oligomers. In contrast, the detection efficiency of model 2 is a characteristic value of a fluorescent protein and hence once determined can also be used for higher-ordered oligomers. 
A

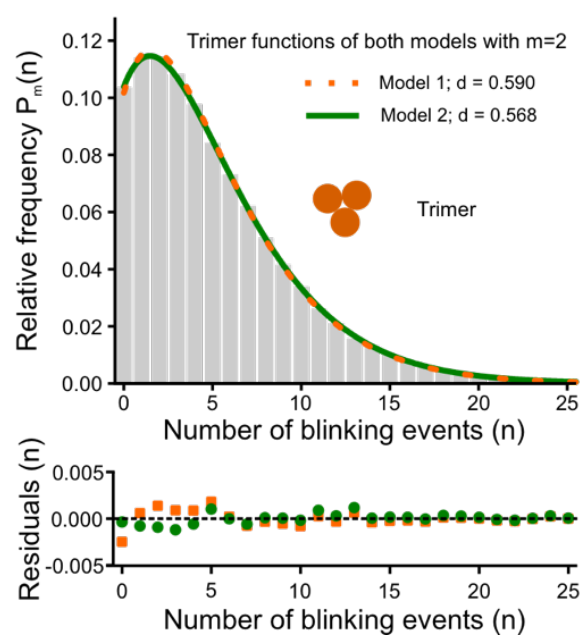

B

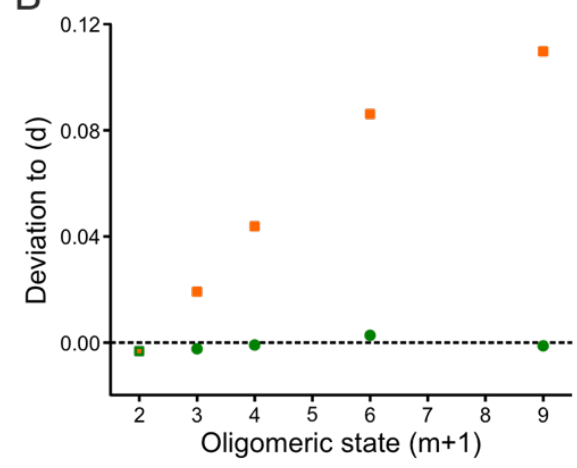

Figure 4.2.4: Comparison of determined detection efficiencies from model 1 and 2 for different oligomeric states. (A) Simulated qPALM data of trimers approximated by model 1 (orange dashed line) and 2 (green solid line) with $p=0.3$ and $q$ and $d$ were determined from the approximation. For comparison, the $q$-value was transformed into a detection efficiency by equation 19. (top) The residuals of model 1 and 2 are shown in their respective color (bottom). (B) Deviation of the detection efficiency from the ground truth $(d=0.57)$ for different orders of oligomeric states. Deviations of model 1 are shown in orange, deviations of model 2 are depicted as green squares. Adapted from Baldering et al (2019a). Copyright CC BY 4.0.

\subsubsection{Consideration of undetected oligomers in qPALM experiments}

Oligomers consisting exclusively of fluorescent proteins that are inactive and therefore not detected are not considered by the blinking analysis. In mixtures of different orders of oligomers, the undetected fractions differ and lead to deviations. The fraction of monomeric fluorophores that are not considered in the blinking analysis is described by $(1-d)$, whereas the fraction of higher-ordered oligomers is represented by $(1-d)^{m+1}$ as all fluorophores in the oligomer must be inactive for the whole oligomer to be not detected. The detection efficiency allows to correct for this and to obtain the true fractions.

The deviations in the determined oligomeric fractions was quantified in a mixture of monomers and dimers, each represented at the same proportions. The histogram of the relative frequency of the number of blinking events was overlaid with model 2 with different fractions of monomer and dimer functions. A fraction of $42 \%$ monomers $\left(x_{0}=\right.$ 0.42 ) matches the data better compared to $x_{0}=0.5$ (Figure 4.2.5 A), which verifies that the analysis of the blinking histogram underestimates the fraction of monomers. Quantification across different linear combinations of monomers and dimers showed a 
maximal deviation at equal fractions of monomers and dimers (Figure 4.2.5 B). In addition, the deviation of monomer and dimer fractions was investigated at different assumed detection efficiencies. The investigation showed that small detection efficiencies lead to larger deviations (Figure 4.2.5 C). Knowing the exact detection efficiency allows the correction for these deviations (equation 20):

$y_{m}=\frac{x_{m}}{P_{d}^{(m)}}\left[\sum_{j=0}^{M} \frac{x_{j}}{P_{d}^{j}}\right]^{-1}$

The corrected fractions $\left(y_{m}\right)$ are obtained from the apparent fractions $\left(x_{m}\right)$ by taking into account the probability of detecting an oligomer with $m+1$ co-localized fluorescent proteins $\left(P_{d}^{(m)}\right)$ and the term $\left[\sum_{j=0}^{M} \frac{x_{j}}{P_{d}^{j}}\right]^{-1}$ for normalization of the fractions by considering undetected oligomers. This allows accurate determination of different types of oligomers from PALM data and extends the existing method towards oligomers that are not detected. Sebastian Malkusch, Jakob Bullerjahn and Gerhard Hummer were mainly responsible for the generalization, for example of corrections such as equation 20 .
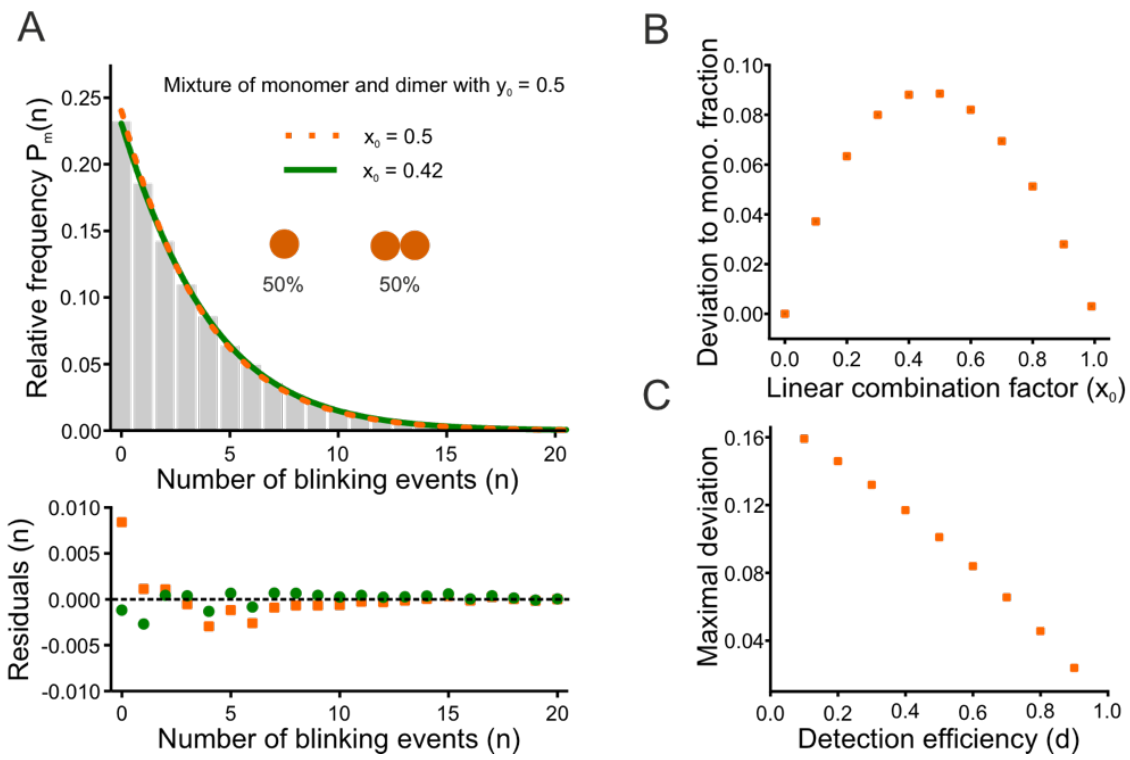

Figure 4.2.5: Deviations in the analysis of qPALM data due to undetected oligomers. (A) Histogram of the relative frequency of the number of blinking events for simulated qPALM data of monomers and dimers at equal fractions. Two functions of model 2 with different linear combination factors $\left(x_{0}\right)$ are shown as orange dashed line $\left(x_{0}=0.5\right)$ and green solid line $\left(x_{0}=\right.$ 0.42). Residuals of both functions are shown at the bottom in the respective color. (B) Deviation of the obtained monomer fraction from the ground truth for different linear combination factors. (C) Maximal deviations $\left(x_{0}=0.5\right)$ of obtained monomer fractions to the ground truth monomer 68 
fraction for different detection efficiencies. Adapted from Baldering et al (2019a). Copyright CC BY 4.0.

\subsubsection{Accurate determination of mixtures of higher oligomers}

In some biological samples, the protein of interest might be populating three different types of oligomeric states. Hence, an accurate determination of the respective fractions is necessary. Mixtures of oligomers with a complexity of up to trimers were simulated. The histogram was well approximated by a combination of monomer, dimer, and trimer functions and apparent fractions $\left(x_{0}, x_{1}, x_{2}\right)$ were obtained from the approximation (Figure 4.2.6).

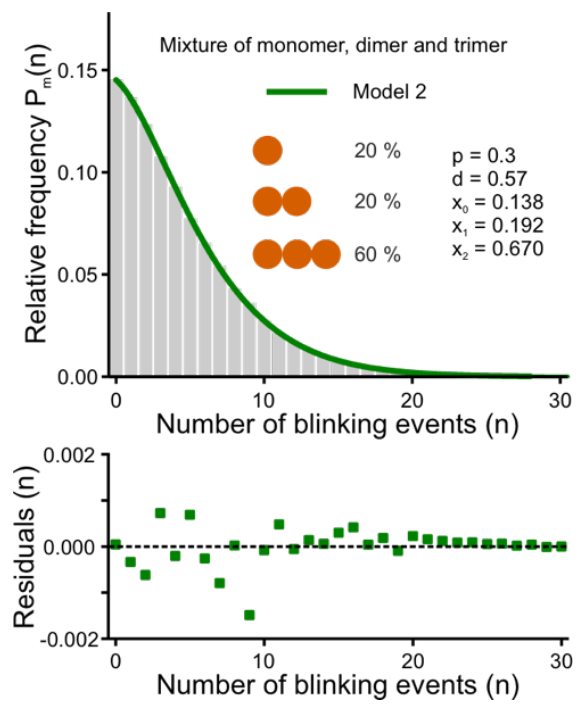

Figure 4.2.6: Accurate determination of multiple oligomers with complexity $\boldsymbol{m} \leq \mathbf{2}$. Histogram of the relative frequency of the number of blinking events for simulated qPALM data of monomers, dimers, and trimers. Monomer, dimer and trimer fractions are $20 \%, 20 \%$, and $60 \%$, respectively. Apparent fractions obtained by fitting are given for monomers $\left(x_{0}=0.138\right)$, dimers $\left(x_{0}=0.192\right)$, and trimers $\left(x_{0}=0.670\right)$. Residuals are shown at the bottom. Adapted from Baldering et al (2019a). Copyright CC BY 4.0.

These apparent fractions showed expected deviations from the ground truth due to undetected oligomers (Figure 4.2.6). By applying model 2, fractions were obtained that match the ground truth values very well (Table 4.2.2). The deviations for the determined monomers to the ground truth decreased from up to $31 \%$ for the uncorrected fractions to $4 \%$ for the corrected fraction. This demonstrates the higher accuracy of the revised model by including a correction term that allows the consideration of undetected oligomers. 
Table 4.2.2: Accurate determination of mixtures of oligomers from qPALM data with model

2. Simulations of monomers, dimers, and trimers were performed with the ground truth fractions. Apparent fractions were obtained from approximating the histogram of the number of blinking events with monomer, dimer, and trimer functions weighted by the respective fractions. Corrected fractions of monomers, dimer, and trimers were determined with equation 20.

\begin{tabular}{lccc}
\hline $\begin{array}{l}\text { Oligomer } \\
\text { fraction }\end{array}$ & Ground truth fraction & $\begin{array}{l}\text { Apparent } \\
\text { fraction }\end{array}$ & $\begin{array}{l}\text { Corrected } \\
\text { fraction }\end{array}$ \\
\hline Monomer & 0.2 & $0.138 \pm 0.06$ & $0.192 \pm 0.08$ \\
Dimer & 0.2 & $0.192 \pm 0.13$ & $0.218 \pm 0.13$ \\
Trimer & 0.6 & $0.670 \pm 0.07$ & $0.590 \pm 0.06$ \\
\hline
\end{tabular}

\subsubsection{Simulated qPALM data of oligomers using mEos3.2 blinking statistics}

Since model 2 was able to describe the simulations very well, the next step was to generate artificial oligomers from mEos3.2 qPALM data (Figure 4.2.7). Higher-ordered oligomers were produced by grouping two, three, or four single mEos3.2 molecules and summing up the blinking events into histograms (Figure 4.2.7 A). These histograms were very well approximated with $p=0.32$ and $d=1$. Next, dimers and trimers were simulated by considering a detection efficiency of $d=0.57$, which is close to real experimental data and reflects the detection efficiency of mEos3.2 determined from dimeric mEos3.2 (Figure 4.2.7 B, C). The fractions of ideal dimers/trimers and defective dimers/trimers were calculated, normalized after completely active oligomers, and finally used to generate the histogram of blinking events. The detection efficiency was calculated from the approximation and a small error of 0.02 was found compared to the ground truth. Finally, an equal mixture of monomers and dimers was generated from the mEos3.2 qPALM data (Figure 4.2.7 D). The fractions of active fluorophores in the respective oligomers were calculated according to the detection efficiency and used for the blinking histogram analysis. The apparent fractions were determined by approximation, and the correction analysis yielded fractions of monomers and dimers that almost perfectly matched the ground truth (Figure 4.2.7 E). 
A
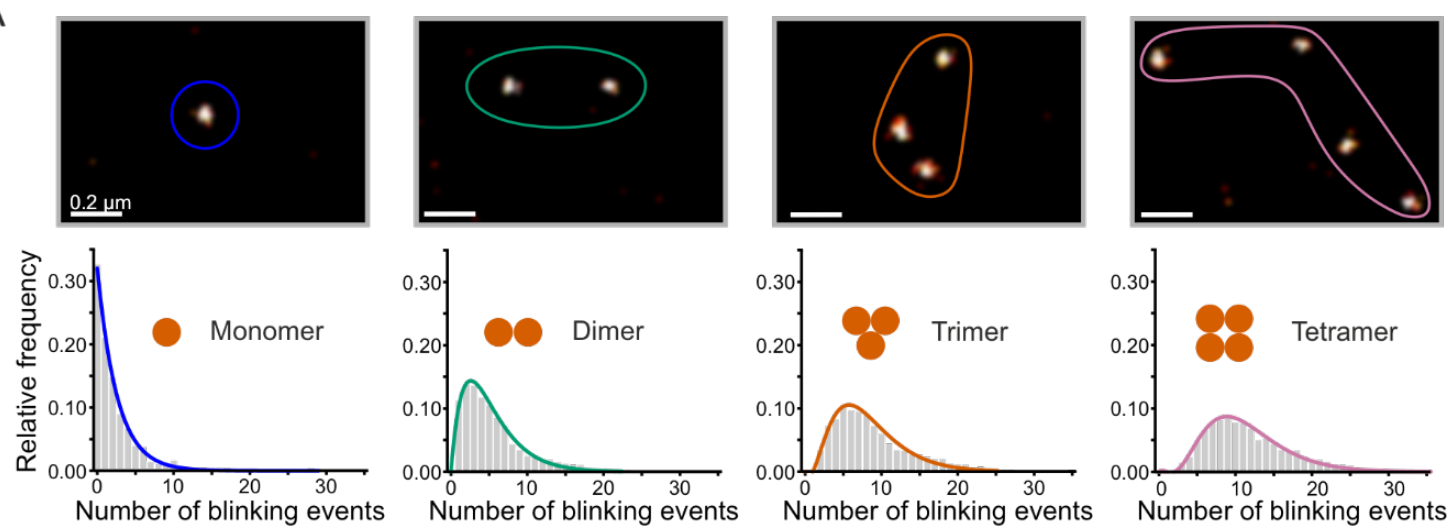

\section{B}

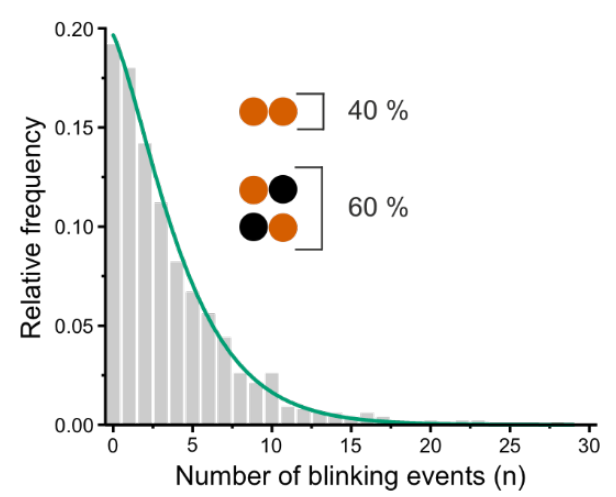

D

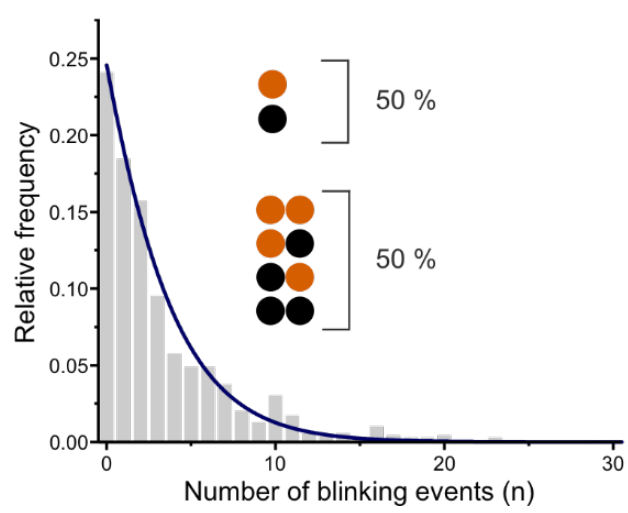

C

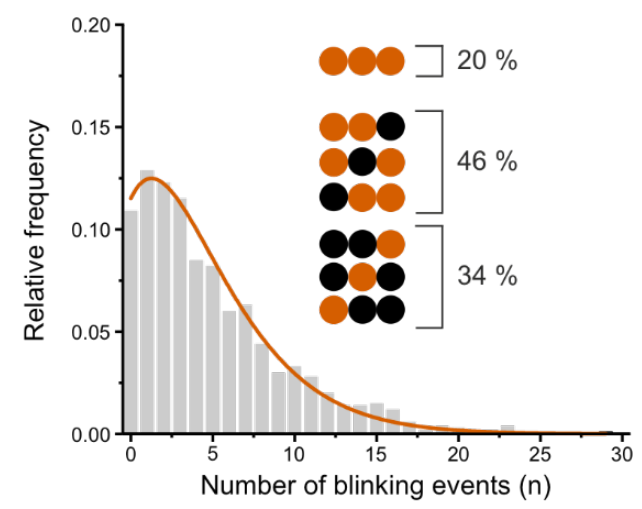

E

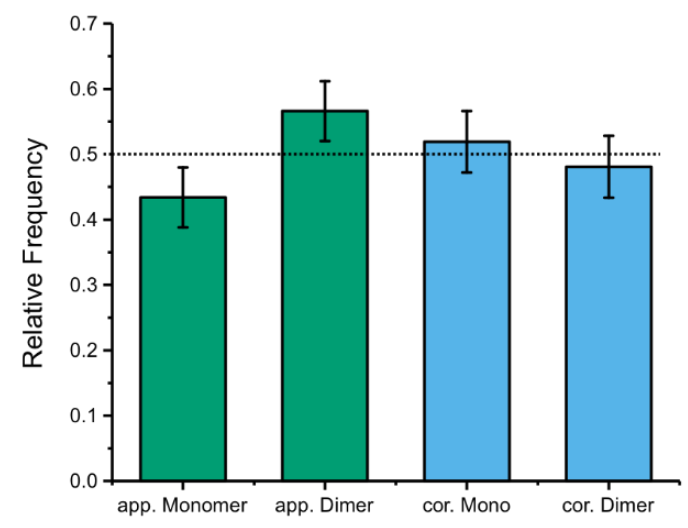

Figure 4.2.7: Simulations of oligomers from single-molecule PALM data of mEos3.2. (A)

qPALM data of oligomers was simulated by grouping single-molecule localizations of mEos3.2 into dimers (green), trimers (orange), and tetramers (purple). The histograms of the relative frequency of the number of blinking events were generated and approximated with the function of monomers (blue), dimers (green), trimers (orange), and tetramers (purple) with $p=0.32$ and $d=1$. (B, C) Histograms of blinking events from artificial dimers (B) and trimers (C) assuming a detection efficiency of $d=0.57$. The respective fractions depicted in the figure are normalized values that reflect the input of the simulation. The histograms were approximated by a dimer (green) or trimer (orange) function, respectively, with $p=0.32$ and $d$ being determined from the approximation. (D) Histograms of blinking events from a 1:1 mixture of monomers and artificial dimers assuming a detection efficiency of $d=0.57$. Total oligomers having one or two active 


\section{Results \& Discussion}

fluorophores were calculated and used for generating the blinking event distribution. A linear combination of monomer and dimer fit functions was used with $p=0.32, d=0.57$ and the apparent fractions were determined. (E) Apparent fractions from (D) were corrected with equation 20 and corrected monomer and dimer fractions were obtained. The dotted black line indicates the ground truth. Adapted from Baldering et al (2019a). Copyright CC BY 4.0.

\subsubsection{Discussion}

This chapter demonstrates that detection efficiency is a key parameter in the analysis of protein oligomeric states with qPALM. Other methods such as qPAINT (Jungmann et al. 2016) or the pair-correlation analysis (Sengupta et al. 2011) do not yet consider the detection efficiency, which leads to an underestimation of the oligomeric state. In contrast, qPALM accounts for inactive fluorophores in oligomers, but the originally introduced $q$-value is limited to report the inactive fluorescent proteins in a dimer. The revised model presented here introduces a new parameter, the detection efficiency $d$, which accounts for all inactive fluorescent proteins and allows the accurate description of higher-ordered oligomers. In addition, a modified analysis is reported which corrects for oligomers that remain undetected.

\section{Influence of the new findings of qPALM theory on experimental data}

In previous studies, the original analysis model (Hummer et al. 2016) was used, which underestimated undetected monomers. However, this effect is small for monomer/dimer mixtures at high detection efficiencies. For example, the monomer and dimer fractions of the Toll-like receptor 4 (TLR4) were investigated (Krüger et al. 2017) and dimerization of TLR 4 was increased from $48 \%$ dimers to $73 / 74 \%$ dimers by stimulating the cells with lipopolysaccharides, LPS $_{\mathrm{EC}}$ and $\mathrm{LPS}_{\mathrm{SM}}$. The fraction of monomers may have been slightly underestimated to about $1-5 \%$, which does not affect the results significantly as a detection efficiency of about 0.8 was determined and applied (Figure 4.2.5 C). Another study reported on photobleaching experiments of TLR4-GFP in which lower fractions of dimers (12.5\%, 52\% stimulated with LPS) were reported (Yang et al. 2014). This would coincide with the fact that the monomer fractions in Krüger et al 2017 were slightly underestimated although the effect is marginal. Monomer and dimer fractions were also studied in this thesis (Section 4.1.2; 4.1.3). Using the original model, a higher fraction of monomers in the range of $1-5 \%$ is expected than was determined from the qPALM experiments (Figure 4.1.7 C). Indeed, the monomer fraction changed from $18 \%$ to $21 \%$ 
after the correction for undetected oligomers was applied for the qPALM analysis of the genetic dimer. This slightly higher fraction of monomers fits better to the estimates from the SDS gel of the genetic dimer, although the intensity analysis of the gel is not that accurate. In summary, this shows that the deviations are small and should not affect the main conclusions reported.

In Karathanasis et al. (2020), the oligomeric state of the tumor necrosis factor (TNF) receptor was analyzed (Karathanasis et al. 2020) using the original model (Hummer et al. 2016). This study reported an arrangement of TNF receptors into trimers and nonamers upon stimulation with the natural ligand TNF $\alpha$. The $q$-value was used for the approximation for a mixture of trimers and nonamers. Undetected oligomers were not considered. Considering these deviations, a slightly lower fraction of trimers and nonamers, and a higher fraction of monomers is expected when using the revised model for qPALM analysis. However, these variations should only affect the fractions of oligomers and not the model complexity and thus not the existence of the different types of oligomers.

The limitations of qPALM are mainly related to the time separation of single blinking events. Low intensity of violet light would decrease the probability of activation, hence decreasing the number of overlapping signals. However, at a distinct order of oligomers, the simultaneous emission of single fluorophores in an oligomer would lead to an underestimation. This limit has to be estimated practically with calibration proteins of higher order.

In summary, the revised model is more suitable in describing the blinking data of higherordered oligomers such as trimers, tetramers, hexamers, and nonamers than the previously used model. Furthermore, undetected oligomers can be considered by the detection efficiency which, if corrected for, led to very precise determinations of oligomeric fractions in simulations. The deviations between model 1 and 2 are small if oligomers of lower orders are analyzed and a high detection efficiency is used. Therefore, the obtained oligomer fractions in previous studies should be sparsely affected (Krüger et al. 2017; Baldering et al. 2019b; Karathanasis et al. 2020). Nevertheless, the use of the revised model is recommended in future studies to obtain precise oligomer fractions. 


\subsection{CRISPR/Cas12a-assisted endogenous tagging of membrane receptors for super-resolution microscopy}

Single-molecule localization microscopy enables the specific detection of target proteins at near-molecular resolution. In combination with stoichiometric protein labeling and quantitative single-molecule analysis, the molecular assembly of proteins becomes accessible. Fluorescent proteins are very well suited for quantitative approaches as they label the target protein stoichiometrically, and several methods exist for generating fusion proteins of fluorescent proteins. One method is the generation of plasmids that code for the DNA of the fusion protein and that can be transfected into e.g. mammalian cells. However, this method can lead to unphysiological expression levels of the target protein which might alter reaction kinetics and the organization of a protein in a cell (Lisenbee et al. 2003; Doyon et al. 2011; Gibson et al. 2013). At the same time, large variations in expression level between cells occur, because of a different uptake of a plasmid. Additionally, knockdowns are required because otherwise the wildtype protein may interact with the fusion protein, which might complicate analysis. If the protein is not naturally expressed in a cell, it might not behave as in its native environment because key interacting proteins are missing, or the environment such as the composition of the membrane is different. Therefore, tools are necessary to directly engineer the genomic DNA of a target protein in a cell and to allow endogenous expression while maintaining a native environment.

Since 1993, the CRISPR/Cas technology has been developed (Mojica et al. 2005; Pourcel et al. 2005; Bolotin et al. 2005; Makarova et al. 2006; Barrangou et al. 2007; Brouns et al. 2008; Marraffini und Sontheimer 2008; Hale et al. 2009; Garneau et al. 2010; Deltcheva et al. 2011; Sapranauskas et al. 2011; Gasiunas et al. 2012; Jinek et al. 2012), which allows genetic engineering of target proteins in various cell lines (Le Cong et al. 2013; Mali et al. 2013). CRISPR/Cas allows to specifically cut and modify DNA using the Cas enzyme and crRNA. The combination of CRISPR/Cas9 with PALM was demonstrated to be very useful and effective for different applications (Ratz et al. 2015; Cho et al. 2016; Hansen et al. 2017; Khan et al. 2017; Khan et al. 2019). However, small labeling efficiencies were obtained or the production of appropriate inserts for generating stable cell lines was time intense. Recently, CRISPR/Cas12a-assisted PCR tagging has proven to be suitable for rapid generation of homology templates (PCR cassette) and is very effective and efficient due to the use of Cas12a as cutting enzyme (Figure 4.3.1). This section shows the simplicity and high efficiency of PCR tagging in combination with 
PALM (Baldering et al. 2020). Two receptor tyrosine kinases, MET and EGFR, were labeled with the fluorescent protein mEos $4 \mathrm{~b}$ using this novel labeling technology and the specific integration was verified by PCR and western blots. Furthermore, the native activity of the stable cell lines upon ligand stimulation was confirmed. For the MET receptor, almost complete labeling was achieved in HEK293T cells (approximately 81\%), while the fraction of labeled EGFR in HEK293T cells was lower (approximately 25\%). Due to its high labeling efficiency, the oligomeric state of MET was analyzed using the revised qPALM model (Section 4.2). The membrane dynamics of MET were assessed in live-cell experiments.

\subsubsection{Generation of stable cell lines via CRISPR/Cas12a-assisted genome editing (PCR tagging)}

PCR tagging is a genome editing tool that has proven time efficient and high labeling efficiencies (Fueller et al. 2020). In a first step, primers for EGFR and the MET receptor, which were necessary for the production of the PCR cassettes, were designed with the help of a web interface, www.pcr-tagging.com. The PCR cassettes were prepared in a PCR (see section 3.8 for details), applied to an agarose gel and the size of the PCR cassettes was verified (Figure 4.3.1 B). After purification, the PCR cassettes and a Cas 12a helper plasmid were cotransfected into HEK293T cells (Figure 4.3.1 C). 
A
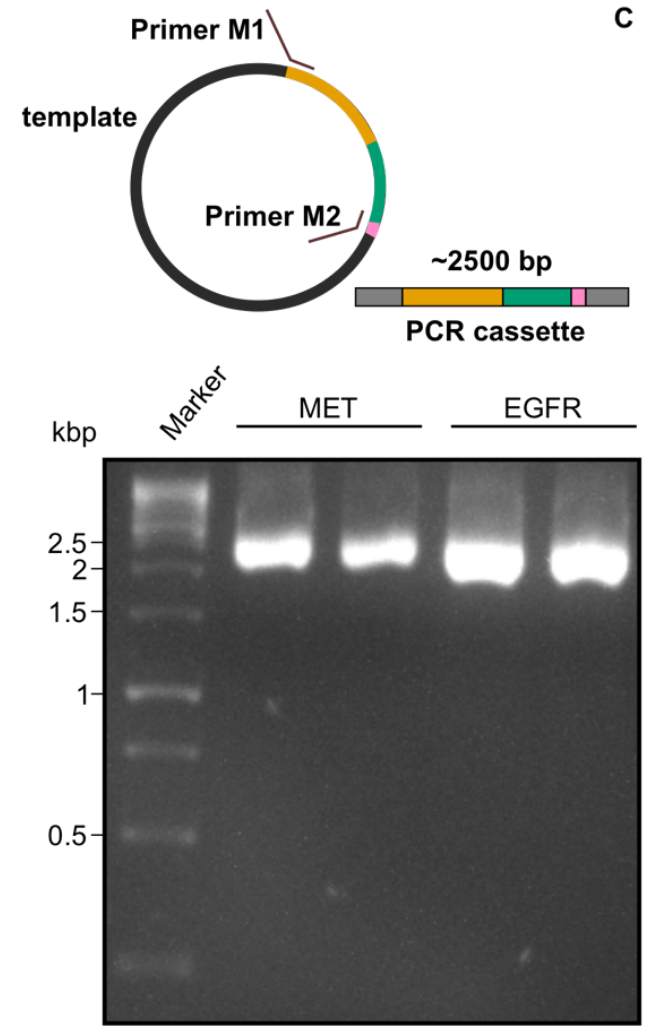

C

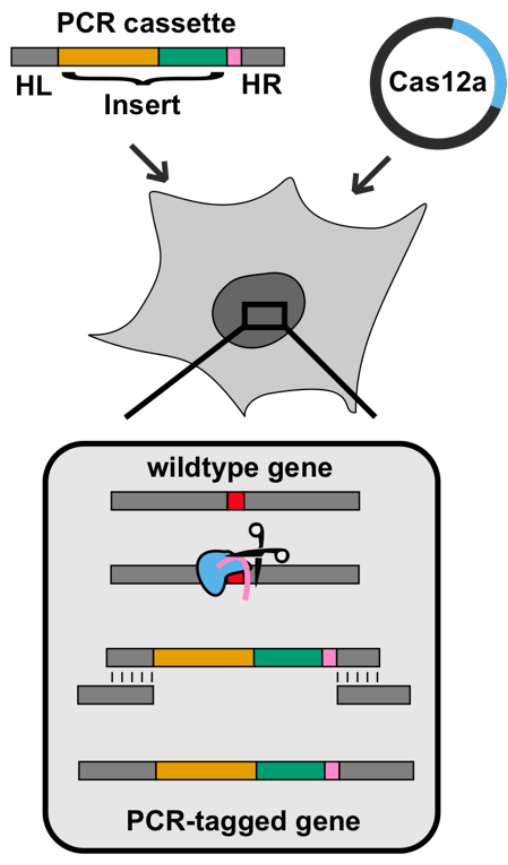

Figure 4.3.1: Generation of PCR cassettes for CRISPR/Cas12a-assisted genome editing. (A) Schematic illustration of the PCR reaction to produce the PCR cassette (yellow: fluorescent protein; green: puromycin; pink: crRNA). (B) Agarose gel showing the amplified PCR cassettes of MET and EGFR with expected sizes of 2502 and 2551 bp. (C) Schematic illustration of genome editing via CRISPR/Cas12a. The PCR cassette and the Cas12a helper plasmid are cotransfected into HEK293T cells. Cas12a (blue) is expressed, forms a complex with the crRNA (pink) and specifically cuts the DNA near the protospacer adjacent motif (PAM) sequence (red) in the genome. During homology directed repair, the PCR cassette is inserted into the genome.

Stable cell lines were generated by puromycin selection and subsequent isolation of individual cells by dilution and further analyzed by fluorescence microscopy. Approximately two thirds of the clones (10 out of 16) showed a clear fluorescence signal that was significantly different from that of the wildtype unlabeled cells (Figure 4.3.2). The fluorescence signal showed distinct punctiform membrane localizations thus suggesting the expression and localization of the mEos4b-tagged receptors in the plasma membrane. 

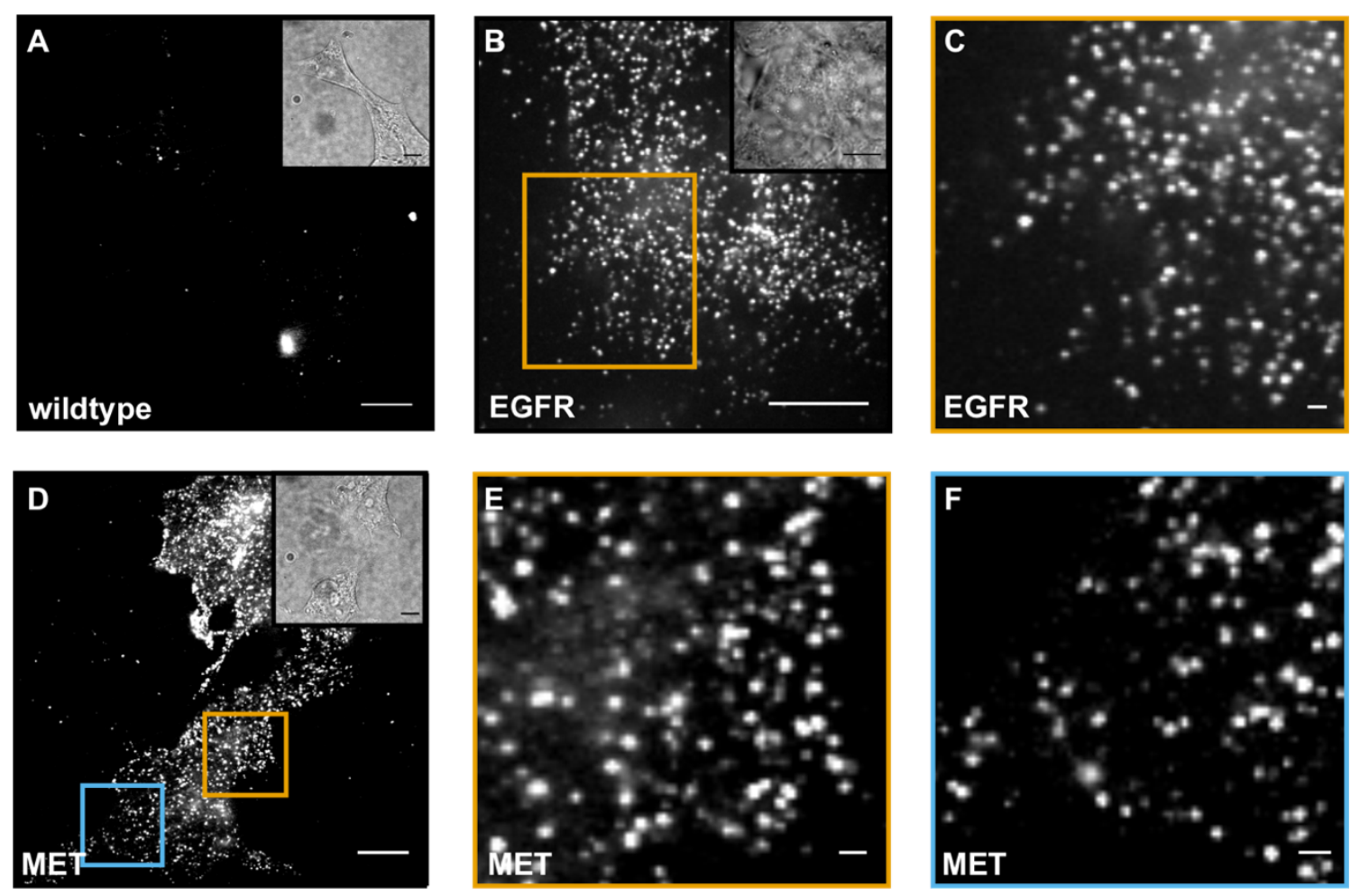

Figure 4.3.2: Fluorescence images of wildtype and CRISPR/Cas12a-modified HEK293T

cells. (A) Diffraction-limited fluorescence image of wildtype HEK293T cells showing only unspecific background fluorescence. The brightfield image is shown on the upper right. (B) Diffraction-limited fluorescence image of a HEK293T cell expressing EGFR-mEos4b. The brightfield image is shown on the upper right. (C) Zoom-in of (B). (D) Diffraction-limited fluorescence image of a HEK293T cell expressing MET-mEos4b. The brightfield image is shown on the upper right. The scale bars are $10 \mu \mathrm{m}$. (E,F) Zoom-ins of (D). Scale bars are $10 \mu \mathrm{m}$ (A,B,D) and $1 \mu \mathrm{m}(\mathrm{C}, \mathrm{E}, \mathrm{F})$. Parts adapted from Baldering et al (2020). Copyright CC BY 4.0.

Two fluorescent clones of EGFR and MET were randomly selected, and their genome was analyzed by PCR (Figure 4.3.3). Primers annealing inside and outside of the PCR cassette were used to confirm both edges of the genomic insertion (Figure 4.3.3A). Two sequences were generated which showed the expected sizes of about 1,000 and 2,300 bp, respectively (Figure 4.3.3 B, C). While MET C2 and EGFR D6 showed only one positive PCR fragment, MET C6 and EGFR F8 were positive in both PCRs. The respective DNA fragments were extracted, purified and the correct DNA sequence was confirmed by DNA sequencing. 
A

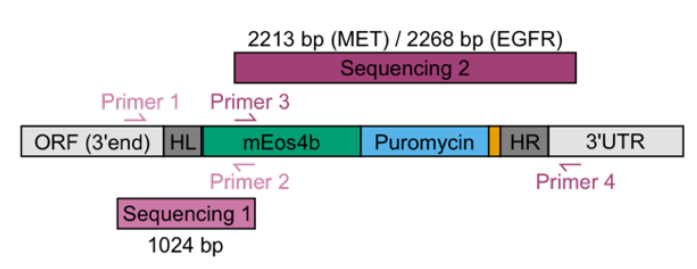

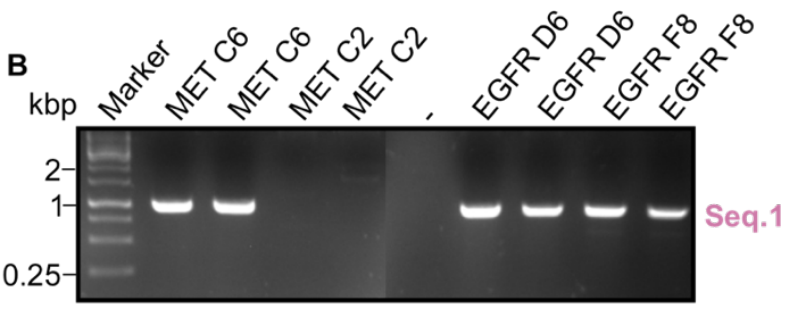

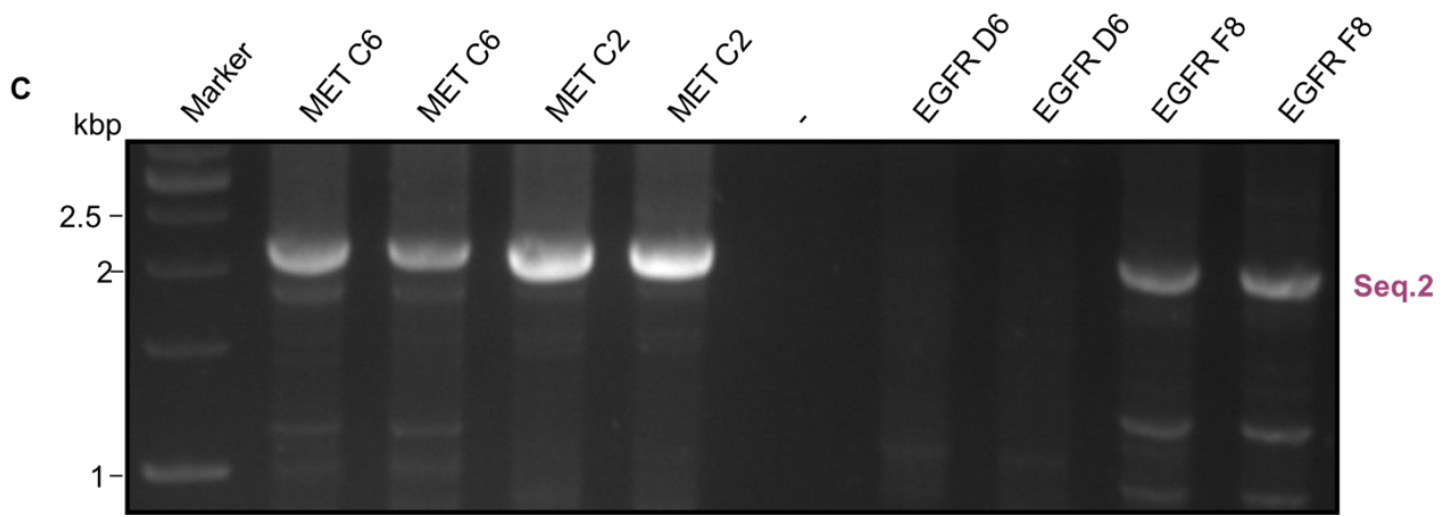

Figure 4.3.3: PCR of the edited part of the genome sequence in CRISPR/Cas12a-edited stable cell lines. (A) Schematic illustration of the genomic insertion of the PCR cassette. Primers 1-4 show the sites of generation of PCR fragments for verifying the insertion of the PCR cassette. (B) PCR with primers 1 and 2 were designed to verify the left insertion site of the PCR cassette. Two cell lines of MET (C2; C6) and EGFR (D6; F8) were analyzed. Bands at approximately $1,000 \mathrm{bp}$ show the desired fragment. (C) PCR with primers 3 and 4 were designed to verify the right insertion site of the PCR cassette. Two stable cell lines of MET (C2; C6) and EGFR (D6; F8) were analyzed. Bands at approximately $2,300 \mathrm{bp}$ show the desired fragment. Parts adapted from Baldering et al (2020). Copyright CC BY 4.0.

The cell lines MET C6 and EGFR F8 were further analyzed by western blots to confirm the correct expression of these fusion proteins and to estimate the fraction of wildtype versus genetically modified receptors (Figure 4.3.4 A). The western blot of MET C6 showed a distinct shift of the MET protein band of about $30 \mathrm{kDa}$ from $140 \mathrm{kDa}$ (wildtype MET) to $170 \mathrm{kDa}$ (MET-mEos4b) (Figure 4.3.4 B). In addition, the band intensity at 170 $\mathrm{kDa}$ was strongly increased compared to the band at $140 \mathrm{kDa}$. An estimate of the fraction of genetically modified receptors by comparing the intensities of both bands revealed that about $81 \%$ of MET are labeled with mEos $4 \mathrm{~b}$ and $19 \%$ are wildtype receptors. The stable cell line of EGFR-mEos4b (EGFR F8) also showed a clear shift in protein size, but with a weaker intensity (Figure 4.3.4 C). The analysis of band intensities estimated that roughly $25 \%$ of EGFR are labeled with mEos $4 \mathrm{~b}$. The main fraction of EGFR (75\%) showed the size of the wildtype receptor. 
A

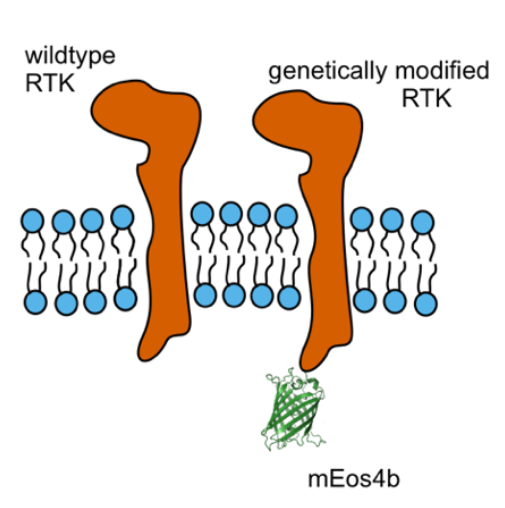

B

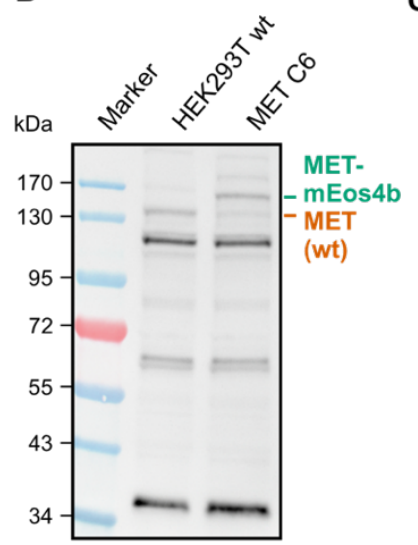

C

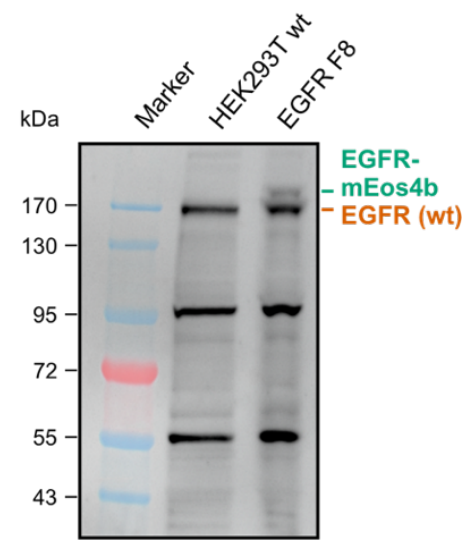

Figure 4.3.4: Western blots of MET-mEos4b and EGFR-mEos4b. (A) Schematic illustration of the protein structure of the wildtype and genetically modified receptor tyrosine kinases (RTKs). The RTK is shown in orange and the genetic modification, here mEos $4 b$, is depicted in green (crystal structure of mEos2; PDB: 3S05). (B) Western blot of HEK293T wildtype and genetically modified cells (MET C6). An antibody against the MET receptor was used. The bands of fulllength MET and MET fusion proteins are labeled in orange and green, respectively. (C) Western blot of HEK293T wildtype and genetically modified cells (EGFR F8). An antibody against the EGF receptor was used. Bands of full-length EGFR and EGFR fusion proteins are marked. Parts adapted from Baldering et al (2020). Copyright CC BY 4.0.

The stable cell lines were functionally analyzed to confirm the activity of both receptors. In a first step, the phosphorylation of the receptors was investigated. The MET C6 cell line showed a clear response to $\mathrm{HGF}$ and $\mathrm{InlB}_{321}$-ATTO $647 \mathrm{~N}$ similar to the wildtype HEK293T cells, while without the addition of HGF no or only very weak bands of phosphorylated MET were detected (Figure 4.3.5 A). Phosphorylated MET was found in MET C6 cells at protein sizes of 140 and $170 \mathrm{kDa}$, corresponding to both wildtype MET and MET-mEos4b. The comparison of these band intensities showed a similar amount of phosphorylated MET-mEos4b (83\%) compared to phosphorylated wildtype MET (Figure 4.3.4 B, figure 4.3.5 A).

Without EGF treatment, no phosphorylation of EGF receptors was detected. Phosphorylation of EGFR was detected in both wildtype and genetically modified cells upon ligand stimulation with EGF or EGF-CF640 (Figure 4.3.5 B). However, antibody staining resulted in blurred bands, which precluded a clear distinction between labeled and unlabeled EGFR. 
Since phosphorylation of the receptors alone may not be sufficient to initiate signal transduction (Liang et al. 2018), western blots were performed to analyze phosphorylation of MAPK to prove downstream signaling. Ligand stimulation of wildtype HEK293T cells with HGF and EGF led to phosphorylation of MAPK, whereas without ligand treatment only weak bands were detected (Figure 4.3.5 C). Similarly, the MET C6 cell line showed strong phosphorylation upon treatment with HGF and InlB $321^{-}$ ATTO 647N. Treatment with EGF and EGF-CF640 also led to phosphorylation of MAPK in EGFR F8 cells. However, since the main fraction of EGFR is wildtype in the EGFR F8 cells, no clear statement can be drawn on whether the EGFR-mEos4b fusion is fully active.

A

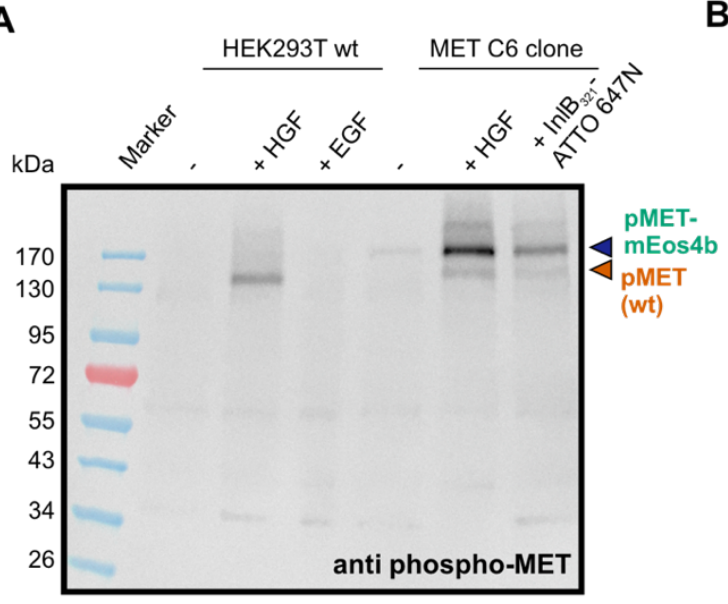

$B$

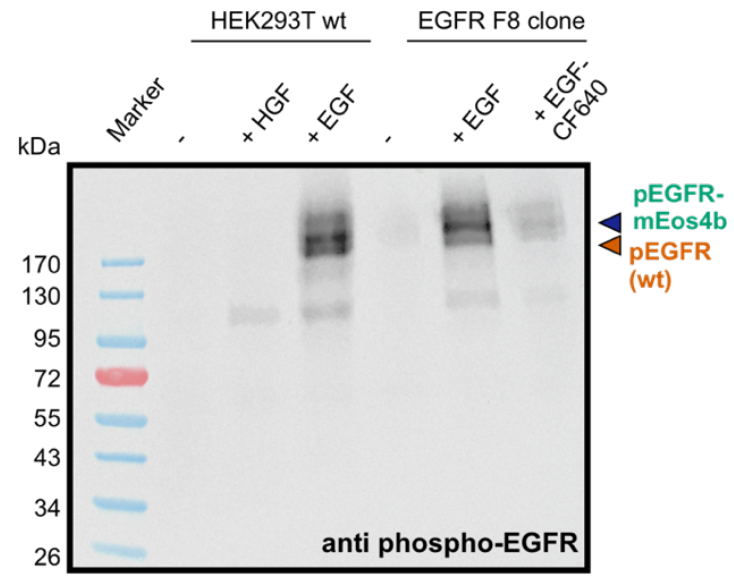

C

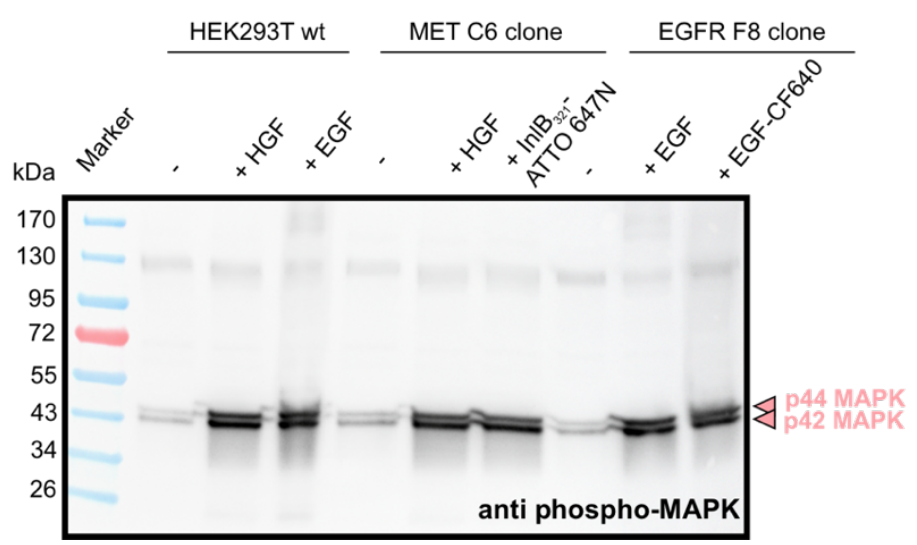

Figure 4.3.5: Western blots of receptor and MAPK phosphorylation to verify native signal transduction of MET-mEos4b and EGFR-mEos4b. (A) Western blot of wildtype and genetically modified HEK293T cells (MET C6). An antibody against phosphorylated MET receptors was used. Cells were stimulated by different ligands (HGF, EGF, and InlB 321 -ATTO $647 \mathrm{~N}$ ). Colored arrows mark the bands of full-length proteins. (B) Western blot of wildtype and genetically modified HEK293T cells (EGFR F8). An antibody against phosphorylated EGFR was 
used. Cells were stimulated by different ligands (HGF, EGF, and EGF-CF640). Colored arrows mark the bands of full-length proteins. (C) Western blot of wildtype and genetically modified HEK293T cells (MET C6; EGFR F8). An antibody against phosphorylated MAPK was used. Cells were stimulated by different ligands (HGF, InlB 321 -ATTO 647N, EGF, and EGF-CF640). Colored arrows mark the bands of full-length proteins. The marker bands allow the size of the respective protein to be estimated. Parts adapted from Baldering et al (2020). Copyright CC BY 4.0 .

These experiments prove that the MET C6 cell line is functional and contains a stoichiometric fluorescent protein label of MET. This allows studying the MET receptor in its native environment as it contains nearly completely labeled receptors that are phosphorylated upon ligand stimulation and most likely initiate native signal transduction. In contrast, the EGFR cell line showed incomplete labeling of the receptor and therefore activity was difficult to prove as the main fraction of EGFR was wildtype. In summary, CRISPR/Cas12a-assisted protein tagging is a powerful tool for PALM.

\subsubsection{Analysis of the oligomerization state of the MET receptor}

Unperturbed quantification of protein oligomerization requires a native expression level, homogenous expression, and a stoichiometric and efficient labeling. Furthermore, a high localization precision is necessary for resolving single receptors or receptor clusters. In order to demonstrate that these requirements are met with the cell line MET C6, the localization precision of MET-mEos $4 b$ was determined. A NeNa value of $10 \pm 3 \mathrm{~nm}$ was obtained from ten cells and confirmed a near-molecular resolution. Furthermore, cellto-cell heterogeneity was analyzed by determining the number of receptor sites (unresolved "spots" of MET) per $\mu \mathrm{m}^{2}$. From ten cells, an average of $0.63 \pm 0.32$ receptor clusters $/ \mu \mathrm{m}^{2}$ were obtained, thus confirming both native and homogeneous expression of MET-mEos4b between individual cells. Therefore, MET C6 cells provide a prime platform to analyze the MET receptor in its native environment.

PCR tagging of the MET receptor resulted in stoichiometric labeling of MET with a high labeling efficiency, which facilitates quantitative analysis of the oligomeric state of MET. qPALM was selected for quantification, which has been further improved both practically and theoretically in this thesis (Sections 4.1 and 4.2). Two parameters are essential for this analysis, the bleaching probability $(p)$, which describes the blinking characteristics of the fluorophore, and the detection efficiency $(d)$. In section 4.1.3, the parameters $p$ and 
$d$ were determined in HEK293T cells with CD86-mEos4b and CTLA-4-mEos4b. Therefore, these two parameters of the fusion proteins ( $p=0.27 ; d=0.79$ ) were used to quantify MET-mEos4b in HEK293T cells. Since MET-mEos4b has a labeling efficiency of about $81 \%$, a corrected detection efficiency of $d=0.64$ was used in qSMLM analysis.

qPALM measurements of MET C6 cells were performed in the absence and presence of HGF (Figure 4.3.6 A, B). From the PALM images, single-molecule clusters were selected from which the number of blinking events was extracted (for details see section 3.18). The determination of the blinking events of single receptor clusters of all conditions presented in this section was performed by Christos Karathanasis. The relative frequency of the number of blinking events was calculated and the histogram was analyzed by approximating the histogram with a qSMLM software written by Sebastian Malkusch (see section 3.18) (Baldering et al. 2019a) (Figure 4.3.6 C, D). In the resting state, METmEos4b appeared mainly as monomer (95 $\pm 1 \%$ monomers), while ligand stimulation with the native ligand HGF resulted in $71 \pm 3 \%$ dimers (Figure 4.3.6 E, F). This shows a clear shift towards dimeric receptors in HGF-stimulated HEK293T cells.

A
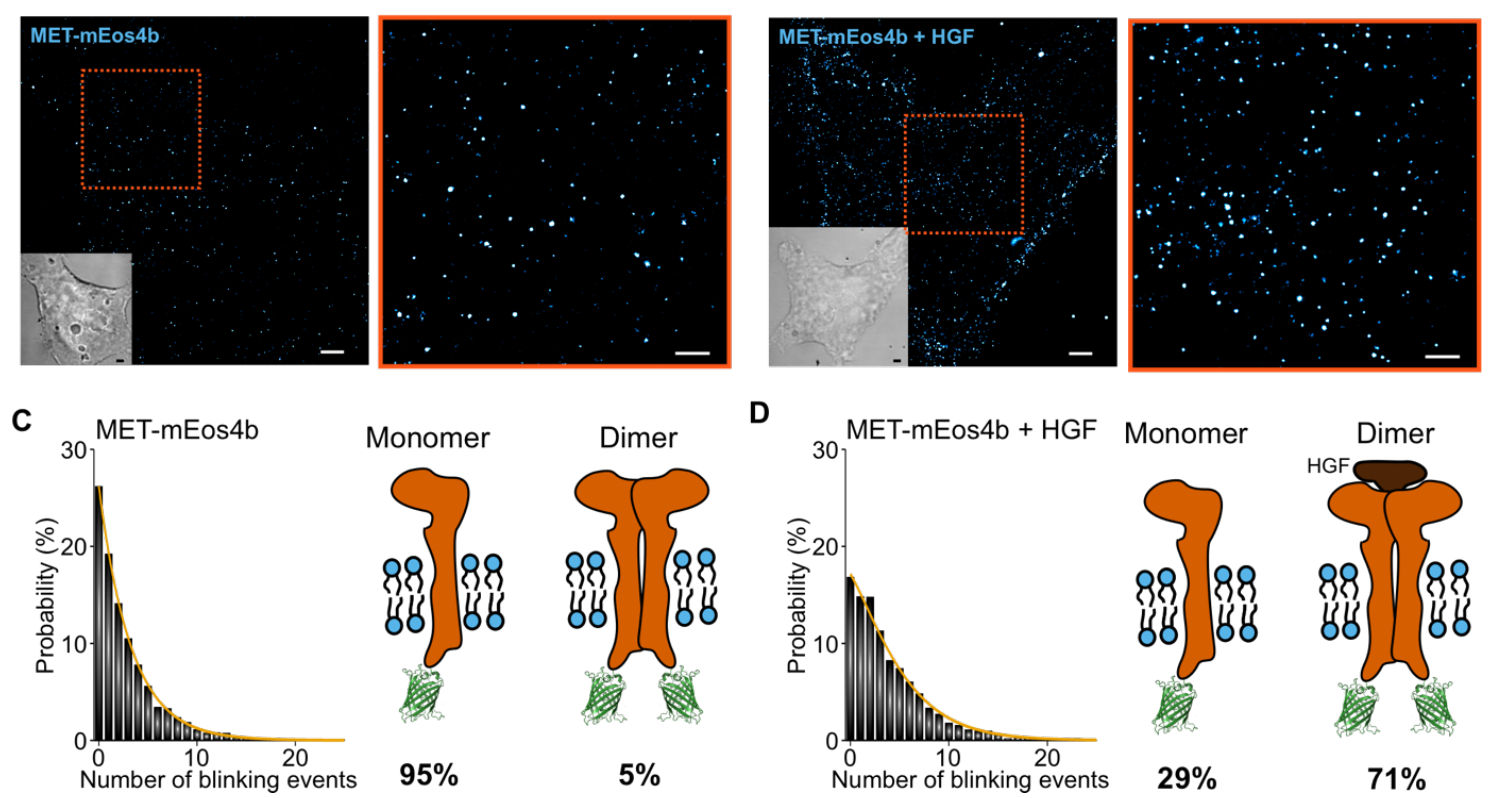

Figure 4.3.6: qPALM of MET-mEos4b in resting and HGF-stimulated HEK293T cells.

PALM image of MET-mEos4b in a resting cell (A) and HGF-stimulated cell (B). Zoom-ins are shown in the red box. A brightfield image is shown on the bottom left. Scale bars are 2 and $1 \mu \mathrm{m}$, respectively. (C, D) Histograms of the relative frequency of the number of blinking events for resting $(C)$ and HGF-stimulated (D) cells. The orange fit estimates the fraction of monomers and 
dimers. A p-value of 0.27 and a detection efficiency of $64 \%$ were used (left). Schematic illustration of the monomer-dimer ratios for resting (C) and HGF-stimulated (D) cells (right). The crystal structure of mEos2 is shown in green (PDB:3S05). Adapted from Baldering et al (2020). Copyright CC BY 4.0.

The dimerization of MET-mEos $4 b$ cells treated with the bacterial ligand InlB 321 -ATTO 647N was measured (Figure 4.3.7 A). Two analysis of MET-mEos4b were performed with respect to the colocalization of MET-mEos $4 b$ with the bacterial ligand. First, the analysis of all clusters independent of colocalization to InlB $_{321}$-ATTO $647 \mathrm{~N}$ yielded $63 \pm 2 \%$ dimers (Figure 4.3.7 B, D). The dimer fraction increased only slightly to $68 \pm$ $4 \%$ when only colocalized clusters were analyzed (Yellow arrows in figure 4.3.7 A, figure 4.3.7 C, E).

In summary, both ligands, HGF and InlB $321-A T T O 647 \mathrm{~N}$, led to an increased formation of MET dimers and thus confirm the ligand-dependent dimerization of this receptor tyrosine kinase. This demonstrates PCR tagging in combination with qPALM as a powerful tool to determine protein numbers in nanoscale clusters at endogenous expression levels.
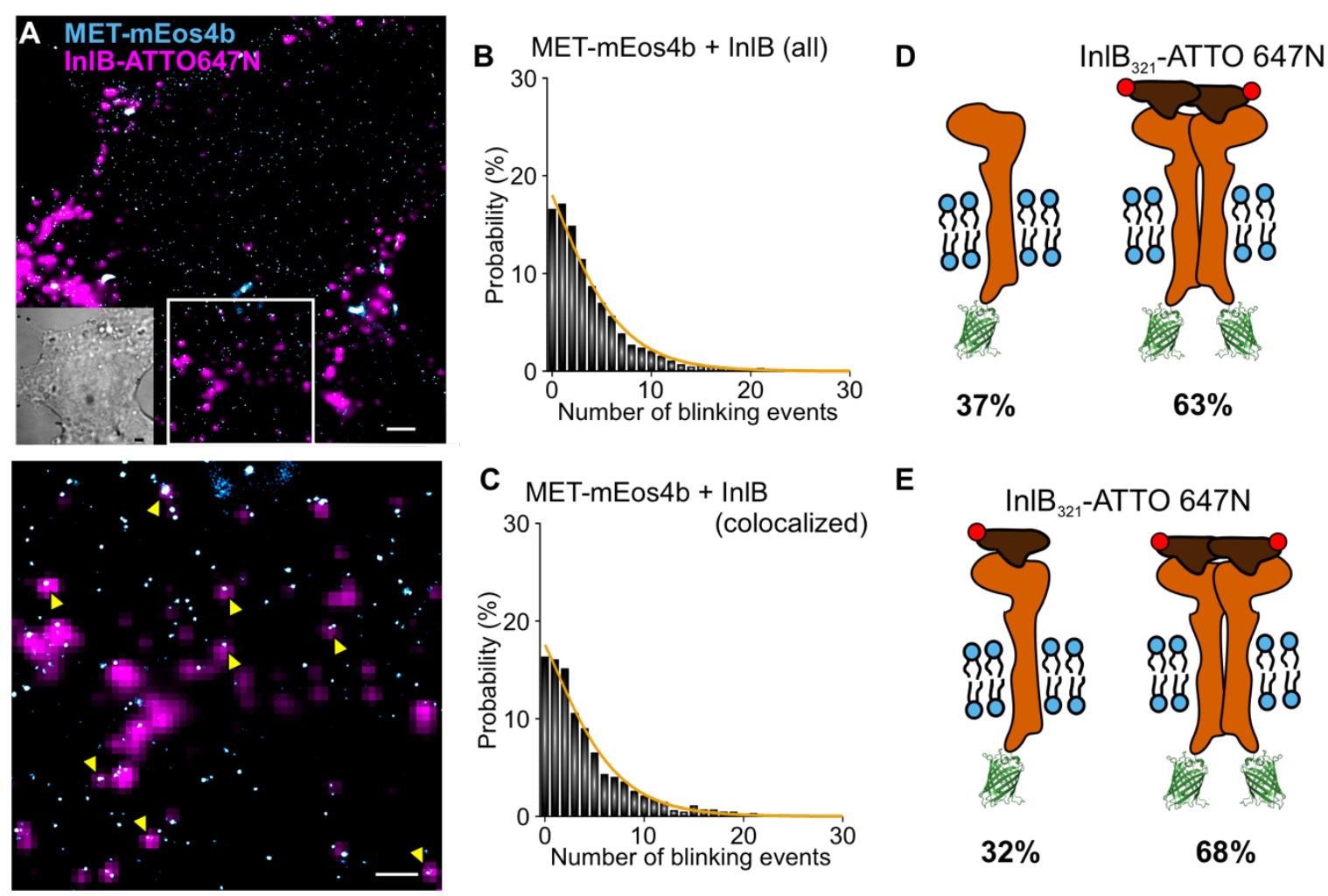

Figure 4.3.7: Quantitative PALM of InIB ${ }_{321}$-ATTO 647N-stimulated MET-mEos4b cells. (A) PALM image of MET-mEos4b (cyan) overlaid with the diffraction-limited signal of InlB $_{321}$ - 
ATTO $647 \mathrm{~N}$ (purple). A brightfield image is shown on the bottom left. The zoom-in shows colocalized spots (yellow arrows) of MET-mEos4b and InlB 321 -ATTO 647N. (B, C) Histograms of the relative frequency of the number of blinking events for all receptors in InlB 321 -ATTO 647Nactivated cells (B) and for only colocalized receptors (C). The orange fit estimates the fraction of monomers and dimers. A p-value of 0.27 and a detection efficiency of $64 \%$ were used. (D, E) Schematic illustration of the monomer-dimer ratios for all receptors (D) and for only colocalized receptors (E) obtained from the qPALM analysis. InlB $_{321}$ is illustrated in brown and ATTO 647N is shown in red. The crystal structure of mEos2 is shown in green (PDB:3S05). Scale bars are 2 $\mu \mathrm{m}$ (top) and $1 \mu \mathrm{m}$ (bottom). Adapted from Baldering et al (2020). Copyright CC BY 4.0.

\subsubsection{Analysis of the dynamics of the MET receptor}

Receptor tyrosine kinases are assumed to slow down and immobilize after ligandstimulation, because of their interaction with membrane and adaptor proteins that finally lead to the endocytosis of the receptors (Clarke und Martin-Fernandez 2019). Using the MET-mEos4b cell line, single-particle tracking PALM (sptPALM) (Manley et al. 2008) of MET-mEos $4 \mathrm{~b}$ with and without the stimulation of HGF and InlB ${ }_{321}$-ATTO $647 \mathrm{~N}$ was conducted at ambient temperature with an exposure time of $20 \mathrm{~ms}$ (for more details see section 3.19-21). Trajectories were recorded in resting cells, which mostly showed freely diffusing proteins (Figure 4.3.8 A). In contrast, free diffusion in HGF-stimulated cells was reduced and immobile spots became visible (Figure 4.3.8 B). Next, an MSD-based analysis was performed to distinguish immobile, confined, and freely diffusing proteins following a published procedure (Rossier et al. 2012). This analysis was performed by Marie-Lena Harwardt. The smallest detectable diffusion coefficient was calculated to $0.008 \mu \mathrm{m}^{2} / \mathrm{s}$ based on the dynamic localization precision (see chapter 2.7 and section 3.21). The immobile fraction in resting cells was $15 \pm 2 \%$ and increased to $72 \pm 3 \%$ and $27 \pm 3 \%$ by stimulating the cells with HGF and InlB $_{321}$-ATTO $647 \mathrm{~N}$, respectively (Figure 4.3.8 C). While free diffusion decreased substantially in HGF-stimulated cells, treatment with InlB $_{321}$-ATTO $647 \mathrm{~N}$ showed a similar fraction of freely diffusing METmEos $4 b$ as in resting cells. The fraction of confined MET-mEos $4 b$ was clearly reduced in both HGF- and InlB $321-A T T O 647 \mathrm{~N}$-stimulated cells. The MSD analysis also enabled to determine the diffusion coefficient of the individual diffusion states. For both ligandstimulated conditions, the diffusion coefficient of freely and confined diffusing particles were reduced, which was more pronounced for HGF. The diffusion coefficient of freely diffusing proteins decreased from $0.25 \pm 0.02 \mu \mathrm{m}^{2} / \mathrm{s}$ in the resting state to $0.17 \pm 0.07$ $\mu \mathrm{m}^{2} / \mathrm{s}$ in HGF-stimulated cells. The confined diffusion coefficient was even more reduced 
from $0.16 \pm 0.02 \mu \mathrm{m}^{2} / \mathrm{s}$ in resting cells to $0.05 \pm 0.02 \mu \mathrm{m}^{2} / \mathrm{s}$ in HGF-stimulated cells. This demonstrates a slowdown and immobilization of the MET receptor upon ligand stimulation with HGF and $\mathrm{InlB}_{321}$.
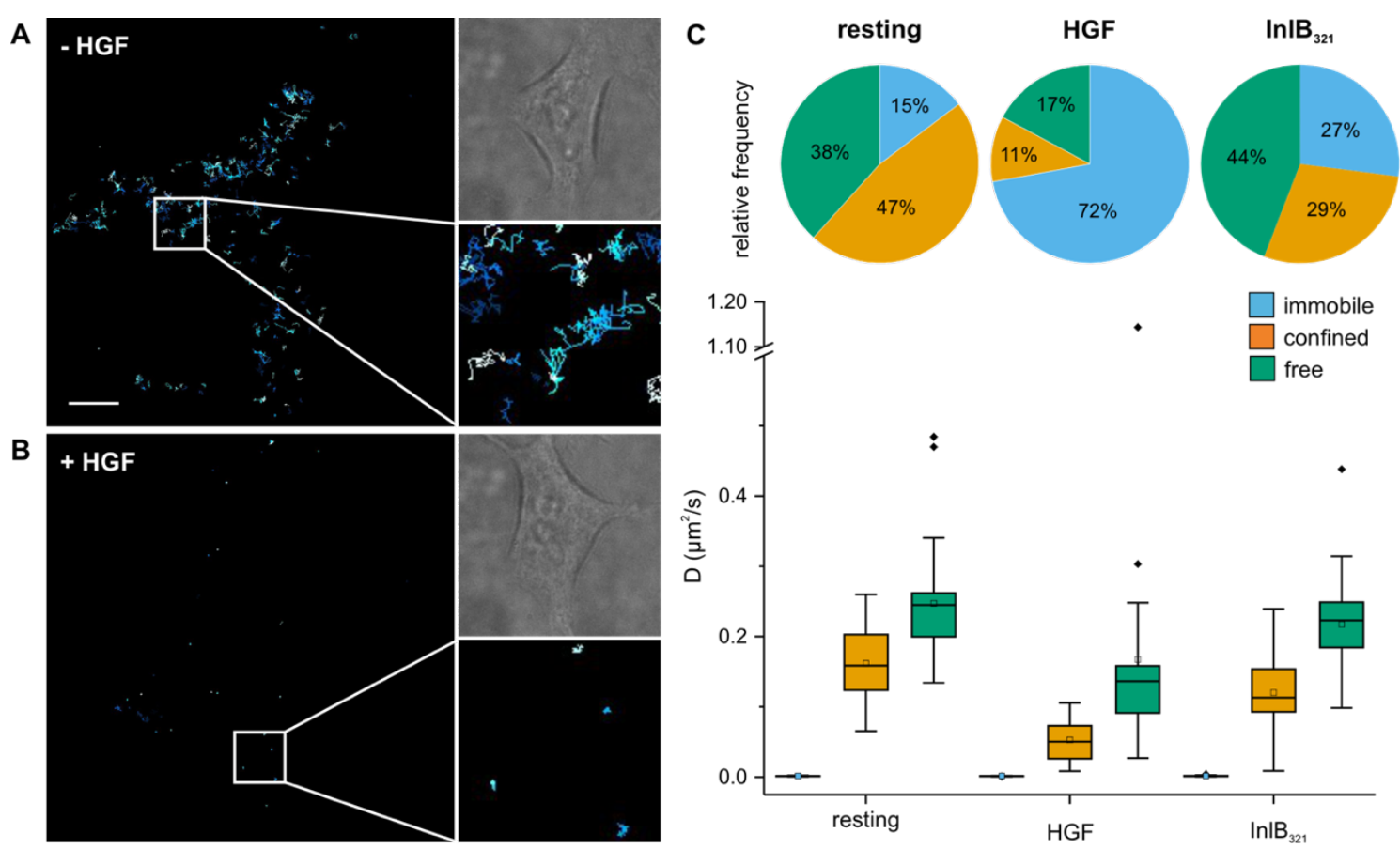

Figure 4.3.8: Single-particle-tracking of MET-mEos4b in resting and ligand-stimulated cells. (A, B) Trajectories of MET-mEos4b in resting (A) and HGF-stimulated (B) cells. Zoomins are shown in the bottom right. Brightfield images are shown in the top right. Scale bar is 5 $\mu \mathrm{m}$. (C) Pie charts of the relative frequency of the diffusion states for resting and ligandstimulated MET-mEos $4 \mathrm{~b}$ cells. Immobile as well as confined and freely diffusing receptors are depicted in the respective color. The mean diffusion coefficients of the individual diffusion states for resting and ligand-stimulated cells are represented in the box plot. The error is given as the standard error of the mean (-HGF, 32 cells; +HGF 33 cells; + InlB $_{321} 36$ cells). Adapted from Baldering et al (2020). Copyright CC BY 4.0.

Next, uPAINT (Giannone et al. 2010) measurements were performed using InlB $321^{-}$ ATTO $647 \mathrm{~N}$ which allows tracking of ligand-bound receptors. The MSD analysis was performed by Marie-Lena Harwardt. The anaylsis revealed the different diffusion states and their diffusion coefficients (Figure 4.3.9). The diffusion properties of the ligandbound MET receptor were compared with those of MET-mEos4b (sptPALM) in different cells, but both treated with the bacterial ligand. The immobile fraction was increased substantially from $27 \pm 3 \%$ to $44 \pm 3 \%$ in the uPAINT measurements and the confined state was clearly reduced (sptPALM: $29 \pm 4 \%$; uPAINT: $9 \pm 2 \%$ ). Diffusion 
coefficients decreased from $0.22 \pm 0.03 \mu \mathrm{m}^{2} / \mathrm{s}$ (free) and $0.12 \pm 0.03 \mu \mathrm{m}^{2} / \mathrm{s}$ (confined) in sptPALM experiments to $0.12 \pm 0.02 \mu \mathrm{m}^{2} / \mathrm{s}$ (free) and $0.05 \pm 0.02 \mu \mathrm{m}^{2} / \mathrm{s}$ (confined) in uPAINT measurements.
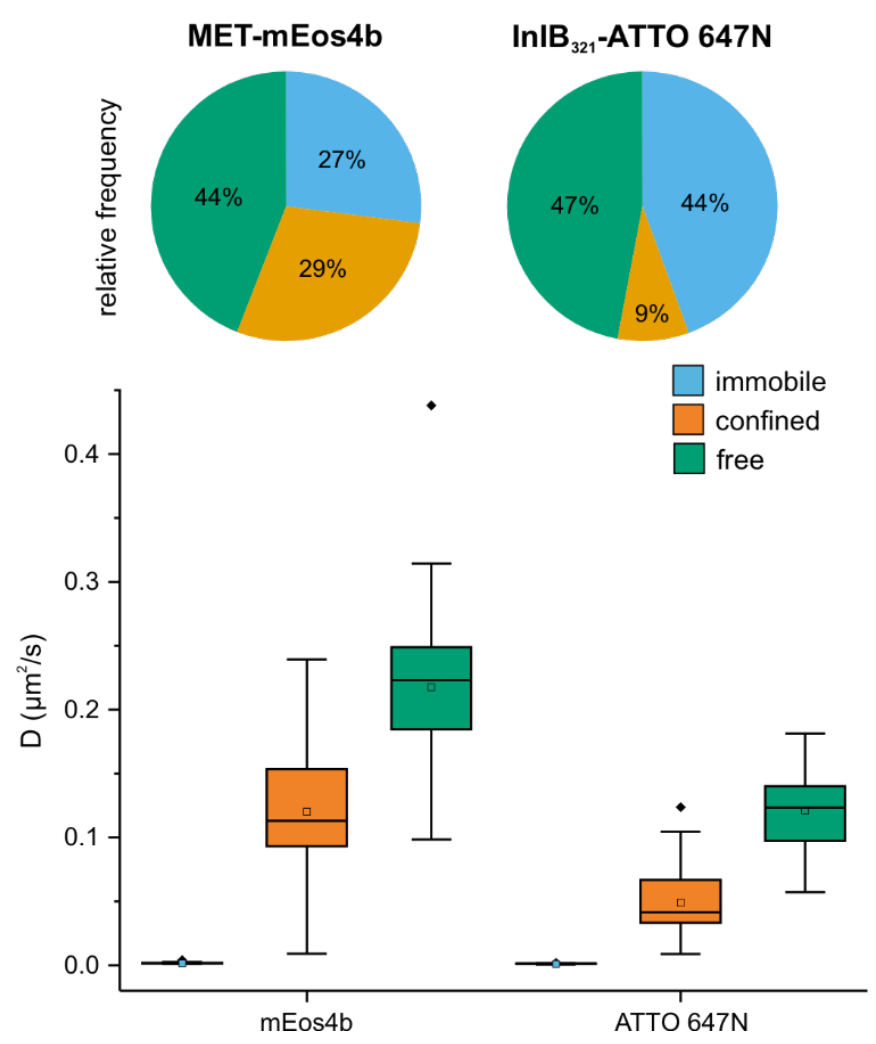

Figure 4.3.9: Comparison of the MSD analysis of sptPALM of MET-mEos4b and uPAINT

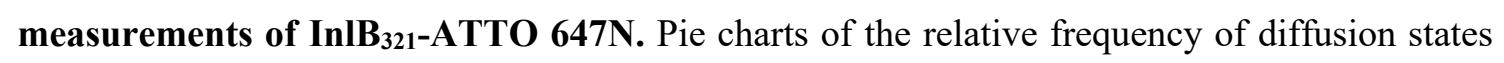
for sptPALM (mEos4b) and uPAINT experiments (ATTO 647N) of the MET C6 cell line. Immobile, confined, and free diffusing receptors are depicted in the respective color. The mean diffusion coefficients of the individual diffusion states for sptPALM and uPAINT experiments are represented as box plot. The error is given as the standard error of the mean ( 36 cells, mEos $4 \mathrm{~b}$; 34 cells, ATTO 647N). Adapted from Baldering et al (2020). Copyright CC BY 4.0.

Live-cell experiments of MET receptor in the presence and absence of ligands demonstrated a slowdown and immobilization of MET receptors after ligand treatment of HEK293T cells.

\subsubsection{Discussion}

Single-molecule localization microscopy techniques report on protein stoichiometry, spatial relations and molecular dynamics. To achieve this, stoichiometric and efficient labelling together with endogenous expression of the target protein in its native 
environment is necessary. This avoids externally added fluorophores, which may lead to high background signal or unspecific binding. Genetic engineering techniques such as CRISPR/Cas fulfill these criteria but especially achieving a high labeling efficiency in genetic engineering has not been trivial since many cells contain multiple sets of chromosomes that often are not targeted equally. Recent approaches of CRISPR/Cas9 in combination with PALM reported a labeling efficiency of tubulin-mEos3.2 below 20\%, estimated from the band intensities of western blots of wildtype and fusion proteins (Khan et al. 2017). Additionally, a codon-optimized version of mEos4b was reported to show an improved expression at the TubA1B locus (Khan et al. 2019) but still the labeling efficiency would not be ideal for qPALM experiments. However, there are also several examples in which high labeling efficiencies were achieved with genetic tagging by using CRISPR/Cas9. These high labeling efficiencies enabled multiple applications for analyzing proteins in their native environment. For example, Hansen et al. (2017) showed homozygous labeling of the CCCTC-binding factor (CTCF) with CRISPR/Cas9 in mouse embryonic stem (mES) cells, which allowed them to demonstrate that CTCF binds chromatin much more dynamically than cohesion (Hansen et al. 2017). In another study, labeling of RNA polyermase II with Dendra2 reported that RNA polymerase II clusters are short-lived in living cells (Cho et al. 2016).

\section{Generation of a stable MET-mEos4b HEK293T cell line by PCR tagging}

Here, CRISPR/Cas12a-assisted genome editing (PCR tagging) was used to label two receptor tyrosine kinases, the MET receptor and EGFR, endogenously with the fluorescent protein mEos4b. The EGFRmEos $4 \mathrm{~b}$ cell line showed only sparse labeling of endogenous receptors with mEos $4 \mathrm{~b}$. One reason for the low labeling efficiency of about $20 \%$ could be the labeling of only one chromosome, which might also be less frequently transcribed. Three copies of chromosome 7 (containing the EGFR and MET gene) were reported for HEK293T cells (Stepanenko et al. 2015). The analysis of more clones may provide a higher labeling efficiency suitable for further SMLM experiments. In contrast, PCR tagging has been shown to be very simple and time efficient in generating a stable MET-mEos4b HEK293T cell line enabling both stoichiometric and highly efficient labelling by maintaining an endogenous expression of the target protein. Here, two chromosomes containing the MET gene might be tagged with mEos4b and might be more frequently transcribed as the labeling efficiency is about $80 \%$. In addition, the high labeling efficiency, the functionality of the MET-mEos $4 b$ receptors could be 
demonstrated by western blots, showing the phosphorylation of receptors and signaling proteins after ligand stimulation with HGF and InlB $_{321}$. This allowed qPALM and sptPALM experiments on MET-mEos4b to study the oligomerization and membrane dynamics of the MET receptor (Figure 4.3.10).
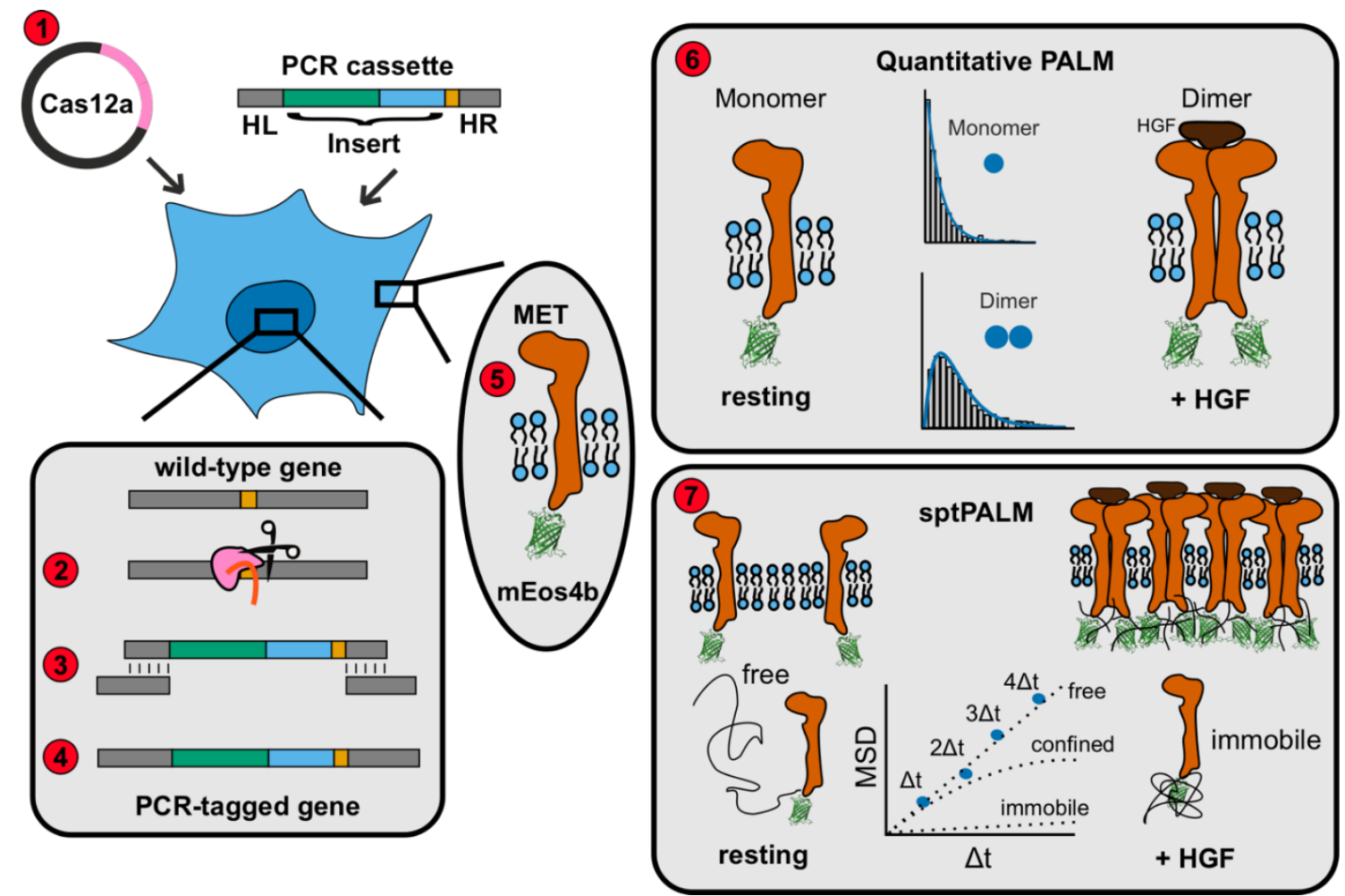

Figure 4.3.10: Possible applications of PCR tagging in combination with SMLM. (1) For

PCR tagging, Cas12a helper plasmid is co-transfected with the PCR cassette into the target cell line. (2) The Cas12a enzyme cuts the genomic DNA at the desired locus. (3) Homology directed repair leads to the insertion of the PCR cassette into the genome. (4) Schematic illustration of the PCR-tagged gene. (5) The target protein (here, MET, orange) is expressed with its tag (here, mEos4b; crystal structure of mEos2, PDB: 3S05; green). (6) The PCR-tagged cell line can be studied using quantitative PALM to distinguish monomers from dimers, exemplarily shown for the MET receptor (orange) with HGF (brown). (7) Single-particle tracking PALM is another application of PCR tagging and allows to characterize the dynamics of target proteins and distinguish different diffusion states. Adapted from Baldering et al (2020). Copyright CC BY 4.0.

Analysis of the oligomerization state of MET-mEos $4 b$ with addition of various ligands

Since the MET receptor was labeled almost stoichiometrically with mEos $4 \mathrm{~b}$, this allowed quantitative investigations. qPALM experiments of MET-mEos4b revealed a significant increase in dimerization upon stimulation with $1 \mathrm{nM}$ of its physiological ligand HGF (Figure 4.3.6). Furthermore, activation of MET-mEos4b with a higher concentration (5 
$\mathrm{nM}$ ) of the bacterial ligand $\mathrm{InlB}_{321}-\mathrm{ATTO} 647 \mathrm{~N}$ showed similar fractions of dimers (Figure 4.3.7). The addition of InlB $_{321}$-ATTO $647 \mathrm{~N}$ allowed not only to analyze all MET receptors, but also to select those receptors that colocalized with the InlB $_{321}$-ATTO $647 \mathrm{~N}$ fluorescence signal. The difference of the fraction of dimers between these conditions was small indicating that $\operatorname{InlB}_{321}$-ATTO $647 \mathrm{~N}$ was added at an almost saturating concentration, which is in the same range of a previously published $\mathrm{K}_{\mathrm{d}}$-value $(5 \mathrm{nM})$ (Dietz et al. 2013). Furthermore, HGF has a stronger binding affinity in the picomolar range (20-40 pM) (Higuchi und Nakamura 1991; Lokker et al. 1992), which is consistent with the lower concentration of HGF (1nM) used to observe an equal amount of MET dimers as for InlB $_{321}$-ATTO $647 \mathrm{~N}$. In photobleaching experiments of InlB 321 -ATTO $647 \mathrm{~N}$, a lower fraction of dimers was observed in the ligand-bound state (Dietz et al. 2013), however, at lower concentrations of InlB 321 -ATTO $647 \mathrm{~N}$ and using a construct with a degree of labeling of 0.5 .

Recently, FRET studies also indicated a high fraction of MET dimers in response to HGF (Li et al. 2020). Li et al. (2020) found strong donor fluorescence of Cy3 without stimulation, which indicates that MET receptors exist mainly as monomers. A substantial increase of acceptor fluorescence indicated the presence of MET dimers in HGFstimulated cells, in accordance with the results of the qPALM experiments of METmEos4b. In contrast to Li et al. (2020), this work here presents relative proportions of oligomers and not only provides evidence of an interaction between individual MET receptors in HGF-stimulated cells. Another study reported the downregulation of MET receptors through antibody-mediated receptor clustering ( $\mathrm{Li}$ et al. 2019). Li et al. (2019) show clustering of MET but do not demonstrate relative fractions of oligomers. However, in accordance with these studies (Dietz et al. 2013; Li et al. 2019; Li et al. 2020), a liganddependent dimerization of MET is demonstrated. The ligand-dependent dimerization was also found for other receptor tyrosine kinases, e.g. EGFR (Lemmon und Schlessinger 2010; Freed et al. 2017), which indicates a general characteristic for receptor tyrosine kinases. In sum, PCR tagging in combination with qPALM is a powerful tool to determine protein numbers in nanoscale clusters of target proteins at endogenous expression levels.

\section{Analysis of the dynamics of MET-mEos4b with addition of various ligands}

Besides the ligand-dependent dimerization of receptor tyrosine kinases, receptor slowdown and immobilization are expected after ligand binding (Chung 2017; Clarke und Martin-Fernandez 2019). In this work, live-cell single-particle tracking experiments of 
MET-mEos4b were conducted and showed a substantial increase in the immobile fraction after ligand stimulation. The effect was more pronounced by adding $1 \mathrm{nM}$ HGF to the cells than for $1 \mathrm{nM} \mathrm{InlB}_{321}$ (Figure 4.3.8). This is in accordance with the higher affinity of HGF to MET compared to InlB 321 (Higuchi und Nakamura 1991; Lokker et al. 1992; Dietz et al. 2013). In addition to an increase in the immobile fraction, the diffusion coefficients of free and confined states were also reduced to varying degrees by ligand stimulation (Figure 4.3.8). Similar to the increase in the immobile fraction, the decrease in diffusion coefficients was more pronounced in HGF-stimulated cells. The decrease in diffusion rates was also shown for MET in HeLa cells and similar diffusion coefficients were obtained (Harwardt et al. 2017). This shows the consistence of MET dynamics in different cell lines, and the functionality of the MET-mEos $4 b$ cell line. Furthermore, these diffusion coefficients also match published diffusion coefficients of other receptor tyrosine kinases (Michalet und Berglund 2012).

PCR tagging of MET-mEos4b also allowed to compare receptor mobility with the dynamics of the labeled ligand InlB $_{321}$-ATTO $647 \mathrm{~N}$ bound to MET. The uPAINT experiments showed nearly the same diffusion coefficients as measured in HeLa cells (Harwardt et al. 2017), while the immobile fraction was increased in MET-mEos4b cells (Figure 4.3.9). This is expected, since an increased ligand concentration of $1 \mathrm{nM}$ instead of $0.25 \mathrm{nM}$ was used in the uPAINT experiments with MET C6 cells. Single-particle tracking PALM of PCR-tagged stable cell lines in combination with uPAINT is perfectly suited for two-color single-particle tracking, since the fusion protein and the fluorophore of the labeled ligand can be spectrally separated. This could be beneficial in future experiments to analyze the association times of receptors and ligands in living cells.

In summary, PCR tagging is a simple and time-efficient tool to generate stable cell lines that offers many advantages in various applications of SMLM (Figure 4.3.10). Hence, this combination will help (i) to determine the oligomerization state and dynamics of other membrane proteins, (ii) to understand their molecular mechanisms, and (iii) to unravel their native function in future studies. The combination of these methods will be of particular interest for intracellular proteins, as PCR tagging avoids the need of harsh reagents for cell permeabilization in order to introduce a fluorophore label that also prevents the analysis in living cells. 


\subsection{Unbiased, automated analysis of single-molecule localization microscopy data}

The automation of analysis processes is of particular importance in science, as it is unbiased and allows a larger amount of statistics to be collected in less time. In qPALM analysis, a relatively large number of protein clusters are required to obtain a good result in model approximation, which requires time if done manually. In addition, automation simplifies complicated workflows and makes them available to non-experts. An automatic qPALM analysis termed quantitative algorithm for fluorescent kinetics analysis (QAFKA) was established in cooperation with the group of Yoav Shechtman (Department of Biomedical Engineering, Technion - Israel Institute of Technology, Haifa, Israel).

\subsubsection{Automated detection of single-molecule emission events}

qPALM analysis reported in chapters 4.1 and 4.3 employed manual cluster selection. In cooperation with Alon Saguy and Yoav Shechtman, a solution was sought for unbiased and automated detection of protein clusters in SMLM data, and subsequent approximation of a kinetic model to determine the oligomerization state. Alon Saguy developed a software package termed QAFKA which allows an automated analysis of qPALM data. This software package contains an automatic emitter recognition algorithm, extracts the intensity-time traces of the individual fluorophores and determines the number of blinking events (Figure 4.4.1). The frequency analysis of the blinking events is then fed into a neural network, which determines the proportion of monomers and dimers on a cell-to-cell basis.

QAFKA was initially trained and validated on simulated data of monomers and dimers. QAFKA achieved a higher accuracy (mean error $=2.8 \%$ ) compared to manual analysis (mean error $=5.5 \%$ ) for a number of only 128 clusters. Furthermore, QAFKA achieved lower mean errors with 100,000 simulated clusters (QAFKA: mean error $=0.19$; manual analysis: mean error $=0.48$ ). Hence, QAFKA consistently outperformed manual analysis in terms of accuracy, and reached a reasonable accuracy for low cluster numbers.

Next, QAFKA was evaluated using the experimental qPALM data presented in chapters 4.1 and 4.3. First, a monomeric reference protein mEos 3.2 was analyzed and identified as exclusively monomers (Figure 4.4.2 A) (see figure 4.1.2 for comparison with manual analysis). Next, a mixture of about $20 \%$ monomers and $80 \%$ dimers of mEos3.2 (genetic dimer) was analyzed with QAFKA (see figure 4.1.7 for comparison with manual 
analysis). QAFKA analysis determined $66 \pm 6 \%$ dimers and $34 \pm 6 \%$ monomers by correcting for a detection efficiency of $78 \%$ (see chapter 4.1.2). This demonstrates the applicability of QAFKA in in vitro experiments.

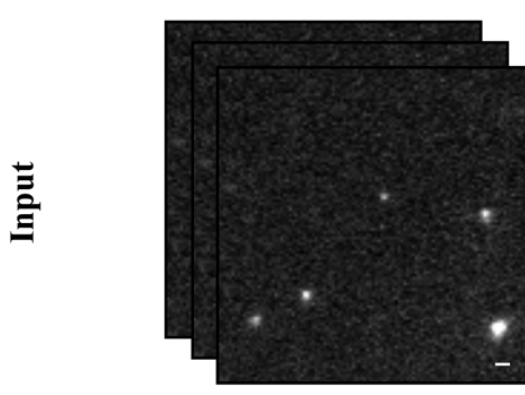

qPALM video

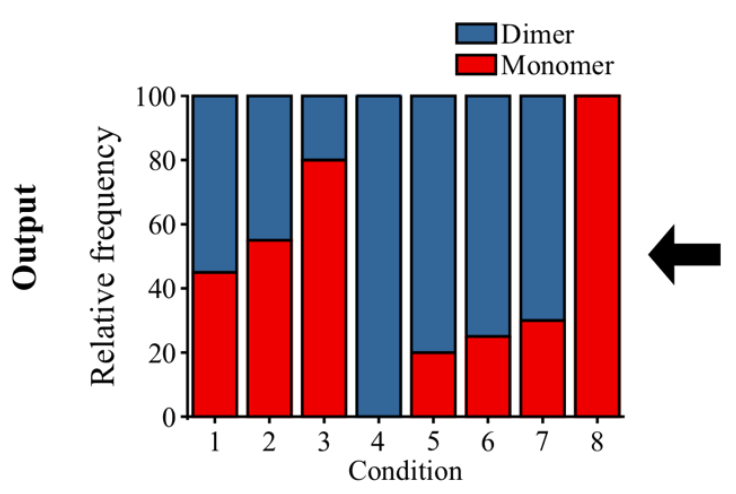

Fractions of oligomers

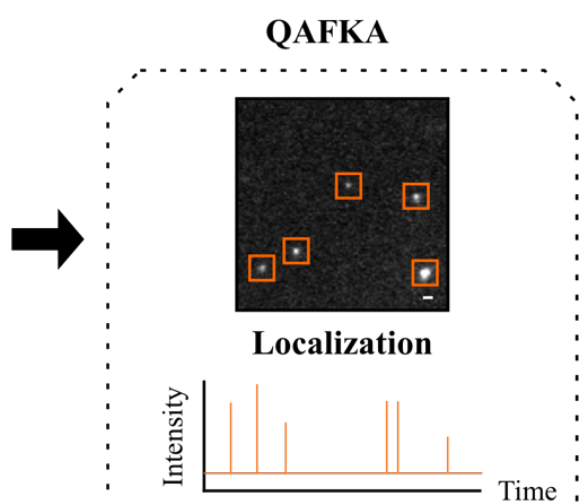

Feature extraction

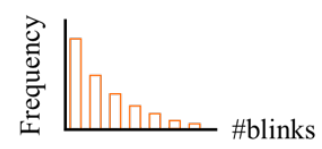

Blinking distribution

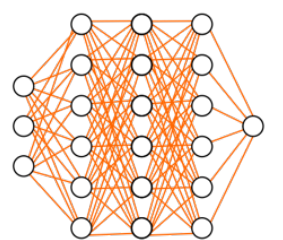

Deep neural network

Figure 4.4.1: Workflow of the QAFKA software. A qPALM video serves as input. QAFKA localizes single emitters and extracts features such as the number of blinking events. A neural network estimates the fractions of monomers and dimers according to the distribution of the number of blinking events. The scale bar is $1 \mu \mathrm{m}$.

\subsubsection{Automated, unbiased quantification of protein monomers and dimers in cells}

In order to validate QAFKA in situ, the monomeric and dimeric membrane proteins CD86 and CTLA-4 fused to mEos3.2 were analyzed. For monomeric CD86-mEos3.2, QAFKA analysis revealed mainly monomers $(98 \pm 4 \%)$. In contrast, exclusively dimers $(99 \pm$ 6\%) were determined in cells expressing CTLA-4-mEos3.2 (Figure 4.4.2 A, compare with figure 4.1.8).

Next, QAFKA was used to analyze HGF-stimulated and unstimulated MET-mEos4b receptors. An unstimulated MET-mEos4b cell showed 26\% dimers, whereas $64 \%$ dimers were observed in the HGF-stimulated MET-mEos4b cell (Figure 4.4.2 A). An increase in the fraction of dimers was also observed upon HGF addition in chapter 4.3 (Figure 4.3.6). 
QAFKA allowed the analysis of single cells and thus to explore the heterogeneity between cells. The relative frequency of dimers was determined for 24 unstimulated MET-mEos $4 \mathrm{~b}$ cells (measured by Christos Karathanasis) and plotted against their cluster density (Figure 4.4.2 B). The number of dimers clearly correlates with the cluster density. This demonstrates that QAFKA facilitates a single-cell analysis by analyzing all MET$m E o s 4 b$ clusters at the cellular membrane in a time-efficient way.

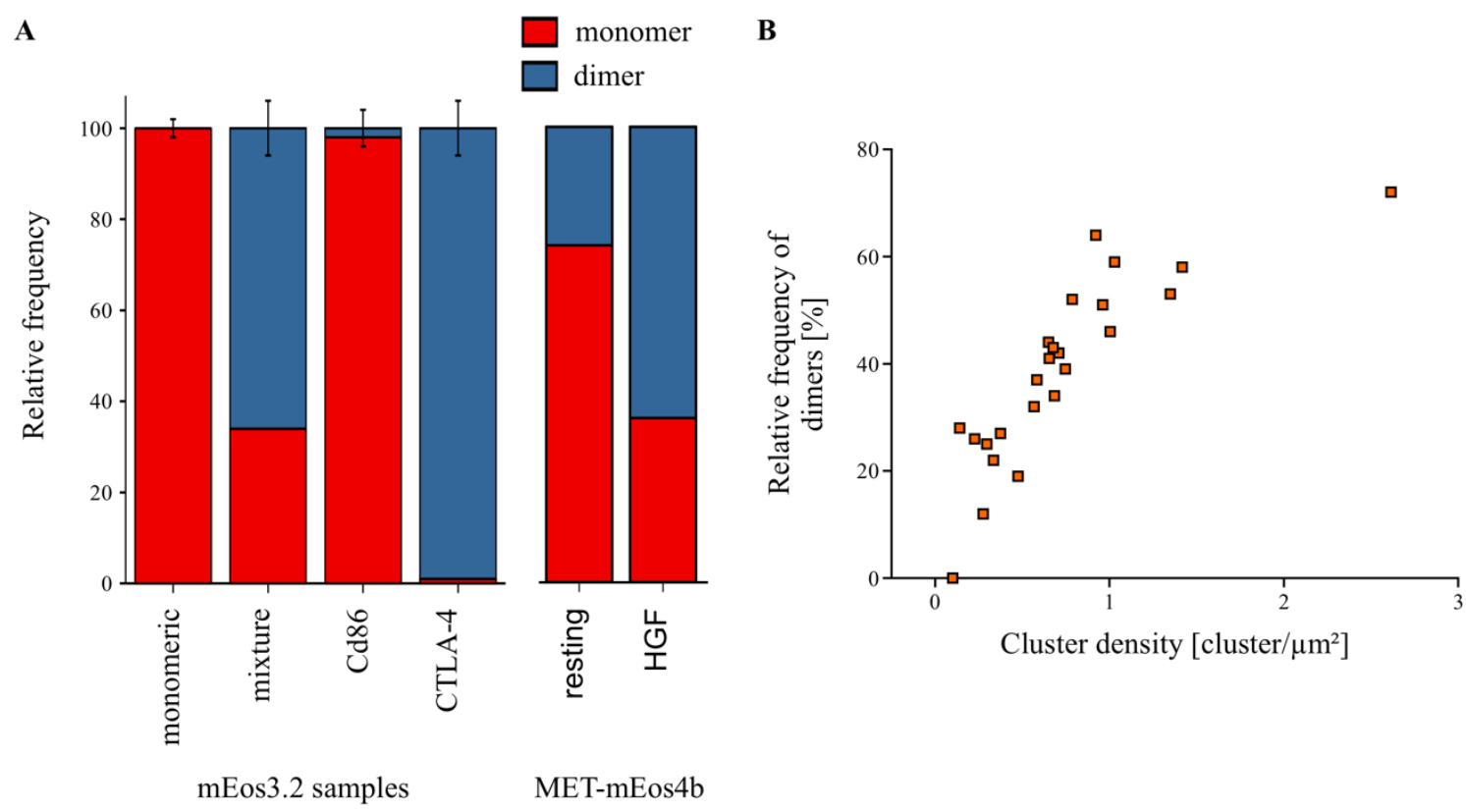

Figure 4.4.2: QAFKA determines protein monomers and dimers in cells. (A) QAFKA predicts relative frequency of monomers and dimers of (i) single mEos3.2 molecules and (ii) a mixture of monomers and dimers (20:80) in vitro as well as (iii) monomeric CD86-mEos3.2, and (iv) dimeric CTLA-4-mEos3.2 in HEK293T cells (left). Relative fractions of monomers and dimers of an unstimulated and a HGF-stimulated MET-mEos $4 \mathrm{~b}$ cell predicted by QAFKA (right). (B) Relative frequency of dimers plotted against the cluster density in unstimulated MET-mEos $4 \mathrm{~b}$ cells. Each point represents a single cell.

\subsubsection{Discussion}

The quantitative analysis pipeline of SMLM data can be complex, time-consuming and underlies human bias. With QAFKA, a software tool for automated and unbiased analysis became available. The analysis automation of qPALM data with QAFKA showed excellent performance with simulated data (128-100,000 cluster). A major advantage of QAFKA is the speed of the analysis, which is at least 60 times faster than manual analysis. Hence, the automation with QAFKA allows a high number of statistics to be collected in 
a reasonable amount of time. In addition, QAFKA showed a slightly higher accuracy in the analysis of blinking histograms.

The quantitative results of QAFKA on experimental PALM data are comparable to previous manual analysis. Single mEos3.2 molecules deposited on a glass surface were assigned as monomers as expected (Figure 4.4.2 A). The mixture of monomers and dimers of $m E 0$ B 3.2 contained about $80 \%$ dimers from which $66 \%$ were assigned as dimers by QAFKA. The difference may result from background that is localized with QAFKA and increases the monomer fraction. The manual analysis showed $82 \%$ dimers and thus lead to more precise results (section 4.1.2). However, the results of manual analysis might have a human bias, which is excluded by an automated analysis workflow.

The next and important step was to establish QAFKA to analyze protein monomers and dimers in the plasma membrane of cells. QAFKA analysis correctly reported that CD86mEos3.2 is monomeric and that CTLA-4-mEos3.2 is dimeric. Reference proteins were thus correctly assigned in their oligomeric state, which demonstrates the applicability of QAFKA for quantification.

Next, QAFKA was applied on the qPALM data of MET-mEos4b in HEK293T cells. In an unstimulated cell, QAFKA determined less MET dimers than in a HGF-stimulated cell. This result matches previous findings (see figure 4.3.6) and confirms the ligandinduced dimerization of receptor tyrosine kinases (Schlessinger 2002). Finally, singlecell analysis of unstimulated MET-mEos $4 \mathrm{~b}$ cells showed a dependence of the number of dimers on the MET receptor density on the plasma membrane. Such a strong increase in dimers is not expected and indicates that QAFKA might combine neighboring clusters to one cluster. However, this effect needs to be evaluated in more detail in future experiments, as single-cell analysis would have numerous applications, such as detecting the heterogeneity of dimerization between cells or abnormal expression levels and dimer ratios of membrane proteins associated with common diseases.

In summary, QAFKA showed a reliable quantification of reference structures, confirmed an increase in MET dimers upon ligand stimulation and allowed single-cell analysis. Main advantages are the analysis speed and accuracy as well as the exclusion of userdependent bias. A possible drawback is the inclusion of background signal, which may increase the monomer fraction. 


\section{Conclusion \& Outlook}

Understanding the organization of proteins in molecular complexes reports on their function in health and disease. Single-molecule localization microscopy provides the spatial resolution that allows the visualization of such protein complexes in the small nanometer range (Heilemann et al. 2009; Sauer und Heilemann 2017; Strauss et al. 2018). However, individual proteins in molecular assemblies are still too small to be resolved. In the last decade, quantitative approaches have been developed that offer alternative methods for determining protein numbers (Sengupta et al. 2011; Lee et al. 2012; Puchner et al. 2013; Hummer et al. 2016; Nasu et al. 2016; Jungmann et al. 2016; Zanacchi et al. 2017). These methods are not exclusively based on spatial resolution but gain information from other experimental observables, such as the kinetics of fluorophore blinking or label binding.

Quantitative PALM has proven to be well suited for the analysis of small oligomers labeled with photoconvertible fluorescent proteins (Fricke et al. 2015; Hummer et al. 2016). In this thesis, the two critical parameters in qPALM, the bleaching probability and the detection efficiency, were investigated both practically (section 4.1) and theoretically (section 4.2). Based on these findings, the oligomeric state of the MET receptor in the presence and absence of ligands was analyzed by creating a CRISPR/Cas12a-engineered stable cell line expressing MET-mEos4b (section 4.3). Furthermore, an automated analysis tool enabled qPALM analysis within minutes instead of hours, with results similar or better to those obtained by manual analysis (section 4.4).

\subsection{Calibration references for quantitative PALM}

Different fluorescent proteins commonly used in PALM were analyzed in vitro regarding their blinking kinetics. The fluorescent proteins mEos3.2, mEos $4 \mathrm{~b}$, and mMaple3 were found to be suitable for quantitative PALM experiments because a clear distinction between signal and background is possible. In addition, the importance of amino acid 69 for blinking kinetics was demonstrated by mutating this particular residue in mEos3.2 and Dendra2. Furthermore, it was shown that different fluorescent proteins (mEos3.2, mMaple3) exhibit different blinking kinetics when reducing agent was added. Also, in this case the amino acid 69 may play a central role. In summary, these experiments 
propose a major role of amino acid 69 for the blinking properties of fluorescent proteins. Further mutations would help to characterize how blinking properties are influenced by the amino acids involved in fluorophore blinking and which type of amino acid leads to high number of blinking events. This would allow to create an improved fluorescent protein for quantification and together with structural studies indicate the arrangement of the dark states.

The fluorescent protein mEos3.2 was used to generate synthetic and genetic dimers and confirmed that qPALM can report the fraction of undetected molecules and in general differentiate monomers from dimers. Reference proteins of mEos 3.2 and mEos $4 \mathrm{~b}$ revealed similar $p$ - and $q$-values in situ as determined for mEos2 (Fricke et al. 2015; Hummer et al. 2016; Krüger et al. 2017; Karathanasis et al. 2020). Next, it would be interesting to investigate whether qPALM can also be used for the analysis of higherorder oligomers. qPALM experiments have already shown that the TNF receptor 1 exists in the ligand-bound state in trimers and higher-ordered oligomers (Karathanasis et al. 2020). However, it is still unclear up to which oligomer size qPALM experiments provide reliable information for quantification in practice. Therefore, the analysis of reference proteins of higher-ordered oligomers is a key step to identify the limits of qPALM. In addition, quantification in live cells would be highly interesting, because the dynamic association and dissociation would give rise to the lifetimes of such protein complexes.

\subsection{Extending the theory of qPALM}

In order to obtain reliable quantitative results of higher-order oligomers, a kinetic model is required that adequately describes the respective processes. In addition to extending the toolbox of fluorescent proteins suitable for qPALM and their precise characterization, this work provides the necessary theoretical background for the exact determination of fractions of different oligomers by including a detection probability instead of a $q$-value (Hummer et al. 2016; Baldering et al. 2019a). In simulations, higher-order oligomers were precisely predicted by the revised model, including oligomers that are not detected at all, e.g. due to incomplete chromophore maturation. It was shown that the deviations with respect to the existing literature (Hummer et al. 2016; Krüger et al. 2017; Karathanasis et al. 2020) are almost negligible, since mainly small oligomers were analyzed. The use of the revised model is recommended for future qPALM experiments. In the future, experiments on higher-order oligomers have to confirm whether the model is well applicable in practice. A comparison of the performance of qPALM and qPAINT 
with regard to the quantitative analysis of higher-order oligomers would be interesting. qPAINT offers the advantage that every single protein cluster might be assigned to the respective oligomeric state, whereas qPALM only allows the ensemble oligomeric state to be determined. However, qPALM benefits from stoichiometric labeling, which is more difficult in qPAINT. In summary, it was proven that the revised model describes higherorder oligomers more precisely and also includes oligomers that are not detected at all.

\subsection{CRISPR/Cas12a-assisted endogenous protein labeling for super- resolution microscopy}

This thesis demonstrates CRISPR/Cas12a-assisted endogenous protein labeling of receptor tyrosine kinases for super-resolution microscopy. EGFR and the MET receptor were endogenously tagged with mEos4b in HEK293T cells. The labeling efficiency of EGFR-mEos4b was low ( 20\%), while MET-mEos4b cells showed a high labeling efficiency of $\sim 81 \%$. This enabled the analysis of MET receptor organization in protein assemblies and demonstrated a ligand-induced dimerization after ligand binding, which is consistent with other results in the literature (Schlessinger 2002). In several studies also higher-order oligomers such as tetramers were observed for EGFR (Clayton et al. 2005; Huang et al. 2016; Needham et al. 2016). However, in order to be able to quantify EGFR, a higher labeling efficiency would be advantageous. A promising approach would be to use the codon-optimized sequence of mEos $4 b$ published in Khan et al. (2019). This may lead to an improved translation of the fluorescent protein and might increase the labeling efficiency. Another attempt would be to use e.g. nocodazole or other small molecules to synchronize the cell cycle, which has previously been shown to increase the tagging efficiency (Lin et al. 2014). Besides qPALM experiments, sptPALM showed that MET receptors are slowed by the addition of their ligands, HGF and InlB $321-A T T O 647 \mathrm{~N}$. This is also consistent with previous literature (Harwardt et al. 2017) and hints at a ligandinduced immobilization of MET. A combination of two tracking methods, sptPALM and uPAINT, would be perfectly suited for two-color single-particle tracking, since the fluorescence of the fluorescent protein and the fluorophore of the labeled ligand may be spectrally separated. This would be beneficial to analyze the dissociation constants of ligands and receptors in living cells. In summary, this work has proven that CRISPR/Cas12a-assisted PCR tagging allows efficient endogenous labeling of membrane proteins, which can subsequently be analyzed with qPALM and sptPALM. 
In addition to receptor tyrosine kinases, other types of membrane proteins could be labeled by CRISPR/Cas12a. For example, the arrangement of pore complexes, transporters or ion channels could be assessed with quantitative PALM. Furthermore, the analysis of the dynamics of these membrane proteins would be interesting to learn more about different activation mechanisms that may help to treat common diseases associated with these proteins.

Another approach with a high impact would be to combine peptide-PAINT (Eklund et al. 2020) with CRISPR/Cas12a. Small peptides would be used as endogenous tags and would allow a quantitative analysis by qPAINT. Since stoichiometric labeling can be guaranteed by CRISPR/Cas12a, qPAINT analysis would provide access to analysis on the composition of higher-order oligomers.

Research on the signal transduction of receptor tyrosine kinases now focuses on the arrangement of and interaction with signaling proteins. These proteins are often multivalent and thus allow diverse interactions that lead to a network of proteins. According to that, phase separation models of signaling proteins have been presented (Huang et al. 2016; Mayer und Yu 2018; Huang et al. 2019). However, the exact formation and the leading forces are unclear. Live-cell experiments with near-molecular resolution on signaling proteins can track the formation of multiprotein assemblies and the role of phase separation in receptor signaling.

\subsection{Quantification with QAFKA surpasses manual analysis}

Recent advances in computational science represent an impressive ability to accelerate complex analysis pipelines and provide computational predictions that enable researchers to decipher previously impossible problems. Especially in biological imaging with e.g. super-resolution techniques, deep learning algorithms show fascinating improvements and results (Strack 2018; Moen et al. 2019; Belthangady und Royer 2019).

The automated analysis of qPALM data with QAFKA was successfully established and validated on simulated data and outperformed manual analysis both in accuracy and analysis speed. QAFKA demonstrated reliable quantification of various reference proteins in in vitro and in situ experiments making QAFKA a valuable tool for future quantification. The application of QAFKA on MET-mEos4b cells showed an increase in dimer formation upon ligand stimulation as demonstrated in chapter 4.3. The fast and comprehensive analysis with QAFKA allowed single-cell analysis. Single-cell analysis is of great importance because it allows to determine the heterogeneity of dimerization 
rates in cells as well as abnormal expression levels and dimer ratios of membrane proteins associated with common diseases. However, the strong increase in dimerization of unstimulated MET-mEos $4 \mathrm{~b}$ cells according to a small increase in its cluster density might indicate a bias in the QAFKA analysis pipeline. Here, varying analysis parameters might give insight into the effect seen with QAFKA. In the future, the establishment of QAFKA in the determination of monomer and dimer ratios in living cells would be very interesting. This would allow the dynamics of assembly and disassembly to be monitored. In addition, QAFKA could assign individual protein clusters to a respective oligomeric state or differentiate various fluorophores or labels. This could be facilitated by combining QAFKA with DNA-PAINT or quantitative PAINT (Jungmann et al. 2016), as QAFKA could learn the emission pattern of a defined number of docking sites as well as the emission pattern of different fluorophores or lengths of imager strands. These experiments would allow quantitative multicolor imaging and provide oligomeric states of different proteins in the same protein complex. 


\section{References}

Abbe, E. (1873): Beiträge zur Theorie des Mikroskops und der mikroskopischen Wahrnehmung. In: Archiv f. mikrosk. Anatomie 9 (1), S. 413-468. DOI: 10.1007/BF02956173.

Airy, G. B. (1835): On the diffraction of an object-glass with circular aperture. In: Transactions of the Cambridge Philosophical Society 1835 (5), S. 283.

Ambrose, E. J. (1956): A surface contact microscope for the study of cell movements. In: Nature 178 (4543), S. 1194. DOI: 10.1038/1781194a0.

Ando, Ryoko; Mizuno, Hideaki; Miyawaki, Atsushi (2004): Regulated fast nucleocytoplasmic shuttling observed by reversible protein highlighting. In: Science (New York, N.Y.) 306 (5700), S. 1370-1373. DOI: 10.1126/science.1102506.

Annibale, P.; Scarselli, M.; Kodiyan, A.; Radenovic, A. (2010): Photoactivatable Fluorescent Protein mEos2 Displays Repeated Photoactivation after a Long-Lived Dark State in the Red Photoconverted Form. In: J. Phys. Chem. Lett. 1 (9), S. 1506-1510. DOI: $10.1021 / \mathrm{jz} 1003523$.

Annibale, Paolo; Vanni, Stefano; Scarselli, Marco; Rothlisberger, Ursula; Radenovic, Aleksandra (2011): Quantitative photo activated localization microscopy. Unraveling the effects of photoblinking. In: PloS one 6 (7), e22678. DOI:

10.1371/journal.pone.0022678.

Aoki, Valéria; Sousa, Joaquim X.; Fukumori, Lígia M. I.; Périgo, Alexandre M.; Freitas, Elder L.; Oliveira, Zilda N. P. (2010): Direct and indirect immunofluorescence. In: Anais brasileiros de dermatologia 85 (4), S. 490-500. DOI: 10.1590/s036505962010000400010.

Axelrod, D. (2001): Total internal reflection fluorescence microscopy in cell biology. In: Traffic (Copenhagen, Denmark) 2 (11), S. 764-774. DOI: 10.1034/j.16000854.2001.21104.x.

Axelrod, D.; Burghardt, T. P.; Thompson, N. L. (1984): Total internal reflection fluorescence. In: Annual review of biophysics and bioengineering 13, S. 247-268. DOI: 10.1146/annurev.bb.13.060184.001335.

Baker, Susan M.; Buckheit, Robert W.; Falk, Matthias M. (2010): Green-to-red photoconvertible fluorescent proteins: tracking cell and protein dynamics on standard wide-field mercury arc-based microscopes. In: BMC cell biology 11, S. 15. DOI: 10.1186/1471-2121-11-15.

Baldanzi, Gianluca; Graziani, Andrea (2014): Physiological Signaling and Structure of the HGF Receptor MET. In: Biomedicines 3 (1), S. 1-31. DOI: 10.3390/biomedicines3010001.

Baldering, Tim N.; Bullerjahn, Jakob T.; Hummer, Gerhard; Heilemann, Mike; Malkusch, Sebastian (2019a): Molecule counts in complex oligomers with singlemolecule localization microscopy. In: J. Phys. D: Appl. Phys. 52 (47), S. 474002. DOI: 10.1088/1361-6463/ab3b65. 
Baldering, Tim N.; Dietz, Marina S.; Gatterdam, Karl; Karathanasis, Christos; Wieneke, Ralph; Tampé, Robert; Heilemann, Mike (2019b): Synthetic and genetic dimers as quantification ruler for single-molecule counting with PALM. In: Molecular biology of the cell 30 (12), S. 1369-1376. DOI: 10.1091/mbc.E18-10-0661.

Baldering, Tim N.; Karathanasis, Christos; Harwardt, Marie-Lena I.E.; Freund, Petra; Meurer, Matthias; Rahm, Johanna V. et al. (2020): CRISPR/Cas12a-mediated labeling of MET receptor enables quantitative single-molecule imaging of endogenous protein organization and dynamics. In: iScience, S. 101895. DOI: 10.1016/j.isci.2020.101895.

Balzarotti, Francisco; Eilers, Yvan; Gwosch, Klaus C.; Gynnå, Arvid H.; Westphal, Volker; Stefani, Fernando D. et al. (2017): Nanometer resolution imaging and tracking of fluorescent molecules with minimal photon fluxes. In: Science (New York, N.Y.) 355 (6325), S. 606-612. DOI: 10.1126/science.aak9913.

Banan, Mehdi (2020): Recent advances in CRISPR/Cas9-mediated knock-ins in mammalian cells. In: Journal of biotechnology 308, S. 1-9. DOI:

10.1016/j.jbiotec.2019.11.010.

Barnard, T. J.; Yu, X.; Noinaj, N.; Taraska, J. W. (2014): Crystal Structure of Green Fluorescent Protein.

Barrangou, Rodolphe; Fremaux, Christophe; Deveau, Hélène; Richards, Melissa; Boyaval, Patrick; Moineau, Sylvain et al. (2007): CRISPR provides acquired resistance against viruses in prokaryotes. In: Science (New York, N.Y.) 315 (5819), S. 1709-1712. DOI: $10.1126 /$ science.1138140.

Beer (1852): Bestimmung der Absorption des rothen Lichts in farbigen Flüssigkeiten. In: Ann. Phys. 162 (5), S. 78-88. DOI: 10.1002/andp.18521620505.

Belthangady, Chinmay; Royer, Loic A. (2019): Applications, promises, and pitfalls of deep learning for fluorescence image reconstruction. In: Nature methods 16 (12), S. 1215-1225. DOI: 10.1038/s41592-019-0458-z.

Bergendahl, L. Therese; Marsh, Joseph A. (2017): Functional determinants of protein assembly into homomeric complexes. In: Scientific reports 7 (1), S. 4932 . DOI: 10.1038/s41598-017-05084-8.

Bertero, Alessandro; Brown, Stephanie; Vallier, Ludovic (2017): Methods of Cloning. In: Basic Science Methods for Clinical Researchers: Elsevier, S. 19-39.

Betzig, Eric; Patterson, George H.; Sougrat, Rachid; Lindwasser, O. Wolf; Olenych, Scott; Bonifacino, Juan S. et al. (2006): Imaging intracellular fluorescent proteins at nanometer resolution. In: Science (New York, N.Y.) 313 (5793), S. 1642-1645. DOI: 10.1126/science. 1127344 .

Bhatia, Sumeena; Edidin, Michael; Almo, Steven C.; Nathenson, Stanley G. (2005): Different cell surface oligomeric states of B7-1 and B7-2. Implications for signaling. In: Proceedings of the National Academy of Sciences of the United States of America 102 (43), S. 15569-15574. DOI: 10.1073/pnas.0507257102.

Birchmeier, Carmen; Birchmeier, Walter; Gherardi, Ermanno; Vande Woude, George F. (2003): Met, metastasis, motility and more. In: Nature reviews. Molecular cell biology 4 (12), S. 915-925. DOI: 10.1038/nrm1261.

Bladt, F.; Riethmacher, D.; Isenmann, S.; Aguzzi, A.; Birchmeier, C. (1995): Essential role for the c-met receptor in the migration of myogenic precursor cells into the limb bud. In: Nature 376 (6543), S. 768-771. DOI: 10.1038/376768a0. 
Blom, Hans; Widengren, Jerker (2017): Stimulated Emission Depletion Microscopy. In: Chemical reviews 117 (11), S. 7377-7427. DOI: 10.1021/acs.chemrev.6b00653.

Blume-Jensen, P.; Hunter, T. (2001): Oncogenic kinase signalling. In: Nature 411 (6835), S. 355-365. DOI: 10.1038/35077225.

Böger, Carolin; Hafner, Anne-Sophie; Schlichthärle, Thomas; Strauss, Maximilian T.; Malkusch, Sebastian; Endesfelder, Ulrike et al. (2019): Super-resolution imaging and estimation of protein copy numbers at single synapses with DNA-point accumulation for imaging in nanoscale topography. In: Neurophotonics 6 (3), S. 35008. DOI: 10.1117/1.NPh.6.3.035008.

Bolotin, Alexander; Quinquis, Benoit; Sorokin, Alexei; Ehrlich, S. Dusko (2005): Clustered regularly interspaced short palindrome repeats (CRISPRs) have spacers of extrachromosomal origin. In: Microbiology (Reading, England) 151 (Pt 8), S. 25512561. DOI: 10.1099/mic.0.28048-0.

Bourgeois, Dominique (2017): Deciphering Structural Photophysics of Fluorescent Proteins by Kinetic Crystallography. In: International journal of molecular sciences 18 (6). DOI: 10.3390/ijms18061187.

Braun, L.; Ohayon, H.; Cossart, P. (1998): The InIB protein of Listeria monocytogenes is sufficient to promote entry into mammalian cells. In: Molecular microbiology 27 (5), S. 1077-1087. DOI: 10.1046/j.1365-2958.1998.00750.x.

Brouns, Stan J. J.; Jore, Matthijs M.; Lundgren, Magnus; Westra, Edze R.; Slijkhuis, Rik J. H.; Snijders, Ambrosius P. L. et al. (2008): Small CRISPR RNAs guide antiviral defense in prokaryotes. In: Science (New York, N.Y.) 321 (5891), S. 960-964. DOI: 10.1126/science.1159689.

Cho, Won-Ki; Jayanth, Namrata; Mullen, Susan; Tan, Tzer Han; Jung, Yoon J.; Cissé, Ibrahim I. (2016): Super-resolution imaging of fluorescently labeled, endogenous RNA Polymerase II in living cells with CRISPR/Cas9-mediated gene editing. In: Scientific reports 6, S. 35949. DOI: 10.1038/srep35949.

Chudakov, Dmitriy M.; Matz, Mikhail V.; Lukyanov, Sergey; Lukyanov, Konstantin A. (2010): Fluorescent proteins and their applications in imaging living cells and tissues. In: Physiological reviews 90 (3), S. 1103-1163. DOI: 10.1152/physrev.00038.2009.

Chung, Inhee (2017): Optical measurement of receptor tyrosine kinase oligomerization on live cells. In: Biochimica et biophysica acta. Biomembranes 1859 (9 Pt A), S. 14361444. DOI: 10.1016/j.bbamem.2017.03.026.

Citri, Ami; Yarden, Yosef (2006): EGF-ERBB signalling: towards the systems level. In: Nature reviews. Molecular cell biology 7 (7), S. 505-516. DOI: 10.1038/nrm1962.

Clarke, David T.; Martin-Fernandez, Marisa L. (2019): A Brief History of SingleParticle Tracking of the Epidermal Growth Factor Receptor. In: Methods and protocols 2 (1). DOI: $10.3390 / \mathrm{mps} 2010012$.

Clayton, Andrew H. A.; Walker, Francesca; Orchard, Suzanne G.; Henderson, Christine; Fuchs, Dominik; Rothacker, Julie et al. (2005): Ligand-induced dimertetramer transition during the activation of the cell surface epidermal growth factor receptor-A multidimensional microscopy analysis. In: The Journal of biological chemistry 280 (34), S. 30392-30399. DOI: 10.1074/jbc.M504770200.

Collins, Alison V.; Brodie, Douglas W.; Gilbert, Robert J.C.; Iaboni, Andrea; MansoSancho, Raquel; Walse, Björn et al. (2002): The Interaction Properties of Costimulatory 
Molecules Revisited. In: Immunity 17 (2), S. 201-210. DOI: 10.1016/s10747613(02)00362-X.

Combs, Christian A. (2010): Fluorescence microscopy: a concise guide to current imaging methods. In: Current protocols in neuroscience Chapter 2, Unit2.1. DOI: 10.1002/0471142301.ns0201s50.

Comoglio, Paolo M.; Trusolino, Livio; Boccaccio, Carla (2018): Known and novel roles of the MET oncogene in cancer. A coherent approach to targeted therapy. In: Nature reviews. Cancer 18 (6), S. 341-358. DOI: 10.1038/s41568-018-0002-y.

Condon, Edward U. (1928): Nuclear Motions Associated with Electron Transitions in Diatomic Molecules. In: Phys. Rev. 32 (6), S. 858-872. DOI: 10.1103/PhysRev.32.858.

Coons, A. H.; Creech, H. J.; Jones, R. N. (1941): Immunological Properties of an Antibody Containing a Fluorescent Group. In: Experimental Biology and Medicine 47 (2), S. 200-202. DOI: 10.3181/00379727-47-13084P.

Coons, A. H.; KAPLAN, M. H. (1950): Localization of antigen in tissue cells; improvements in a method for the detection of antigen by means of fluorescent antibody. In: The Journal of experimental medicine 91 (1), S. 1-13. DOI:

10.1084/jem.91.1.1.

Cooper, C. S.; Park, M.; Blair, D. G.; Tainsky, M. A.; Huebner, K.; Croce, C. M.; Vande Woude, G. F. (1984): Molecular cloning of a new transforming gene from a chemically transformed human cell line. In: Nature 311 (5981), S. 29-33. DOI: $10.1038 / 311029 \mathrm{a} 0$.

Critchley, William R.; Pellet-Many, Caroline; Ringham-Terry, Benjamin; Harrison, Michael A.; Zachary, Ian C.; Ponnambalam, Sreenivasan (2018): Receptor Tyrosine Kinase Ubiquitination and De-Ubiquitination in Signal Transduction and Receptor Trafficking. In: Cells 7 (3). DOI: 10.3390/cells7030022.

Darlington, Peter J.; Kirchhof, Mark G.; Criado, Gabriel; Sondhi, Jitin; Madrenas, Joaquín (2005): Hierarchical regulation of CTLA-4 dimer-based lattice formation and its biological relevance for T cell inactivation. In: Journal of immunology (Baltimore, Md. : 1950) 175 (2), S. 996-1004. DOI: 10.4049/jimmunol.175.2.996.

Deltcheva, Elitza; Chylinski, Krzysztof; Sharma, Cynthia M.; Gonzales, Karine; Chao, Yanjie; Pirzada, Zaid A. et al. (2011): CRISPR RNA maturation by trans-encoded small RNA and host factor RNase III. In: Nature 471 (7340), S. 602-607. DOI: 10.1038/nature09886.

Dietz, Marina S.; Haße, Daniel; Ferraris, Davide M.; Göhler, Antonia; Niemann, Hartmut H.; Heilemann, Mike (2013): Single-molecule photobleaching reveals increased MET receptor dimerization upon ligand binding in intact cells. In: $B M C$ biophysics 6 (1), S. 6. DOI: 10.1186/2046-1682-6-6.

Dietz, Marina S.; Heilemann, Mike (2019): Optical super-resolution microscopy unravels the molecular composition of functional protein complexes. In: Nanoscale 11 (39), S. 17981-17991. DOI: 10.1039/C9NR06364A.

Dikic, Ivan (2006): Endosomes. 1. Aufl. s.l.: Springer-Verlag (Molecular Biology Intelligence Unit). Online verfügbar unter http://gbv.eblib.com/patron/FullRecord.aspx?p=371759. 
Dorsch, Sandra; Klotz, Karl-Norbert; Engelhardt, Stefan; Lohse, Martin J.; Bünemann, Moritz (2009): Analysis of receptor oligomerization by FRAP microscopy. In: Nature methods 6 (3), S. 225-230. DOI: 10.1038/nmeth.1304.

Doyon, Jeffrey B.; Zeitler, Bryan; Cheng, Jackie; Cheng, Aaron T.; Cherone, Jennifer M.; Santiago, Yolanda et al. (2011): Rapid and efficient clathrin-mediated endocytosis revealed in genome-edited mammalian cells. In: Nature cell biology 13 (3), S. 331-337. DOI: $10.1038 / \mathrm{ncb} 2175$.

Durisic, Nela; Laparra-Cuervo, Lara; Sandoval-Álvarez, Angel; Borbely, Joseph Steven; Lakadamyali, Melike (2014): Single-molecule evaluation of fluorescent protein photoactivation efficiency using an in vivo nanotemplate. In: Nature methods 11 (2), S. 156-162. DOI: 10.1038/nmeth.2784.

Edelstein, Arthur D.; Tsuchida, Mark A.; Amodaj, Nenad; Pinkard, Henry; Vale, Ronald D.; Stuurman, Nico (2014): Advanced methods of microscope control using $\mu$ Manager software. In: J Biol Methods 1 (2), S. 10. DOI: 10.14440/jbm.2014.36.

Einstein, A. (1905): Zur Elektrodynamik bewegter Körper. In: Ann. Phys. 322 (10), S. 891-921. DOI: 10.1002/andp.19053221004.

Eklund, Alexandra S.; Ganji, Mahipal; Gavins, Georgina; Seitz, Oliver; Jungmann, Ralf (2020): Peptide-PAINT Super-Resolution Imaging Using Transient Coiled Coil Interactions. In: Nano letters 20 (9), S. 6732-6737. DOI: 10.1021/acs.nanolett.0c02620.

Ellenberg, J.; Lippincott-Schwartz, J.; Presley, J. F. (1998): Two-color green fluorescent protein time-lapse imaging. In: BioTechniques 25 (5), 838-42, 844-6. DOI: $10.2144 / 98255 \mathrm{bt} 01$.

Endesfelder, Ulrike; Malkusch, Sebastian; Flottmann, Benjamin; Mondry, Justine; Liguzinski, Piotr; Verveer, Peter J.; Heilemann, Mike (2011): Chemically induced photoswitching of fluorescent probes--a general concept for super-resolution microscopy. In: Molecules (Basel, Switzerland) 16 (4), S. 3106-3118. DOI: 10.3390/molecules16043106.

Endesfelder, Ulrike; Malkusch, Sebastian; Fricke, Franziska; Heilemann, Mike (2014): A simple method to estimate the average localization precision of a single-molecule localization microscopy experiment. In: Histochemistry and cell biology 141 (6), S. 629-638. DOI: 10.1007/s00418-014-1192-3.

Ferraris, Davide M.; Gherardi, Ermanno; Di, Ying; Heinz, Dirk W.; Niemann, Hartmut H. (2010): Ligand-mediated dimerization of the Met receptor tyrosine kinase by the bacterial invasion protein InlB. In: Journal of molecular biology 395 (3), S. 522-532. DOI: 10.1016/j.jmb.2009.10.074.

Finan, Kieran; Raulf, Anika; Heilemann, Mike (2015): A set of homo-oligomeric standards allows accurate protein counting. In: Angewandte Chemie (International ed. in English) 54 (41), S. 12049-12052. DOI: 10.1002/anie.201505664.

Franck, J.; Dymond, E. G. (1926): Elementary processes of photochemical reactions. In: Trans. Faraday Soc. 21 (February), S. 536. DOI: 10.1039/TF9262100536.

Freed, Daniel M.; Bessman, Nicholas J.; Kiyatkin, Anatoly; Salazar-Cavazos, Emanuel; Byrne, Patrick O.; Moore, Jason O. et al. (2017): EGFR Ligands Differentially Stabilize Receptor Dimers to Specify Signaling Kinetics. In: Cell 171 (3), 683-695.e18. DOI: 10.1016/j.cell.2017.09.017. 
Fricke, Franziska; Beaudouin, Joel; Eils, Roland; Heilemann, Mike (2015): One, two or three? Probing the stoichiometry of membrane proteins by single-molecule localization microscopy. In: Scientific reports 5, S. 14072. DOI: 10.1038/srep14072.

Fu, Changlin; Donovan, William P.; Shikapwashya-Hasser, Olga; Ye, Xudong; Cole, Robert H. (2014): Hot Fusion: an efficient method to clone multiple DNA fragments as well as inverted repeats without ligase. In: PloS one 9 (12), e115318. DOI: 10.1371/journal.pone. 0115318 .

Fueller, Julia; Herbst, Konrad; Meurer, Matthias; Gubicza, Krisztina; Kurtulmus, Bahtiyar; Knopf, Julia D. et al. (2020): CRISPR-Cas12a-assisted PCR tagging of mammalian genes. In: The Journal of cell biology 219 (6). DOI: $10.1083 /$ jcb.201910210.

Furge, K. A.; Zhang, Y. W.; Vande Woude, G. F. (2000): Met receptor tyrosine kinase. Enhanced signaling through adapter proteins. In: Oncogene 19 (49), S. 5582-5589. DOI: $10.1038 /$ sj.onc.1203859.

Fürstenberg, Alexandre; Heilemann, Mike (2013): Single-molecule localization microscopy-near-molecular spatial resolution in light microscopy with photoswitchable fluorophores. In: Physical chemistry chemical physics : PCCP 15 (36), S. 1491914930. DOI: 10.1039/C3CP52289J.

Garneau, Josiane E.; Dupuis, Marie-Ève; Villion, Manuela; Romero, Dennis A.; Barrangou, Rodolphe; Boyaval, Patrick et al. (2010): The CRISPR/Cas bacterial immune system cleaves bacteriophage and plasmid DNA. In: Nature 468 (7320), S. 6771. DOI: $10.1038 /$ nature09523.

Gasiunas, Giedrius; Barrangou, Rodolphe; Horvath, Philippe; Siksnys, Virginijus (2012): Cas9-crRNA ribonucleoprotein complex mediates specific DNA cleavage for adaptive immunity in bacteria. In: Proceedings of the National Academy of Sciences of the United States of America 109 (39), E2579-86. DOI: 10.1073/pnas.1208507109.

Ghaemmaghami, Sina; Huh, Won-Ki; Bower, Kiowa; Howson, Russell W.; Belle, Archana; Dephoure, Noah et al. (2003): Global analysis of protein expression in yeast. In: Nature 425 (6959), S. 737-741. DOI: 10.1038/nature02046.

Giannone, Gregory; Hosy, Eric; Levet, Florian; Constals, Audrey; Schulze, Katrin; Sobolevsky, Alexander I. et al. (2010): Dynamic Superresolution Imaging of Endogenous Proteins on Living Cells at Ultra-High Density. In: Biophysical journal 99 (4), S. 1303-1310. DOI: 10.1016/j.bpj.2010.06.005.

Gibson, Daniel G.; Young, Lei; Chuang, Ray-Yuan; Venter, J. Craig; Hutchison, Clyde A.; Smith, Hamilton O. (2009): Enzymatic assembly of DNA molecules up to several hundred kilobases. In: Nature methods 6 (5), S. 343-345. DOI: 10.1038/nmeth.1318.

Gibson, Toby J.; Seiler, Markus; Veitia, Reiner A. (2013): The transience of transient overexpression. In: Nature methods 10 (8), S. 715-721. DOI: 10.1038/nmeth.2534.

Girard, Tanya; Gaucher, Denis; El-Far, Mohamed; Breton, Gaëlle; Sékaly, RafickPierre (2014): CD80 and CD86 IgC domains are important for quaternary structure, receptor binding and co-signaling function. In: Immunology letters 161 (1), S. 65-75. DOI: 10.1016/j.imlet.2014.05.002.

Gordon, Matthew P.; Ha, Taekjip; Selvin, Paul R. (2004): Single-molecule highresolution imaging with photobleaching. In: Proceedings of the National Academy of 
Sciences of the United States of America 101 (17), S. 6462-6465. DOI: 10.1073/pnas.0401638101.

Greene, J. L.; Leytze, G. M.; Emswiler, J.; Peach, R.; Bajorath, J.; Cosand, W.; Linsley, P. S. (1996): Covalent dimerization of CD28/CTLA-4 and oligomerization of CD80/CD86 regulate T cell costimulatory interactions. In: The Journal of biological chemistry 271 (43), S. 26762-26771. DOI: 10.1074/jbc.271.43.26762.

Hale, Caryn R.; Zhao, Peng; Olson, Sara; Duff, Michael O.; Graveley, Brenton R.; Wells, Lance et al. (2009): RNA-guided RNA cleavage by a CRISPR RNA-Cas protein complex. In: Cell 139 (5), S. 945-956. DOI: 10.1016/j.cell.2009.07.040.

Hammond, D. E.; Urbé, S.; Vande Woude, G. F.; Clague, M. J. (2001): Downregulation of MET, the receptor for hepatocyte growth factor. In: Oncogene 20 (22), S. 2761-2770. DOI: 10.1038/sj.onc.1204475.

Hansen, Anders S.; Pustova, Iryna; Cattoglio, Claudia; Tjian, Robert; Darzacq, Xavier (2017): CTCF and cohesin regulate chromatin loop stability with distinct dynamics. In: eLife 6. DOI: 10.7554/eLife.25776.

Harwardt, Marie-Lena I. E.; Schröder, Mark S.; Li, Yunqing; Malkusch, Sebastian; Freund, Petra; Gupta, Shashi et al. (2020): Single-Molecule Super-Resolution Microscopy Reveals Heteromeric Complexes of MET and EGFR upon Ligand Activation. In: IJMS 21 (8). DOI: 10.3390/ijms21082803.

Harwardt, Marie-Lena I. E.; Young, Phoebe; Bleymüller, Willem M.; Meyer, Timo; Karathanasis, Christos; Niemann, Hartmut H. et al. (2017): Membrane dynamics of resting and internalin B-bound MET receptor tyrosine kinase studied by singlemolecule tracking. In: FEBS open bio 7 (9), S. 1422-1440. DOI: 10.1002/22115463.12285.

Heil, Christina S.; Rittner, Alexander; Goebel, Bjarne; Beyer, Daniel; Grininger, Martin (2018): Site-Specific Labelling of Multidomain Proteins by Amber Codon Suppression. In: Scientific reports 8 (1), S. 14864. DOI: 10.1038/s41598-018-33115-5.

Heilemann, Mike; van de Linde, Sebastian; Mukherjee, Anindita; Sauer, Markus (2009): Super-resolution imaging with small organic fluorophores. In: Angewandte Chemie (International ed. in English) 48 (37), S. 6903-6908. DOI:

10.1002/anie. 200902073.

Heilemann, Mike; van de Linde, Sebastian; Schüttpelz, Mark; Kasper, Robert; Seefeldt, Britta; Mukherjee, Anindita et al. (2008): Subdiffraction-resolution fluorescence imaging with conventional fluorescent probes. In: Angewandte Chemie (International ed. in English) 47 (33), S. 6172-6176. DOI: 10.1002/anie.200802376.

Heim, R.; Prasher, D. C.; Tsien, R. Y. (1994): Wavelength mutations and posttranslational autoxidation of green fluorescent protein. In: Proceedings of the National Academy of Sciences of the United States of America 91 (26), S. 12501-12504. DOI: 10.1073/pnas.91.26.12501.

Hess, Samuel T.; Huang, Shaohui; Heikal, Ahmed A.; Webb, Watt W. (2002): Biological and chemical applications of fluorescence correlation spectroscopy: a review. In: Biochemistry 41 (3), S. 697-705. DOI: 10.1021/bi0118512.

Higuchi, Osamu; Nakamura, Toshikazu (1991): Identification and change in the receptor for hepatocyte growth factor in rat liver after partial hepatectomy or induced 
hepatitis. In: Biochemical and Biophysical Research Communications 176 (2), S. 599607. DOI: $10.1016 / \mathrm{s} 0006-291 \times(05) 80226-8$.

Hoi, Hiofan; Shaner, Nathan C.; Davidson, Michael W.; Cairo, Christopher W.; Wang, Jiwu; Campbell, Robert E. (2010): A monomeric photoconvertible fluorescent protein for imaging of dynamic protein localization. In: Journal of molecular biology 401 (5), S. 776-791. DOI: 10.1016/j.jmb.2010.06.056.

Horisawa, Kenichi (2014): Specific and quantitative labeling of biomolecules using click chemistry. In: Frontiers in physiology 5, S. 457. DOI: 10.3389/fphys.2014.00457.

Huang, Bo; Babcock, Hazen; Zhuang, Xiaowei (2010): Breaking the diffraction barrier: super-resolution imaging of cells. In: Cell 143 (7), S. 1047-1058. DOI: 10.1016/j.cell.2010.12.002.

Huang, William Y. C.; Alvarez, Steven; Kondo, Yasushi; Lee, Young Kwang; Chung, Jean K.; Lam, Hiu Yue Monatrice et al. (2019): A molecular assembly phase transition and kinetic proofreading modulate Ras activation by SOS. In: Science (New York, N.Y.) 363 (6431), S. 1098-1103. DOI: 10.1126/science.aau5721.

Huang, Yongjian; Bharill, Shashank; Karandur, Deepti; Peterson, Sean M.; Marita, Morgan; Shi, Xiaojun et al. (2016): Molecular basis for multimerization in the activation of the epidermal growth factor receptor. In: eLife 5. DOI: 10.7554/eLife.14107.

Hubbard, S. R.; Till, J. H. (2000): Protein tyrosine kinase structure and function. In: Annual review of biochemistry 69, S. 373-398. DOI:

10.1146/annurev.biochem.69.1.373.

Hubbard, Stevan R.; Miller, W. Todd (2007): Receptor tyrosine kinases. Mechanisms of activation and signaling. In: Current opinion in cell biology 19 (2), S. 117-123. DOI: 10.1016/j.ceb.2007.02.010.

Hummer, Gerhard; Fricke, Franziska; Heilemann, Mike (2016): Model-independent counting of molecules in single-molecule localization microscopy. In: Molecular biology of the cell 27 (22), S. 3637-3644. DOI: 10.1091/mbc.E16-07-0525.

Hynes, Nancy E.; Schlange, Thomas (2006): Targeting ADAMS and ERBBs in lung cancer. In: Cancer cell 10 (1), S. 7-11. DOI: 10.1016/j.ccr.2006.06.012.

Ishino, Y.; Shinagawa, H.; Makino, K.; Amemura, M.; Nakata, A. (1987): Nucleotide sequence of the iap gene, responsible for alkaline phosphatase isozyme conversion in Escherichia coli, and identification of the gene product. In: Journal of bacteriology 169 (12), S. 5429-5433. DOI: 10.1128/jb.169.12.5429-5433.1987.

Jablonski, A. (1933): Efficiency of Anti-Stokes Fluorescence in Dyes. In: Nature 131 (3319), S. 839-840. DOI: 10.1038/131839b0.

James, John R.; Oliveira, Marta I.; Carmo, Alexandre M.; Iaboni, Andrea; Davis, Simon J. (2006): A rigorous experimental framework for detecting protein oligomerization using bioluminescence resonance energy transfer. In: Nature methods 3 (12), S. 10011006. DOI: $10.1038 /$ nmeth978.

Jayasinghe, Isuru; Clowsley, Alexander H.; Lin, Ruisheng; Lutz, Tobias; Harrison, Carl; Green, Ellen et al. (2018): True Molecular Scale Visualization of Variable Clustering Properties of Ryanodine Receptors. In: Cell reports 22 (2), S. 557-567. DOI: 10.1016/j.celrep.2017.12.045. 
Ji, Wei; Xu, Pingyong; Li, Zhengzheng; Lu, Jingze; Liu, Lin; Zhan, Yi et al. (2008): Functional stoichiometry of the unitary calcium-release-activated calcium channel. In: Proceedings of the National Academy of Sciences of the United States of America 105 (36), S. 13668-13673. DOI: 10.1073/pnas.0806499105.

Jinek, Martin; Chylinski, Krzysztof; Fonfara, Ines; Hauer, Michael; Doudna, Jennifer A.; Charpentier, Emmanuelle (2012): A programmable dual-RNA-guided DNA endonuclease in adaptive bacterial immunity. In: Science (New York, N.Y.) 337 (6096), S. 816-821. DOI: 10.1126/science.1225829.

Jungmann, Ralf; Avendaño, Maier S.; Dai, Mingjie; Woehrstein, Johannes B.; Agasti, Sarit S.; Feiger, Zachary et al. (2016): Quantitative super-resolution imaging with qPAINT. In: Nature methods 13 (5), S. 439-442. DOI: 10.1038/nmeth.3804.

Jungmann, Ralf; Steinhauer, Christian; Scheible, Max; Kuzyk, Anton; Tinnefeld, Philip; Simmel, Friedrich C. (2010): Single-molecule kinetics and super-resolution microscopy by fluorescence imaging of transient binding on DNA origami. In: Nano letters 10 (11), S. 4756-4761. DOI: $10.1021 / \mathrm{nl} 103427 \mathrm{w}$.

Kaboord, Barbara; Perr, Maria (2008): Isolation of proteins and protein complexes by immunoprecipitation. In: Methods in molecular biology (Clifton, N.J.) 424, S. 349-364. DOI: 10.1007/978-1-60327-064-9_27.

Kalir, Shiraz; Alon, Uri (2004): Using a quantitative blueprint to reprogram the dynamics of the flagella gene network. In: Cell 117 (6), S. 713-720. DOI: 10.1016/j.cell.2004.05.010.

Karathanasis, Christos; Fricke, Franziska; Hummer, Gerhard; Heilemann, Mike (2017): Molecule Counts in Localization Microscopy with Organic Fluorophores. In: Chemphyschem : a European journal of chemical physics and physical chemistry 18 (8), S. 942-948. DOI: 10.1002/cphc.201601425.

Karathanasis, Christos; Medler, Juliane; Fricke, Franziska; Smith, Sonja; Malkusch, Sebastian; Widera, Darius et al. (2020): Single-molecule imaging reveals the oligomeric state of functional TNF $\alpha$-induced plasma membrane TNFR1 clusters in cells. In: Science signaling 13 (614). DOI: 10.1126/scisignal.aax5647.

Kasha, Michael (1950): Characterization of electronic transitions in complex molecules. In: Discuss. Faraday Soc. 9, S. 14. DOI: 10.1039/DF9500900014.

Khan, Abdullah O.; Simms, Victoria A.; Pike, Jeremy A.; Thomas, Steven G.; Morgan, Neil V. (2017): CRISPR-Cas9 Mediated Labelling Allows for Single Molecule Imaging and Resolution. In: Scientific reports 7 (1), S. 8450. DOI: 10.1038/s41598-017-08493$\mathrm{X}$.

Khan, Abdullah O.; White, Carl W.; Pike, Jeremy A.; Yule, Jack; Slater, Alexandre; Hill, Stephen J. et al. (2019): Optimised insert design for improved single-molecule imaging and quantification through CRISPR-Cas9 mediated knock-in. In: Scientific reports 9 (1), S. 14219. DOI: 10.1038/s41598-019-50733-9.

Kim, Hui K.; Song, Myungjae; Lee, Jinu; Menon, A. Vipin; Jung, Soobin; Kang, Young-Mook et al. (2017): In vivo high-throughput profiling of CRISPR-Cpf1 activity. In: Nature methods 14 (2), S. 153-159. DOI: 10.1038/nmeth.4104.

Kleinstiver, Benjamin P.; Tsai, Shengdar Q.; Prew, Michelle S.; Nguyen, Nhu T.; Welch, Moira M.; Lopez, Jose M. et al. (2016): Genome-wide specificities of CRISPR- 
Cas Cpf1 nucleases in human cells. In: Nature biotechnology 34 (8), S. 869-874. DOI: 10.1038/nbt.3620.

Koniev, Oleksandr; Wagner, Alain (2015): Developments and recent advancements in the field of endogenous amino acid selective bond forming reactions for bioconjugation. In: Chemical Society reviews 44 (15), S. 5495-5551. DOI: 10.1039/C5CS00048C.

Krause, Daniela S.; van Etten, Richard A. (2005): Tyrosine kinases as targets for cancer therapy. In: The New England journal of medicine 353 (2), S. 172-187. DOI: 10.1056/NEJMra044389.

Krüger, Carmen L.; Zeuner, Marie-Theres; Cottrell, Graeme S.; Widera, Darius; Heilemann, Mike (2017): Quantitative single-molecule imaging of TLR4 reveals ligand-specific receptor dimerization. In: Science signaling 10 (503). DOI: 10.1126/scisignal.aan1308.

Lakowicz, Joseph R. (2006): Principles of Fluorescence Spectroscopy. Boston, MA: Springer US.

Le Cong; Ran, F. Ann; Cox, David; Lin, Shuailiang; Barretto, Robert; Habib, Naomi et al. (2013): Multiplex genome engineering using CRISPR/Cas systems. In: Science (New York, N.Y.) 339 (6121), S. 819-823. DOI: 10.1126/science.1231143.

Leake, Mark C.; Chandler, Jennifer H.; Wadhams, George H.; Bai, Fan; Berry, Richard M.; Armitage, Judith P. (2006): Stoichiometry and turnover in single, functioning membrane protein complexes. In: Nature 443 (7109), S. 355-358. DOI: 10.1038 /nature05135.

Lee, Sang-Hyuk; Shin, Jae Yen; Lee, Antony; Bustamante, Carlos (2012): Counting single photoactivatable fluorescent molecules by photoactivated localization microscopy (PALM). In: Proceedings of the National Academy of Sciences of the United States of America 109 (43), S. 17436-17441. DOI: 10.1073/pnas.1215175109.

Lemmon, Mark A.; Schlessinger, Joseph (2010): Cell signaling by receptor tyrosine kinases. In: Cell 141 (7), S. 1117-1134. DOI: 10.1016/j.cell.2010.06.011.

Lewis, T. S.; Shapiro, P. S.; Ahn, N. G. (1998): Signal transduction through MAP kinase cascades. In: Advances in cancer research 74, S. 49-139. DOI: 10.1016/s0065$230 x(08) 60765-4$.

Lewis Carl, S. A.; Gillete-Ferguson, I.; Ferguson, D. G. (1993): An indirect immunofluorescence procedure for staining the same cryosection with two mouse monoclonal primary antibodies. In: The journal of histochemistry and cytochemistry : official journal of the Histochemistry Society 41 (8), S. 1273-1278. DOI: 10.1177/41.8.7687266.

Li, Pilong; Banjade, Sudeep; Cheng, Hui-Chun; Kim, Soyeon; Chen, Baoyu; Guo, Liang et al. (2012): Phase transitions in the assembly of multivalent signalling proteins. In: Nature 483 (7389), S. 336-340. DOI: 10.1038/nature10879.

Li, Wenjing; Dick, Adam; Lu, Fei; Zhang, Hui; Sun, Hong (2019): Induction of MET Receptor Tyrosine Kinase Down-regulation through Antibody-mediated Receptor Clustering. In: Scientific reports 9 (1), S. 1988. DOI: 10.1038/s41598-018-36963-3. Li, Yanhua; Zhang, Xia; Pan, Wei; Li, Na; Tang, Bo (2020): A Nongenetic ProximityInduced FRET Strategy Based on DNA Tetrahedron for Visualizing the Receptor Dimerization. In: Analytical chemistry. DOI: 10.1021/acs.analchem.0c02330. 
Liang, Samantha I.; van Lengerich, Bettina; Eichel, Kelsie; Cha, Minkwon; Patterson, David M.; Yoon, Tae-Young et al. (2018): Phosphorylated EGFR Dimers Are Not Sufficient to Activate Ras. In: Cell reports 22 (10), S. 2593-2600. DOI: 10.1016/j.celrep.2018.02.031.

Liesche, Clarissa; Grussmayer, Kristin S.; Ludwig, Michael; Wörz, Stefan; Rohr, Karl; Herten, Dirk-Peter et al. (2015): Automated Analysis of Single-Molecule Photobleaching Data by Statistical Modeling of Spot Populations. In: Biophysical journal 109 (11), S. 2352-2362. DOI: 10.1016/j.bpj.2015.10.035.

Lin, Steven; Staahl, Brett T.; Alla, Ravi K.; Doudna, Jennifer A. (2014): Enhanced homology-directed human genome engineering by controlled timing of CRISPR/Cas9 delivery. In: eLife 3, e04766. DOI: 10.7554/eLife.04766.

Linsley, P. S.; Nadler, S. G.; Bajorath, J.; Peach, R.; Leung, H. T.; Rogers, J. et al. (1995): Binding stoichiometry of the cytotoxic T lymphocyte-associated molecule-4 (CTLA-4). A disulfide-linked homodimer binds two CD86 molecules. In: The Journal of biological chemistry 270 (25), S. 15417-15424. DOI: 10.1074/jbc.270.25.15417.

Lisenbee, Cayle S.; Karnik, Sheetal K.; Trelease, Richard N. (2003): Overexpression and mislocalization of a tail-anchored GFP redefines the identity of peroxisomal ER. In: Traffic (Copenhagen, Denmark) 4 (7), S. 491-501. DOI: 10.1034/j.16000854.2003.00107.x.

Liu, Wei; Wu, Aiping; Pellegrini, Matteo; Wang, Xiaofan (2015): Integrative analysis of human protein, function and disease networks. In: Scientific reports 5, S. 14344. DOI: $10.1038 /$ srep14344.

Lokker, N. A.; Mark, M. R.; Luis, E. A.; Bennett, G. L.; Robbins, K. A.; Baker, J. B.; Godowski, P. J. (1992): Structure-function analysis of hepatocyte growth factor. Identification of variants that lack mitogenic activity yet retain high affinity receptor binding. In: The EMBO journal 11 (7), S. 2503-2510.

Lukyanov, Konstantin A.; Chudakov, Dmitry M.; Lukyanov, Sergey; Verkhusha, Vladislav V. (2005): Innovation: Photoactivatable fluorescent proteins. In: Nature reviews. Molecular cell biology 6 (11), S. 885-891. DOI: 10.1038/nrm1741.

Madshus, Inger Helene; Stang, Espen (2009): Internalization and intracellular sorting of the EGF receptor. A model for understanding the mechanisms of receptor trafficking. In: Journal of cell science 122 (Pt 19), S. 3433-3439. DOI: 10.1242/jcs.050260.

Makarova, Kira S.; Grishin, Nick V.; Shabalina, Svetlana A.; Wolf, Yuri I.; Koonin, Eugene V. (2006): A putative RNA-interference-based immune system in prokaryotes. Computational analysis of the predicted enzymatic machinery, functional analogies with eukaryotic RNAi, and hypothetical mechanisms of action. In: Biology direct 1, S. 7. DOI: $10.1186 / 1745-6150-1-7$.

Mali, Prashant; Yang, Luhan; Esvelt, Kevin M.; Aach, John; Guell, Marc; DiCarlo, James E. et al. (2013): RNA-guided human genome engineering via Cas9. In: Science (New York, N.Y.) 339 (6121), S. 823-826. DOI: 10.1126/science.1232033.

Manley, Suliana; Gillette, Jennifer M.; Patterson, George H.; Shroff, Hari; Hess, Harald F.; Betzig, Eric; Lippincott-Schwartz, Jennifer (2008): High-density mapping of singlemolecule trajectories with photoactivated localization microscopy. In: Nature methods 5 (2), S. 155-157. DOI: 10.1038/nmeth.1176. 
Manzo, Carlo; Garcia-Parajo, Maria F. (2015): A review of progress in single particle tracking: from methods to biophysical insights. In: Reports on progress in physics. Physical Society (Great Britain) 78 (12), S. 124601. DOI: 10.1088/00344885/78/12/124601.

Marion, Dominique (2013): An introduction to biological NMR spectroscopy. In: Molecular \& cellular proteomics : MCP 12 (11), S. 3006-3025. DOI: 10.1074/mcp.O113.030239.

Marraffini, Luciano A.; Sontheimer, Erik J. (2008): CRISPR interference limits horizontal gene transfer in staphylococci by targeting DNA. In: Science (New York, N.Y.) 322 (5909), S. 1843-1845. DOI: 10.1126/science.1165771.

Martin-Fernandez, M. L.; Tynan, C. J.; Webb, S. E. D. (2013): A 'pocket guide' to total internal reflection fluorescence. In: Journal of microscopy 252 (1), S. 16-22. DOI: 10.1111/jmi.12070.

Matsumoto, K.; Nakamura, T. (2001): Hepatocyte growth factor: renotropic role and potential therapeutics for renal diseases. In: Kidney international 59 (6), S. 2023-2038. DOI: 10.1046/j.1523-1755.2001.00717.x.

Mayer, Bruce J.; Yu, Ji (2018): Protein Clusters in Phosphotyrosine Signal Transduction. In: Journal of molecular biology 430 (22), S. 4547-4556. DOI: 10.1016/j.jmb.2018.05.040.

McKinney, Sean A.; Murphy, Christopher S.; Hazelwood, Kristin L.; Davidson, Michael W.; Looger, Loren L. (2009): A bright and photostable photoconvertible fluorescent protein. In: Nature methods 6 (2), S. 131-133. DOI: 10.1038/nmeth.1296.

Michalet, Xavier (2010): Mean square displacement analysis of single-particle trajectories with localization error: Brownian motion in an isotropic medium. In: Physical review. E, Statistical, nonlinear, and soft matter physics 82 (4 Pt 1), S. 41914. DOI: 10.1103/PhysRevE.82.041914.

Michalet, Xavier; Berglund, Andrew J. (2012): Optimal diffusion coefficient estimation in single-particle tracking. In: Physical review. E, Statistical, nonlinear, and soft matter physics 85 (6 Pt 1), S. 61916. DOI: 10.1103/PhysRevE.85.061916.

Miller, Christina C. (1924): The Stokes-Einstein law for diffusion in solution. In: Proc. R. Soc. Lond. A 106 (740), S. 724-749. DOI: 10.1098/rspa.1924.0100.

Möckl, Leonhard; Lamb, Don C.; Bräuchle, Christoph (2014): Super-resolved fluorescence microscopy: Nobel Prize in Chemistry 2014 for Eric Betzig, Stefan Hell, and William E. Moerner. In: Angewandte Chemie (International ed. in English) 53 (51), S. 13972-13977. DOI: 10.1002/anie.201410265.

Moen, Erick; Bannon, Dylan; Kudo, Takamasa; Graf, William; Covert, Markus; van Valen, David (2019): Deep learning for cellular image analysis. In: Nature methods 16 (12), S. 1233-1246. DOI: 10.1038/s41592-019-0403-1.

Moerner, W. E.; Shechtman, Yoav; Wang, Quan (2015): Single-molecule spectroscopy and imaging over the decades. In: Faraday discussions 184, S. 9-36. DOI: 10.1039/C5FD00149H.

Mojica, F. J.; Díez-Villaseñor, C.; Soria, E.; Juez, G. (2000): Biological significance of a family of regularly spaced repeats in the genomes of Archaea, Bacteria and mitochondria. In: Molecular microbiology 36 (1), S. 244-246. DOI: 10.1046/j.13652958.2000.01838.x. 
Mojica, Francisco J. M.; Díez-Villaseñor, César; García-Martínez, Jesús; Soria, Elena (2005): Intervening sequences of regularly spaced prokaryotic repeats derive from foreign genetic elements. In: Journal of molecular evolution 60 (2), S. 174-182. DOI: 10.1007/s00239-004-0046-3.

Moreno-Mateos, Miguel A.; Fernandez, Juan P.; Rouet, Romain; Vejnar, Charles E.; Lane, Maura A.; Mis, Emily et al. (2017): CRISPR-Cpf1 mediates efficient homologydirected repair and temperature-controlled genome editing. In: Nature communications 8 (1), S. 2024. DOI: 10.1038/s41467-017-01836-2.

Morrison, Deborah K. (2012): MAP kinase pathways. In: Cold Spring Harbor perspectives in biology 4 (11). DOI: 10.1101/cshperspect.a011254.

Mortensen, Kim I.; Churchman, L. Stirling; Spudich, James A.; Flyvbjerg, Henrik (2010): Optimized localization analysis for single-molecule tracking and superresolution microscopy. In: Nature methods 7 (5), S. 377-381. DOI:

10.1038/nmeth.1447.

Mutch, Sarah A.; Fujimoto, Bryant S.; Kuyper, Christopher L.; Kuo, Jason S.; Bajjalieh, Sandra M.; Chiu, Daniel T. (2007): Deconvolving single-molecule intensity distributions for quantitative microscopy measurements. In: Biophysical journal 92 (8), S. 2926-2943. DOI: 10.1529/biophysj.106.101428.

Muthuswamy, S. K.; Gilman, M.; Brugge, J. S. (1999): Controlled dimerization of ErbB receptors provides evidence for differential signaling by homo- and heterodimers. In: Molecular and cellular biology 19 (10), S. 6845-6857. DOI: 10.1128/mcb.19.10.6845.

Nakamura, T.; Mizuno, S.; Matsumoto, K.; Sawa, Y.; Matsuda, H. (2000): Myocardial protection from ischemia/reperfusion injury by endogenous and exogenous HGF. In: The Journal of clinical investigation 106 (12), S. 1511-1519. DOI: 10.1172/JCI10226.

Nan, Xiaolin; Collisson, Eric A.; Lewis, Sophia; Huang, Jing; Tamgüney, Tanja M.; Liphardt, Jan T. et al. (2013): Single-molecule superresolution imaging allows quantitative analysis of RAF multimer formation and signaling. In: Proceedings of the National Academy of Sciences of the United States of America 110 (46), S. 1851918524. DOI: $10.1073 /$ pnas.1318188110.

Nasu, Yusuke; Benke, Alexander; Arakawa, Satoko; Yoshida, Go J.; Kawamura, Genki; Manley, Suliana et al. (2016): In Situ Characterization of Bak Clusters Responsible for Cell Death Using Single Molecule Localization Microscopy. In: Scientific reports 6, S. 27505. DOI: 10.1038/srep27505.

Needham, Sarah R.; Roberts, Selene K.; Arkhipov, Anton; Mysore, Venkatesh P.; Tynan, Christopher J.; Zanetti-Domingues, Laura C. et al. (2016): EGFR oligomerization organizes kinase-active dimers into competent signalling platforms. In: Nature communications 7, S. 13307. DOI: 10.1038/ncomms 13307.

Olshina, Maya A.; Sharon, Michal (2016): Mass Spectrometry: A Technique of Many Faces. In: Quarterly reviews of biophysics 49. DOI: 10.1017/S0033583516000160.

Organ, Shawna Leslie; Tsao, Ming-Sound (2011): An overview of the c-MET signaling pathway. In: Therapeutic advances in medical oncology 3 (1 Suppl), S7-S19. DOI: $10.1177 / 1758834011422556$.

Ormö, M.; Cubitt, A. B.; Kallio, K.; Gross, L. A.; Tsien, R. Y.; Remington, S. J. (1996): Crystal structure of the Aequorea victoria green fluorescent protein. In: Science (New York, N.Y.) 273 (5280), S. 1392-1395. DOI: 10.1126/science.273.5280.1392. 
Ortega, Claudia; Abreu, Cecilia; Oppezzo, Pablo; Correa, Agustín (2019): Overview of High-Throughput Cloning Methods for the Post-genomic Era. In: Methods in molecular biology (Clifton, N.J.) 2025, S. 3-32. DOI: 10.1007/978-1-4939-9624-7_1.

Padilla-Parra, Sergi; Tramier, Marc (2012): FRET microscopy in the living cell: different approaches, strengths and weaknesses. In: BioEssays : news and reviews in molecular, cellular and developmental biology 34 (5), S. 369-376. DOI: 10.1002/bies.201100086.

Paez-Segala, Maria G.; Sun, Mei G.; Shtengel, Gleb; Viswanathan, Sarada; Baird, Michelle A.; Macklin, John J. et al. (2015): Fixation-resistant photoactivatable fluorescent proteins for CLEM. In: Nature methods 12 (3), 215-8, 4 p following 218. DOI: $10.1038 /$ nmeth.3225.

Patterson, George H.; Lippincott-Schwartz, Jennifer (2002): A photoactivatable GFP for selective photolabeling of proteins and cells. In: Science (New York, N.Y.) 297 (5588), S. 1873-1877. DOI: 10.1126/science.1074952.

Penna, Aubin; Demuro, Angelo; Yeromin, Andriy V.; Zhang, Shenyuan L.; Safrina, Olga; Parker, Ian; Cahalan, Michael D. (2008): The CRAC channel consists of a tetramer formed by Stim-induced dimerization of Orai dimers. In: Nature 456 (7218), S. 116-120. DOI: 10.1038/nature07338.

Pickar-Oliver, Adrian; Gersbach, Charles A. (2019): The next generation of CRISPRCas technologies and applications. In: Nature reviews. Molecular cell biology 20 (8), S. 490-507. DOI: 10.1038/s41580-019-0131-5.

Planck, Max (1901): Ueber das Gesetz der Energieverteilung im Normalspectrum. In: Ann. Phys. 309 (3), S. 553-563. DOI: 10.1002/andp.19013090310.

Pletneva, Nadya V.; Pletnev, Sergei; Pakhomov, Alexey A.; Chertkova, Rita V.; Martynov, Vladimir I.; Muslinkina, Liya et al. (2016): Crystal structure of the fluorescent protein from Dendronephthya sp. in both green and photoconverted red forms. In: Acta crystallographica. Section D, Structural biology 72 (Pt 8), S. 922-932. DOI: $10.1107 / \mathrm{S} 205979831601038 X$.

Ponzetto, C.; Bardelli, A.; Zhen, Z.; Maina, F.; dalla Zonca, P.; Giordano, S. et al. (1994): A multifunctional docking site mediates signaling and transformation by the hepatocyte growth factor/scatter factor receptor family. In: Cell 77 (2), S. 261-271. DOI: $10.1016 / 0092-8674(94) 90318-2$.

Pourcel, C.; Salvignol, G.; Vergnaud, G. (2005): CRISPR elements in Yersinia pestis acquire new repeats by preferential uptake of bacteriophage DNA, and provide additional tools for evolutionary studies. In: Microbiology (Reading, England) $151(\mathrm{Pt}$ 3), S. 653-663. DOI: 10.1099/mic.0.27437-0.

Prasher, D. C.; Eckenrode, V. K.; Ward, W. W.; Prendergast, F. G.; Cormier, M. J. (1992): Primary structure of the Aequorea victoria green-fluorescent protein. In: Gene 111 (2), S. 229-233. DOI: 10.1016/0378-1119(92)90691-h.

Puchner, Elias M.; Walter, Jessica M.; Kasper, Robert; Huang, Bo; Lim, Wendell A. (2013): Counting molecules in single organelles with superresolution microscopy allows tracking of the endosome maturation trajectory. In: Proceedings of the National Academy of Sciences of the United States of America 110 (40), S. 16015-16020. DOI: 10.1073/pnas.1309676110. 
Ran, F. Ann; Hsu, Patrick D.; Wright, Jason; Agarwala, Vineeta; Scott, David A.; Zhang, Feng (2013): Genome engineering using the CRISPR-Cas9 system. In: Nature protocols 8 (11), S. 2281-2308. DOI: 10.1038/nprot.2013.143.

Ratz, Michael; Testa, Ilaria; Hell, Stefan W.; Jakobs, Stefan (2015): CRISPR/Cas9mediated endogenous protein tagging for RESOLFT super-resolution microscopy of living human cells. In: Scientific reports 5, S. 9592. DOI: 10.1038/srep09592.

Rayan, Gamal; Guet, Jean-Erik; Taulier, Nicolas; Pincet, Frederic; Urbach, Wladimir (2010): Recent applications of fluorescence recovery after photobleaching (FRAP) to membrane bio-macromolecules. In: Sensors (Basel, Switzerland) 10 (6), S. 5927-5948. DOI: $10.3390 / \mathrm{s} 100605927$.

Rayleigh (1896): On the theory of optical images, with special reference to the microscope. In: The London, Edinburgh, and Dublin Philosophical Magazine and Journal of Science 42 (255), S. 167-195. DOI: 10.1080/14786449608620902.

Renner, Marianne; Wang, Lili; Levi, Sabine; Hennekinne, Laetitia; Triller, Antoine (2017): A Simple and Powerful Analysis of Lateral Subdiffusion Using Single Particle Tracking. In: Biophysical journal 113 (11), S. 2452-2463. DOI:

10.1016/j.bpj.2017.09.017.

Renz, Malte (2013): Fluorescence microscopy-a historical and technical perspective. In: Cytometry. Part A : the journal of the International Society for Analytical Cytology 83 (9), S. 767-779. DOI: 10.1002/cyto.a.22295.

Roepstorff, Kirstine; Grøvdal, Lene; Grandal, Michael; Lerdrup, Mads; van Deurs, Bo (2008): Endocytic downregulation of ErbB receptors. Mechanisms and relevance in cancer. In: Histochemistry and cell biology 129 (5), S. 563-578. DOI: 10.1007/s00418008-0401-3.

Rossier, Olivier; Octeau, Vivien; Sibarita, Jean-Baptiste; Leduc, Cécile; Tessier, Béatrice; Nair, Deepak et al. (2012): Integrins $\beta 1$ and $\beta 3$ exhibit distinct dynamic nanoscale organizations inside focal adhesions. In: Nature cell biology 14 (10), S. 1057-1067. DOI: $10.1038 / \mathrm{ncb} 2588$.

Rust, Michael J.; Bates, Mark; Zhuang, Xiaowei (2006): Sub-diffraction-limit imaging by stochastic optical reconstruction microscopy (STORM). In: Nature methods 3 (10), S. 793-795. DOI: 10.1038/nmeth929.

Sapranauskas, Rimantas; Gasiunas, Giedrius; Fremaux, Christophe; Barrangou, Rodolphe; Horvath, Philippe; Siksnys, Virginijus (2011): The Streptococcus thermophilus CRISPR/Cas system provides immunity in Escherichia coli. In: Nucleic acids research 39 (21), S. 9275-9282. DOI: 10.1093/nar/gkr606.

Sauer, Markus; Heilemann, Mike (2017): Single-Molecule Localization Microscopy in Eukaryotes. In: Chemical reviews 117 (11), S. 7478-7509. DOI: 10.1021/acs.chemrev.6b00667.

Schlessinger, Joseph (2000): Cell Signaling by Receptor Tyrosine Kinases. In: Cell 103 (2), S. 211-225. DOI: 10.1016/S0092-8674(00)00114-8.

Schlessinger, Joseph (2002): Ligand-Induced, Receptor-Mediated Dimerization and Activation of EGF Receptor. In: Cell 110 (6), S. 669-672. DOI: 10.1016/S00928674(02)00966-2. 
Schmidt, C.; Bladt, F.; Goedecke, S.; Brinkmann, V.; Zschiesche, W.; Sharpe, M. et al. (1995): Scatter factor/hepatocyte growth factor is essential for liver development. In: Nature 373 (6516), S. 699-702. DOI: 10.1038/373699a0.

Schnitzbauer, Joerg; Strauss, Maximilian T.; Schlichthaerle, Thomas; Schueder, Florian; Jungmann, Ralf (2017): Super-resolution microscopy with DNA-PAINT. In: Nature protocols 12 (6), S. 1198-1228. DOI: 10.1038/nprot.2017.024.

Sengupta, Prabuddha; Jovanovic-Talisman, Tijana; Skoko, Dunja; Renz, Malte; Veatch, Sarah L.; Lippincott-Schwartz, Jennifer (2011): Probing protein heterogeneity in the plasma membrane using PALM and pair correlation analysis. In: Nature methods 8 (11), S. 969-975. DOI: 10.1038/nmeth.1704.

Shaner, Nathan C.; Campbell, Robert E.; Steinbach, Paul A.; Giepmans, Ben N. G.; Palmer, Amy E.; Tsien, Roger Y. (2004): Improved monomeric red, orange and yellow fluorescent proteins derived from Discosoma sp. red fluorescent protein. In: Nature biotechnology 22 (12), S. 1567-1572. DOI: 10.1038/nbt1037.

Shaner, Nathan C.; Steinbach, Paul A.; Tsien, Roger Y. (2005): A guide to choosing fluorescent proteins. In: Nature methods 2 (12), S. 905-909. DOI: 10.1038/nmeth819.

Sharma, Sreenath V.; Bell, Daphne W.; Settleman, Jeffrey; Haber, Daniel A. (2007): Epidermal growth factor receptor mutations in lung cancer. In: Nature reviews. Cancer 7 (3), S. 169-181. DOI: 10.1038/nrc2088.

Sharonov, Alexey; Hochstrasser, Robin M. (2006): Wide-field subdiffraction imaging by accumulated binding of diffusing probes. In: Proceedings of the National Academy of Sciences of the United States of America 103 (50), S. 18911-18916. DOI: 10.1073/pnas.0609643104.

Shashkova, Sviatlana; Leake, Mark C. (2017): Single-molecule fluorescence microscopy review: shedding new light on old problems. In: Bioscience reports 37 (4). DOI: 10.1042/BSR20170031.

Shcherbakova, Daria M.; Verkhusha, Vladislav V. (2014): Chromophore chemistry of fluorescent proteins controlled by light. In: Current opinion in chemical biology 20, S. 60-68. DOI: 10.1016/j.cbpa.2014.04.010.

Sherman, Eilon; Barr, Valarie; Manley, Suliana; Patterson, George; Balagopalan, Lakshmi; Akpan, Itoro et al. (2011): Functional nanoscale organization of signaling molecules downstream of the T cell antigen receptor. In: Immunity 35 (5), S. 705-720. DOI: 10.1016/j.immuni.2011.10.004.

Shimomura, O.; Johnson, F. H.; Saiga, Y. (1962): Extraction, purification and properties of aequorin, a bioluminescent protein from the luminous hydromedusan, Aequorea. In: Journal of cellular and comparative physiology 59, S. 223-239. DOI: 10.1002/jcp.1030590302.

Shroff, Hari; White, Helen; Betzig, Eric (2013): Photoactivated Localization Microscopy (PALM) of adhesion complexes. In: Current protocols in cell biology Chapter 4, Unit4.21. DOI: 10.1002/0471143030.cb0421s58.

Slade, Kristin M.; Baker, Rachael; Chua, Michael; Thompson, Nancy L.; Pielak, Gary J. (2009): Effects of recombinant protein expression on green fluorescent protein diffusion in Escherichia coli. In: Biochemistry 48 (23), S. 5083-5089. DOI: 10.1021/bi9004107. 
Slavik, J. M.; Hutchcroft, J. E.; Bierer, B. E. (1999): CD28/CTLA-4 and CD80/CD86 families: signaling and function. In: Immunologic research 19 (1), S. 1-24. DOI: 10.1007/BF02786473.

St Croix, Claudette M.; Shand, Stuart H.; Watkins, Simon C. (2005): Confocal microscopy: comparisons, applications, and problems. In: BioTechniques 39 (6 Suppl), S2-5. DOI: 10.2144/000112089.

Stepanenko, Aleksei; Andreieva, Svitlana; Korets, Kateryna; Mykytenko, Dmytro; Huleyuk, Nataliya; Vassetzky, Yegor; Kavsan, Vadym (2015): Step-wise and punctuated genome evolution drive phenotype changes of tumor cells. In: Mutation Research/Fundamental and Molecular Mechanisms of Mutagenesis 771, S. 56-69. DOI: 10.1016/j.mrfmmm.2014.12.006.

Stepanenko, Olesya V.; Stepanenko, Olga V.; Kuznetsova, Irina M.; Verkhusha, Vladislav V.; Turoverov, Konstantin K. (2013): Beta-barrel scaffold of fluorescent proteins. Folding, stability and role in chromophore formation. In: International review of cell and molecular biology 302, S. 221-278. DOI: 10.1016/B978-0-12-4076990.00004-2.

Stokes, George Gabriel (1852): On the change of refrangibility of light. In: Phil. Trans. R. Soc. 142, S. 463-562. DOI: 10.1098/rstl.1852.0022.

Strack, Rita (2018): Deep learning advances super-resolution imaging. In: Nature methods 15 (6), S. 403. DOI: 10.1038/s41592-018-0028-9.

Strauss, Sebastian; Nickels, Philipp C.; Strauss, Maximilian T.; Jimenez Sabinina, Vilma; Ellenberg, Jan; Carter, Jeffrey D. et al. (2018): Modified aptamers enable quantitative sub-10-nm cellular DNA-PAINT imaging. In: Nature methods 15 (9), S. 685-688. DOI: 10.1038/s41592-018-0105-0.

Subach, Fedor V.; Patterson, George H.; Manley, Suliana; Gillette, Jennifer M.; Lippincott-Schwartz, Jennifer; Verkhusha, Vladislav V. (2009): Photoactivatable mCherry for high-resolution two-color fluorescence microscopy. In: Nature methods 6 (2), S. 153-159. DOI: 10.1038/nmeth.1298.

Subach, Fedor V.; Verkhusha, Vladislav V. (2012): Chromophore transformations in red fluorescent proteins. In: Chemical reviews 112 (7), S. 4308-4327. DOI: $10.1021 / \mathrm{cr} 2001965$.

Sugiyama, Yoshiko; Kawabata, Izumi; Sobue, Kenji; Okabe, Shigeo (2005): Determination of absolute protein numbers in single synapses by a GFP-based calibration technique. In: Nature methods 2 (9), S. 677-684. DOI: 10.1038/nmeth783.

Suzuki, Takeshi; Matsuzaki, Toshiyuki; Hagiwara, Haruo; Aoki, Takeo; Takata, Kuniaki (2007): Recent advances in fluorescent labeling techniques for fluorescence microscopy. In: Acta histochemica et cytochemica 40 (5), S. 131-137. DOI: 10.1267/ahc.07023.

Teramura, Yuji; Ichinose, Junya; Takagi, Hiroaki; Nishida, Kenji; Yanagida, Toshio; Sako, Yasushi (2006): Single-molecule analysis of epidermal growth factor binding on the surface of living cells. In: The EMBO journal 25 (18), S. 4215-4222. DOI: 10.1038/sj.emboj.7601308.

Thevathasan, Jervis Vermal; Kahnwald, Maurice; Cieśliński, Konstanty; Hoess, Philipp; Peneti, Sudheer Kumar; Reitberger, Manuel et al. (2019): Nuclear pores as versatile 
reference standards for quantitative superresolution microscopy. In: Nature methods 16 (10), S. 1045-1053. DOI: 10.1038/s41592-019-0574-9.

Tokunaga, Makio; Imamoto, Naoko; Sakata-Sogawa, Kumiko (2008): Highly inclined thin illumination enables clear single-molecule imaging in cells. In: Nature methods 5 (2), S. 159-161. DOI: 10.1038/nmeth1171.

Toseland, Christopher P. (2013): Fluorescent labeling and modification of proteins. In: Journal of chemical biology 6 (3), S. 85-95. DOI: 10.1007/s12154-013-0094-5.

Trusolino, Livio; Comoglio, Paolo M. (2002): Scatter-factor and semaphorin receptors: cell signalling for invasive growth. In: Nature reviews. Cancer 2 (4), S. 289-300. DOI: $10.1038 /$ nrc779.

Turkowyd, Bartosz; Balinovic, Alexander; Virant, David; Carnero, Haruko G. Gölz; Caldana, Fabienne; Endesfelder, Marc et al. (2017): A General Mechanism of Photoconversion of Green-to-Red Fluorescent Proteins Based on Blue and Infrared Light Reduces Phototoxicity in Live-Cell Single-Molecule Imaging. In: Angewandte Chemie (International ed. in English) 56 (38), S. 11634-11639. DOI: 10.1002/anie.201702870.

Uehara, Y.; Minowa, O.; Mori, C.; Shiota, K.; Kuno, J.; Noda, T.; Kitamura, N. (1995): Placental defect and embryonic lethality in mice lacking hepatocyte growth factor/scatter factor. In: Nature 373 (6516), S. 702-705. DOI: 10.1038/373702a0.

Ulbrich, Maximilian H.; Isacoff, Ehud Y. (2007): Subunit counting in membrane-bound proteins. In: Nature methods 4 (4), S. 319-321. DOI: 10.1038/nmeth1024.

Ullrich, A.; Schlessinger, J. (1990): Signal transduction by receptors with tyrosine kinase activity. In: Cell 61 (2), S. 203-212. DOI: 10.1016/0092-8674(90)90801-k.

van de Linde, Sebastian; Aufmkolk, Sarah; Franke, Christian; Holm, Thorge; Klein, Teresa; Löschberger, Anna et al. (2013): Investigating cellular structures at the nanoscale with organic fluorophores. In: Chemistry \& biology 20 (1), S. 8-18. DOI: 10.1016/j.chembiol.2012.11.004.

Wachter, Rebekka (2017): Photoconvertible Fluorescent Proteins and the Role of Dynamics in Protein Evolution. In: IJMS 18 (8), S. 1792. DOI: 10.3390/ijms18081792.

Wang, Siyuan; Moffitt, Jeffrey R.; Dempsey, Graham T.; Xie, X. Sunney; Zhuang, Xiaowei (2014): Characterization and development of photoactivatable fluorescent proteins for single-molecule-based superresolution imaging. In: Proceedings of the National Academy of Sciences of the United States of America 111 (23), S. 8452-8457. DOI: $10.1073 /$ pnas.1406593111.

Ward, Colin W.; Lawrence, Michael C.; Streltsov, Victor A.; Adams, Timothy E.; McKern, Neil M. (2007): The insulin and EGF receptor structures: new insights into ligand-induced receptor activation. In: Trends in biochemical sciences 32 (3), S. 129137. DOI: $10.1016 /$ j.tibs.2007.01.001.

Weidner, K. M.; Di Cesare, S.; Sachs, M.; Brinkmann, V.; Behrens, J.; Birchmeier, W. (1996): Interaction between Gab1 and the c-Met receptor tyrosine kinase is responsible for epithelial morphogenesis. In: Nature 384 (6605), S. 173-176. DOI:

$10.1038 / 384173 \mathrm{a} 0$.

Wiedenmann, Jörg; Ivanchenko, Sergey; Oswald, Franz; Schmitt, Florian; Röcker, Carlheinz; Salih, Anya et al. (2004): EosFP, a fluorescent marker protein with UVinducible green-to-red fluorescence conversion. In: Proceedings of the National 
Academy of Sciences of the United States of America 101 (45), S. 15905-15910. DOI: 10.1073/pnas.0403668101.

Wolter, S.; Schättpelz, M.; Tscherepanow, M.; van de Linde, S.; Heilemann, M.; Sauer, M. (2010): Real-time computation of subdiffraction-resolution fluorescence images. In: Journal of microscopy 237 (1), S. 12-22. DOI: 10.1111/j.1365-2818.2009.03287.x.

Xie, Zhiping; Nair, Usha; Klionsky, Daniel J. (2008): Dissecting autophagosome formation: the missing pieces. In: Autophagy 4 (7), S. 920-922. DOI: 10.4161/auto.6692.

Yang, F.; Moss, L. G.; Phillips, G. N. (1996): The molecular structure of green fluorescent protein. In: Nature biotechnology 14 (10), S. 1246-1251. DOI: 10.1038/nbt1096-1246.

Yang, Yong; Wolfram, Joy; Fang, Xiaohong; Shen, Haifa; Ferrari, Mauro (2014): Polyarginine induces an antitumor immune response through binding to toll-like receptor 4. In: Small (Weinheim an der Bergstrasse, Germany) 10 (7), S. 1250-1254. DOI: $10.1002 / \mathrm{smll} .201302887$.

Zanacchi, Francesca Cella; Manzo, Carlo; Alvarez, Angel S.; Derr, Nathan D.; GarciaParajo, Maria F.; Lakadamyali, Melike (2017): A DNA origami platform for quantifying protein copy number in super-resolution. In: Nature methods 14 (8), S. 789-792. DOI: $10.1038 /$ nmeth.4342.

Zetsche, Bernd; Gootenberg, Jonathan S.; Abudayyeh, Omar O.; Slaymaker, Ian M.; Makarova, Kira S.; Essletzbichler, Patrick et al. (2015): Cpf1 is a single RNA-guided endonuclease of a class 2 CRISPR-Cas system. In: Cell 163 (3), S. 759-771. DOI: 10.1016/j.cell.2015.09.038.

Zhang, M. S.; Wu, L. J.; Xu, P. Y.; Liu, Y. F.; Xu, T. (2011): mEos2 Fluorescent Protein-Green Form.

Zhang, Mingshu; Chang, Hao; Zhang, Yongdeng; Yu, Junwei; Wu, Lijie; Ji, Wei et al. (2012): Rational design of true monomeric and bright photoactivatable fluorescent proteins. In: Nature methods 9 (7), S. 727-729. DOI: 10.1038/nmeth.2021.

Zhang, Xuewu; Schwartz, Jean-Claude D.; Almo, Steven C.; Nathenson, Stanley G. (2003): Crystal structure of the receptor-binding domain of human B7-2: insights into organization and signaling. In: Proceedings of the National Academy of Sciences of the United States of America 100 (5), S. 2586-2591. DOI: 10.1073/pnas.252771499.

Zhang, Yazhuo; Xia, Mengfang; Jin, Ke; Wang, Shufei; Wei, Hang; Fan, Chunmei et al. (2018): Function of the c-Met receptor tyrosine kinase in carcinogenesis and associated therapeutic opportunities. In: Molecular cancer 17 (1), S. 45. DOI: 10.1186/s12943018-0796-y.

Zhou, Xin X.; Lin, Michael Z. (2013): Photoswitchable fluorescent proteins: ten years of colorful chemistry and exciting applications. In: Current opinion in chemical biology 17 (4), S. 682-690. DOI: 10.1016/j.cbpa.2013.05.031. 


\section{Appendix}

A

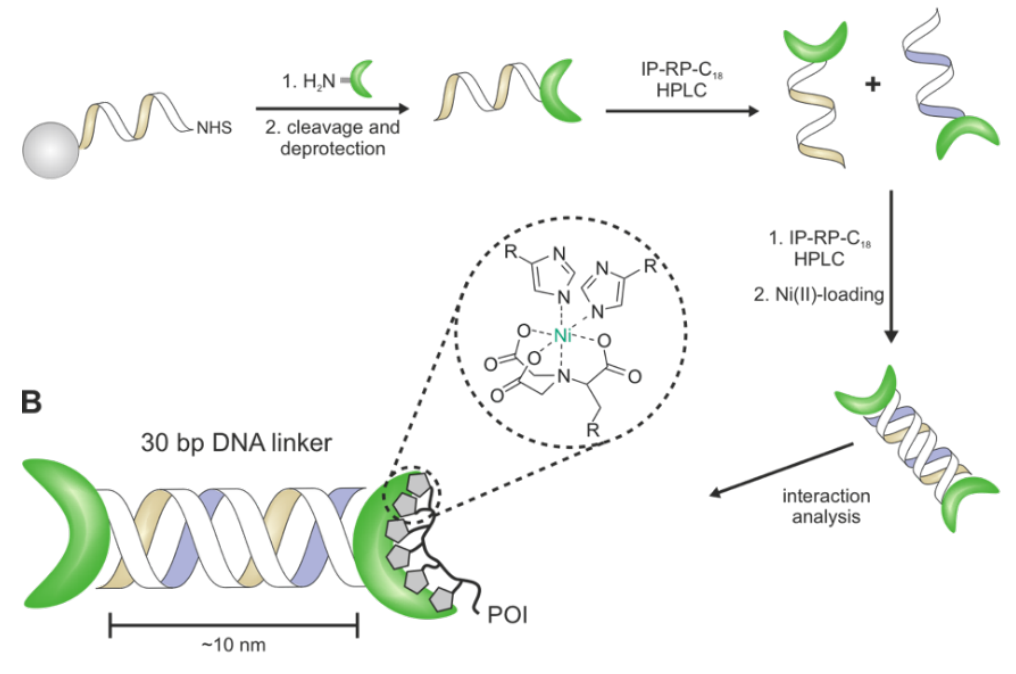

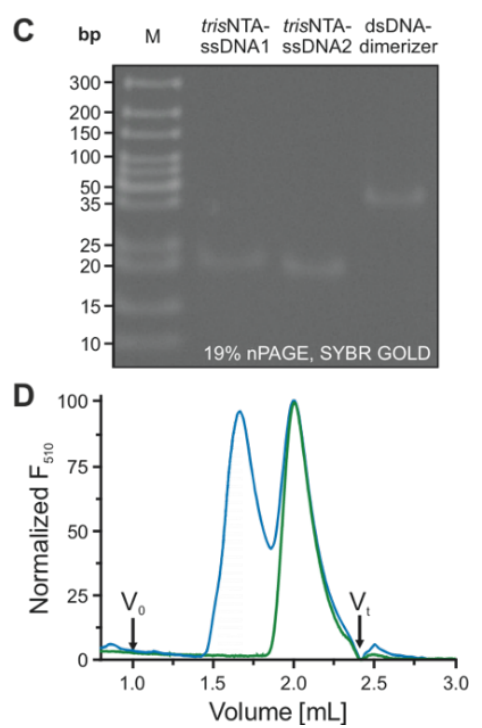

Figure App.4.1.2: Generation of the DNA-linker to crosslink two fluorescent his-tagged proteins. (A) Reaction scheme for the production of the 30 bp DNA linker. B) Schematic illustration of the product. C) Agarose gel of the tris-NTA attachment to the DNA-linker. D) Sizeexclusion profile to separate free mEos3.2 molecules (green) from the DNA-linked mEos3.2 molecules (blue). Adapted from Baldering et al (2019b). Copyright CC BY-NC-SA 3.0.
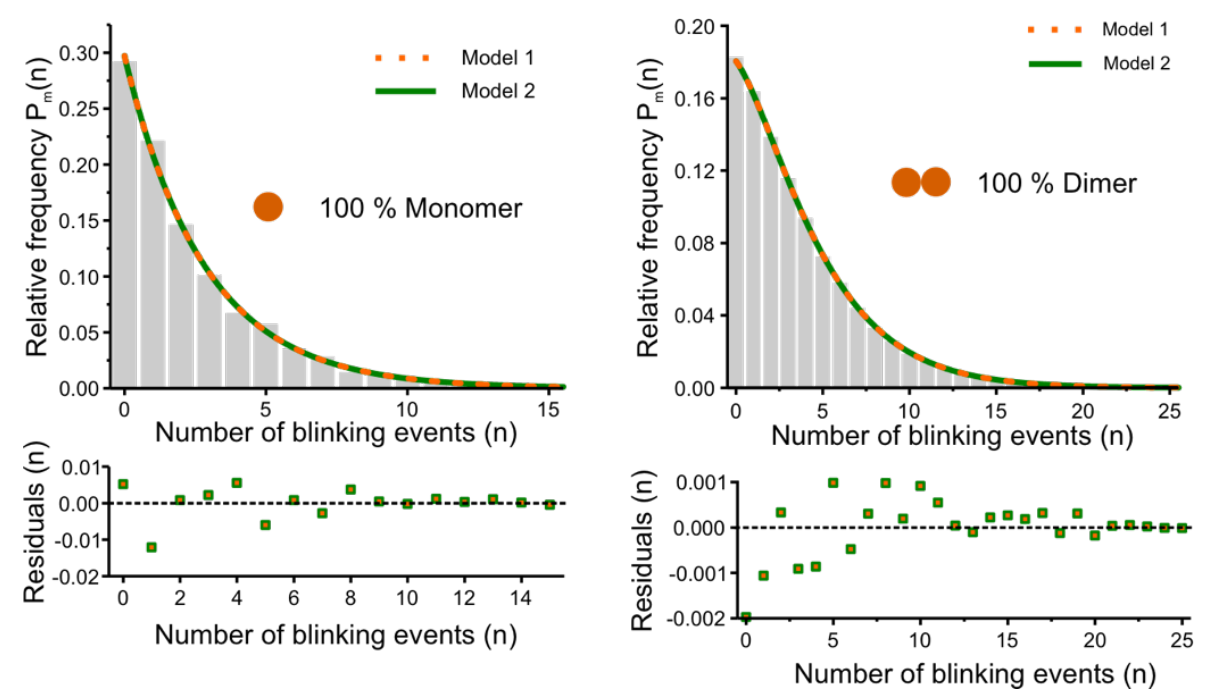

Figure App.4.2.1: Comparison of model 1 and 2 of simulated monomer and dimer data. (A)

Simulated qPALM data of monomers and dimers fitted by model 1 (orange dashed line) and 2 (green solid line) with $p=0.3$ and $d=0.57$ (top). The residuals of model 1 and 2 are shown in their respective color (bottom). Adapted from (Baldering et al 2019a). Copyright CC BY 4.0. 
A

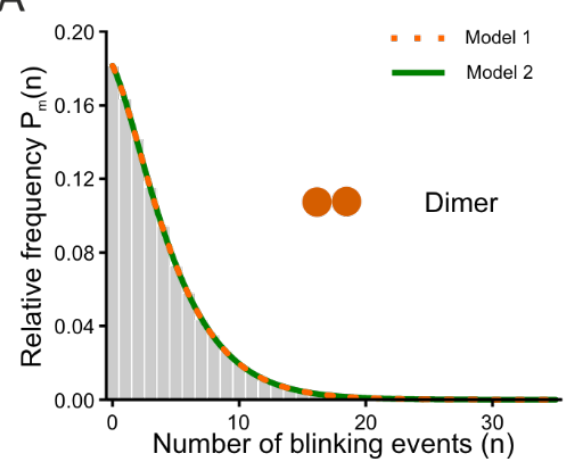

C

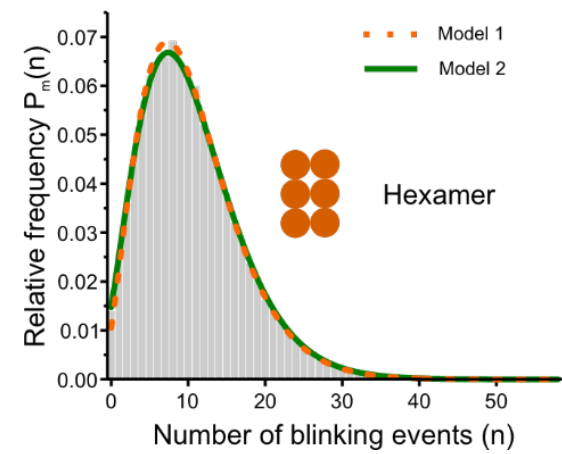

B

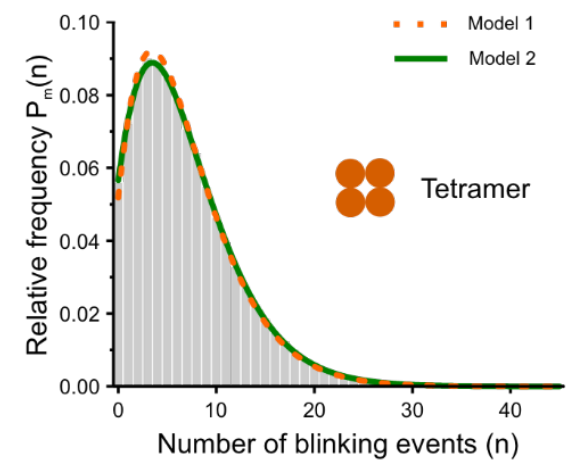

D

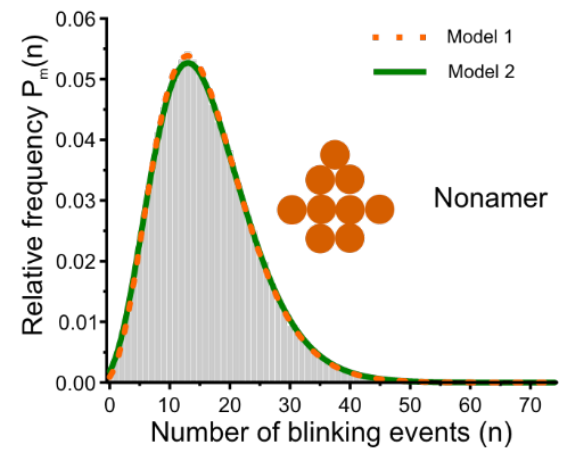

Figure App.4.2.2: Determining the q-value and detection efficiency from simulated data of oligomers. Simulated qPALM data of dimers (A), tetramers (B), hexamers (C), and nonamers (D) fitted by model 1 (orange dashed line) and 2 (green solid line) with $p=0.3$ and $q$ and $d$ were determined from the fit. For comparison, the $q$-value was translated into a detection efficiency by equation 19. (top) The residuals of model 1 and 2 are shown in their respective color (bottom). Adapted from (Baldering et al 2019a). Copyright CC BY 4.0. 


\section{List of abbreviations}

\begin{tabular}{|c|c|}
\hline AOTF & Acousto optic tunable filter \\
\hline $\mathrm{Bp}$ & Base pair \\
\hline CD86 & Cluster of differentiation 86 \\
\hline cDNA & complementary DNA \\
\hline CRISPR & Clustered regularly interspaced short palindromic repeats \\
\hline CTLA-4 & Cytotoxic T-lymphocyte-associated protein 4 \\
\hline crRNA & CRISPR RNA \\
\hline DMEM & Dulbecco's modified Eagle medium \\
\hline DNA & Deoxyribonucleic acid \\
\hline dSTORM & direct stochastic optical reconstruction microscopy \\
\hline DTT & dithiothreitol \\
\hline EGF & Epidermal growth factor \\
\hline EGFR & Epidermal growth factor receptor \\
\hline EMCCD & Electron multiplying charge coupled device \\
\hline FCS & Fluorescence correlation spectroscopy \\
\hline FRAP & Fluorescence recovery after photobleaching \\
\hline FRET & Förster resonance energy transfer \\
\hline FWHM & Full width at half maximum \\
\hline GFP & Green fluorescent protein \\
\hline Grb2 & Growth factor receptor-bound protein 2 \\
\hline HGF & Hepatocyte growth factor \\
\hline HILO & Highly inclined and laminated optical sheet \\
\hline HOMO & Highest occupied molecular orbital \\
\hline HRP & Horseradish peroxidase \\
\hline $\operatorname{IgG}$ & Immunoglobulin $\mathrm{G}$ \\
\hline InlB & Internalin B \\
\hline LAMA & Localization microscopy analyzer \\
\hline LUMO & Lowest unoccupied molecular orbital \\
\hline MAPK & Mitogen-activated protein kinase \\
\hline MEA & $\beta$-mercaptoethylamine \\
\hline MET & Mesenchymal-epithelial transition \\
\hline MSD & Mean square displacement \\
\hline NA & Numerical aperture \\
\hline
\end{tabular}




\begin{tabular}{|c|c|}
\hline $\mathrm{NeNa}$ & Nearest neighbor analysis \\
\hline NHS & N-hydroxysuccinimide \\
\hline PAINT & Points accumulation for imaging in nanoscale topography \\
\hline PALM & Photoactivated localization microscopy \\
\hline PBS & Phosphate buffered saline \\
\hline PCR & Polymerase chain reaction \\
\hline PDB & Protein data bank \\
\hline PI3K & Phosphatidylinositol 3-kinase \\
\hline PEG & Poly ethylene glycol \\
\hline PLL & Poly-L-lysine \\
\hline PSF & Point spread function \\
\hline PSI & Plexin-semaphorin-integrin \\
\hline qPAINT & $\begin{array}{l}\text { quantitative points accumulation for imaging in nanoscale } \\
\text { topography }\end{array}$ \\
\hline qPALM & quantitative photoactivated localization microscopy \\
\hline RNA & Ribonucleic acid \\
\hline RTK & Receptor tyrosine kinase \\
\hline SDS-PAGE & Sodium dodecyl sulfate polyacrylamide gel electrophoresis \\
\hline SEM & Standard error of the mean \\
\hline SMLM & Single-molecule localization microscopy \\
\hline SPT & Single-particle tracking \\
\hline STED & Stimulated emission depletion \\
\hline STORM & Stochastic optical reconstruction microscopy \\
\hline TIRF & Total internal reflection fluorescence \\
\hline TLR & Toll-like receptor \\
\hline TNFR & Tumor necrosis factor receptor \\
\hline uPAINT & $\begin{array}{l}\text { universal point accumulation for imaging in nanoscale } \\
\text { topography }\end{array}$ \\
\hline UV & ultraviolet \\
\hline VSVG & Vesicular stomatitis virus glycoprotein \\
\hline & wildtype \\
\hline
\end{tabular}




\section{List of figures}

Figure 1.1: Fluorescence microscopy tools to assess the dynamics and interactions of proteins.

Figure 1.2: Quantification in single-molecule localization microscopy.

Figure 1.3 Structure of receptor tyrosine kinases.

Figure 1.4: Overview of MET and EGFR signaling pathways.

Figure 2.1: Jablonski diagram.

Figure 2.2: Crystal structure of GFP.

Figure 2.3 Mechanism of photoactivation, -switching and -conversion of fluorescent proteins.

Figure 2.4: Crystal structure of mEos2.

Figure 2.5: Labeling schemes of target proteins with organic fluorophores.

Figure 2.6: Labeling schemes of target proteins with fluorescent proteins.

Figure 2.7: CRIPSR/Cas12a-assisted PCR tagging for genome labeling.

Figure 2.8: Illumination schemes of widefield, HILO, and TIRF.

Figure 2.9: Principle of single-molecule localization microscopy.

Figure 2.10: Overview of common SMLM techniques.

Figure 2.11: Counting of photoconvertible fluorescent proteins with qPALM.

Figure 2.12: Different diffusion types show distinct behaviors in the MSD plot.

Figure 3.1: Home-built microscope setup.

Figure 4.1.1: The workflow of qPALM.

Figure 4.1.2: Blinking properties of four fluorescent proteins determined by qPALM.

Figure 4.1.3: Blinking properties of $\mathrm{mEos} 4 \mathrm{~b}$ and $\mathrm{mEos} 3.2$.

Figure 4.1.4: Effect of reducing thiols on the blinking properties of mEos3.2 and mMaple3.

Figure 4.1.5: Blinking properties of mEos3.2A69T and Dendra2T69A.

Figure 4.1.6: Photon counts of different fluorescent proteins and with different buffer conditions.

Figure 4.1.7: Synthetic and genetic dimers as quantification rulers for the determination of the q-value in qPALM experiments.

Figure 4.1.8: CD86-mEos3.2 and CTLA-4-mEos3.2 serve as in cellulo calibration rulers for qPALM.

Figure 4.1.9: CD86-mEos4b and CTLA-4-mEos4b serve as in cellulo calibration rulers for qPALM.

Figure 4.1.10: Simulations of higher-ordered oligomers and mixtures of monomers and dimers. 
Figure 4.2.1: Model comparison for qSMLM analysis.

Figure 4.2.2: A comparison of model 1 in describing the blinking data of a simulated trimer.

Figure 4.2.3: A comparison of model 1 in describing the blinking data of higher-ordered oligomers.

Figure 4.2.4: Comparison of determined detection efficiencies from model 1 and 2 for different oligomeric states.

Figure 4.2.5: Deviations in the analysis of qPALM data due to undetected oligomers.

Figure 4.2.6: Accurate determination of multiple oligomers with complexity $\mathrm{m} \leq 2$.

Figure 4.2.7: Simulations of oligomers from single-molecule PALM data of mEos3.2.

Figure 4.3.1: Generation of PCR cassettes for CRISPR/Cas12a-assisted genome editing.

Figure 4.3.2: Fluorescence images of wildtype and CRISPR/Cas12a-modified HEK293T cells.

Figure 4.3.3: PCR of the edited part of the genome sequence in CRISPR/Cas12a-edited stable cell lines.

Figure 4.3.4: Western blots of MET-mEos4b and EGFR-mEos4b.

Figure 4.3.5: Western blots of receptor and MAPK phosphorylation to verify native signal transduction of MET-mEos4b and EGFR-mEos4b.

Figure 4.3.6: qPALM of MET-mEos4b in resting and HGF-stimulated HEK293T cells.

Figure 4.3.7: Quantitative PALM of InIB321-ATTO 647N-stimulated MET-mEos4b cells.

Figure 4.3.8: Single-particle-tracking of MET-mEos4b in resting and ligand-stimulated cells.

Figure 4.3.9: Comparison of the MSD analysis of sptPALM of MET-mEos4b and uPAINT measurements of InlB321-ATTO 647N.

Figure 4.3.10: Possible applications of PCR tagging in combination with SMLM.

Figure 4.4.1: Workflow of the QAFKA software.

Figure 4.4.2: QAFKA determines protein monomers and dimers in cells

Figure App.4.1.2: Generation of the DNA-linker to crosslink two fluorescent his-tagged proteins.

Figure App.4.2.1: Comparison of model 1 and 2 of simulated monomer and dimer data.

Figure App.4.2.2: Determining the q-value and detection efficiency from simulated data of oligomers. 


\section{List of tables}

Table 3.1: List of primers for cloning.

Table 3.2: List of generated plasmids.

Table 3.3: List of cell lines.

Table 3.4: List of primers for CRISPR/Cas12a-assisted PCR tagging.

Table 3.5: List of primers for genome analysis.

Table 4.1.1: $p$ - and $q$-values of various fluorescent proteins in vitro and in cellulo.

Table 4.2.1: Detection efficiencies obtained by model 1 and 2 for oligomers of different order.

Table 4.2.2: Accurate determination of mixtures of oligomers from qPALM data with model 2. 


\section{Acknowledgments}

During my doctoral thesis I received support from a large number of people, to whom I would like to thank very much. This circle of people includes in particular the whole working group around Professor Heilemann, my cooperation partners and additionally my family and friends.

First and foremost, I would like to thank Prof. Heilemann for his constant support and suggestions in the multitude of projects during my doctoral thesis. I am very pleased that I was given the opportunity to work on such an interesting topic in his research group. I am also very grateful that Prof. Heilemann gave me a lot of freedom for my own thoughts and supported them. I would especially like to thank him for the great time and experience during the conference of the Biophysical Society in San Diego and the very interesting conversations.

Furthermore, I am grateful to Prof. Gerhard Hummer for not only taking the time to review this dissertation, but also for helping me with the mathematically correct description of the new quantitative PALM model.

In addition, I would like to express my biggest thanks to Marina. You always had an open ear for any questions and were always able to give me important assistance. Especially, I would like to thank you for all the wonderful experiences and conversations, for example on the way home on our bikes. I appreciate you, among other qualities, for your care and reliability. I could always count on you, thank you for that!

I would also like to send a big thank you to the whole Heilemann working group for the great working atmosphere and general helpfulness of everyone. In my eyes, the atmosphere and way of dealing with people is unique and deserves the highest appreciation.

I would especially like to thank Christos, Sebastian, Marie-Lena, Petra and Mathilda who have contributed a lot to advance my projects. Special thanks to you Christos, for your great help with the measurements and evaluation of qPALM data. It was always fun with you and one could always rely on you. Sebastian, it was a pleasure to work with you in the team and it was a lot of fun to improve the theory of qPALM with you. You have contributed a lot to it. I appreciate not only your intelligence, but also your attitude to life. A very special thanks also goes to Marie-Lena, who has always helped me in my projects, 
especially with the analysis of single particle tracking data. I appreciate you very much for your whole personality and am grateful for all the nice conversations and memories. A big thanks also goes to Petra, who helped me a lot with providing cells and producing CRISPR/Cas12a cells. I really enjoy working with you in a team and I appreciate you for your work as well as for your character as a person in general. A huge thank you also goes to Mathilda, who introduced me to cloning techniques and thus also contributed to the successful completion of my doctoral thesis. Especially, I would like to thank you for your constant helpfulness and our interesting discussions. Thanks to Christoph for the funny lunchtime conversations and your incredibly friendly manner. I appreciate you as a person very much. Thanks also to Nina, for the nice conversations and your warm, friendly and helpful personality. A very big thanks also goes to Mark, who put a smile on my face every working day. It was a lot of fun to share my workspace with a good friend and it really made my work time more enjoyable. Besides work, I look back on wonderful evenings and swimming sessions. I really enjoy being friends with you and Marie-Lena. I would also like to thank Steffi for her help in organizational matters and Hans-Dieter for his help in working topics, as well as for great skiing days. Many thanks also to Marius, Caro, Ann-Christin, Yunqing, Peter, Jochen and former PhD students for their help and the wonderful time we spent together. I am also grateful to all my cooperation partners for supporting my projects and the nice atmosphere of cooperation.

Furthermore, I am grateful to be surrounded by my friends who make the time away from work great and give me valuable time to relax. The biggest thanks go to David, who always cheers me up and enriches my free time with his ideas. I thank you for your helpfulness and that I can always count on you. I am deeply grateful for our warm friendship and the extremely fun trips, activities and journeys together with Corinna and Carina.

Finally, I would like to say an indescribable thank you to my parents, my brother and my girlfriend. Dear Mom, dear Dad, I am incredibly grateful for everything you have made possible for me, for your support in every single step of my life and for the deep warmth and security you give me. I would also like to thank my brother Florian for believing in me and that we were always able to motivate each other to achieve our goals. An incredible thanks goes to Carina. Thank you so much for your support in giving me such a valuable and happy life with you, which motivates me incredibly to reach my goals and makes me look into the future with shining eyes. 


\section{Curriculum Vitae}

\section{Personal information}

$\begin{array}{ll}\text { Name: } & \text { Tim Niklas Baldering } \\ \text { Date of birth: } & \text { 18. April } 1992 \\ \text { Place of birth: } & \text { Bad Soden am Taunus }\end{array}$

\section{Education and research experience}

Since $04 / 2017$

$10 / 2014-03 / 2017$

$04 / 2016-06 / 2016$

$09 / 2011-08 / 2014$
$\mathrm{PhD}$ thesis at the Institute of Physical and Theoretical Chemistry, Johann Wolfgang Goethe-University, Frankfurt am Main, Germany

Master of Science in Biochemistry, Johann Wolfgang Goethe-University, Frankfurt am Main, Germany

Master thesis: NMR investigations on the cell-free expressed membrane-embedded protease OMA1 and the $\beta 1$-adrenergic receptor

Research Assistant in the group of Prof. Gerhard Wagner at the Havard Medical School, Boston, MA, USA

Bachelor of Science in Chemistry and Biochemistry, Ludwig-Maximilians-University, Munich, Germany

Bachelor Thesis: Characterization of three proteins (GLDH1A3, CSE1L and EPHX4) with respect to their localization and function on lipid droplets and evaluation of lipid droplet accumulation with three different fatty acids (LA, AA,PA) 
Abitur at the Albert-Einstein-Gymnasium, Schwalbach a. Ts. 


\section{Publications}

$12 / 2020$

Baldering, Tim N.; Karathanasis, Christos; Harwardt, Marie-Lena I.E.; Freund, Petra; Meurer, Matthias; Rahm, Johanna V.; Knop, Michael; Dietz, Marina S.; Heilemann, Mike (2020): CRISPR/Cas12a-mediated labeling of MET receptor enables quantitative single-molecule imaging of endogenous protein organization and dynamics. In: iScience, S. 101895. DOI: 10.1016/j.isci.2020.101895.

$09 / 2019$

Baldering, Tim N.; Bullerjahn, Jakob T.; Hummer, Gerhard; Heilemann, Mike; Malkusch, Sebastian (2019): Molecule counts in complex oligomers with singlemolecule localization microscopy. In: J. Phys. D: Appl. Phys. 52 (47), S. 474002. DOI: 10.1088/1361$6463 / \mathrm{ab} 3 \mathrm{~b} 65$.

$05 / 2019$ Baldering, Tim N.; Dietz, Marina S.; Gatterdam, Karl; Karathanasis, Christos; Wieneke, Ralph; Tampé, Robert; Heilemann, Mike (2019): Synthetic and genetic dimers as quantification ruler for single-molecule counting with PALM. In: Molecular biology of the cell 30 (12), S. 13691376. DOI: 10.1091/mbc.E18-10-0661.

$02 / 2019$ Böger, Carolin; Baldering, Tim N.; Krüger, Carmen L.; Harwardt, Marie-Lena; Mertinkus, Klara R.; Schröder, Mark S.; Karathanasis, Christos; Deussner-Helfmann, Nina S.; Barth, Hans-Dieter; Malkusch, Sebastian; Dietz, Marina S.; Hummer, Gerhard; Wohland, Thorsten \& Heilemann, Mike (2019) Quantification of membrane 
receptor complexes with single-molecule localization microscopy. In: SPIE 10884, 108840T. 